\title{
DESIGN AND PERFORMANCE OF CIRCULATION CONTROL FLAP GEOMETRIES
}

\author{
A Thesis \\ presented to \\ the Faculty of California Polytechnic State University, \\ San Luis Obispo

\begin{abstract}
In Partial Fulfillment
of the Requirements for the Degree

Master of Science in Aerospace Engineering
\end{abstract}

by

Rory M. Golden

March 2013 
(C) 2013

Rory M. Golden

ALL RIGHTS RESERVED 


\section{COMMITTEE MEMBERSHIP}

TITLE:

Design and Performance of Circulation Control

Flap Geometries

AUTHOR:

Rory M. Golden

DATE SUBMITTED:

March 2013

ADVISOR AND COMMITTEE CHAIR:

Dr. David Marshall

Associate Professor

Aerospace Engineering Dept.

COMMITTEE MEMBER:

Dr. Robert McDonald

Associate Professor

Aerospace Engineering Dept.

COMMITTEE MEMBER:

Dr. Kristina Jameson

Professor

Aerospace Engineering Dept.

COMMITTEE MEMBER:

Mr. Craig Hange

Aerospace Engineer

NASA Ames Research Center 


\section{ABSTRACT \\ DESIGN AND PERFORMANCE OF CIRCULATION CONTROL FLAP GEOMETRIES}

Rory M. Golden

With the pursuit of more advanced and environmentally-friendly technologies of today's society, the airline industry has been pushed further to investigate solutions that will reduce airport noise and congestion, cut down on emissions, and improve the overall performance of aircraft. These items directly influence airport size (runway length), flight patterns in the community surrounding the airport, cruise speed, and many other aircraft design considerations which are setting the requirements for next generation aircraft. Leading the research in this movement is NASA, which has set specific goals for the next generation regional airliners and has categorized the designs that meet the criteria as Cruise Efficient Short Takeoff and Land (CESTOL) aircraft.

With circulation control (CC) technology addressing most of the next generation requirements listed above, it has recently been gaining more interest, thus the basis of this research. CC is an active flow control method that uses a thin sheet of high momentum jet flow ejected over a curved trailing edge surface and in turn utilizes Coanda effect to increase the airfoil's circulation, augmenting lift, drag, and pitching moment. The technology has been around for more than 75 years, but is now gaining more momentum for further development due to its significant payoffs in both performance and system complexity.

The goal of this research was to explore the design of the CC flap shape and how it influences the local flow field of the system, in attempt to improve the performance of existing CC flap configurations and provide insight into the aerodynamic characteristics of the geometric parameters that make up the CC flap. Multiple dual radius flaps and alternative flap geometry, prescribed radius, flaps were developed by varying specific flap parameters from a baseline dual radius flap configuration that had been previously 
developed and researched. The aerodynamics of the various flap geometries were analyzed at three different flight conditions using two-dimensional CFD. The flight conditions examined include two low airspeed cases with blown flaps at $60^{\circ}$ and $90^{\circ}$ of deflection, and a transonic cruise case with no blowing and $0^{\circ}$ of flap deflection.

Results showed that the shorter flaps of both flap configurations augmented greater lift for the low airspeed cases, with the dual radius flaps producing more lift than the corresponding length prescribed radius. The large lift generation of these flaps was accompanied by significant drag and negative pitching moments. The incremental lift per drag and moment produced was best achieved by the longer flap lengths, with the prescribed radius flaps out-performing each corresponding dual radius. Longer flap configurations also upheld the better cruise performance with the least amount of low airspeed flow, drag, and required angle of attack for a given cruise lift coefficient. The prescribed radius flaps also presented a favorable trait of keeping a more continuous skin friction distribution over the flap when the flaps were deflected, where all dual radius configurations experienced a distinct fluctuation at the location where the surface curvature changes between its two radii. The prescribed radius flaps displayed a similar behavior when the flaps were not deflected, during the cruise conditions analyzed.

Performance trends for the different flap configurations, at all three flight conditions, are presented at the end of each respective section to provide guidance into the design of CC geometry. The results of the presented research show promise in modifying geometric surface parameters to yield improved aerodynamics and performance. 


\section{ACKNOWLEDGMENTS}

I would like to extend my first and foremost thanks to Dr. David Marshall for your persistent challenges, never letting me settle for anything less than excellent. Your curious imagination always kept me always thinking fresh and out of the box. Thank you for the opportunity to work for you on the AMELIA NASA grant and the invaluable experience that has provided me. Next, I'd like to thank Dr. Rob McDonald for your humor and ever-encouraging support. You've always provided plenty of wit to your lectures, while pushing me to award-winning efforts. Dr. Tina Jameson, you've always been a great example of the young professional that I strove to be. With your steady support, encouragement, and hard work ethic you kept me focused on my lofty goals. And I also have to thank you for showing me that you can always fit some fun in along the way. Bob Englar, thank you for inspiring me with your ever-lasting enthusiasm for the Circulation Control technology. Many thanks to Craig Hange for your backing of the AMELIA project along with accepting my last minute invite to serve on my thesis committee and defense.

I want to thank my parents for their support through my many years of college as I chased my dreams and enjoyed some life-changing experiences. Your encouragement to never let myself settle for anything but what I truly desired has paid off in the biggest ways. Thanks to my brother, Brad, for all the advice and time you've spent with me on the phone and in the water throughout school. It has kept my mind balanced, lifestyle healthy, and goals always within reach. I would also like to thank the rest of my extended family for your assistance and interest in all of my schoolings.

To my many friends that I've meet along the way, I cannot thank you enough for

the experiences we've been able to share. Whether it was in the classroom, taking breathers outside of the lab, or in the ocean, you've all shared in the many hardships of academia as well as taught me many life lessons. All of you will always have a friend in me, and I hope we can share some more good times in the future. 


\section{Table of Contents}

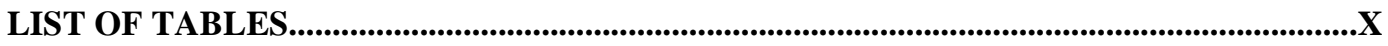

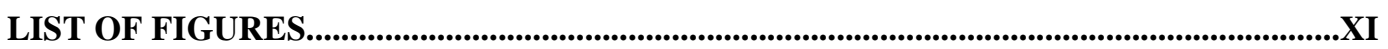

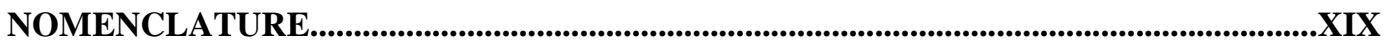

CHAPTER 1: INTRODUCTION ........................................................................................

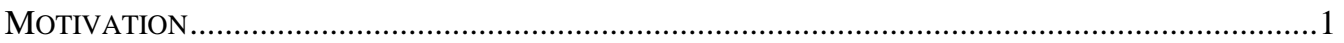

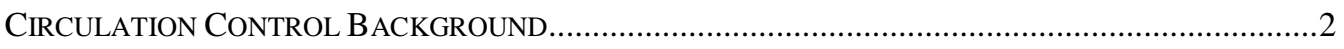

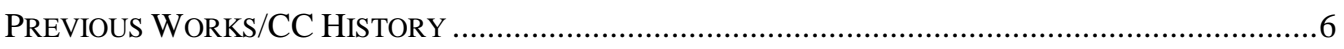

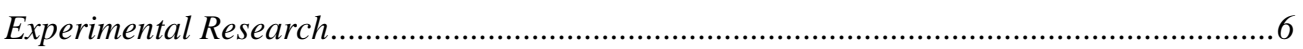

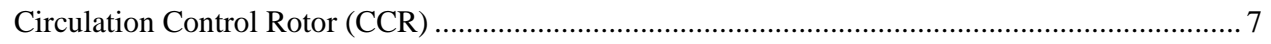

Circulation Control Airfoil Development ............................................................................... 8

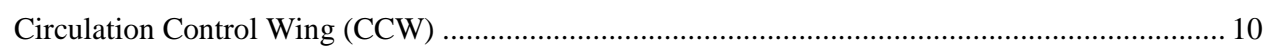

Advanced Circulation Control Airfoils........................................................................................ 11

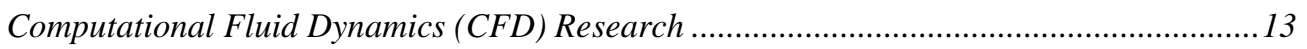

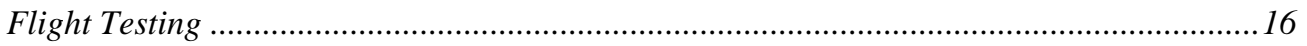

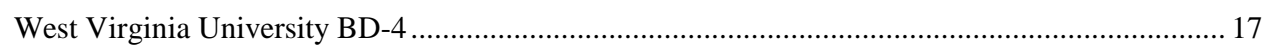

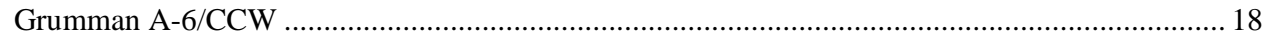

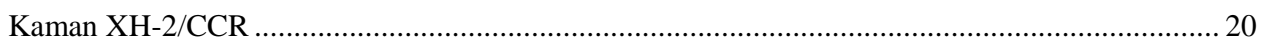

NASA Quiet Short-Haul Research Aircraft (QSRA) ………………………………………........ 21

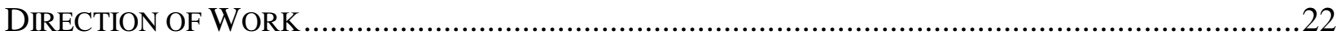

CHAPTER 2: PROBLEM STATEMENT .......................................................................24

CHAPTER 3: METHODOLOGY .................................................................................................28

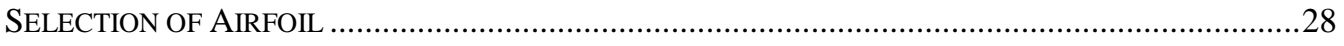

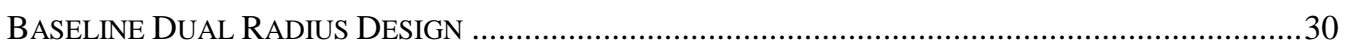

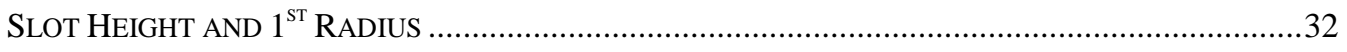

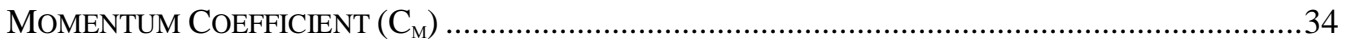


FLAP PLACEMENT/INTEGRATION

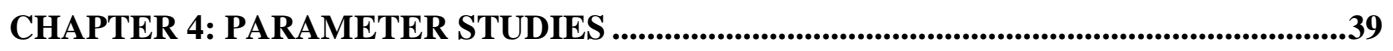

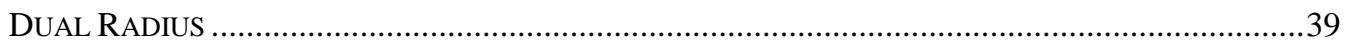

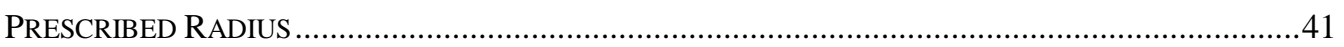

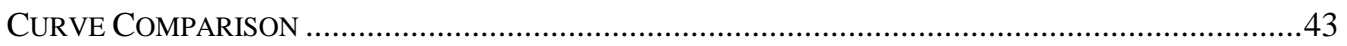

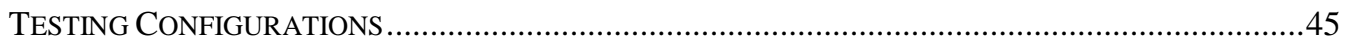

CHAPTER 5: COMPUTATIONAL ANALYSIS ARCHITECTURE ..................................48

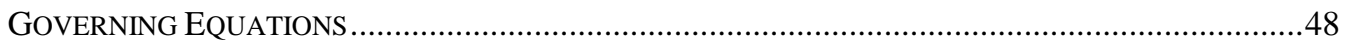

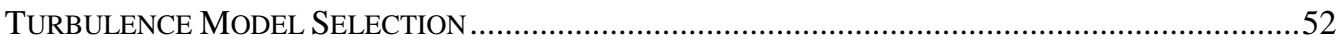

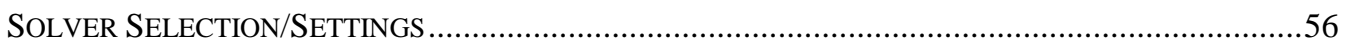

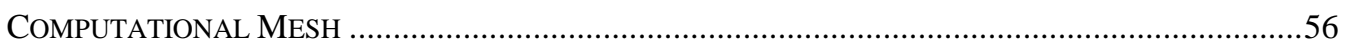

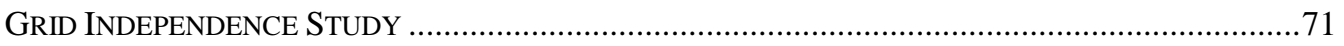

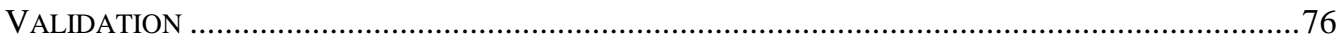

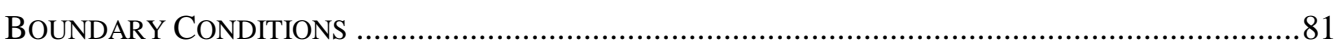

CHAPTER 6: RESULTS - PERFORMANCE ANALYSIS ...............................................86

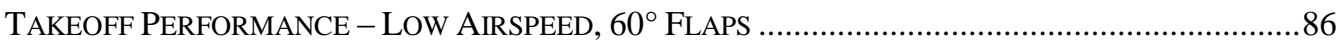

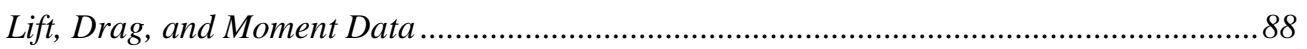

Pressure and Skin Friction Data .............................................................................. 94

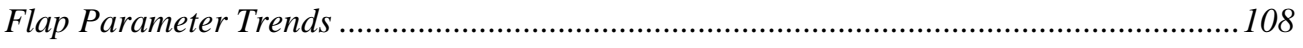

APPROACH/LANDING PERFORMANCE - LOW AIRSPEED, 90 FLAPS .................................... 115

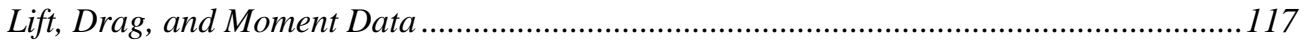

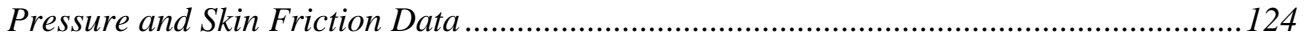

Flap Parameter Trends ................................................................................... 135

CRUISE PERFORMANCE - TrANSONIC AIRSPEED, $0^{\circ}$ FLAPS ........................................... 145

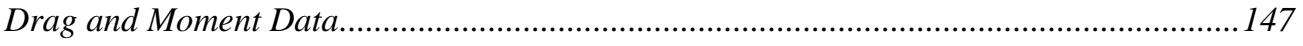

Pressure and Skin Friction Data ................................................................................. 152 


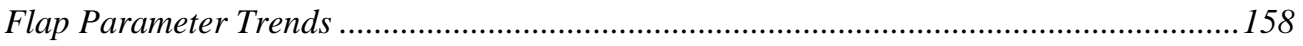

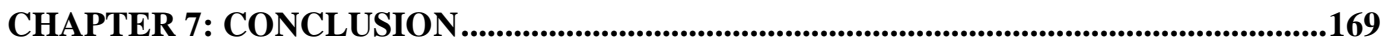

CHAPTER 8: FUTURE WORK .........................................................................................................171

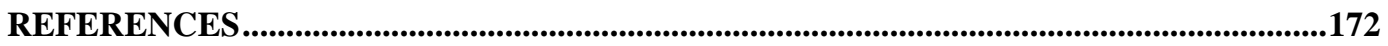




\section{List of Tables}

Table 2-1: NASA subsonic airliner performance goals for future aircraft ${ }^{19}, \ldots \ldots . .24$

Table 2-2: NASA goals for $\mathrm{N}+2100$ passenger airliner .................................. 25

Table 3-1: Baseline CC flap design parameters ........................................... 34

Table 3-2: Steps to place and integrate flap onto airfoil ................................ 37

Table 4-1: Flap configuration specifications ................................................. 46

Table 5-1: Fluent solver settings .............................................................. 56

Table 5-2: Mesh details and resulting solutions ........................................... 71

Table 5-3: Extrapolation terms, solutions, and errors .................................. 73

Table 5-4: Validation boundary conditions and settings ................................. 77

Table 5-5: Boundary conditions for all simulations ….................................. 85

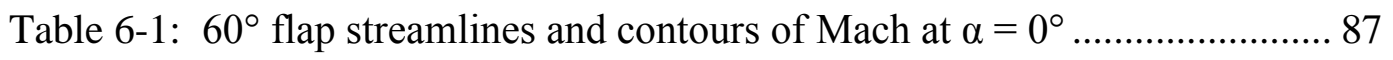

Table 6-2: $90^{\circ}$ flap streamlines and contours of Mach at $\alpha=0^{\circ} \ldots \ldots \ldots \ldots \ldots \ldots . . . . . . . .116$

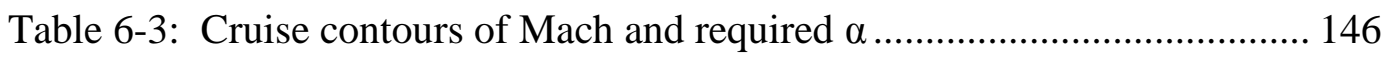

Table A-1: $60^{\circ}$ flap streamlines and contours of Mach at $\alpha=-5^{\circ}, 0^{\circ}, 3^{\circ} \ldots \ldots \ldots 187$

Table A-2: $90^{\circ}$ flap streamlines and contours of Mach at $\alpha=-5^{\circ}, 0^{\circ}, 3^{\circ} \ldots \ldots \ldots .190$ 


\section{List of Figures}

Figure 1-1: Cal Poly developed CESTOL aircraft......................................... 2

Figure 1-2: CC airfoil displaying the Coanda effect over trailing edge ${ }^{9} \ldots \ldots \ldots \ldots \ldots . . .4$

Figure 1-3: Streamlines during CC operation ..................................................... 4

Figure 1-4: NASA's X-Wing RSRA flight demonstrator (with main rotor) ${ }^{29} \ldots . . .8$

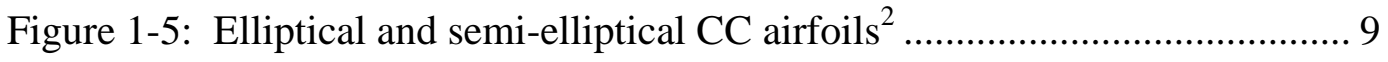

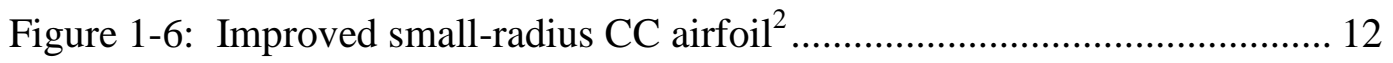

Figure 1-7: Supercritical airfoil with large leading edge and CC trailing edge ${ }^{2} .12$

Figure 1-8: Advanced dual radius CC airfoil with leading edge blowing ${ }^{2}$.......... 13

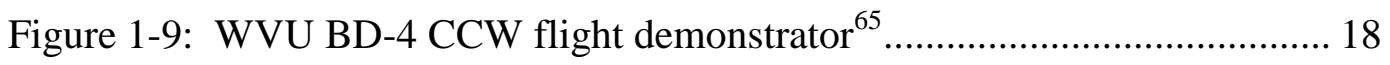

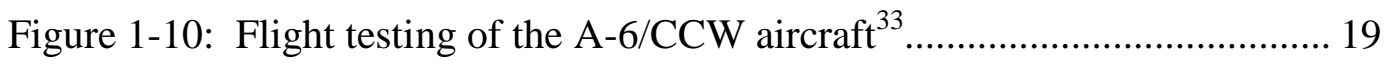

Figure 1-11: Kaman XH-2/CCR during hover flight test ${ }^{69}$.............................. 20

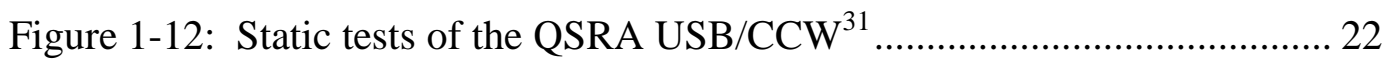

Figure 2-1: Three-view of AMELIA model .................................................... 25

Figure 2-2: Lifting efficiency for various blown high-lift systems ${ }^{32} \ldots \ldots \ldots \ldots \ldots \ldots \ldots . . .27$

Figure 3-1: NASA SC(2) - 0414 airfoil .......................................................... 29

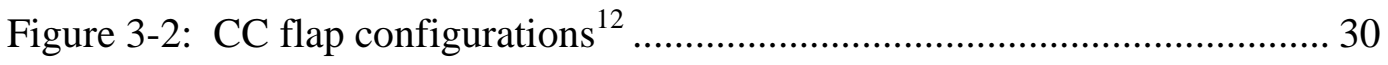

Figure 3-3: CC flap geometry investigated....................................................... 31

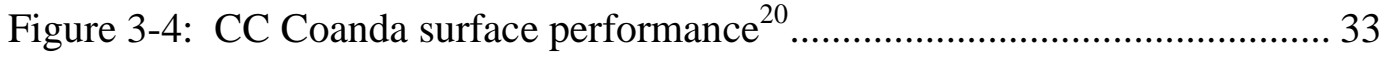


Figure 3-5: The two regions of circulation control as seen by experiment ${ }^{50} \ldots \ldots . .35$

Figure 3-6: Flap placement and integration ................................................. 36

Figure 3-7: Trailing edge slot modification ................................................ 38

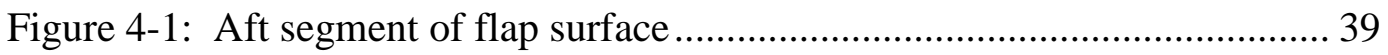

Figure 4-2: Previously studied CC dual radius flap configurations.................... 41

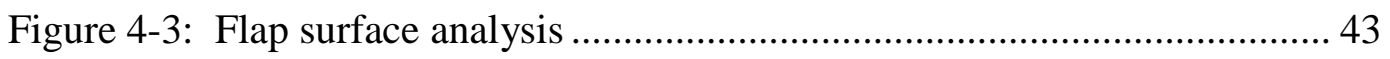

Figure 4-4: Curvature of flap surfaces ...................................................... 44

Figure 4-5: Examined flap configurations .................................................. 45

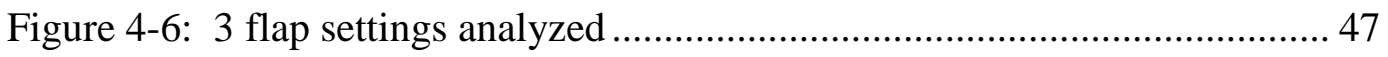

Figure 5-1: A standard velocity profile for a turbulent boundary layer ${ }^{89}$........... 52

Figure 5-2: Entire computational domain ............................................... 57

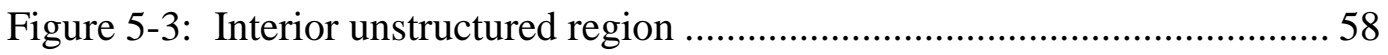

Figure 5-4: Different segments of structured grid .................................... 59

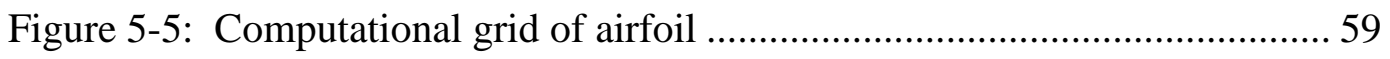

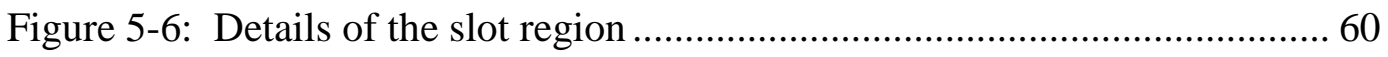

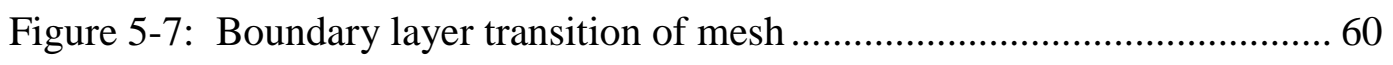

Figure 5-8: Contours of Mach overlaid mesh to shape structured regions .......... 61

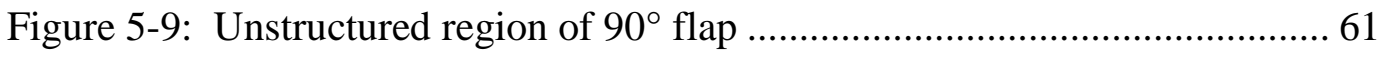

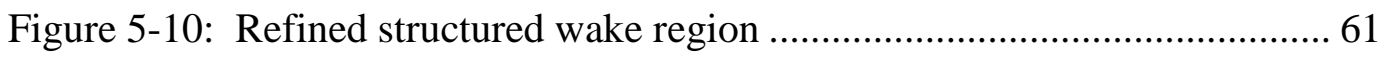


Figure 5-11: C-grid of cruise configuration simulation

Figure 5-12: Structured mesh around airfoil................................................ 70

Figure 5-13: Details of leading edge mesh .............................................. 70

Figure 5-14: Refined wake of trailing edge .............................................. 70

Figure 5-15: Solved and extrapolated lift coefficient results ........................... 74

Figure 5-16: Solved and extrapolated drag coefficient results ........................ 74

Figure 5-17: Solved and extrapolated moment coefficient results .................... 75

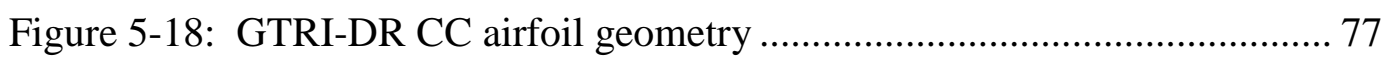

Figure 5-19: Lift coefficient given momentum coefficient comparison.............. 78

Figure 5-20: Airfoil pressure coefficient distribution comparison ..................... 79

Figure 5-21: Resulting $\mathrm{y}^{+}$values of validation case ..................................... 80

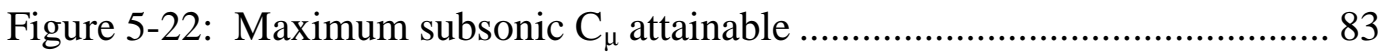

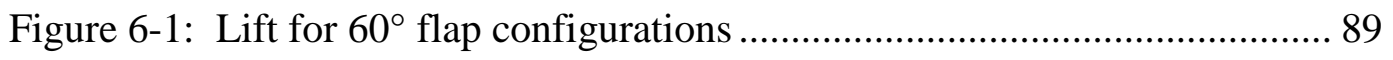

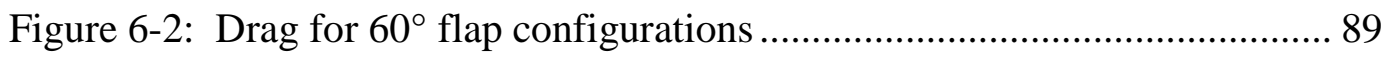

Figure 6-3: Drag given lift for $60^{\circ}$ flap configurations .................................. 91

Figure 6-4: Lift-to-drag ratios for $60^{\circ}$ flap configurations ............................. 92

Figure 6-5: Lift-to-drag ratios given lift for $60^{\circ}$ flap configurations .................. 93

Figure 6-6: Pitching moment for $60^{\circ}$ flap configurations................................ 93

Figure 6-7: Pressure distributions for $\alpha=0^{\circ}, 60^{\circ}$ flaps ................................. 95 
Figure 6-8: Leading edge suction peaks, $\alpha=0^{\circ}, 60^{\circ}$ flaps 96

Figure 6-9: Trailing edge suction peaks, $\alpha=0^{\circ}, 60^{\circ}$ flaps 97

Figure 6-10: Acceleration of slot flow on flap surface 98

Figure 6-11: Flap surface pressure distributions, $\alpha=0^{\circ}, 60^{\circ}$ flaps 99

Figure 6-12: Pressure distributions for $\alpha=-5^{\circ}, 60^{\circ}$ flaps 100

Figure 6-13: Pressure distributions for $\alpha=3^{\circ}, 60^{\circ}$ flaps 100

Figure 6-14: Leading edge suction peaks, $\alpha=3^{\circ}, 60^{\circ}$ flaps 102

Figure 6-15: Trailing edge suction peaks, $\alpha=3^{\circ}, 60^{\circ}$ flaps 102

Figure 6-16: Skin friction distributions for $\alpha=0^{\circ}, 60^{\circ}$ flaps 103

Figure 6-17: Flap surface skin friction distributions, $\alpha=0^{\circ}, 60^{\circ}$ flaps 104

Figure 6-18: Detailed skin friction at flap surface transition, $\alpha=0^{\circ}, 60^{\circ}$ flaps. 105

Figure 6-19: Flap curvature at transition from $r_{1}$ to $2^{\text {nd }}$ flap surface $\left(60^{\circ}\right)$ 106

Figure 6-20: $\mathrm{y}^{+}$values of the airfoil/flap configurations, $\alpha=0^{\circ}, 60^{\circ}$ flaps ....... 108

Figure 6-21: Lift-to-drag ratios for flap configurations given $\theta$ 109

Figure 6-22: Pitching moment for flap configurations given $\theta$ 110

Figure 6-23: Lift-to-drag ratios for flap configurations given $\mathrm{c}_{\mathrm{f}} / \mathrm{c}^{\prime}$ 111

Figure 6-24: Pitching moment for flap configurations given $\mathrm{c}_{\mathrm{f}} / \mathrm{c}^{\prime}$ 112

Figure 6-25: Lift-to-drag ratios for dual radius given $r_{2} / r_{1}$ 113

Figure 6-26: Pitching moment for dual radius given $r_{2} / r_{1}$ 113 
Figure 6-27: Lift-to-drag ratios for prescribed radius given $r_{2 \_a v g} / r_{1}$ 114

Figure 6-28: Pitching moment for prescribed radius given $r_{2}$ avg $/ r_{1}$ 114

Figure 6-29: Lift for $90^{\circ}$ flap configurations 118

Figure 6-30: Drag for $90^{\circ}$ flap configurations 118

Figure 6-31: Drag given lift for $90^{\circ}$ flap configurations. 120

Figure 6-32: Lift-to-drag ratios for $90^{\circ}$ flap configurations

Figure 6-33: Lift-to-drag ratios given lift for $90^{\circ}$ flap configurations 122

Figure 6-34: Pitching moment for $90^{\circ}$ flap configurations 123

Figure 6-35: Pressure distributions for $\alpha=0^{\circ}, 90^{\circ}$ flaps 124

Figure 6-36: Leading edge suction peaks, $\alpha=0^{\circ}, 90^{\circ}$ flaps 125

Figure 6-37: Trailing edge suction peaks, $\alpha=0^{\circ}, 90^{\circ}$ flaps 126

Figure 6-38: Flap surface pressure distributions, $\alpha=0^{\circ}, 90^{\circ}$ flaps 127

Figure 6-39: Pressure distributions for $\alpha=-5^{\circ}, 90^{\circ}$ flaps 128

Figure 6-40: Pressure distributions for $\alpha=3^{\circ}, 90^{\circ}$ flaps 128

Figure 6-41: Leading edge suction peaks, $\alpha=3^{\circ}, 90^{\circ}$ flaps 130

Figure 6-42: Trailing edge suction peaks, $\alpha=3^{\circ}, 90^{\circ}$ flaps

Figure 6-43: Skin friction distributions for $\alpha=0^{\circ}, 90^{\circ}$ flaps

Figure 6-44: Flap surface skin friction distributions, $\alpha=0^{\circ}, 90^{\circ}$ flaps 132

Figure 6-45: Detailed skin friction at flap surface transition, $\alpha=0^{\circ}, 90^{\circ}$ flaps. 133 
Figure 6-46: $\mathrm{y}^{+}$values of the airfoil/flap configurations, $\alpha=0^{\circ}, 90^{\circ}$ flaps ...... 134

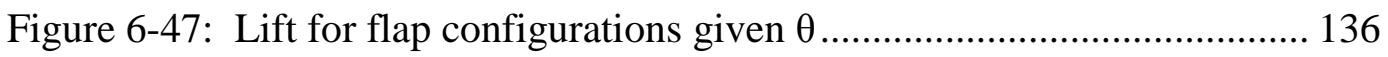

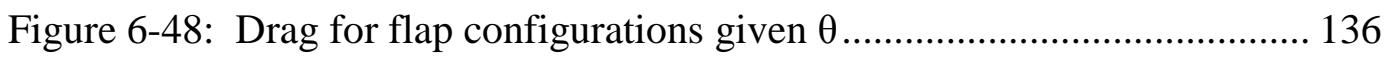

Figure 6-49: Pitching moment for flap configurations given $\theta$.................... 137

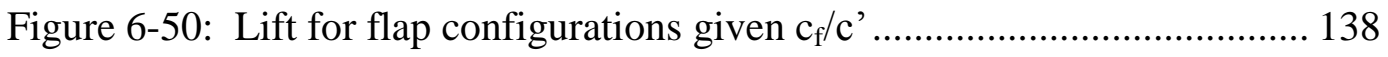

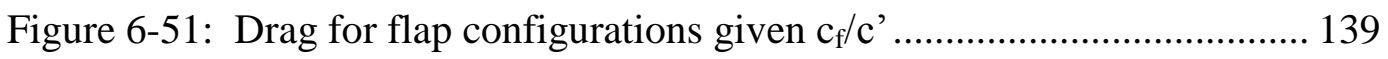

Figure 6-52: Pitching moment for flap configurations given $\mathrm{c}_{\mathrm{f}} / \mathrm{c}^{\prime}$.................. 139

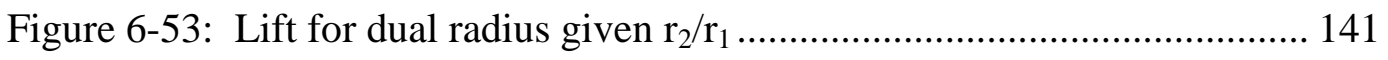

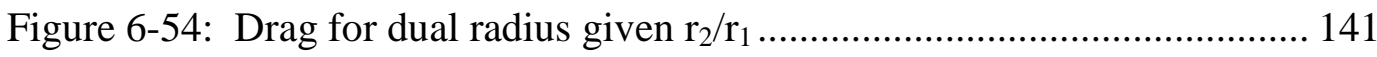

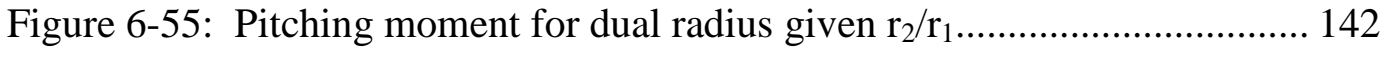

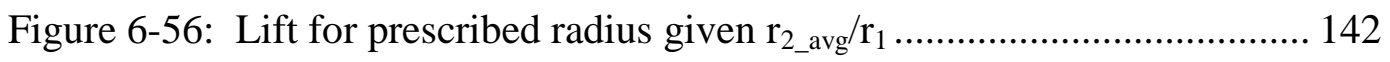

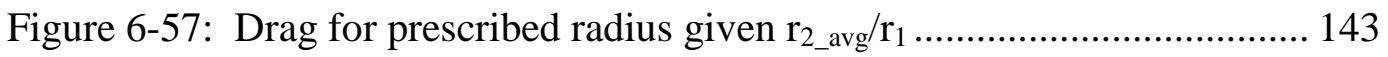

Figure 6-58: Pitching moment for prescribed radius given $r_{2 \_a v g} / r_{1} \ldots \ldots \ldots \ldots \ldots . . . . .143$

Figure 6-59: Required $\alpha$ for cruise given flap length for $0^{\circ}$ flap configurations 148

Figure 6-60: Drag given flap length for $0^{\circ}$ flap configurations ........................ 148

Figure 6-61: Drag and cruise $\alpha$ for $0^{\circ}$ flap configurations ............................ 150

Figure 6-62: Lift-to-drag ratios for $0^{\circ}$ flap configurations ............................ 150

Figure 6-63: Pitching moment given flap length for $0^{\circ}$ flap configurations...... 151

Figure 6-64: Trailing edge/flap surface pressure distribution, cruise $\alpha, 0^{\circ}$ flaps 153 
Figure 6-65: Airfoil trailing edge/flap surface curvature, cruise $\alpha, 0^{\circ}$ flaps...... 155

Figure 6-66: Flap surface skin friction distributions for cruise $\alpha, 0^{\circ}$ flaps ........ 156

Figure 6-67: $\mathrm{y}^{+}$values of the airfoil/flap configurations, cruise $\alpha, 0^{\circ}$ flaps $\ldots . . .157$

Figure 6-68: Lift-to-drag ratios for flap configurations given $\theta$.................... 159

Figure 6-69: Required cruise $\alpha$ for flap configurations given $\theta \ldots \ldots \ldots \ldots \ldots \ldots \ldots . . . . . . . . . .159$

Figure 6-70: Pitching moment for flap configurations given $\theta$.................... 160

Figure 6-71: Lift-to-drag ratios for flap configurations given $\mathrm{c}_{\mathrm{f}} / \mathrm{c}^{\prime} \ldots \ldots \ldots \ldots \ldots \ldots . . . . . .161$

Figure 6-72: Required cruise $\alpha$ for flap configurations given $c_{\mathrm{f}} / \mathrm{c}^{\prime}, \ldots \ldots \ldots \ldots \ldots . . . \ldots 162$

Figure 6-73: Pitching moment for flap configurations given $c_{f} / c^{\prime}{ }^{\prime} \ldots \ldots \ldots \ldots \ldots . . . . . . . .162$

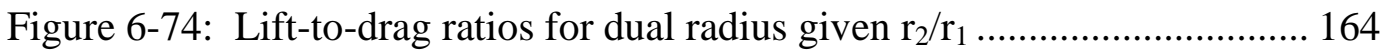

Figure 6-75: Required cruise $\alpha$ for dual radius given $r_{2} / r_{1} \ldots \ldots \ldots \ldots \ldots \ldots \ldots \ldots \ldots . . \ldots \ldots$

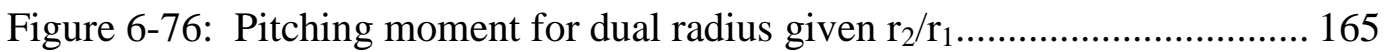

Figure 6-77: Lift-to-drag ratios for prescribed radius given $r_{2 \_a v g} / r_{1} \ldots \ldots \ldots \ldots \ldots . . . .166$

Figure 6-78: Required cruise $\alpha$ for prescribed radius given $r_{2 \_a v g} / r_{1} \ldots \ldots \ldots \ldots \ldots . \ldots 6$

Figure 6-79: Pitching moment for prescribed radius given $r_{2 \_a v g} / r_{1} \ldots \ldots \ldots \ldots \ldots \ldots . . . \ldots 7$

Figure A-9-1: Skin friction distributions for $\alpha=-5^{\circ}, 60^{\circ}$ flaps ...................... 188

Figure A-9-2: Skin friction distributions for $\alpha=3^{\circ}, 60^{\circ}$ flaps ....................... 188

Figure A-9-3: $\mathrm{y}^{+}$values of the airfoil/flap configurations, $\alpha=-5^{\circ}, 60^{\circ}$ flaps.... 189

Figure A-9-4: $\mathrm{y}^{+}$values of the airfoil/flap configurations, $\alpha=3^{\circ}, 60^{\circ}$ flaps ..... 189 
Figure A-9-5: Skin friction distributions for $\alpha=-5^{\circ}, 90^{\circ}$ flaps ...................... 191

Figure A-9-6: Skin friction distributions for $\alpha=3^{\circ}, 90^{\circ}$ flaps ........................ 191

Figure A-9-7: $\mathrm{y}^{+}$values of the airfoil/flap configurations, $\alpha=-5^{\circ}, 90^{\circ}$ flaps.... 192

Figure A-9-8: $y^{+}$values of the airfoil/flap configurations, $\alpha=3^{\circ}, 90^{\circ}$ flaps ..... 192 


\section{Nomenclature}

\section{English Symbols}

\begin{tabular}{|c|c|c|}
\hline Symbol & & Definition \\
\hline A & $=$ & area of slot exit $\left(\mathrm{m}^{2}\right)$ \\
\hline$A^{*}$ & $=$ & critical area of slot exit $\left(\mathrm{m}^{2}\right)$ \\
\hline $\mathrm{A}_{0}$ & $=$ & calibrated model constant \\
\hline$\Delta \mathrm{A}_{i}$ & $=$ & area of $i^{\text {th }}$ cell \\
\hline AR & $=$ & aspect ratio \\
\hline $\mathrm{A}_{\mathrm{s}}$ & $=$ & calibrated model constant \\
\hline $\mathrm{c}$ & $=$ & chord length (m) \\
\hline$c^{\prime}$ & $=$ & modified chord length (m) \\
\hline $\mathrm{c}_{\mathrm{f}}$ & $=$ & flap chord length (m) \\
\hline $\mathrm{C}^{+}$ & $=$ & dimensionless constant \\
\hline $\mathrm{C}_{1}$ & $=$ & calibrated model constant \\
\hline $\mathrm{C}_{1 \varepsilon}$ & $=$ & calibrated model constant \\
\hline $\mathrm{C}_{2}$ & $=$ & calibrated model constant \\
\hline $\mathrm{C}_{3 \varepsilon}$ & $=$ & calibrated model constant \\
\hline $\mathrm{C}_{\mathrm{d}}$ & $=$ & drag coefficient \\
\hline $\mathrm{C}_{\mathrm{f}}$ & $=$ & skin friction coefficient \\
\hline $\mathrm{C}_{1}$ & $=$ & 2-D lift coefficient \\
\hline $\mathrm{C}_{\mathrm{L}}$ & $=$ & 3-D lift coefficient \\
\hline $\mathrm{C}_{\mathrm{m},(\mathrm{c} / 4)}$ & $=$ & quarter-chord moment coefficient \\
\hline $\mathrm{C}_{\mu}$ & $=$ & momentum coefficient \\
\hline $\mathrm{C}_{\mu \mathrm{t}}$ & $=$ & calibrated model constant \\
\hline $\mathrm{C}_{\mathrm{p}}$ & $=$ & pressure coefficient \\
\hline $\mathrm{e}_{\infty}$ & $=$ & extrapolated relative error \\
\hline $\mathrm{e}_{\mathrm{a}}$ & $=$ & relative error \\
\hline$f$ & $=$ & elliptic relaxation factor \\
\hline $\mathrm{g}_{\mathrm{i}}$ & $=$ & gravity $\left(\mathrm{m} / \mathrm{s}^{2}\right)$ \\
\hline $\mathrm{h}$ & $=$ & average cell size \\
\hline $\mathrm{h}_{\text {slot }}$ & $=$ & trailing edge slot height $(\mathrm{m})$ \\
\hline $\mathrm{H}$ & $=$ & total enthalpy $(\mathrm{J})$ \\
\hline
\end{tabular}




\begin{tabular}{|c|c|c|}
\hline $\mathrm{k}$ & $=$ & turbulence kinetic energy \\
\hline $\mathrm{k}_{\mathrm{T}}$ & $=$ & thermal conductivity \\
\hline $\mathrm{L} / \mathrm{D}$ & $=$ & lift-to-drag ratio \\
\hline \multirow{2}{*}{$\begin{array}{l}\mathrm{L} /\left|\mathrm{M}_{(\mathrm{c} / 4)}\right| \\
\dot{m}\end{array}$} & $=$ & lift-to-quarter chord moment ratio \\
\hline & $=$ & 2-D mass flow rate $(\mathrm{kg} /(\mathrm{m} * \mathrm{~s}))$ \\
\hline M & $=$ & Mach number \\
\hline$n$ & $=$ & order of solution \\
\hline $\mathrm{N}$ & $=$ & generation of aircraft defined by NASA \\
\hline$N$ & $=$ & total number of cells per mesh \\
\hline $\mathrm{NO}_{\mathrm{x}}$ & $=$ & nitrogen dioxide \\
\hline $\mathrm{p}$ & $=$ & static pressure $(\mathrm{Pa})$ \\
\hline $\mathrm{P}$ & $=$ & pressure $(\mathrm{Pa})$ \\
\hline $\mathrm{P}_{\mathrm{b}}$ & $=$ & generation of $\mathrm{k}$ due to buoyancy \\
\hline $\mathrm{P}_{\mathrm{k}}$ & $=$ & generation of $\mathrm{k}$ due to mean velocity gradients \\
\hline $\operatorname{Pr}_{t}$ & $=$ & turbulent Prandtl number \\
\hline $\mathrm{q}$ & $=$ & dynamic pressure $(\mathrm{Pa})$ \\
\hline $\mathrm{r}$ & $=$ & radius $(\mathrm{m})$ \\
\hline $\mathrm{r}_{1}$ & $=$ & first radius of CC flap (m) \\
\hline $\mathrm{r}_{2}$ & $=$ & second radius of $\mathrm{CC}$ dual radius flap (m) \\
\hline $\mathrm{r}_{2 \_ \text {avg }}$ & $=$ & average radius of second prescribed radius flap ( $\mathrm{m}$ \\
\hline $\mathrm{r}_{i j}$ & $=$ & grid refinement factor \\
\hline $\mathrm{R}$ & $=$ & specific gas constant \\
\hline $\mathrm{R}_{\mathrm{ij}}$ & $=$ & Reynolds stress tensor \\
\hline$S$ & $=$ & modulus of mean rate-of-strain tensor \\
\hline $\mathrm{S}_{\varepsilon}$ & $=$ & user-defined source term \\
\hline$S_{i j}$ & $=$ & mean rate-of-strain tensor \\
\hline $\mathrm{S}_{\mathrm{k}}$ & $=$ & user-defined source term \\
\hline $\mathrm{T}$ & $=$ & temperature $(\mathrm{K})$ \\
\hline $\mathrm{u}$ & $=$ & Cartesian velocity component $(\mathrm{m} / \mathrm{s})$ \\
\hline $\mathrm{u}^{+}$ & $=$ & dimensionless velocity component \\
\hline $\mathrm{u}_{\tau}$ & $=$ & friction velocity $(\mathrm{m} / \mathrm{s})$ \\
\hline $\mathrm{U}^{*}$ & $=$ & model term \\
\hline$v^{2}$ & $=$ & velocity scale \\
\hline $\mathrm{V}$ & $=$ & velocity $(\mathrm{m} / \mathrm{s})$ \\
\hline $\mathrm{x}$ & & chord-wise direction \\
\hline$x$ & & Cartesian coordinate \\
\hline
\end{tabular}




$$
\begin{array}{ll}
\mathrm{y} & =\text { distance to the wall/boundary }(\mathrm{m}) \\
\mathrm{y}^{+} & =\text {dimensionless wall distance } \\
\mathrm{Y}_{\mathrm{M}} & =\text { compressibility correction factor }
\end{array}
$$

\begin{tabular}{|c|c|c|}
\hline Symbol & & Definition \\
\hline$\alpha$ & $=$ & angle of attack $\left(^{\circ}\right)$ \\
\hline$\beta$ & $=$ & coefficient of thermal expansion \\
\hline$\beta^{\prime}$ & $=$ & 3-D lift curve approximation term \\
\hline$\gamma$ & $=$ & ratio of specific heats \\
\hline$\delta_{\mathrm{f}}$ & $=$ & flap deflection $\left(^{\circ}\right)$ \\
\hline$\delta_{\mathrm{ij}}$ & $=$ & Kronecker delta \\
\hline$\Delta$ & $=$ & increment \\
\hline$\varepsilon$ & $=$ & turbulence dissipation rate \\
\hline$\varepsilon^{\prime}$ & $=$ & absolute error \\
\hline$\eta$ & $=$ & calibrated model constant term \\
\hline$\theta$ & $=$ & departure angle from flap upper surface $\left(^{\circ}\right)$ \\
\hline$\kappa$ & $=$ & curvature \\
\hline$\kappa^{\prime}$ & $=$ & von Karman constant \\
\hline$\Lambda_{(\mathrm{c} / 4)}$ & $=$ & quarter-chord wing sweep angle $\left(^{\circ}\right)$ \\
\hline$\mu$ & $=$ & dynamic viscosity $\left(\mathrm{kg} /\left(\mathrm{m}^{*} \mathrm{~s}\right)\right)$ \\
\hline$\mu_{\mathrm{t}}$ & $=$ & turbulent viscosity term \\
\hline$\mu_{\mathrm{T}}$ & $=$ & turbulent viscosity $\left(\mathrm{kg} /\left(\mathrm{m}^{*} \mathrm{~s}\right)\right)$ \\
\hline$v$ & $=$ & kinematic viscosity $\left(\mathrm{m}^{2} / \mathrm{s}\right)$ \\
\hline$\rho$ & $=$ & density $\left(\mathrm{kg} / \mathrm{m}^{3}\right)$ \\
\hline$\sigma_{\varepsilon}$ & $=$ & turbulent Prandtl number \\
\hline$\sigma_{\mathrm{k}}$ & $=$ & turbulent Prandtl number \\
\hline$\tau_{\mathrm{ij}}$ & $=$ & viscous stress tensor \\
\hline$\tau_{\mathrm{w}}$ & $=$ & wall shear stress $\left(\mathrm{N} / \mathrm{m}^{2}\right)$ \\
\hline$\Phi$ & $=$ & value of solution \\
\hline$\Phi_{\infty}$ & $=$ & value of solution of infinite cell mesh \\
\hline$\omega$ & $=$ & specific dissipation rate \\
\hline$\omega_{\mathrm{k}}$ & $=$ & angular velocity $(\mathrm{rad} / \mathrm{s})$ \\
\hline$\Omega_{\mathrm{ij}}$ & $=$ & mean rate-of-rotation tensor \\
\hline
\end{tabular}

\section{Greek Symbols}




\section{Subscripts}

$\begin{array}{lll}\text { Symbol } & \text { Definition } \\ \infty & =\text { freestream value } \\ i, j, k & =\text { Cartesian indexing term } \\ \text { slot } & =\text { value at location of blowing slot } \\ \text { stag } & =\text { stagnation value } \\ \text { target } & =\text { target design value }\end{array}$

\section{Acronyms}

\section{Symbol}

AMELIA

APU

CAD

$\mathrm{CC}$

CCR

$\mathrm{CCW}$

CESTOL

CFD

DR

DWTNSRDC

GCI

GTRI

NASA

PR

QSRA

RANS

RSRA

SST

STOL

USB

VTOL

WVU

\section{Definition}

$=$ Advanced Model for Extreme Lift and Improved Aero-acoustics

$=$ auxiliary power unit

$=$ computer-aided design

$=$ circulation control

$=$ circulation control rotor

$=$ circulation control wing

$=$ cruise efficient short takeoff and land

$=$ computational fluid dynamics

$=$ dual radius

= David W. Taylor Naval Ship Research and Development Center

$=$ grid convergence index

$=$ Georgia Technical Research Institute

= National Aeronautics and Space Administration

$=$ prescribed radius

$=$ Quiet Short-haul Research Aircraft

$=$ Reynolds Averaged Navier-Stokes

$=$ Rotor Systems Research Aircraft

$=$ shear stress transport

$=$ short takeoff and land

$=$ upper surface blowing

$=$ vertical takeoff and land

$=$ West Virginia University 


\section{Chapter 1: Introduction}

\section{Motivation}

With the strong shift towards 'greener' technologies in today's world, the aviation industry is being pushed even harder to achieve greater goals in efficiency, emissions, and noise. Airline industries are paying more attention to more drastic conceptual and innovative designs that hold the potential of achieving both these high environmental standards and yield performance that meets and even exceeds that of existing solutions. Though these new ideas, concepts, and designs can only be achieved with great research and development due to the long and stringent process of readying a technology from concept to commercial aviation. To attain the necessary momentum and funding for the required research, a new technology or design must first gain the interest and support of many.

Circulation control (CC) is a perfect example of this occurrence. Development of CC flap systems has long been part of a vision for next-generation subsonic transport aircraft, offering significant payoffs in both performance and system complexity ${ }^{1-6}$ but has yet to acquire the needed support and backing to flourish. The technology has been around for 75 years and is now gaining more momentum for further development due to increased airport congestion and environmental standards. Currently the main environmental concerns of the aviation industry are noise, emissions, and fuel efficiency. These factors impact airport size (runway length), flight patterns in the community surrounding the airport, cruise speed, and many other aircraft design considerations 
which are determining the requirements for next generation aircraft. Considering these elements of air travel, NASA has set goals for next generation regional airliners and has categorized the aircraft that meet these goals as Cruise Efficient Short Takeoff and Land (CESTOL) aircraft, as seen below in Figure 1-1. With CC technology addressing most of the above listed issues, it is gaining more interest.

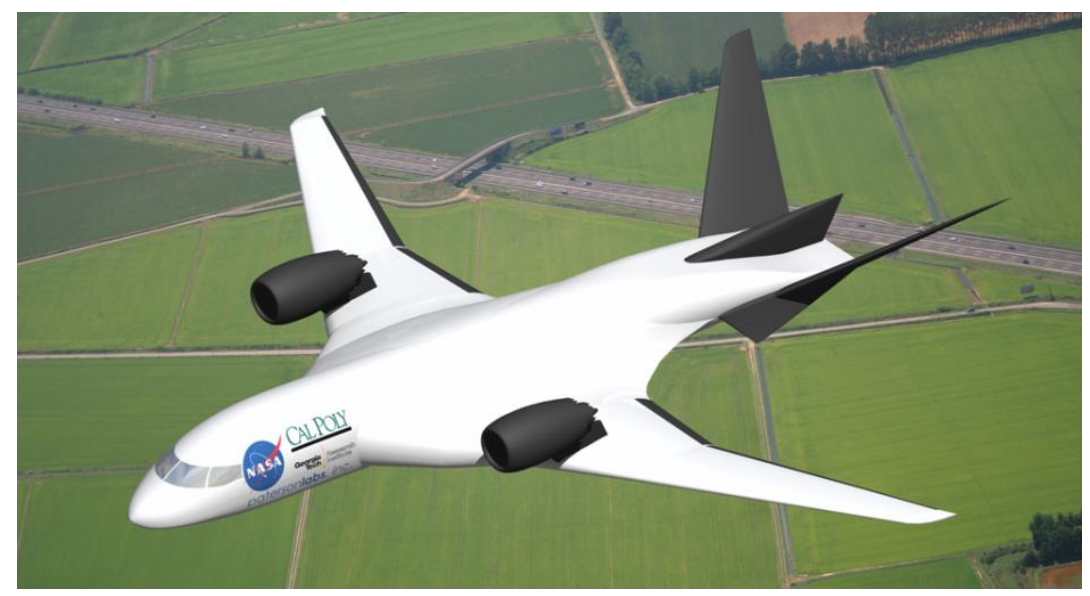

Figure 1-1: Cal Poly developed CESTOL aircraft ${ }^{7}$

The succeeding research was aimed at being a contribution to this movement, providing a useful database of information that can be utilized in future research and development of the $\mathrm{CC}$ technology. Although this characterization of the $\mathrm{CC}$ geometry was directed towards the application to a specific aircraft design, it contains many trends and ideas that can be used in $\mathrm{CC}$ flap design along with a multitude of other fields that could benefit from the pneumatic technology. With the persistent development of the current $\mathrm{CC}$ technology database, the opportunities for its future utilization are vast.

\section{Circulation Control Background}

The use of pneumatic devices in the form of blown jet airfoils in aerodynamics has been under investigation as far back as the 1930's, and possibly earlier. ${ }^{2,8,9}$ A 
Romanian inventor, by the name of Henri Coanda, discovered the phenomena of CC (claimed to be in 1910) in a near-fatal plane crash where the exhaust deflection plates he developed to deflect the exhaust away from the aircraft instead entrained the hot exhaust onto the aircraft, causing it to ignite. ${ }^{9}$ The past concepts involved ejecting a jet nozzle just upstream of a corner with distinct steps and angles. The purpose of this corner was to deflect the jet around the corner and entrain fluid from the other side of the jet nozzle over the side of the jet. These earlier concepts used distinct steps and angles to promote mixing of the air through the addition of turbulence. Throughout the development of CC devices the stepped surfaces have changed to smooth curving surfaces to decrease energy loss and increase the entrainment capability. ${ }^{8}$ The first person to use the CC concept for high lift generation was Davidson around 1960. ${ }^{10,11}$

The most common concept of circulation control applied to fixed wing applications involves ejecting a thin sheet of high momentum jet flow over a curved surface on the trailing edge of the airfoil. This jet flow is usually ejected out of a slot located just upstream of the trailing edge of an airfoil, which is depicted below in Figure 1-2. The high momentum air follows the curved surface due to both the sub-ambient pressure caused by the jet flow and the centrifugal force created by the curved/centrifugal surface turning away from the jet flow's initial direction, which keeps the flow attached to the curved surface through large angles of turning. This occurrence is also known as the Coanda effect. The airflow over the wing is then entrained over the curved surface by the pressure gradient caused between the lower pressure of the high momentum air and the higher pressure of the ambient air the airfoil is traveling through. This lowers both leading and trailing edge stagnation points to the bottom surface of the airfoil, increasing 
effective camber and circulation resulting in additional lift. Figure 1-3 presents the streamlines encountered during the operation of a CC flap, which shows the migration of the stagnation points to the lower surface. The increased circulation caused by CC flaps has been seen to produce 2-D lift coefficients $\left(C_{1}\right)$ upwards of 7 to $10 .^{12}$ It has been demonstrated that CC flap systems can produce substantial lift gains over the conventional flapped airfoil ${ }^{3,4}$ and produce high lift coefficients independent of angle of attack $(\alpha) .{ }^{13}$ The CC flaps can also be coupled with over-the-wing engine configurations to provide even greater lifting capability, which has been observed to produce engine thrust turning well past $90^{\circ}$ from the horizontal. ${ }^{2}$

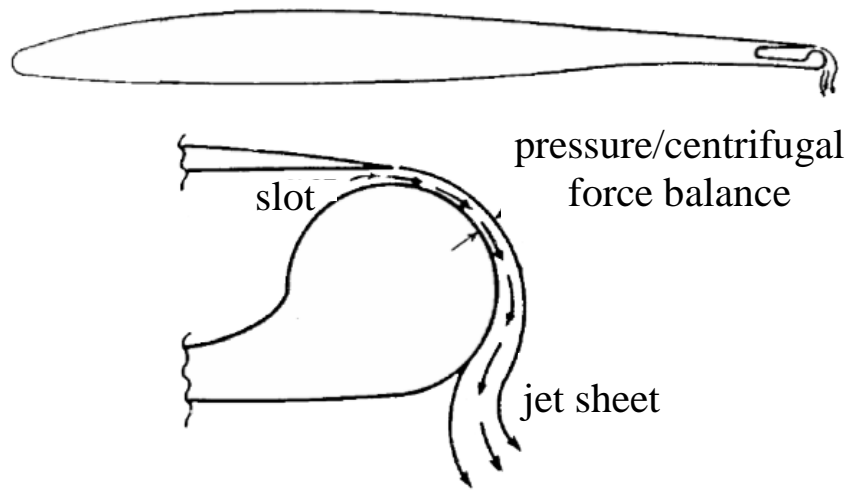

Figure 1-2: CC airfoil displaying the Coanda effect over trailing edge9

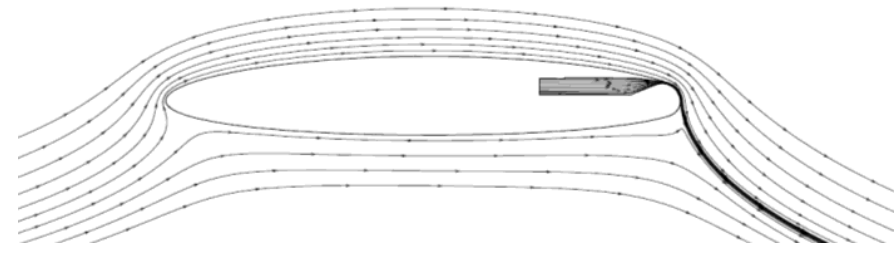

Figure 1-3: Streamlines during CC operation ${ }^{14}$

Although the increased circulation caused by this active control system does have very desirable lifting capabilities, there are several attributes of the concept that should be noted. The lowering of the leading and trailing edge stagnation points during $\mathrm{CC}$ 
operation makes the airfoil susceptible to leading edge stall, which has been experienced in flight tests of a CC flap system. ${ }^{5,15,16}$ Since the use of CC is most desirable for its high lifting capabilities at low speeds during takeoff and land (low altitudes), leading edge stall could easily cause a catastrophic failure to the aircraft. This problem can easily be countered with the use of leading edge devices, which are currently in use on today's STOL aircraft. The use of a leading edge blowing slot in the prevention of leading edge stall has been an experimentally proven, and has shown even greater performance in increasing stall $\alpha$ over more complex mechanical leading edge devices. ${ }^{5,15}$

Another challenging aspect of the use of $\mathrm{CC}$ is the source of the jet flow that is ejected out of the slot. There have been several solutions that have been developed to solve this problem. The first option would be to bleed air from the existing power plant on the aircraft, though this would also impose another problem since the engine of the aircraft is sized for takeoff thrust required. With the operation of CC flaps also occurring during the aircraft's mission segment of takeoff, the use of a CC system on an aircraft would require increased sizing of the engines. The larger engine sizing would result in lower fuel efficiency during cruise, and with cruise being the longest mission segment of most aircraft, it would have a significant impact on the overall performance of the aircraft.

A second possible solution in generating compressed air for $\mathrm{CC}$ operation is a dedicated auxiliary power unit (APU). This APU would be an additional system that the aircraft would need to incorporate, but could also be used in flight for heating/air conditioning or pressurization when it isn't needed to power the flap system. ${ }^{17-19}$ With the APU, the engines will not encounter the thrust losses needed to feed the blowing slots 
and will be able to be sized appropriately to their mission to optimize the efficiency of the system.

\section{Previous Works/CC History}

There have been many different research programs which have explored the use of $\mathrm{CC}$ in aeronautical applications, as well as various other fields. These projects include experimental investigations, computational analysis, and flight test demonstrators. Most of these CC works have been concentrated on the application of the entire system, rather than extensive examination of the flap geometry's influence on $\mathrm{CC}$ performance. Although, many of these works have still contributed significant relations and findings that have influenced the flap design and shape. With the current progress, development, and accessibility of today's computing resources, there has been a significant increase in computational research which has also been promoting a broadening of research scope to more specialized topics. The following are examples of successful programs in the different fields of $\mathrm{CC}$ research that have contributed insight into the design of the flap surface geometry.

\section{Experimental Research}

The experimental research on $\mathrm{CC}$ development has been one of the richest of the past research fields. Most of this research has been used to extend the base knowledge of $\mathrm{CC}$ technologies and to convey the promising benefits they hold. One of the main contributors to the experimental research done on CC is the David W. Taylor Naval Ship Research and Development Center (DWTNSRDC) in Bethesda, MD. Their pursuit in research of this technology has produced significant advancements that have been applied 
to $\mathrm{CC}$ development in the fields of fixed wing aircraft, rotorcraft, and marine applications. $^{20,21}$

Most of the major efforts in the experimental research of $\mathrm{CC}$ fall into the following categories: circulation control rotor (CCR), circulation control airfoil development, circulation control wing $(\mathrm{CCW})$, and advanced circulation control airfoils. Each of the four categories provided relevant findings in regards to $\mathrm{CC}$ flap geometry design, although the categories of circulation control airfoil development and advanced circulation control airfoils contributed the most being more concentrated on the performance of the airfoil rather than the integration of the system.

\section{Circulation Control Rotor (CCR)}

CCR was one of the first areas of CC experimental research, performed during the mid-late 1960's in the field of rotorcraft. One of the major drivers of the CC technology in this field was its capability of high-lift independent of angle of attack, which would eliminate the required blade pitching mechanisms. ${ }^{2,22-24}$ Very influential parameters that help characterize CC performance were developed in these early CCR studies, which were slot height-to-chord ratio, slot height-to-trailing edge radius ratio, Reynolds number,

and jet Mach number. ${ }^{25}$ Of these parameters the slot height-to-trailing edge radius ratio had direct influence on the flap geometry design. The NASA X-Wing Rotor Systems Research Aircraft (RSRA) was a product of this research (Figure 1-4 below), which utilized a four-bladed CCR for short and vertical takeoff and landing that would stop rotation and be used as a wing for forward flight improving cruise efficiency and higher speed capability. ${ }^{22,26-29}$ The main benefits CC provided to rotor craft were its 
breakthroughs in rotor efficiency, parasite drag, weights, and system complexity, ${ }^{23}$ which then presented much potential for fixed-wing aircraft application.

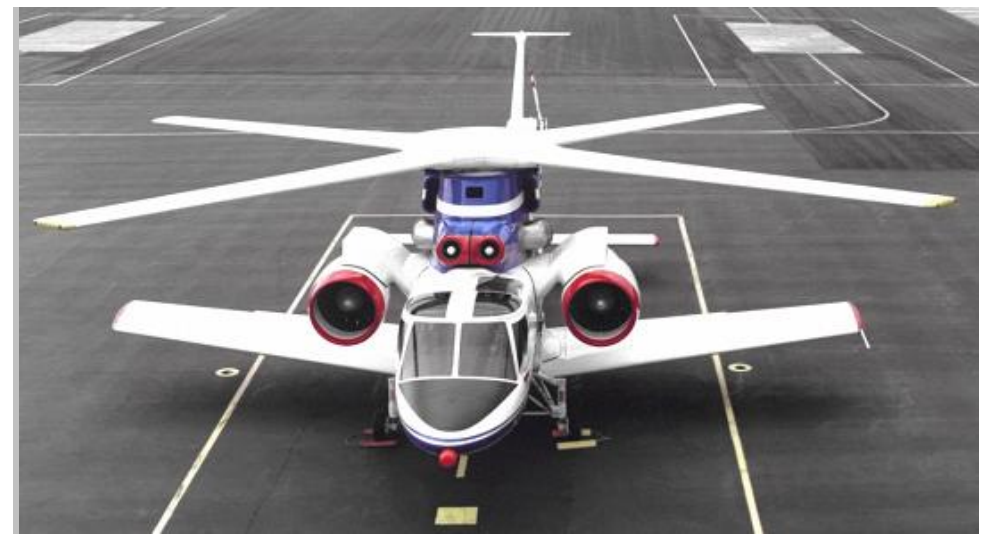

Figure 1-4: NASA's X-Wing RSRA flight demonstrator (with main rotor) ${ }^{29}$

\section{Circulation Control Airfoil Development}

The application of this $\mathrm{CC}$ technology quickly grabbed the interest of use as a flap-device to short takeoff and land (STOL) aircraft and research broadened to airfoils and wings as well as rotors. The main driver behind this push was that the technology offered great potential for STOL aircraft, being a simple high-lift device that could operate independent of angle of attack. Since CC technology was still very new at this time, most of the research performed in this application was done in order to observe general CC trends rather than to design or optimize it for a particular system. Much of this research was also performed in conjunction with the CCR development at the Navy's DWTNSRDC.

Symmetrical elliptical and semi-elliptical airfoils were utilized for most of the initial CC airfoil research similar to those used in CCR research, as seen in Figure 1-5. These elliptical-shaped airfoil studies produced a large amount of data on CC 
performance and trends, including results that suggested smaller slot heights and shorter Coanda surfaces generate greater lift and moment for subsonic freestream velocities and larger slots and longer surfaces generate larger lift and moment in transonic freestream velocities. ${ }^{30}$ Another characteristic that these CC airfoil studies displayed was that although shorter, highly curved Coanda surface configurations produced larger forces, the longer, smoother transitioning Coanda surface configurations were more effective over a broader range of conditions. ${ }^{30}$ The effect of the tailing edge blowing on flow field around the leading edge was also observed.

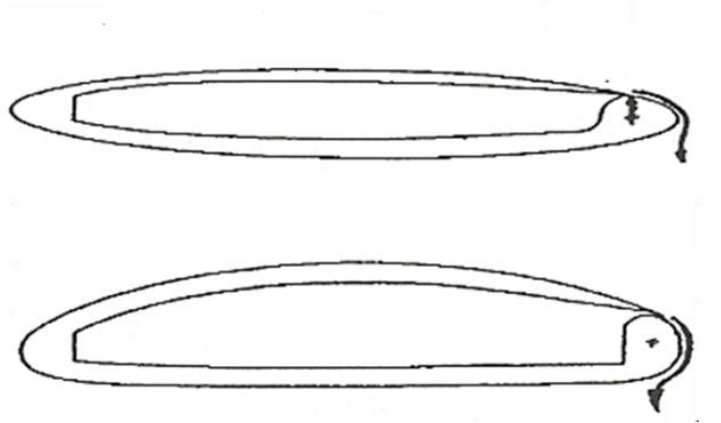

Figure 1-5: Elliptical and semi-elliptical CC airfoils ${ }^{2}$

The experimental development of CC airfoils greatly increased the understanding and performance of the $\mathrm{CC}$ technology, but also revealed several adverse aerodynamic characteristics of the early CC airfoils. Similar to the previous research in field of CCR, CC airfoils also experienced leading-edge separation with increased blowing or circulation around the airfoil. This could be countered with either mechanical leadingedge devices or pneumatically with a leading-edge slot, but was still seen as an aerodynamic weakness to the airfoil. The original CC airfoils also suffered by having large, blunt, circular trailing edges which greatly increased the drag of the airfoil when the $\mathrm{CC}$ was not in operation. The large negative pitching moments that accompanied the 
large lift forces was also seen as a problem, requiring a large tail surface to counter the nose-down moment if applied to an aircraft.

\section{Circulation Control Wing (CCW)}

With the on-going CC airfoil research showing large lift gains for very generic shaped airfoils, Coanda devices were soon being modeled for existing aircraft as a small add-on device that converted the sharp trailing edge to a rounded Coanda surface., ${ }^{2,5,31}$ Initial tests of these converted $\mathrm{CCW}$ configurations performed at the DWTNSRDC showed significant advantages of the $\mathrm{CCW}$ over previous blown flap configurations, being much greater streamline displacement and no sharp trailing edge to limit the streamline turning resulting in larger lifting capabilities. This, along with additional experimental research on the $\mathrm{CCW}$ configuration, proved that the $\mathrm{CCW}$ flap system was the most efficient of all the other blown flap configurations. ${ }^{32}$

A lot of useful results were obtained through the many experimental tests performed on $\mathrm{CCW}$ configurations, which included 3-D flow features encountered during CCW operation, development of parameter characterization, system integration, flight demonstrator support, and coupling with propulsion for even greater liftingperformance. . $^{2,31,33-44}$ The parameters that were found to have the most effect on the airfoil performance were leading edge devices, trailing edge configuration (radius, slot location, flap deflection), freestream Reynolds number, airfoil incidence, momentum coefficient, slot height, and nozzle pressure ratio at the jet. ${ }^{45}$ Experimental work on CCW that supported the A-6 $6^{46,47}$ and NASA Quiet Short-haul Research Aircraft $(\text { QSRA })^{35,36}$ flight and technology demonstrators even addressed the possibility of 
improving the cruise performance of the aircraft by modifying the flap geometry, using a smaller radius on the trailing edge of the $\mathrm{CCW}$.

\section{Advanced Circulation Control Airfoils}

Through the years of $\mathrm{CC}$ development in airfoil design and its application to aircraft, several unfavorable aerodynamic characteristics of the system had been uncovered. Those being leading edge separation, cruise drag, blowing air sources, and noise. Much effort of the research post $\mathrm{CCW}$ was to improve the traits of the $\mathrm{CC}$ system while maintaining the high lift augmentation. A large amount of this effort was performed by Robert Englar and the DWTNSRDC, developing solutions for several of these issues and incorporating them into the advanced CC airfoil.

The improvements to the $\mathrm{CC}$ airfoil, and leading up to the advanced $\mathrm{CC}$ airfoil, stemmed from the research of the CCW. The first significant improvement was discovered during experimental development of a CCW system for Naval purposes, when it was found that applying a smaller radius at the trailing edge of a $\mathrm{CC}$ airfoil would produce excellent lifting capabilities at high momentum coefficients and greatly improve

the cruise performance of the airfoil. ${ }^{35}$ The smaller radius allowed for a large angle of turning in a short chord-wise distance along with a much reduced trailing edge thickness, which significantly improved cruise drag over the existing CC airfoils while keeping the simplicity of the no-moving-parts system. ${ }^{37}$ The smaller radius allowed for less modification of the original airfoil the CC system was implemented onto. This smaller radius $\mathrm{CC}$ airfoil was shown to be a significant improvement over the airfoil of the A-6 flight test. ${ }^{48}$ Figure 1-6 below presents the improved smaller radius CC airfoil. 


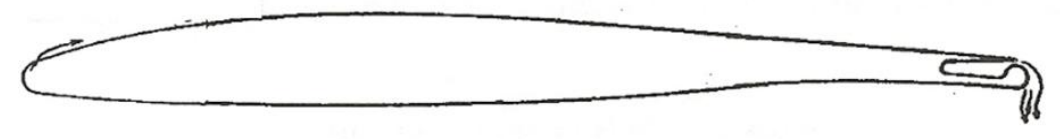

Figure 1-6: Improved small-radius CC airfoil $^{2}$

The next advancement of the CC airfoil was the type of airfoil utilized for its application. It was found that the use of a supercritical-type airfoil provided a large leading-edge radius to prevent leading edge separation for a range of angle of attacks having good low speed characteristics ${ }^{48}$ along with a thick bluff trailing-edge which provided sufficient volume for the radial surfaces of the trailing edge and the blowing plenums for the trailing edge slot. ${ }^{49}$ This large leading-edge radius eliminated the need for a leading edge device at low values of momentum coefficient, while also offering sufficient room for the integration of a mechanical or pneumatic leading edge device if larger momentum coefficients were desired. The supercritical airfoil also produced a much more desirable cruise performance over that of the earlier CC airfoils. ${ }^{37}$ The large leading and trailing edge thickness of the supercritical airfoil are depicted below in Figure 1-7.

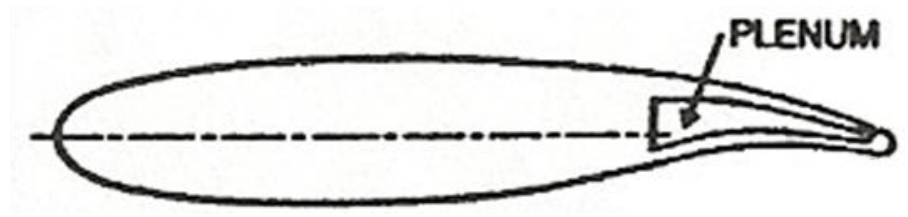

Figure 1-7: Supercritical airfoil with large leading edge and CC trailing edge ${ }^{2}$

One of the most significant advancements of the CCW airfoil has been the substitution of a small, rotatable flap located just aft of the blowing slot in the place of the non-moving, circular trailing edge. Developed by Robert Englar of DWTNSRDC, the small flap consists of two different radial arcs and is called the dual radius flap. The first 
radial arc is a $90^{\circ}$ arc that has a small radius, with the second radial surface having a substantially larger radius and ending with a sharp trailing edge. The concept of the dual radius flap was that when it is deflected, it utilizes the smaller radial surface to turn the slot flow over a greater angle in a shorter chord-wise distance while the slot flow has high momentum energy from the slot. The dual radius then uses the larger radial surface of the flap to keep the slot flow attached as the high momentum energy is reduced while traveling the flap surface. ${ }^{49}$ The dual radius flap can then be retracted exposing only the larger radial flap surface, which better contours the upper surface of the wing, and also employs a sharp trailing edge. This sharp trailing edge increases thrust recovery during $\mathrm{CC}$ operation, along with greatly reducing the pressure drag in the cruise configuration. ${ }^{49}$ An advanced CC airfoil employing a dual radius flap with leading and trailing edge blowing is displayed below in Figure 1-8.

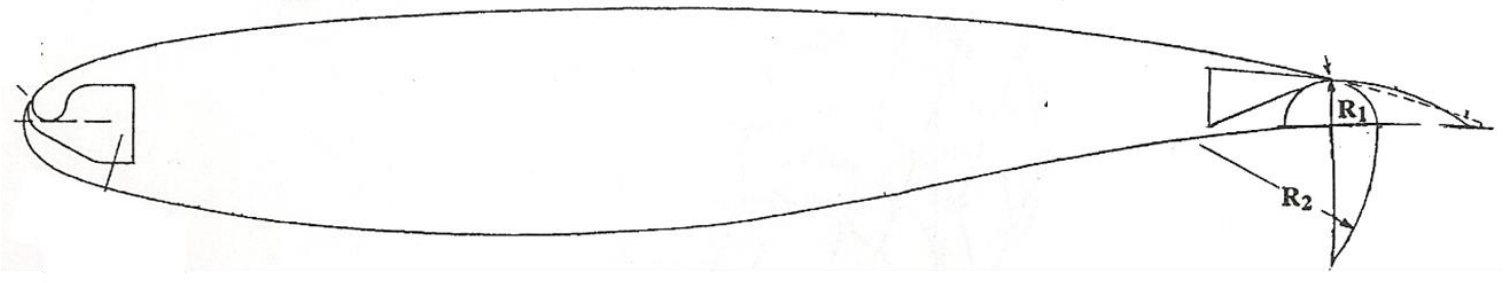

Figure 1-8: Advanced dual radius $\mathrm{CC}$ airfoil with leading edge blowing ${ }^{2}$

\section{Computational Fluid Dynamics (CFD) Research}

Computational research of $\mathrm{CC}$ has been an important part of fully understanding the flow features involved with the CC phenomenon. Past attempts of computationally modeling $\mathrm{CC}$ flow fields have fallen short in capturing all the complex flow characteristics, requiring additional effort to be put towards understanding all of the physics encountered during CC operation. With the current development and validation 
of CC CFD techniques reaching even closer to accurately simulating these flows, CFD is gaining more attention and momentum in the $\mathrm{CC}$ community as a very useful and accessible tool for analysis. Although most of the past research in the field of CFD has been in the validation of the analysis tool and not in the characterization of the flap surface geometry, the research still provides great insight on the flow physics of CC flaps which can then be associated to the influences of the actual flap geometry.

Many of the initial efforts in CFD were to validate the CC flow simulation of the early 2-D semi-elliptical CC airfoils, which had available experimental data. Most results showed mixed results and inconsistencies with experimental data, most commonly with the over prediction of lift. ${ }^{9,50,51}$ The over prediction of lift was most commonly caused by the delay in jet separation from the Coanda surface of the airfoil..$^{51-54}$ It was found that simulations of CC airfoil geometries that employed sharp trailing edges (rather than rounded) predicted jet separation better, ${ }^{51}$ but still over predicted lift and was not a solution for simulating the actual CC flow. The inability for these simulations to match experimental data had multiple causes, with the most significant causes being mesh refinement and turbulence modeling. Unfamiliarity with the details of the $\mathrm{CC}$ flow field lead to many of the early attempts to simulate $\mathrm{CC}$ fall short of the necessary mesh refinement to capture the complete phenomenon. Several studies had found that computational meshes required much more refinement in the regions of the Coanda surface of the airfoil, along with the shear layer between the jet flow and freestream flow to more accurately capture all of the $\mathrm{CC}$ flow interactions. ${ }^{50-52,54,55}$ With adequate refinement within these areas of complex $\mathrm{CC}$ flow features, the same studies saw great improvement towards matching experimental results. 
Addressing the issues experienced with the turbulence modeling of $\mathrm{CC}$ flows, a general trend was observed that most models degraded in performance with the increase of momentum coefficient. $52,53,56,57$ A more detailed analysis has found that this rapid drop-off in turbulence model performance occurred when the freestream flow around the airfoil was not completely attached and separated flow was present. ${ }^{53}$ One of the most significant causes of the inconsistencies with some turbulence models prediction of the flow field has been attributed to the model's failure to account for curvature effects that are present in the flow. ${ }^{56,57}$ These curvature effects can be accounted for in the turbulence model and have been found to more closely match experimental data, but at a significantly higher computational cost. ${ }^{56,57}$ Though even with the curvature effects accounted for, simple one- and two- equation turbulence models were still over predicting lift. Other causes of the lift over predictions have been tied to the turbulence models inability to account for nonlinear eddy viscosity, vortex breakdown, along with near-wall effects and turbulence. ${ }^{58,59}$

Although most simple one- and two-equation turbulence models have been unsuccessful in accurately predicting lift, there has been some cases of matching experimental data with these models with both incompressible ${ }^{54,60}$ and compressible solvers. ${ }^{1,53,61,62}$ It has also been found that adjusting the angle of attack of CFD geometry to match the stagnation point location of experimental data will result in good correlation pressure distribution with the experimental data. ${ }^{50}$ Even when quantitative data is not accurately predicted by turbulence models, most models still capture the general trends associated with CC. ${ }^{61}$ 
With continued research into turbulence modeling with $\mathrm{CC}$, models have recently been developed to overcome the shortcomings of earlier attempts. One of which efforts was by Travis Storm with the $v^{2}-f$ extension of the k- $\varepsilon$ turbulence model, where he explored the linear, nonlinear, and curvature corrected variations of the model. ${ }^{59} \mathrm{He}$ has been able to come very close to matching experimental data with these variations, and displays the promise of the more recent development on turbulence models and their simulation of CC flows.

CFD research has lead to the observation of an additional item that has helped in more accurately modeling the flow field. This is the proper modeling of boundary layer development in the jet flow from the upstream plenum as it exits the slot and proceeds over the Coanda surface and through the shear layer, which has shown to have significant influence on the solution. ${ }^{50,63}$ To account for this phenomenon, the boundary conditions at the slot location need to account for the velocity profile of the flow traveling through the plenum or the entire plenum can be modeled.

CFD techniques have made significant advancements from their first applications to the CC flow field. The improvements have helped in making CFD a more reliable and accessible design and development tool, which in turn is assisting further CC development. With proper modeling of the complex aerodynamics involved with CC, CFD is an accurate tool to characterize the flap geometry.

\section{Flight Testing}

Flight testing has been the smallest area of CC research, with only two fixed-wing flight demonstrators and one rotorcraft flight demonstrator being flown. Along with the 
three flight tests of the technology there has also been one full-scale static test performed, which coupled with an upper surface blowing configuration with CCW for additional lift augmentation. Most of the technology demonstrators were modified from existing aircraft configurations rather than being designed with $\mathrm{CC}$ operation in mind, resulting in a less-than-optimal designs for the $\mathrm{CC}$ integration onto the specific aircraft. Even with design deficiencies, all of the tested flight demonstrators provided invaluable information pertaining to the application of $\mathrm{CC}$ systems and actual full-scale flight performance data of the designed CC flap geometries. Although there has not been much recent work in CC flight testing research, the previous tests have provided great insight into the technologies' application, feasibility, and potential with future aircraft systems.

\section{West Virginia University BD-4}

The first of the fixed-wing CC flight demonstration efforts was completed by West Virginia University (WVU) with a CC modified BD-4 kit plane in 1974. ${ }^{2,44,64}$ The aircraft employed leading edge blowing and a deployable circular trailing edge Coanda surface. The initial wing/CCW system of the aircraft was redesigned throughout the effort, utilizing a circular trailing edge that folded out (increasing wing area), a drooped leading edge, higher pressure ducting for more uniform slot flow, and boundary layer suction at the hinge of the unfolding trailing edge flap that provided air for the $\mathrm{CC}$ blowing. ${ }^{10,65}$ The BD-4 aircraft achieved the following design objectives with its CCW: low drag and high wing-loading for cruise configuration, variable drag and low wingloading STOL configuration, high-lift coefficient at low blowing coefficient (trimmed aircraft lift coefficient of 5.1 at momentum coefficient of 0.17 ), good pilot visibility (level flight attitude), low duct volume and low pressure losses, prevention of flap stall by 
suction at flap hinge, prevention of leading-edge stall through the use of drooped leading edge, and very rapid forward folding flap for conventional flight. ${ }^{65}$ Several other design features of the BD-4 flight demonstrator were the aerodynamic fences used between blown and non-blown junctions for aerodynamic and structural purposes, a 200 HP APU to power the $\mathrm{CC}$ blowing slots, and blown ailerons that could be drooped for additional lift. $^{18}$ A picture of the BD-4 in flight is presented below in Figure 1-9.

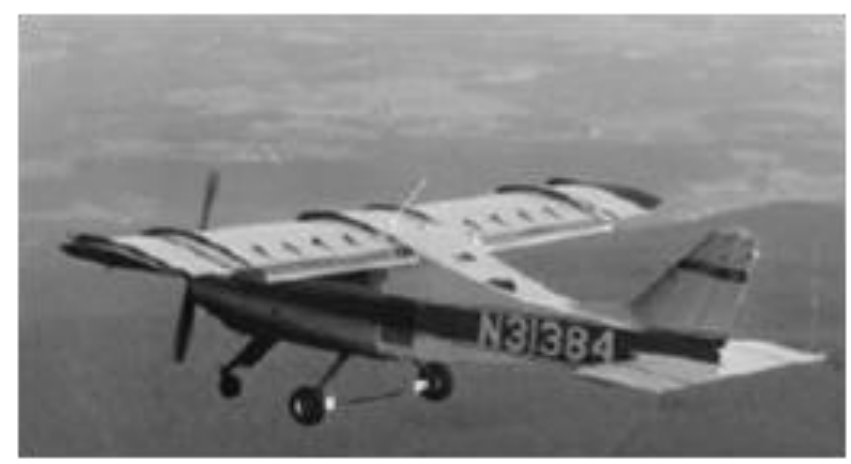

Figure 1-9: WVU BD-4 CCW flight demonstrator ${ }^{65}$

\section{Grumman A-6/CCW}

The following CC flight demonstration was performed by the Navy in 1979, flying a CCW retrofitted Grumman A-6., The aircraft was equipped with a completely circular non-retractable CC trailing edge, ${ }^{10,34}$ slots that were powered by engine bleed air, ${ }^{66}$ outboard aerodynamic fences, increased radius leading edge slats that were locked in a $25^{\circ}$ deflected position to prevent leading edge stall, ${ }^{5}$ a fixed Krueger leading edge flap that was added at the wing glove, wingtip jets to improve adverse yaw tendencies, ${ }^{67}$ as well as an inverted leading edge droop added to the horizontal stabilizer to counter the increased downwash. ${ }^{47,67}$ Numerous 2-D and 3-D wind tunnel tests and feasibility studies were conducted,,$^{2,5,46}$ one of which demonstrated a full $180^{\circ}$ of jet turning on a $1 / 8$ scale A-6 model in the DWTNSRDC tunnel. ${ }^{34}$ 
After the development and integration of the CCW system onto the A-6 aircraft, the A-6 was put through a series of ground, simulator, and flight tests (shown in Figure 1-10 below). In accomplishment of all of its testing objectives, the CCW modified A-6 demonstrated the optimistic characteristics of a $\mathrm{CCW}$ system. The results of the flight testing showed the following: a $140 \%$ increase in maximum useable lift coefficient during takeoff/approach angles of attack, 30-35\% reduction in liftoff/approach speeds, $60-65 \%$ reductions in takeoff/landing ground roll distances, and a $75 \%$ increase in payload/fuel at typical operating weight..$^{2,9,67,68}$ The overall findings of the A-6/CCW test program showed the potential of the technology for naval application with significant reductions in takeoff and landing velocities and distances, alternative for increasing aircraft's payloads, increased aircraft lifespan due to reduced kinetic energy and impact during aircraft carrier landings, increased pilot reaction times due to lower approach velocities, increased pilot visibility at lower angles of attack, and provision of a simple and relatively light weight STOL high lift system. ${ }^{34,68}$

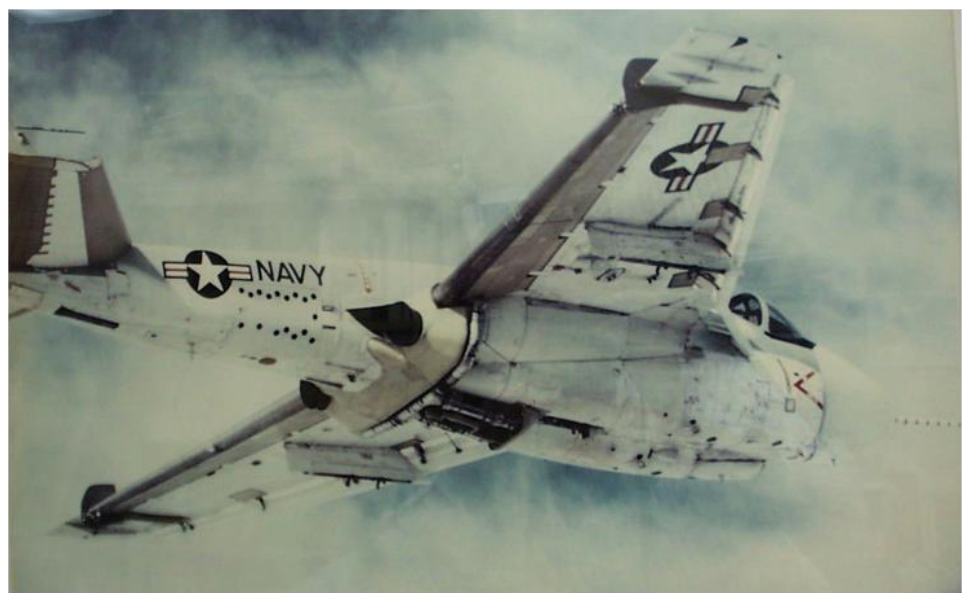

Figure 1-10: Flight testing of the A-6/CCW aircraft ${ }^{33}$ 


\section{$\underline{\text { Kaman XH-2/CCR }}$}

In 1975 Kaman Aerospace Corporation was awarded a contract from the DWTNSRDC to develop, design, and build the first CCR flight demonstrator, the Kaman XH-2/CCR (picture in Figure 1-11). ${ }^{69}$ Kaman used previous research and development in the CCR parameters of slot height-to-chord ratio, slot height-to-trailing edge radius ratio, Reynolds number, and jet Mach number to design of an improved CCR for the flight vehicle. ${ }^{70}$ The first flight of the test vehicle did not come until September 1979, but it successfully demonstrated the application and potential of CC to rotorcraft. ${ }^{69}$

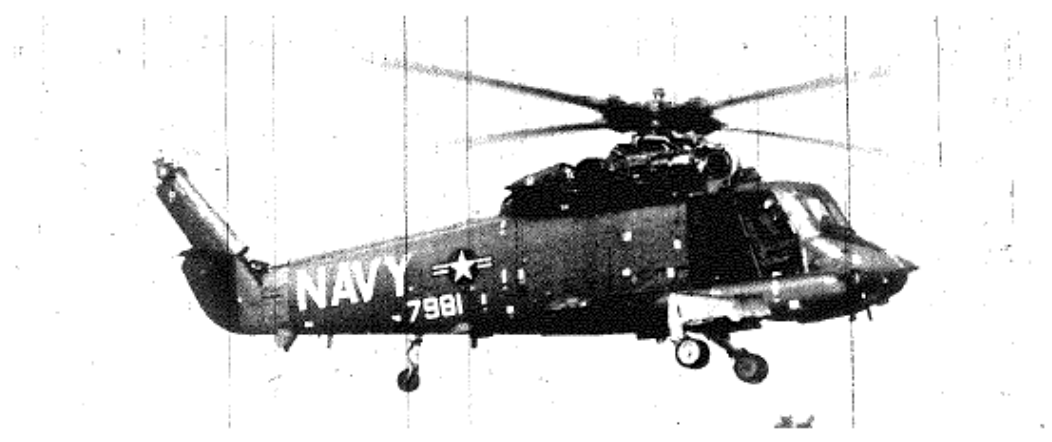

Figure 1-11: Kaman XH-2/CCR during hover flight test ${ }^{69}$

The flight tests proved that pneumatic aerodynamic and control systems can be used over the conventional mechanical cyclic and collective blade pitch control systems. The CCR blown rotor airfoil produced maximum lift coefficients in the order of 4-5, where conventional rotor airfoils only achieved lift coefficients of 1.4 , showing great possibility for use in heavy-lifting rotorcraft. The CCR also produced less drag than conventional rotors at the same lift coefficients, and showed promise in using higher harmonic control (more than once per revolution) to eliminate rotor-induced vibrations. ${ }^{69}$ 


\section{NASA Quiet Short-Haul Research Aircraft (QSRA)}

The USB/CCW modified NASA QSRA was not flight tested, but went through two phases of static ground testing which displayed significant improvement in design and integration. The concept of the aircraft was to use a CCW to entrain the engine exhaust of an upper-surface-blowing (USB) configuration as well as the airflow traveling over the wing, which had been previously pursued experimentally with several wind tunnel tests performed by DWTNSRDC. ${ }^{2,9,36}$ During the first phase of the investigation in 1981, the NASA research aircraft was outfitted with small, circular, no-moving-parts trailing edges to replace the complex existing flap system. The static tests resulted in thrust turning from $40^{\circ}-97^{\circ}$, and showed how the system could quickly transition from a high-drag configuration to a thrust recovery with the varying of the slot blowing. ${ }^{39}$ Two years later in 1983 the second phase of static testing took place with significant improvements in the $\mathrm{CCW}$ system design, which were five new trailing edge configurations, a larger CC blowing span, smaller slots, aerodynamic fences to help control the thrust turning, and larger radii on the Coanda surfaces. ${ }^{31}$ The improved design proved to increase the efficiency of thrust turning, with similar thrust turning to phase one results at considerably lower blowing momentum. The second phase configuration increased the thrust turning range slightly to $40^{\circ}-104^{\circ}$ depending on the thrust levels. The best performing trailing edge configuration was found to be the $90^{\circ}$ circular arc, achieving similar thrust turning of the larger arcs with lower blowing momentum along with minimizing the cruise-performance drag penalty due to the geometry of the no-moving-parts flap surface. ${ }^{42}$ The QSRA is displayed below in Figure $1-12$, pictured during one of its static tests. 


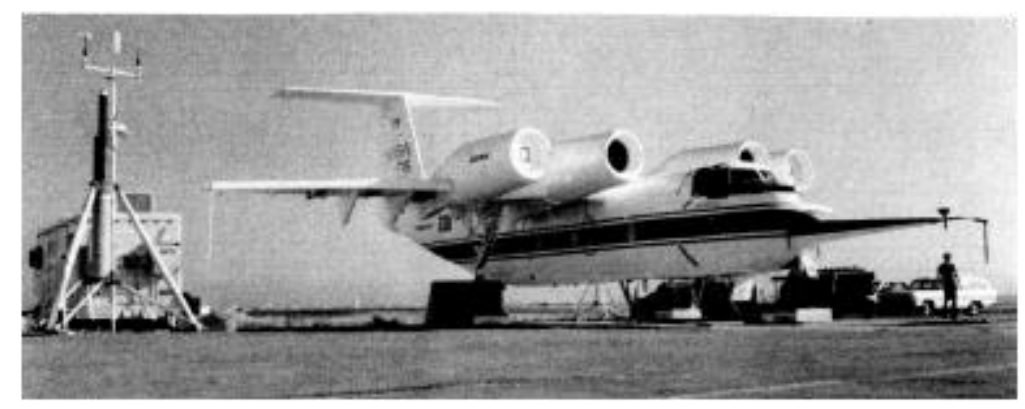

Figure 1-12: Static tests of the QSRA USB/CCW ${ }^{31}$

The noise impacts of the USB/CCW configuration of the QSRA was also investigated, which found the high frequency noise of the CCW was generally inaudible to surrounding communities and the $\mathrm{CC}$ of the configuration had no negative effect on noise levels. $^{41}$ Several feasibility studies followed the successful static USB/CCW tests, demonstrating the many benefits of the STOL configuration and its application to naval applications. ${ }^{33,40,43}$ One of the feasibility studies used results from powered model wind tunnel tests to show takeoff ground roll predictions as short as 100-200 feet, with powered lift coefficient values up to $8-9 .^{40}$ Although flight tests of the USB/CCW configuration have yet to take place, the results from both the static tests and feasibility studies still show the significant STOL benefits of the CC technology when coupled with the proven USB configuration.

\section{Direction of Work}

Through the pursuit of readying CC technology for industry application, significant progress has been made in the many different fields of CC. In continuation of $\mathrm{CC}$ research, this investigation will address the effects of the geometric parameters of the blown flap surfaces on the aerodynamics and performance of the CC system using twodimensional CFD as the analysis tool. This will provide guidance and insight on the 
geometric influences of the CC flap system, which has had little previous work. ${ }^{71,72}$ This work will also add to the public database of 2-D CFD results that can either be compared to future or previous studies or validated experimentally.

The two different $\mathrm{CC}$ flap geometries that were investigated were the dual radius flap, which was an existing flap design with analytical and experimental data available, and the prescribed radius flap, which was a design developed in this research to offer a variation of the dual radius flap to explore possible improvements of the new shape. Several of each flap's geometric parameters were varied, creating a design space to map the performance of each flap at three distinct flight conditions. The flight conditions were chosen to be equivalent to the takeoff, approach/landing, and transonic cruise mission segments that correspond with CESTOL goals. This work was aimed at characterizing the geometrical parameters of each flap surface to advance the understanding of $\mathrm{CC}$ performance for a greater range of flight conditions than the technology has previously been applied to, primarily short takeoff and land (STOL) and vertical takeoff and land (VTOL) conditions. 


\section{Chapter 2: Problem Statement}

NASA has been a lead contributor in pushing the airline industry towards newer more efficient technologies. In doing so, NASA has developed goals for future airliner aircraft determined by their expected date of service. Table 2-1 below displays these specific goals along with their expected deployment.

Table 2-1: NASA subsonic airliner performance goals for future aircraft ${ }^{19,73}$

\begin{tabular}{|c|c|c|c|c|}
\hline Generation & $\mathbf{N}$ & $\mathbf{N + 1}$ & $\mathbf{N + 2}$ & $\mathbf{N + 3}$ \\
\hline Description & $\begin{array}{c}\text { Boeing 787, Airbus } \\
\text { A350 }\end{array}$ & $\begin{array}{c}\text { CESTOL Tube \& } \\
\text { Wing }\end{array}$ & $\begin{array}{c}\text { CESTOL Hybrid } \\
\text { Wing Body }\end{array}$ & Beyond \\
\hline $\begin{array}{c}\text { Initial } \\
\text { Operational } \\
\text { Capability }\end{array}$ & $2008-2010$ & $2012-2015$ & $2018-2020$ & $\sim 2025$ \\
\hline Noise & - & $-32 \mathrm{~dB}$ & $-42 \mathrm{~dB}$ & $-71 \mathrm{~dB}$ \\
\hline NOx Emissions & - & $-60 \%$ & $-75 \%$ & better than $-75 \%$ \\
\hline $\begin{array}{c}\text { Aircraft Fuel } \\
\text { Burn }\end{array}$ & - & $-33 \%$ & $-50 \%$ & better than $-70 \%$ \\
\hline \begin{tabular}{c} 
Field Length \\
\hline
\end{tabular} & - & $-33 \%$ & $-50 \%$ & $\begin{array}{c}\text { exploit metro-plex } \\
\text { concepts }\end{array}$ \\
\hline
\end{tabular}

Technologies to meet these N+2 goals of NASA are currently being investigated by California Polytechnic State University under a NASA research grant. ${ }^{7}$ This research grant, titled "Advanced Model for Extreme Lift and Improved Aero-acoustics" (AMELIA), addresses the next-generation goals of NASA by validating CFD's predictive capability on next-generation technologies with a large-scale wind tunnel test that is scheduled to take place in the fall of 2011. The aircraft configuration for this grant is a CESTOL 100 passenger regional airliner, developed by David Hall. In acknowledging that the CESTOL airliner being developed was only one component of a very large 
transportation system, aspects from each component were considered in the configuration development of the aircraft to ensure the improvement of not only the aircraft, but the entire system as well. In doing so, the following transportation system components were taken into account: airport terminals, ground and aircraft traffic control, aircraft servicing and maintenance, and the aircrafts themselves. ${ }^{19}$ In specific, the aircraft goals sculpting the design of the aircraft component are based on noise, emissions, field length, flight patterns in the airport environ, and cruise speed performance metrics presented in Table 2-2 below. The next-generation technologies incorporated into this configuration were CC flaps, over-the-wing engine placement, engine nacelles with chevrons, and a hybrid blended wing body. A three view of the AMELIA design is displayed in Figure 2-1 below.

Table 2-2: NASA goals for $\mathrm{N}+2100$ passenger airliner ${ }^{74}$

\begin{tabular}{|c|c|}
\hline$\rightarrow$ & Balanced field length of 2,000 feet \\
\hline$\rightarrow$ & Efficient cruise at Mach 0.8 \\
\hline$\rightarrow$ & Takeoff and land speeds less than 50 knots \\
\hline$\rightarrow$ & Turn radius in terminal area less than $0.25 \mathrm{~nm}$ \\
\hline$\rightarrow$ & $1400-2000$ mile range \\
\hline$\rightarrow$ & Noise footprint within the airport boundary \\
\hline
\end{tabular}
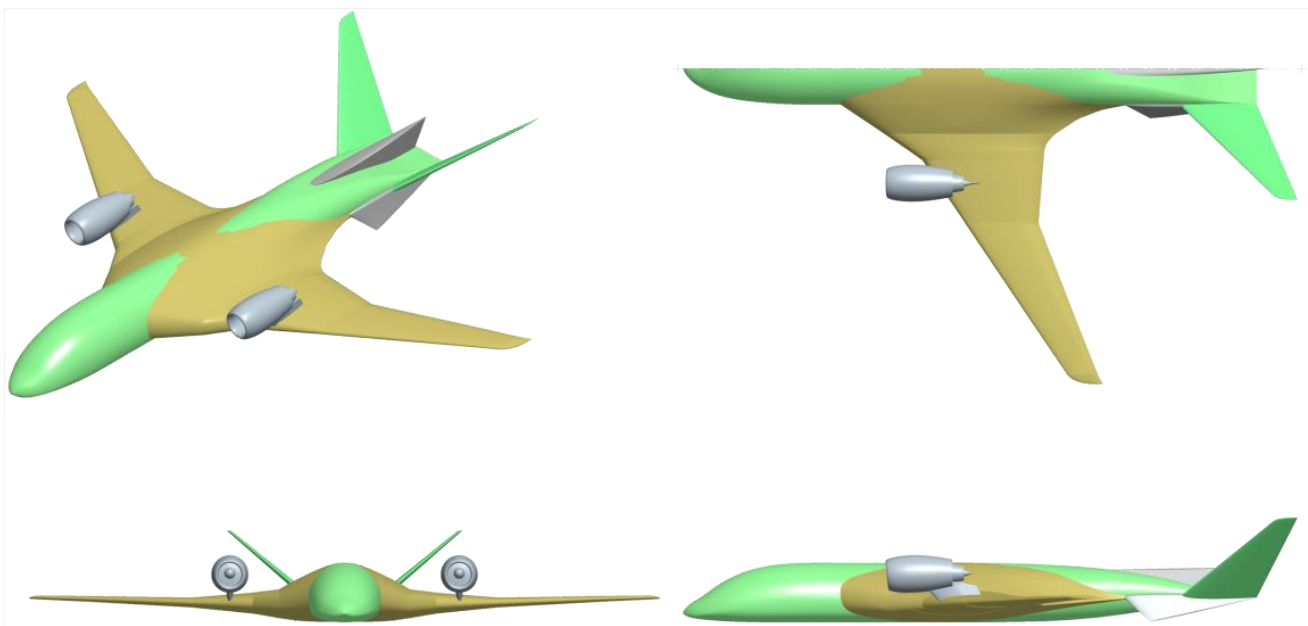

Figure 2-1: Three-view of AMELIA model 
The decision for CC flaps as the high lift technology on the AMELIA aircraft was chosen due to the increased efficiency of the CC flaps compared to other blown high lift systems, using the $\Delta \mathrm{C}_{1} / \Delta \mathrm{C}_{\mu}$ parameter as criteria (presented in Figure 2-2 below). The AMELIA wing also incorporates leading edge blowing to prevent upper surface separation of the airfoil, which has been experienced in flight tests and experiments of CC flap systems. ${ }^{5}$ The leading edge slot has been proven experimentally ${ }^{15}$ and has shown greater performance in increasing stall $\alpha$ over more complex mechanical leading edge devices. ${ }^{1}$ The CC slot air for this configuration will be provided by an APU, which will not require engine bleed to feed the slots which would result in thrust losses during flap operation and oversize the engines. ${ }^{17,18}$ This APU could also be used for heating/air conditioning or pressurization when it isn't needed to power the flap system. ${ }^{17}$ The CC flap system employed on the AMELIA design incorporates slots that retract when the system is not in operation to provide decreased cruise drag, which is important for optimizing fuel burn. ${ }^{10}$ The $\mathrm{CC}$ flaps provide many benefits to the AMELIA design; a less complex flap system, increased lift from over-the-wing engine exhaust entrainment, and a better performing flap that will result in shorter takeoff and landing distances, steeper climb-out and approach angles that reduce community noise, and decreased cruise drag. The use of CC flaps have also shown to be more acoustically acceptable than conventional mechanical flaps, ${ }^{75}$ by eliminating the vortex shedding in the trailing edge region of the airfoil. 


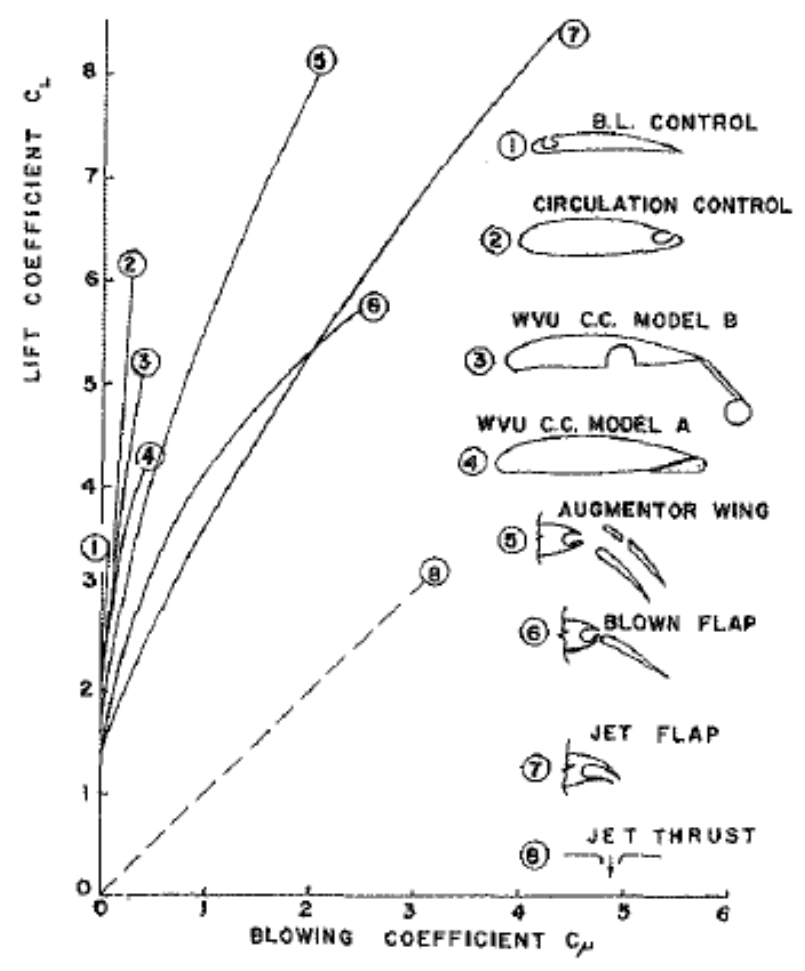

Figure 2-2: Lifting efficiency for various blown high-lift systems ${ }^{32}$

In the decision to use CC flaps for the AMELIA design, better performing flap geometries were desired. Past research has explored many of the other parameters involved with CC, like slot height, slot Mach, and operating conditions, though little research had been directed on the detailed geometry of the Coanda surfaces themselves. Although the main purpose of the CC flap system on the AMELIA aircraft was to provide high lift and drag for STOL operations, the system also had to facilitate lofty efficiency requirements of the cruise mission segment to meet the fuel consumption and emission goals set out by NASA. This drove the following research to not only find proficient high-lift CC flap geometry, but flap geometry that would also perform well in all the other mission segments of the aircraft. 


\section{Chapter 3: Methodology}

\section{Selection of Airfoil}

Since NASA's concerted effort to improve the performance of turbulent supercritical airfoils in the 1960's and 1970's, resulting supercritical airfoils have been able to provide good transonic behavior while retaining acceptable low-speed characteristics. $^{76}$ These airfoils achieved favorable transonic performance with the large leading edge radius, low curvature along the middle of the airfoil, cusp near trailing edge, and the same upper and lower surface slope at the trailing edge of the airfoil. At transonic speeds, the large leading edge radius causes strong expansions that will reflect back onto the airfoil as compression waves. Relatively small curvature along the midchord of the airfoil reduces the acceleration of flow, lessening the strength of the shock and associated large adverse pressure gradient. This shock-induced pressure gradient coupled with the typical aft-airfoil pressure gradient can easily cause boundary-layer separation on the airfoil surface, which results in increased drag as well as buffeting and stability problems. The trailing edge of the supercritical airfoil is at a small included angle with same upper and lower surface slopes to produce a slightly positive pressure coefficient at the trailing edge. This reduces the total pressure rise the upper surface boundary layer must transition to, helping to prevent separation. Some of these developed supercritical airfoils have significant thickness which slightly increases cruise drag, though enables the airfoil to reach higher maximum lift coefficients along with demonstrating docile stall behavior greatly increasing their low-speed capabilities. ${ }^{76} \mathrm{~A}$ NASA improved supercritical airfoil is displayed below in Figure 3-1. 


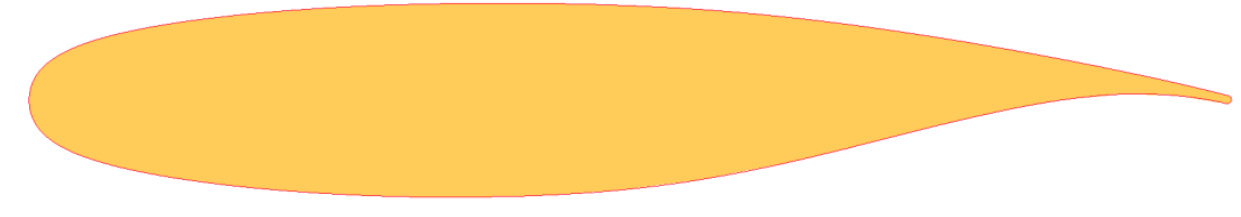

Figure 3-1: NASA SC(2) - 0414 airfoil

With the airfoil geometry and good low-speed characteristics, these NASA developed supercritical airfoils have been the most successful in the integration of CC flaps. The large leading edge radius helps in keeping flow attached during CC operation, and provides sufficient space for any leading edge devices. The trailing edge thickness of the supercritical geometry accommodates both the trailing edge slot plenum along with the radial surfaces of the flap. ${ }^{49}$ The transonic cruise performance of the supercritical airfoil is also desirable, delaying shock-induced drag with the top contour of the airfoil. ${ }^{76}$

In order to achieve AMELIA's design cruise lift coefficient along with the volume requirements of the CC system, the NASA SC(2)-0414 airfoil was chosen (picture above in Figure 3-1). The $\mathrm{SC}(2)$ of the airfoil designation represents the supercritical (SC) type of airfoil and phase (2) of NASA supercritical airfoil development. The first two of the four digits designate the design lift coefficient (0.4), and the last two digits designate the airfoil's thickness ratio (14\%). This airfoil was chosen due to its design lift coefficient being slightly above AMELIA's 2-D design cruise lift coefficient of 0.32 . This thicker airfoil (14\%) also provided sufficient volume for the CC systems, wing structures, and fuel storage. 


\section{Baseline Dual Radius Design}

Of the current CC flap configurations that have been developed (displayed in Figure 3-2), the dual radius will be the configuration further investigated. The dual radius flap first acquires the benefit of the smaller radius by turning the slot flow over a larger angle in a smaller chord-wise distance, which occurs because of the high momentum the flow still carries from being ejected from the slot. It then takes advantage of the larger radius to keep the flow attached as it travels along the flap and its high momentum energy is reduced. ${ }^{3}$ The dual radius also employs a sharp trailing edge which increases the jet thrust recovery during deployment and operation, and also greatly reduces the pressure drag during cruise. ${ }^{3}$ These contributions render the dual radius the best performing CC flap configuration of the current designs, thus making it the baseline flap to be developed in the forthcoming analysis.

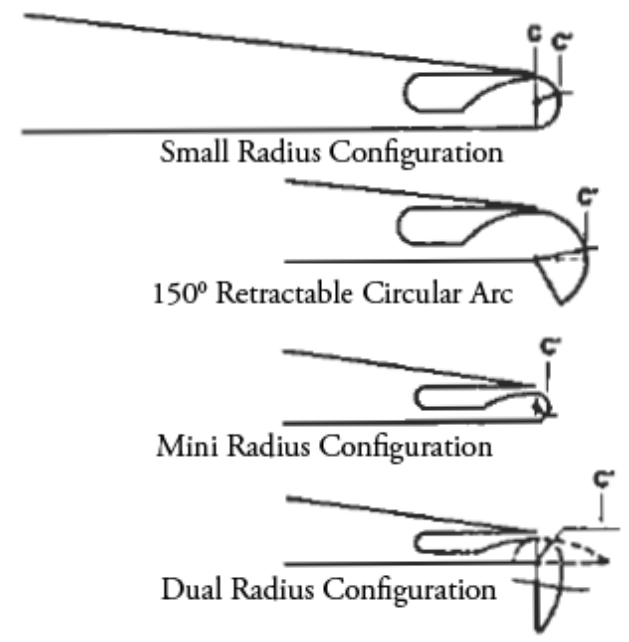

Figure 3-2: CC flap configurations ${ }^{12}$ 
Figure 3-3 below describes the defining geometry of the $\mathrm{CC}$ flap to be investigated.
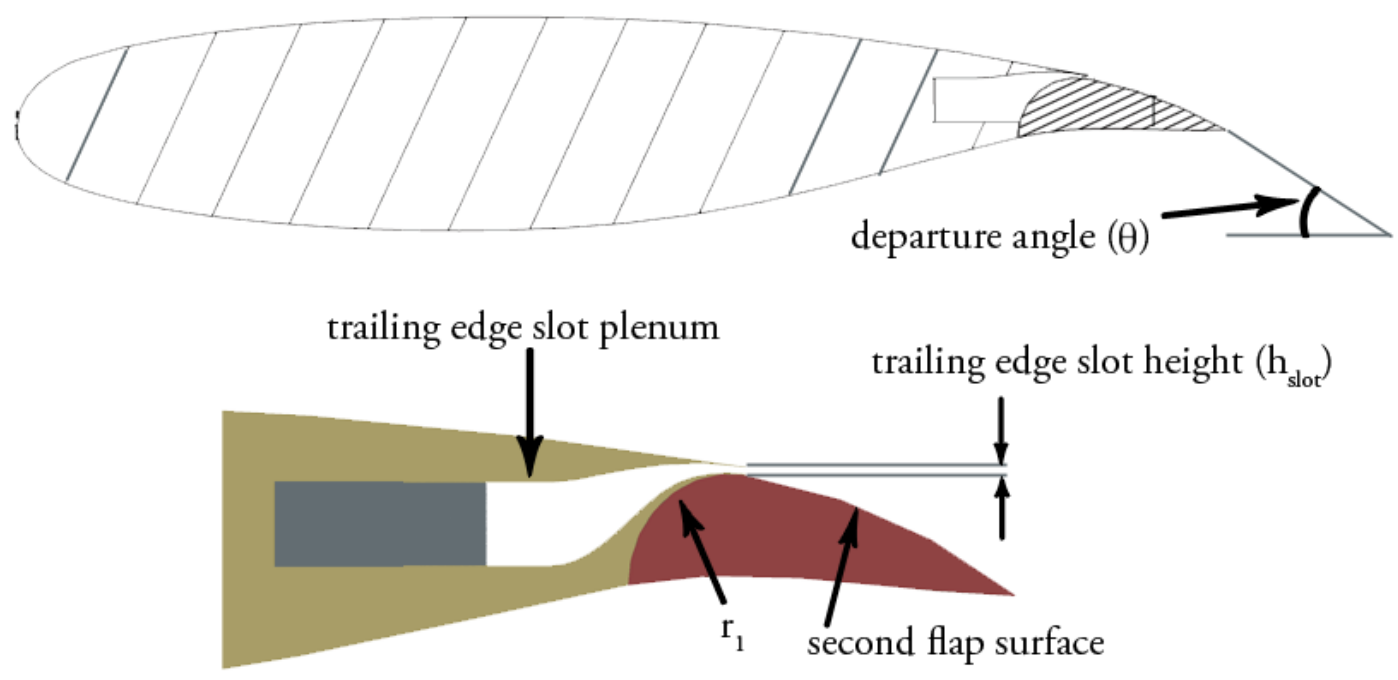

Figure 3-3: CC flap geometry investigated

Although the CC flap system of AMELIA incorporates a leading edge blowing slot to prevent upper surface separation of the airfoil at the high induced $\alpha{ }^{4}$ the investigated CC airfoil designs do not include a leading edge slot. Due to the importance of entraining the flow over the top of the wing using the high momentum air on the Coanda/flap surface, this design study explores the impact of solely the geometry of the flap on the aerodynamic performance of the CC flap system. By not including a leading edge slot in the analysis, it will isolate the performance characteristics of the $\mathrm{CC}$ system to be effected by only the changes in the aft flap geometry. The baseline $\mathrm{CC}$ dual radius flap configuration was designed to specifications developed by previous research. ${ }^{20}$ The chord of the $\mathrm{CC}$ airfoil was chosen to be the same length as the mean aerodynamic chord of the AMELIA design. In order to correctly capture the flow in the region of the flap, the slot plenum was also incorporated into the model. This allowed for proper flow 
characteristics of the slot flow, allowing the boundary layer to grow from the plenum inlet, throughout the slot, and onto the flap surface. The baseline flap parameters are presented in following sections.

\section{Slot Height and $1^{\text {st }}$ Radius}

The slot height and $1^{\text {st }}$ radius $\left(\mathrm{r}_{1}\right)$ of the $\mathrm{CC}$ system were determined from previous research performed for a naval report ${ }^{20}$ on the design of a CC application for submarine use, written by Englar and Williams. In this report, a working plot of several different CC parameters was constructed using experimental data. The parameters mapped in this plot were the slot height to chord ratio $\left(\mathrm{h}_{\text {slot }} / \mathrm{c}\right)$, slot height to flap radius ratio $\left(\mathrm{h}_{\text {slot }} / \mathrm{r}\right)$, and flap radius to chord ratio $(\mathrm{r} / \mathrm{c})$. This chart allowed an appropriate $\mathrm{h}_{\text {slot }} / \mathrm{c}$ to be chosen for a nominal range of $h_{\text {slot }} / r \leq 0.05$ (which yielded strongly attached Coanda jet flow) and for a range of $0.02 \leq \mathrm{r} / \mathrm{c} \leq 0.05$ (which yielded good flow turning without high suction peaks and high drag). This region of "most effective Coanda operation" is represented by the shaded region on the working plot below in Figure 3-4. 


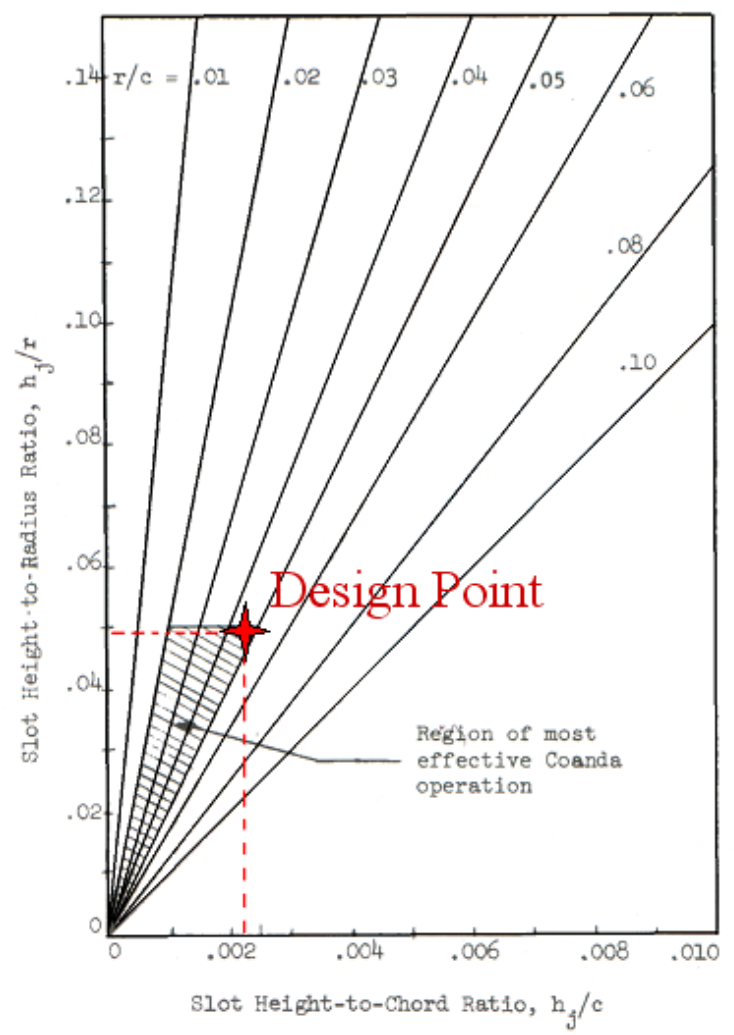

Figure 3-4: CC Coanda surface performance ${ }^{20}$

Although this previous work was performed on a single radius trailing edge CC foil, it was used to design $r_{1}$ of the current flap systems because, similar to the single radius trailing edge $\mathrm{CC}$ airfoil, $\mathrm{r}_{1}$ is the first surface that the slot flow will encounter. From guidance of the working plot above, the design $\mathrm{h}_{\text {slot }} / \mathrm{c}$ ratio of AMELIA was chosen to be 0.00238 . By choosing the upper-right corner of the shaded triangle of the plot, it allowed for the slot height to be larger to accommodate a reasonable size slot for the manufacturing of the AMELIA wind tunnel model without compromising the system's performance. With the selection of the $\mathrm{h}_{\text {slot }} / \mathrm{c}$ ratio, and using the above plot, the design $\mathrm{r}_{1} / \mathrm{c}$ ratio was also determined to be 0.04857 which kept the design point within the shaded triangle. A summary of all the baseline flap parameters are presented below in Table 3-1. 
Table 3-1: Baseline CC flap design parameters

\begin{tabular}{|c|c|}
\hline $\mathrm{c}(\mathrm{m})$ & 0.47370 \\
\hline $\mathrm{h}_{\text {slot }} / \mathrm{c}$ & 0.00238 \\
\hline $\mathrm{r}_{1} / \mathrm{c}$ & .04857 \\
\hline $\mathrm{h}_{\text {slot }} / \mathrm{r}_{1}$ & .04900 \\
\hline
\end{tabular}

\section{Momentum Coefficient $\left(C_{\mu}\right)$}

The momentum coefficient is a non-dimensional parameter used to describe the mass flow rate of the air being ejected out of the slot of a $\mathrm{CC}$ system (which is fully defined in the subsequent Boundary Conditions section). The momentum coefficient used in this study was determined using guidance from past research. ${ }^{77}$ Experimental results on $\mathrm{CC}$ airfoils have described two different régimes of circulation control as a function of momentum coefficient, separation control and super circulation control. ${ }^{50,78,79}$ These two regimes exhibit different blowing efficiencies as defined by change in unit lift due to change in unit of momentum coefficient. ${ }^{80}$ As momentum coefficient is increased, separation control is defined as the initial entrainment of the separated flow behind the airfoil by the jet onto the Coanda surface. Once the jet separation point reaches the most aft surface of the Coanda surface and begins to penetrate the flow field below the airfoil, the separation control regime transitions into the super-circulation control regime. Figure 3-5 below differentiates the two different regimes of circulation control on a plot of $\mathrm{C}_{\mathrm{L}}$ vs. $C_{\mu} \cdot{ }^{50}$ 


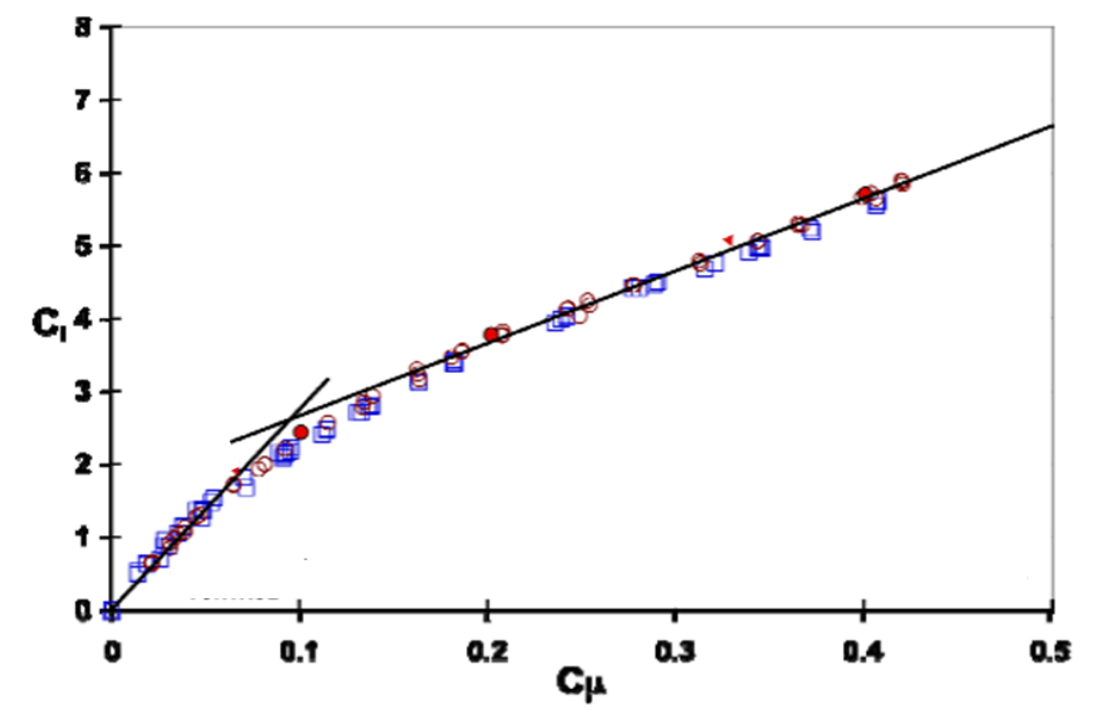

Figure 3-5: The two regions of circulation control as seen by experiment ${ }^{50}$

The momentum coefficient for this investigation was chosen such to provide adequate flow attachment for the various flap configurations tested at the different flight conditions, leading to the selection of a $\mathrm{C}_{\mu}$ value of 0.3 . By selecting a value well within the super-circulation regime, it ensured that each configuration would not experience jet separation prior to the end of the flap surface leading to a non-biased performance evaluation. Jet separation on the radial surfaces of a CC flap has also been shown to be difficult to predict using CFD. ${ }^{56-59,81-83}$

\section{Flap Placement/Integration}

To place the flap on the airfoil (Figure 3-6a.), several constraints were enforced to guarantee consistent flap location for the various flap configurations investigated. The first was to have a circular arc of radius $r_{1}$ intersect the upper and lower surface of the aft part of the airfoil, as pictured in Figure 3-6b. The upper intersection was then constrained to have the circular arc to become tangent with the upper surface of the 
airfoil (Figure 3-6c.). The circular arc of $r_{1}$ was then constrained to be perpendicular to the horizontal at the lower intersection of the airfoil (Figure 3-6d.), which finalized the flap's chord-wise placement on the airfoil. The second flap surface was then extended from the top end point of the first $r_{1}$ arc, being tangentially constrained to it (Figure 3-6e.). The second flap surface was then extended to the specified length, and ending on the airfoil's chord line (Figure 3-6f.). A spline was then added between the end of the second flap surface and the start point of the $r_{1}$ arc on the bottom of the airfoil, and constrained to be tangent to the existing airfoil surface at that location (Figure 3-6g.). Figure 3-6h. shows the completed flap integration for one of the analyzed flap configurations. Table 3-2 below also summarizes the flap placement/integration steps.

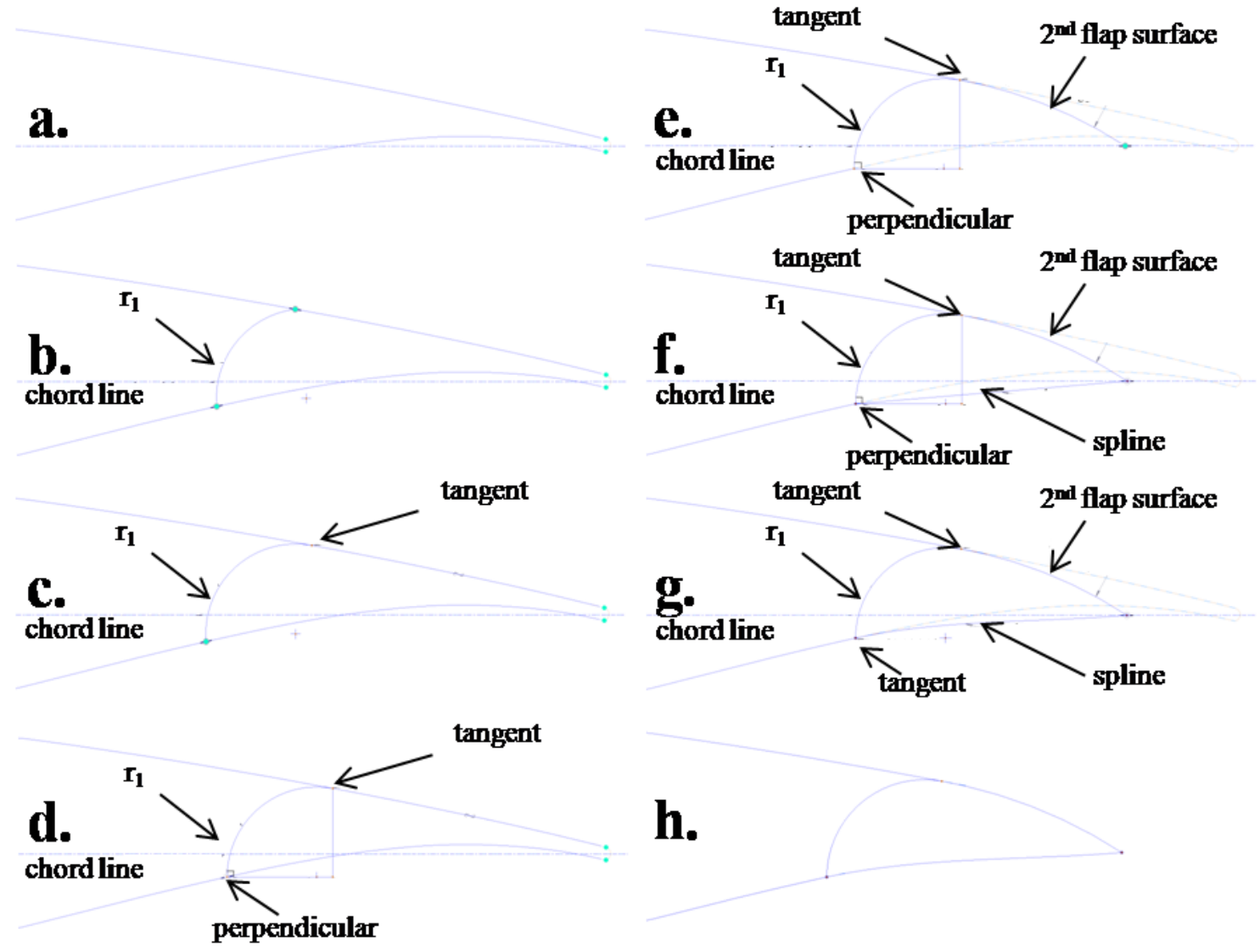

Figure 3-6: Flap placement and integration 
Table 3-2: Steps to place and integrate flap onto airfoil

\begin{tabular}{|c|c|c|}
\hline $\begin{array}{c}\text { Step } \\
\#\end{array}$ & Description & $\begin{array}{c}\text { Corresponding } \\
\text { Figure }\end{array}$ \\
\hline 1 & $\begin{array}{c}\text { Place circular arc of } r_{1} \text { in the aft region of airfoil, } \\
\text { intersecting upper and lower surfaces }\end{array}$ & Figure 3-6b. \\
\hline 2 & $\begin{array}{c}\text { Constrain } \mathrm{r}_{1} \text { to be tangent at intersection with the upper } \\
\text { surface of the airfoil }\end{array}$ & Figure 3-6c. \\
\hline 3 & $\begin{array}{c}\text { Constrain } \mathrm{r}_{1} \text { to be perpendicular with the horizontal at } \\
\text { intersection with the lower surface of the airfoil }\end{array}$ & Figure 3-6d. \\
\hline 4 & $\begin{array}{c}\text { Extend second flap surface from upper intersection of } \\
\mathrm{r}_{1} \text { and airfoil, making it tangent to } \mathrm{r}_{1}\end{array}$ & Figure 3-6e. \\
\hline 5 & $\begin{array}{c}\text { Extend second flap surface to specified length, and } \\
\text { have it end on the airfoil's chord line }\end{array}$ & Figure 3-6e. \\
\hline 6 & $\begin{array}{c}\text { Add spline between end of second flap surface and } \\
\text { intersection of } \mathrm{r}_{1} \text { and bottom of airfoil }\end{array}$ & Figure 3-6f. \\
\hline 7 & $\begin{array}{c}\text { Constrain spline to be tangent to bottom airfoil surface } \\
\text { at intersection of } \mathrm{r}_{1} \text { and bottom of airfoil }\end{array}$ & Figure 3-6g. \\
\hline
\end{tabular}

To create the trailing edge slot on the airfoil, a vertical element was added at the location of the flap's intersection of the upper surface of the airfoil. The element had a height of $\mathrm{h}_{\text {slot }}$ plus an additional $0.254 \mathrm{~mm}$, to account for the upper surface material thickness that was required for the manufacturing of the wind tunnel model. To keep the continuity of the upper airfoil surface with the slot and to ensure mechanical feasibility, a spline was added from 55\% chord of the airfoil to the slot location. This spline was constrained to be tangent to the airfoil surface at $55 \%$ chord and to be parallel to the tangent of the airfoil surface below the slot. Figure 3-7 depicts the modification of the airfoil to accommodate the trailing edge slot. Once this process of modifying the airfoil has taken place, the $\mathrm{CC}$ dual/prescribed radius flap will be placed and incorporated onto the airfoil of interest. 


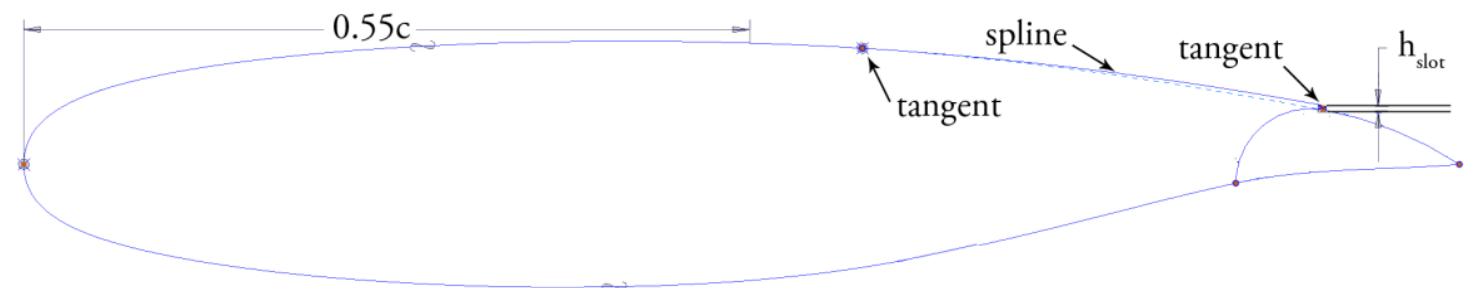

Figure 3-7: Trailing edge slot modification 


\section{Chapter 4: Parameter Studies}

Due to the emphasis on the geometrical design of the CC dual radius flap system, some of the parameters of the flap were held constant throughout the analysis. The first of which was the two-dimensional slot jet momentum coefficient $\left(\mathrm{C}_{\mu}\right)$, which is the driving parameter in the lift augmentation $\left(\Delta \mathrm{C}_{\mathrm{l}} / \mathrm{C}_{\mu}\right)$ of the $\mathrm{CC}$ operation. The other parameters that were held constant were the flap location (chord wise-direction), slot location, slot height $\left(\mathrm{h}_{\text {slot }}\right)$, the plenum shape, and the first radial surface of the flap $\left(\mathrm{r}_{1}\right)$. Holding these parameters constant isolated the flap surface aft of the $r_{1}$ arc (highlighted in Figure 4-1) to be the only influence on aerodynamic changes in the investigation.

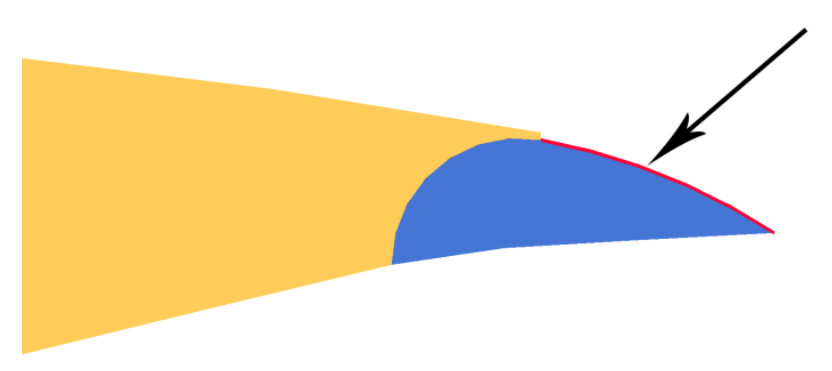

Figure 4-1: Aft segment of flap surface

\section{Dual Radius}

The parameters of interest in this research were initially the second radius $\left(\mathrm{r}_{2}\right)$ and the length of the flap for strictly dual radius flap configurations. The length of the dual radius flap had a direct influence on the departure angle $(\theta)$ of the upper surface due to the previous constraints of placing the flap on the airfoil. By changing the length of the flap, which directly shifted $\theta$, it influenced the turning angle of the flow at a set flap deflection. By having a shorter flap with a greater $\theta$, it was predicted that the flow would 
be subject to more turning by the decreased radius of the curved surface but also have more difficulty staying attached as the flow proceeds along the flap and loses momentum. This shorter flap, with larger $\theta$, would be beneficial to the aircraft's performance during takeoff and landing operations by providing a large amount of lift-augmentation, but would also cause significant pressure drag during cruise due to its blunt trailing edge.

The second parameter that was investigated in this preliminary study on the dual radius configuration was $r_{2}$ and the resulting ratio of $r_{2} / r_{1}$. Having small $r_{2}$ values, similar to $r_{1}$, was predicted to result in increased flow turning angles and yield shorter flap lengths. Although the shorter and steeper flap may encounter flow separation, as mentioned above, the less drastic change in radii on the flap surface could possibly facilitate flow attachment by encouraging a more favorable pressure gradient in this gradually changing region. With $r_{2}$ values significantly larger than $r_{1}$, the flow would not have as much difficulty staying attached to the contour of the flap but may encounter issues with the larger discontinuity in the radius of curvature when $r_{1}$ changes to $r_{2}$. This larger $r_{2}$ would also not be as effective in lift augmentation by not achieving large $\theta$ values at the trailing edge of the flap surface.

The results of this preliminary study on dual radius flaps, which was performed under a NASA research contract, ${ }^{7}$ provided insight into what additional flap parameters could be investigated to further characterize the geometry of the CC flap. This analysis was carried out using two-dimensional CFD with the realizable k- $\varepsilon$ turbulence model. ${ }^{84}$ The tested geometries, with their respective specifications are presented blow in Figure 4-2. The study showed that the shorter dual radius flap lengths with larger $\theta$ values augmented greater lift coefficients, but the flaps with larger $r_{2} / r_{1}$ ratios had better L/D 
characteristics. Though the flaps with larger $r_{2} / r_{1}$ ratios produced an increase in $L / D$, as the length of the same flap is increased, the favorable L/D values quickly diminish. Unfortunately, detailed investigation of the pressure and skin friction distribution on these airfoils was not performed to further analyze the trends.

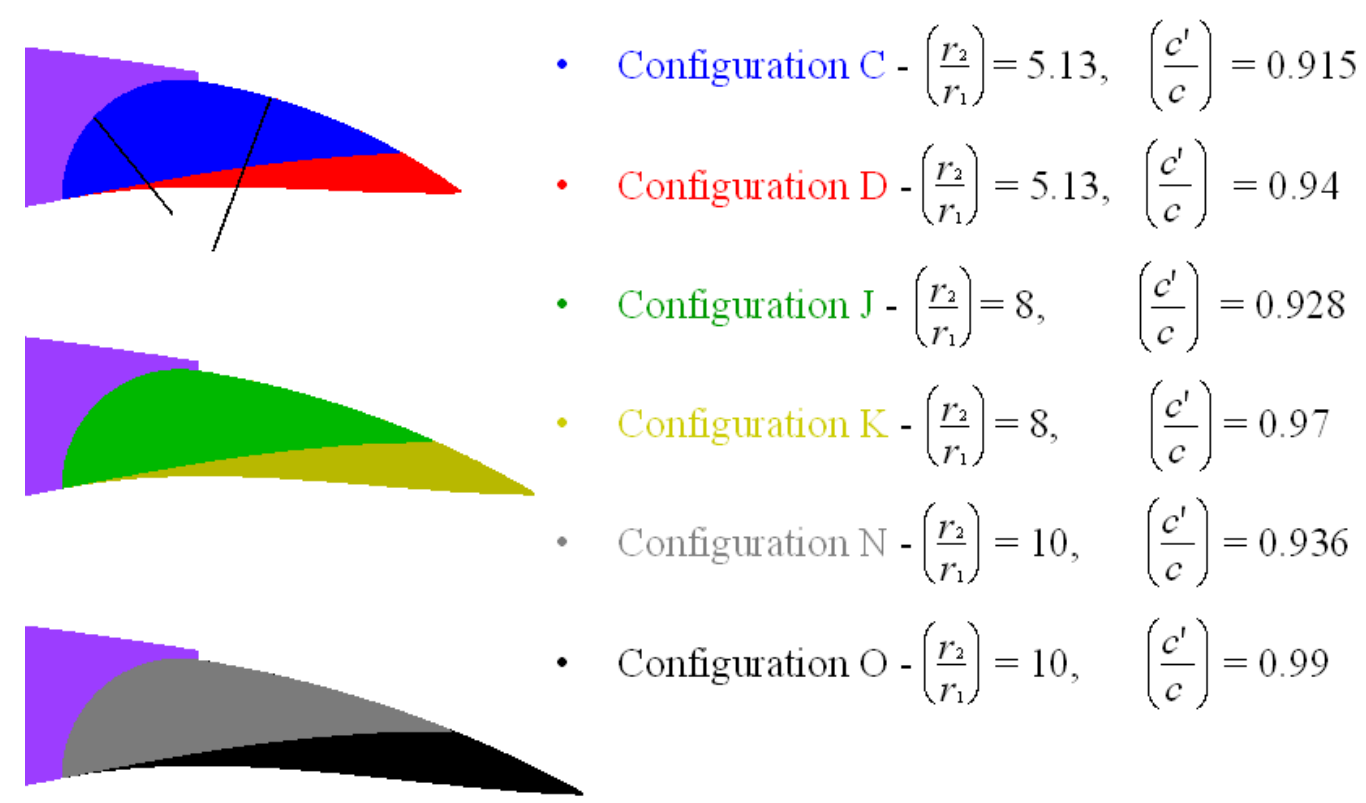

Figure 4-2: Previously studied CC dual radius flap configurations

\section{Prescribed Radius}

Additional research was performed on the geometric specifications of the flap surface, given the preliminary results, to further investigate the flap shape. The parameters that were examined were the shape, slope, rate of change of the slope, and curvature of the flap surface. The goal of this study was to analyze the geometric properties of the dual radius and develop alternative surfaces that would have the potential of offering enhanced aerodynamics. 
The alternate curve that was compared to the constant radius curves of the dual radius flap was designated as a prescribed radius curve. The prescribed radius curve takes on a user-specified radius given the position along the chord-wise direction. This new curve was intended to be similar to a spiral, having a constantly increasing radius which has been seen to experience self-preserving boundary layer characteristics along its surface. ${ }^{71}$ The prescribed radius curve's spiral-like nature give it gradual change in radius of curvature along the flap surface, rather than the instantaneous change of radii of the dual radius flap.

The beginning of the prescribed radius curve was set to match the location, slope, and slope rate of change of the end of the first constant radius surface of $r_{1}$. By matching the slope rate of change it allowed for the flap surface to have a continuous curvature between $r_{1}$ and the prescribed radius curve, along with having the same slope. From this location, the prescribed radius was chosen to have a cubic relationship with respect to the chord-wise direction of the airfoil to allow for two other constraints to be assigned to the curve on top of the constraints that were already set by the first $r_{1}$ curve. These two constraints were the selection of end location and end radius of the prescribed radius curve. The first additional constraint permitted the matching of curve length between the dual radius and prescribed radius surfaces. The end radius of curvature of the prescribed radius surface was set to zero, assisting the flow on the upper surface to leave the trailing edge of the flap/airfoil with no angular component, increasing Kutta condition. These flap design parameters defined the next set of CC flap configurations that were investigated. 


\section{Curve Comparison}

For the analytic comparison of the two curves, a common dual radius shape, ${ }^{1,2,5,15,49,52,53}$ with a $\mathrm{r}_{2} / \mathrm{r}_{1}$ ratio of 5 , was evaluated against a prescribed radius with the same flap chord length and similar shape. This corresponded with the prescribed radius flap having an $r_{2 \_a v g} / r_{1}$ of 30.7 and an end radius $60 * r_{1}$. The results are presented below in Figure 4-3.

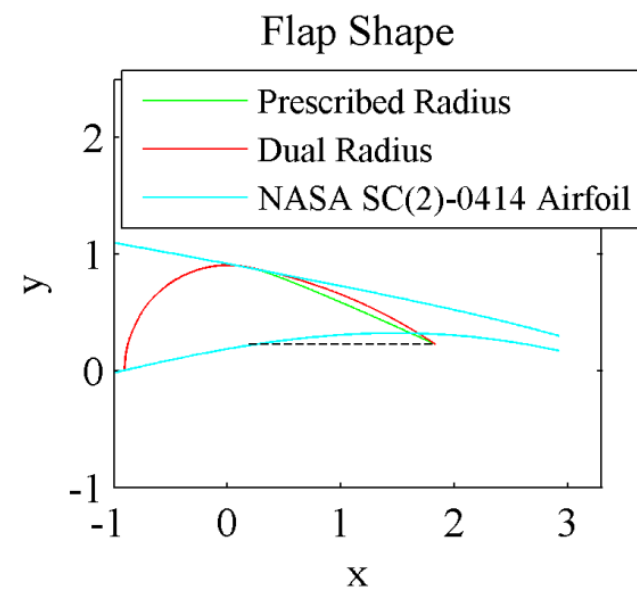

Flap Slope Rate of Change

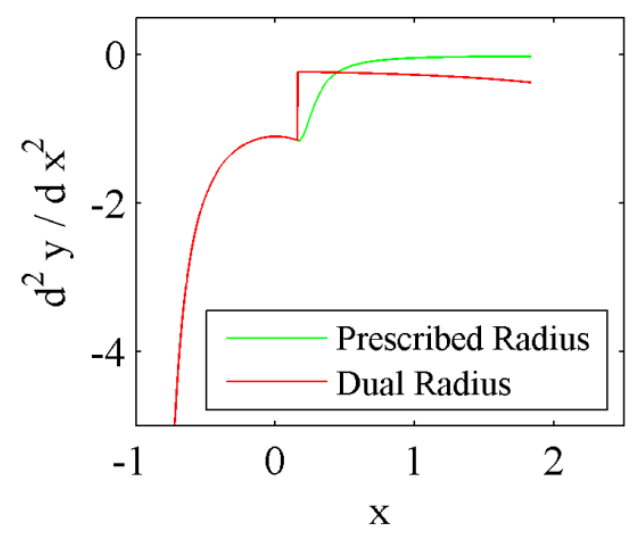

Flap Slope

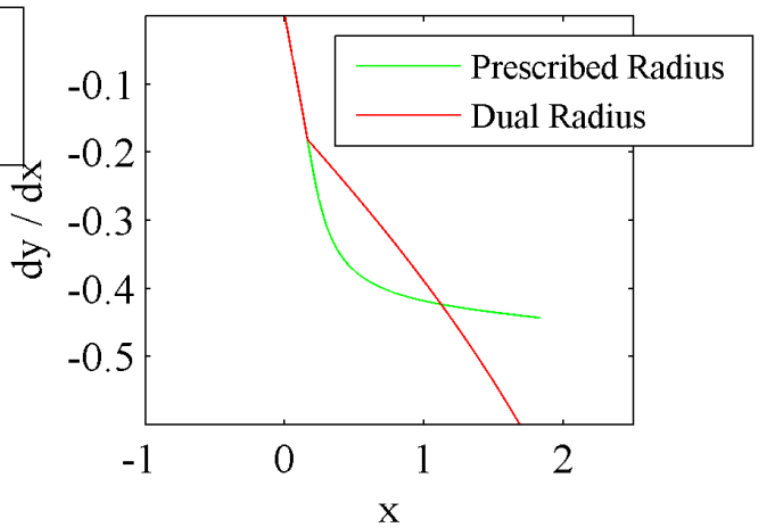

Flap Curvature

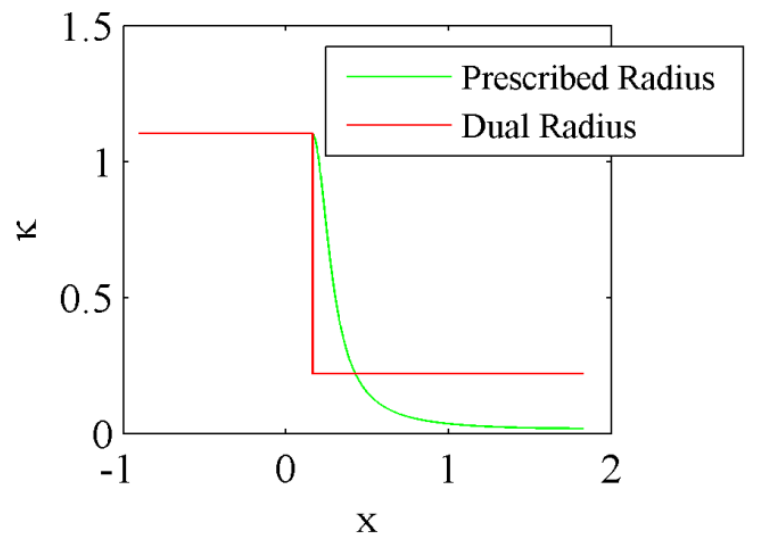

Figure 4-3: Flap surface analysis

From the flap shape plot, the two curves appear very similar in physical appearance with the prescribed radius curve dipping slightly under the dual radius curve. Though, the rest of the four plots show the analytical difference between the curves. The 
flap slope plot shows the smooth transition from the $\mathrm{r}_{1}$ curve to the secondary curve for the prescribed radius, where the dual radius has a non-smooth change in slope even though the flap slope is continuous. This can be seen further in the flap slope rate of change plot, where the dual radius has a discontinuity between $r_{1}$ and $r_{2}$. Although the prescribed radius curve experiences a steep slope after the $r_{1}$ curve on the curvature plot, it keeps a smooth slope transition between the two curves. Figure 4-4 below displays a close up of the transition, where the smoothness can be seen more clearly on the prescribed radius curve. This smooth transition in curvature from $r_{1}$ to a prescribed variation in radius is believed to encourage flow attachment as the jet flow travels over the flap surface, thus being a central component in the following analysis.

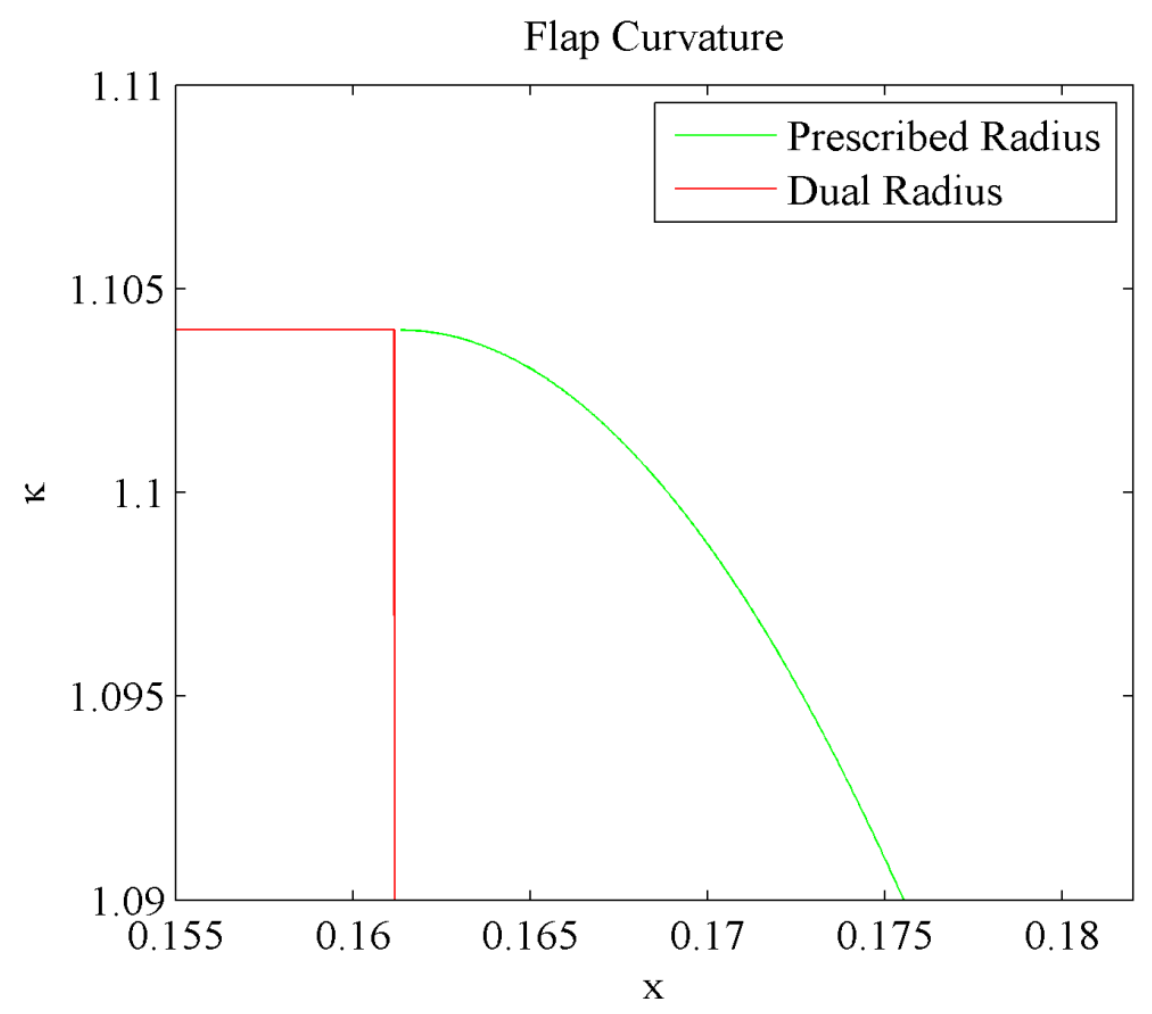

Figure 4-4: Curvature of flap surfaces 


\section{Testing Configurations}

Using analytical analysis of the curves, along with preliminary results, ${ }^{85}$ six geometries were created to further investigate and are presented in Figure 4-5 with their respective specifications in Table 4-1. The callout names of each represent either dual radius $(\mathrm{DR})$ or prescribed radius $(\mathrm{PR})$, and the percentage of flap chord $\left(\mathrm{c}_{\mathrm{f}}\right)$ to flapmodified chord $\left(c^{\prime}\right)$. The driving parameters of the dual radius flaps were $r_{2}$ and the length of the flap, where the prescribed radius flaps were driven by $r_{2 \_a v g}$ and length due to the cubic variation in radius. DR16 was the baseline dual radius configuration, being a common configuration of CC flap systems and having previous experimental ${ }^{1,2,5,15,49,52}$ and numerical data available. ${ }^{52,53}$ PR16 was based on the same length as DR16, with the smooth slope and curvature transitions accomplished by the prescribed radius. DR22 and PR22 were created by extending the length of the flap and increasing $r_{2}$ or $r_{2 \_a v g}$ to have the upper surface of the flap to closely match the top of the original airfoil. This effort was made to keep the flap-modified airfoil as close as possible to the original airfoil's shape. DR19 and PR19 where then developed to add an intermediate flap between the 16 and -22 series, resulting in a length and radius in between the two.

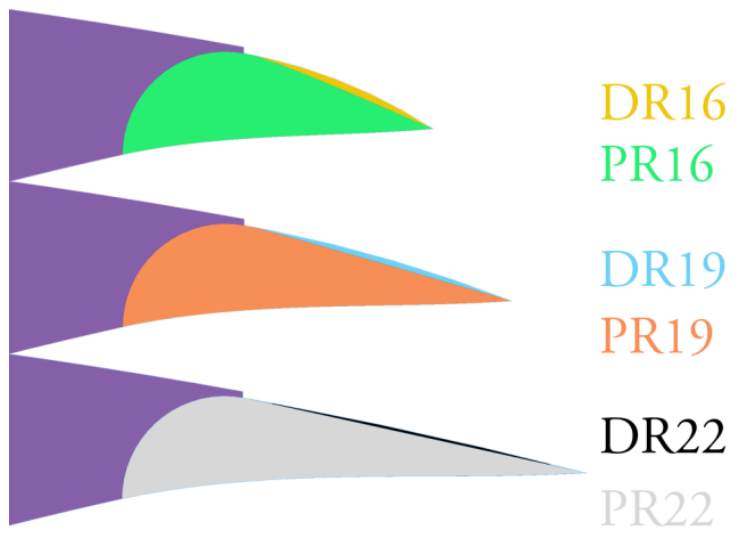

Figure 4-5: Examined flap configurations 
Table 4-1: Flap configuration specifications

\begin{tabular}{|c|c|c|c|c|}
\hline Configuration & $\mathbf{c}^{\prime} / \mathbf{c}$ & $\mathbf{c}_{\mathbf{f}} / \mathbf{c}^{\prime}$ & $\left(\mathbf{r}_{\mathbf{2}}\right.$ or $\mathbf{r}_{\mathbf{2}}$ avg $) / \mathbf{r}_{\mathbf{1}}$ & $\boldsymbol{\theta}$ \\
\hline DR16 & 0.9414 & 0.1558 & 5 & $33.12^{\circ}$ \\
\hline PR16 & 0.9414 & 0.1558 & 30.7 & $23.92^{\circ}$ \\
\hline DR19 & 0.9782 & 0.1875 & 14.14 & $21.22^{\circ}$ \\
\hline PR19 & 0.9782 & 0.1875 & 291.5 & $16.18^{\circ}$ \\
\hline DR22 & 1.015 & 0.2169 & 48 & $14.36^{\circ}$ \\
\hline PR22 & 1.015 & 0.2170 & 3721 & $12.35^{\circ}$ \\
\hline
\end{tabular}

All flap configurations were analyzed at three different flap settings which were takeoff, approach/landing, and cruise. The takeoff flap setting was chosen to be a $60^{\circ}$ flap deflection, due to the large lift augmentation encountered with moderate drag penalties to follow. ${ }^{2}$ The approach/landing flap was set at a $90^{\circ}$ deflection, in order to achieve high lift along with high drag values. For the cruise setting, the flap deflection was set to $0^{\circ}$ and the CC slot on the upper surface of the airfoil was retracted to reduce drag. The three different flap settings are displayed below in Figure 4-6 for the PR19 configuration. 


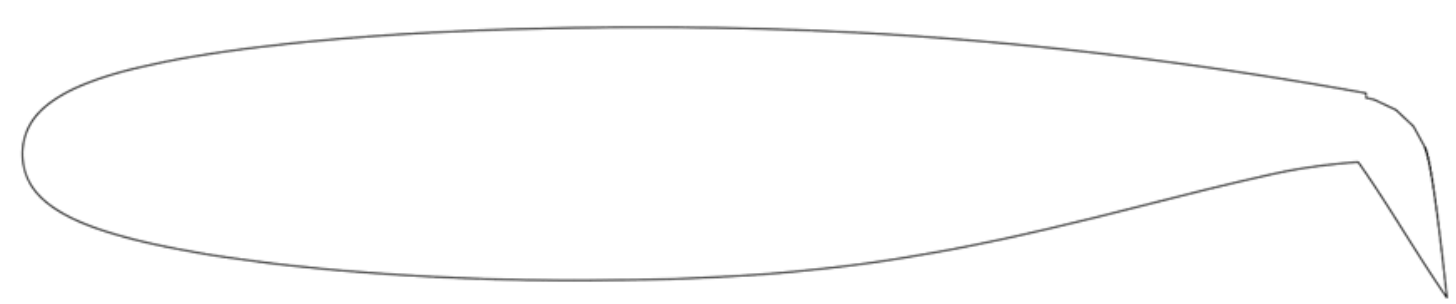

Takeoff - $60^{\circ}$ Flap Deflection (Slot Open)

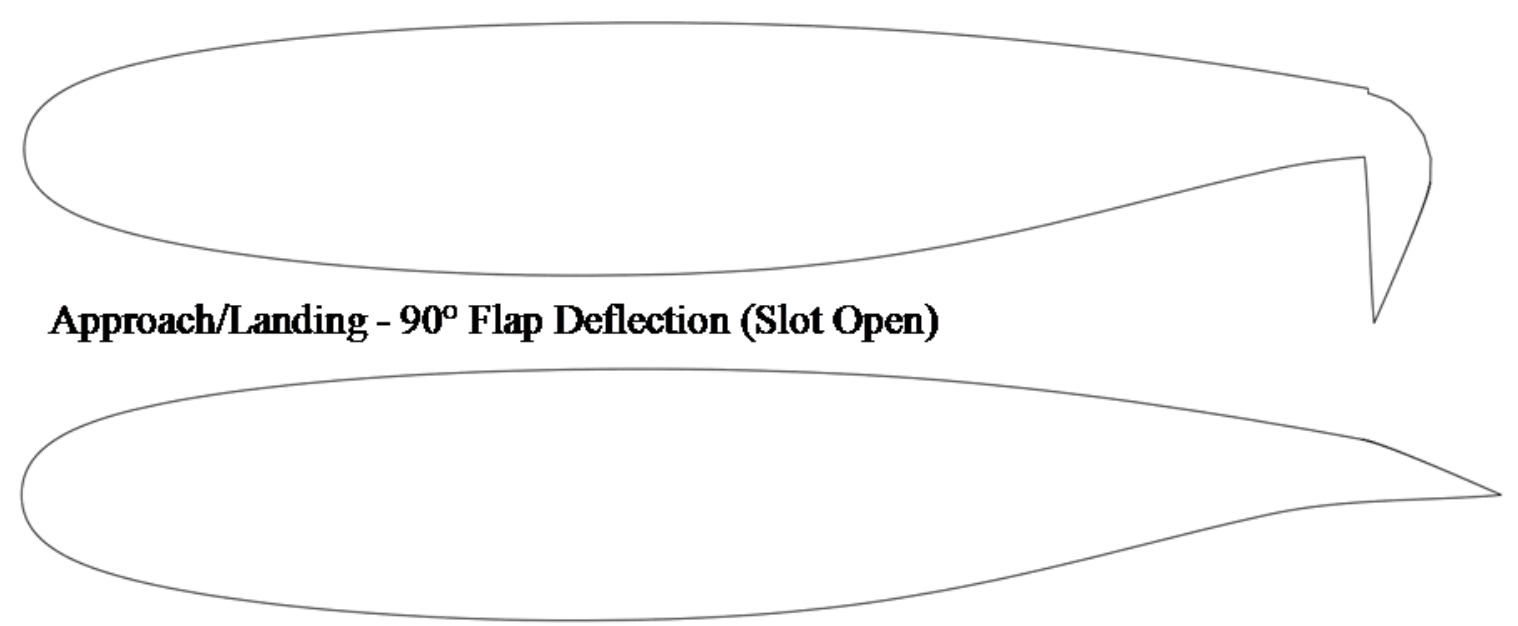

Cruise - $0^{\circ}$ Flap Deflection (Slot Closed)

Figure 4-6: 3 flap settings analyzed 


\section{Chapter 5: Computational Analysis Architecture}

\section{Governing Equations}

The Reynolds Averaged Navier-Stokes (RANS) equations were used in the subsequent simulations to describe the fluid motion. With the RANS formulations separating the time-dependent turbulent velocity fluctuations from the mean flow velocity, the small scale and high-frequency effects of turbulent flows are removed. Though, in order to produce a closed system of solvable governing equations, the RANS equations require a turbulence model to address the introduction of a set of unknowns which are functions of the velocity fluctuations called the Reynolds stresses.

The ensuing set of governing equations follows the derivations presented by Tannehill, Anderson, and Pletcher ${ }^{86}$ and is presented with mass-weighted averaged variables, being more convenient for compressible flows. Starting with the conservation of mass, the continuity equation can be written in Reynolds form as

$$
\frac{\partial \bar{\rho}}{\partial t}+\frac{\partial}{\partial x_{j}}\left(\bar{\rho} \tilde{u}_{j}\right)=0
$$

which is valid for both incompressible and compressible flows.

The conservation of momentum is represented by the following RANS form of the momentum equation 


$$
\frac{\partial}{\partial t}\left(\bar{\rho} \tilde{u}_{i}\right)+\frac{\partial}{\partial x_{j}}\left(\bar{\rho} \tilde{u}_{i} \tilde{u}_{j}\right)=-\frac{\partial \bar{p}}{\partial x_{i}}+\frac{\partial}{\partial x_{j}}\left(\bar{\tau}_{i j}+R_{i j}\right)
$$

when neglecting viscosity fluctuations and doubly primed viscous terms, the viscous stress tensor $\bar{\tau}_{i j}$ becomes

$$
\bar{\tau}_{i j}=\mu\left[\left(\frac{\partial \tilde{u}_{i}}{\partial x_{j}}+\frac{\partial \tilde{u}_{j}}{\partial x_{i}}\right)-\frac{2}{3} \delta_{i j} \frac{\partial \tilde{u}_{k}}{\partial x_{k}}\right]
$$

and $\mathrm{R}_{\mathrm{ij}}$ represents the Reynolds stress tensor, which is defined as

$$
R_{i j}=-\overline{\rho u_{i}^{\prime \prime} u_{j}^{\prime \prime}}
$$

The Reynolds averaged form of the energy equation can be written

$$
\frac{\partial}{\partial t}(\bar{\rho} \tilde{H})+\frac{\partial}{\partial x_{j}}\left(\bar{\rho} \tilde{u}_{j} \tilde{H}+\overline{\rho u_{j}^{\prime \prime} H^{\prime \prime}}-k_{T} \frac{\partial \bar{T}}{\partial x_{j}}\right)=\frac{\partial \bar{p}}{\partial t}+\frac{\partial}{\partial x_{j}}\left(\tilde{u}_{i} \bar{\tau}_{i j}+\overline{u_{i}^{\prime \prime} \tau_{i j}}\right)
$$

where $\mathrm{k}_{\mathrm{T}}$ is thermal conductivity, and is described as

$$
k_{T}=\frac{c_{p}}{\operatorname{Pr}} \mu
$$

and $\operatorname{Pr}=0.72$ for air at standard conditions.

In order to achieve closure on the RANS governing equations it is necessary to make assumptions about the apparent Reynolds stress and heat flux quantities. One of the assumptions that the chosen turbulence model uses is the Boussinesq approximation, which relates the turbulent shearing stresses to the rate of mean strain through an 
apparent "eddy" viscosity. The Boussinesq approximation defines the general Reynolds stress tensor as

$$
R_{i j}=2 \mu_{T} S_{i j}-\frac{2}{3} \delta_{i j}\left(\mu_{T} \frac{\partial u_{k}}{\partial x_{k}}+\rho k\right)
$$

where $\mu_{\mathrm{T}}$ is the turbulent viscosity, $k$ is the kinetic energy of turbulence where

$$
k=\frac{\overline{u_{i}^{\prime} u_{i}^{\prime}}}{2}
$$

and $\mathrm{S}_{\mathrm{ij}}$ is the rate of the mean strain tensor which is defined as

$$
S_{i j}=\frac{1}{2}\left(\frac{\partial u_{i}}{\partial x_{j}}+\frac{\partial u_{j}}{\partial x_{i}}\right)
$$

The law of the wall states that the average velocity of a turbulent flow is proportional to the logarithm of the distance from the location of interest to the wall or boundary of the fluid region (von Karman). ${ }^{87}$ This law is applicable to regions of the flow that are near the boundary, typically $<20 \%$ of the height of the flow, and for flows at high Reynolds numbers. The law of the wall relates the dimensionless velocity of the fluid to the dimensionless distance from the boundary with

$$
u^{+}=\frac{1}{\kappa^{\prime}} \ln y^{+}+C^{+}
$$

where $\kappa^{\prime}$ is the von Karman constant, $\mathrm{C}^{+}$is a dimensionless constant, and $\mathrm{y}^{+}$is the dimensionless distance from the boundary and is defined as 


$$
y^{+}=\frac{y u_{\tau}}{v}
$$

where $\mathrm{y}$ is the distance to the wall/boundary, $v$ is the kinematic viscosity, and $\mathrm{u}_{\tau}$ is the friction velocity. The friction velocity is expressed as

$$
u_{\tau}=\sqrt{\frac{\tau_{w}}{\rho}}
$$

where $\tau_{\mathrm{w}}$ is the wall shear stress and $\rho$ is the fluid density. And the definition of the dimensionless velocity is given as

$$
u^{+}=\frac{u}{u_{\tau}}
$$

where $\mathrm{u}$ is the velocity of the fluid. From experiments the von Karman constant is found to be $\kappa^{\prime} \approx 0.41$ for both smooth and rough surfaces and the dimensionless constant $\mathrm{C}^{+} \approx$ 5.0 for smooth surfaces. ${ }^{88}$

As the fluid approaches the boundary the law of the wall begins to break down and over predict the mean velocity of the flow. This occurs within the buffer or transition layer $\left(3.5<\mathrm{y}^{+}<30\right)$ and viscous sublayer $\left(\mathrm{y}^{+}<3.5\right)$. Due to this omission of the law of the wall the computational mesh used to analyze the flow must contain cells within the viscous sublayer to appropriately capture the viscous effects of the fluid (typical $\mathrm{y}^{+}$target of < 1). A standard velocity profile of the mean velocity of a turbulent boundary layer is displayed below in Figure 5-1, which also presents the law of the wall approximation with the dashed line. ${ }^{89}$ 


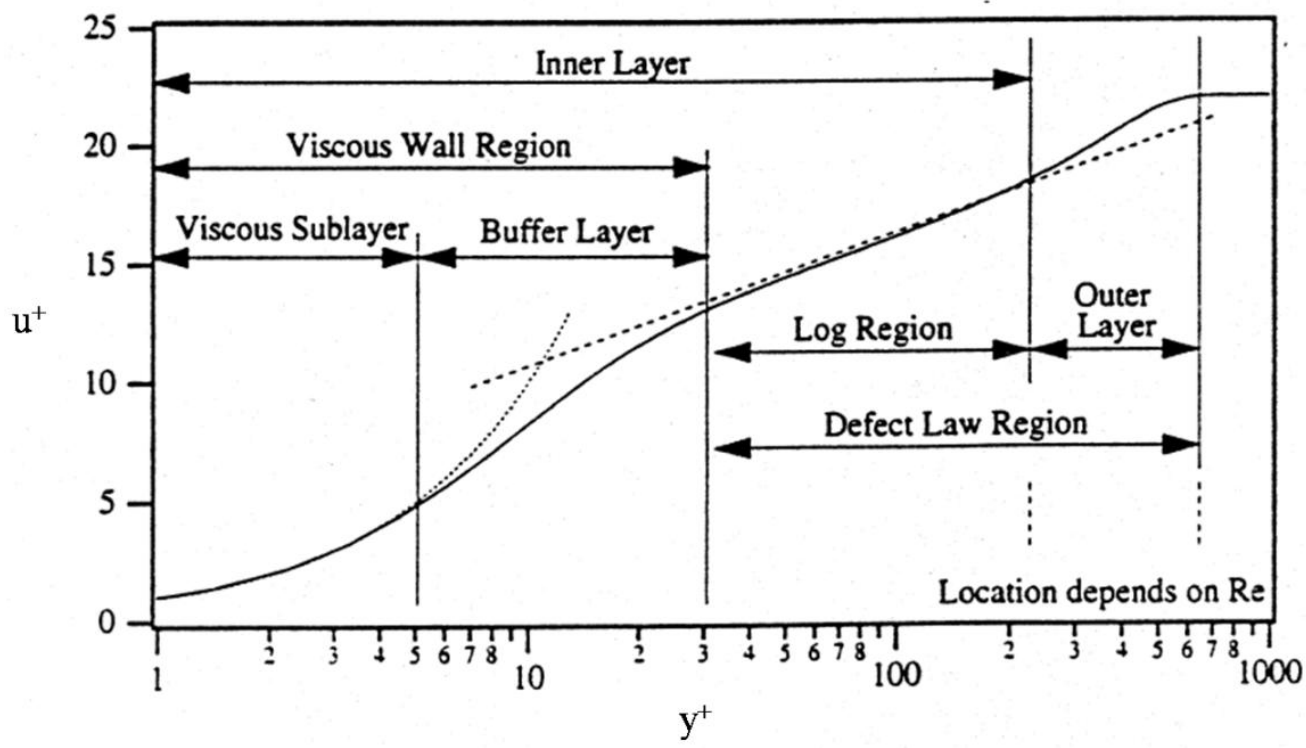

Figure 5-1: A standard velocity profile for a turbulent boundary layer ${ }^{89}$

\section{Turbulence Model Selection}

The turbulence model selected for this simulation was the two-equation realizable $\mathrm{k}-\varepsilon .{ }^{84}$ The selection of a two-equation turbulence model allowed for the turbulent velocity and length scale to be decoupled, letting the upstream flow have influence on the length scale. Since the first use of k- $\varepsilon$ turbulence models by Harlow and Nakayama ${ }^{90}$ several improvements have been made by researchers, including the work of Launder and Spalding, ${ }^{91}$ Yakhot and Orszag, ${ }^{92}$ and Shih, Liou, Shabbir, Yang, and Zhu. ${ }^{84}$ The main advantages of the improved realizable k- $\varepsilon$ model over the standard model are a new transport equation for the turbulent dissipation rate and a new representation of a critical coefficient of the model, $\mathrm{C}_{\mu \mathrm{T}}$. The new representation of $\mathrm{C}_{\mu \mathrm{T}}$ expresses the coefficient as a function of mean flow and turbulence properties, where the standard model assumed it to be constant. This allows the model to better predict the normal stresses of the turbulent flow. 
The following equations are taken from Reference 93. The two transport equations that are used in the realizable k- $\varepsilon$ turbulence model describe the turbulent kinetic energy and dissipation rate of the fluid. These equations can be written

$$
\begin{gathered}
\frac{\partial}{\partial t}(\rho k)+\frac{\partial}{\partial x_{j}}\left(\rho k u_{j}\right)=\frac{\partial}{\partial x_{j}}\left[\left(\mu+\frac{\mu_{t}}{\sigma_{k}}\right) \frac{\partial k}{\partial x_{j}}\right]+P_{k}+P_{b}-\rho \varepsilon-Y_{M}+S_{k} \\
\frac{\partial}{\partial t}(\rho \varepsilon)+\frac{\partial}{\partial x_{j}}\left(\rho u_{j}\right)=\frac{\partial}{\partial x_{j}}\left[\left(\mu+\frac{\mu_{t}}{\sigma_{\varepsilon}}\right) \frac{\partial \varepsilon}{\partial x_{j}}\right]+\rho C_{1} S_{\varepsilon}-\rho C_{2} \frac{\varepsilon^{2}}{k+\sqrt{v \varepsilon}}+C_{1 \varepsilon} \frac{\varepsilon}{k} C_{3 \varepsilon} P_{b}+S_{\varepsilon}
\end{gathered}
$$

where

$$
\begin{gathered}
C_{1}=\max \left[0.43, \frac{\eta}{\eta+5}\right] \\
\eta=S \frac{k}{\varepsilon}
\end{gathered}
$$

$\mathrm{C}_{2}, \mathrm{C}_{1 \varepsilon}$, and $\mathrm{C}_{3 \varepsilon}$ are model constants. $\sigma_{\mathrm{k}}$ and $\sigma_{\varepsilon}$ are turbulent Prandtl numbers and $\mathrm{S}_{\mathrm{k}}$ and $\mathrm{S}_{\varepsilon}$ are user-defined source terms, each for $\mathrm{k}$ and $\varepsilon$ respectively. $\mathrm{Y}_{\mathrm{M}}$ is a compressibility factor. $\mathrm{P}_{\mathrm{k}}$ is the generation of turbulent kinetic energy due to mean velocity gradients, which is given as

$$
P_{k}=-\overline{u_{i}^{\prime} u_{j}^{\prime}} \frac{\partial u_{j}}{\partial x_{i}}
$$

or

$$
P_{k}=\mu_{t} S^{2}
$$


where $\mathrm{S}$ is the modulus of the mean rate-of-strain tensor, defined as

$$
S=\sqrt{2 S_{i j} S_{i j}}
$$

$P_{b}$ in equation (14) and (15) above represents the generation of turbulent kinetic energy due to buoyancy and can be written as

$$
P_{b}=\beta g_{i} \frac{\mu_{t}}{\operatorname{Pr}_{t}} \frac{\partial T}{\partial x_{i}}
$$

where $\operatorname{Pr}_{t}$ is the turbulent Prandtl number, which is defaulted to the value of 0.85 for both standard and realizable $\mathrm{k}-\varepsilon$ models. $\quad \beta$ is the coefficient of thermal expansion, and is given

$$
\beta=-\frac{1}{\rho}\left(\frac{\partial \rho}{\partial T}\right)_{p}
$$

The turbulent viscosity term, $\mu_{t}$, from equation (21) is modeled by the definition

$$
\mu_{t}=\rho C_{\mu T} \frac{k^{2}}{\varepsilon}
$$

where the realizable k- $\varepsilon$ model defines $C_{\mu \mathrm{T}}$ as

$$
C_{\mu T}=\frac{1}{A_{0}+A_{s} \frac{k U^{*}}{\varepsilon}}
$$


where

$$
\begin{gathered}
U^{*} \equiv \sqrt{S_{i j} S_{i j}+\widetilde{\Omega}_{i j} \tilde{\Omega}_{i j}} \\
\tilde{\Omega}_{i j}=\Omega_{i j}-2 \varepsilon_{i j k} \omega_{k} \\
\Omega_{i j}=\overline{\Omega_{i j}}-\varepsilon_{i j k} \omega_{k}
\end{gathered}
$$

$\overline{\Omega_{i j}}$ is the mean rate-of-rotation tensor that is referenced to a rotating reference frame with an angular velocity of $\omega_{\mathrm{k}} . \mathrm{A}_{0}$ and $\mathrm{A}_{\mathrm{s}}$ are model constants.

The realizable k- $\varepsilon$ turbulence model was chosen over the one-equation SpalartAllmaras ${ }^{57,61,94}$ and two-equation k- $\omega^{95}$ models because of its ability to capture features of turbulence with a high quality near-wall mesh, ${ }^{55}$ and being commonly used and robust. ${ }^{51}$ Although one- and two-equation turbulence models have been shown to typically overpredict the lift coefficients of airfoils in CC flows, ${ }^{50,52-59,61,80,81,83}$ the chosen model has shown benefits over other models in parametric studies and has been proven to consistently depict aerodynamic trends of CC flow features. ${ }^{96}$ The over-prediction of lift is caused by over-predicting turbulent kinetic energy ${ }^{56}$ and failure to follow the streamline turning of $\mathrm{CC}$ flows ${ }^{7}$ which make these models not adequate for capturing separation or recirculation regions. The four-equation nonlinear $v^{2}-f$ turbulence model with curvature correction has shown more promise in accurately predicting all regions of $\mathrm{CC}$ flow, ${ }^{59,83}$ but is computationally expensive and less robust. Since the extent of this research was to observe the performance characteristics of the different geometrical flap parameters rather than validation of experimental data or simulating stall, the fast and 
robust realizable $\mathrm{k}-\varepsilon$ model was accepted as the turbulence model of choice for this research.

\section{Solver Selection/Settings}

The solver used for the simulation is the RANS computational fluid dynamics (CFD) solver Fluent 6.3. ${ }^{97}$ Fluent was set to implicit and density-based. A pressure farfield was the boundary type assigned to the inlet, outlet, and symmetry planes. The airfoil and plenum wall were treated as walls, with the plenum inlet assigned to a pressure inlet boundary type. No near-wall treatment was applied to the selected turbulence model due to the grid refinement, discussed further in the following section.

Table 5-1 below displays a summary of the solver settings that were used for the following analysis in Fluent.

Table 5-1: Fluent solver settings

\begin{tabular}{|c|c|}
\hline Solver & Density-Based/Compressible \\
\hline Turbulence Model & Realizable k- $\varepsilon$ \\
\hline Near-Wall Treatment & None \\
\hline Density Calculator & Ideal-Gas Law \\
\hline Viscosity Calculator & Sutherland's Law \\
\hline Domain Boundary Type & Pressure Far-Field \\
\hline Plenum Inlet Boundary Type & Pressure Inlet \\
\hline
\end{tabular}

\section{Computational Mesh}

To accurately capture the complex flow physics of circulation control, a semistructured mesh was utilized. This allowed for different meshing schemes in the various regions that made up the entire computational domain, which consisted of a structured exterior, an unstructured interior region, and a structured slot, boundary layer, and wake 
region. Figure 5-2 below displays the entire domain used for the simulations. This figure shows the details of the structured exterior region which promote cell orientation in the freestream direction, helping in reducing the time for the solver to compute the governing equations. This exterior region extends five chord-lengths above and forward of the airfoil, ten chord-lengths downward, and fifteen chord-lengths aft. Having this large domain, ensured that the large turning of the flow and high mixing occurring in the wake, that are present during CC operation, were captured.

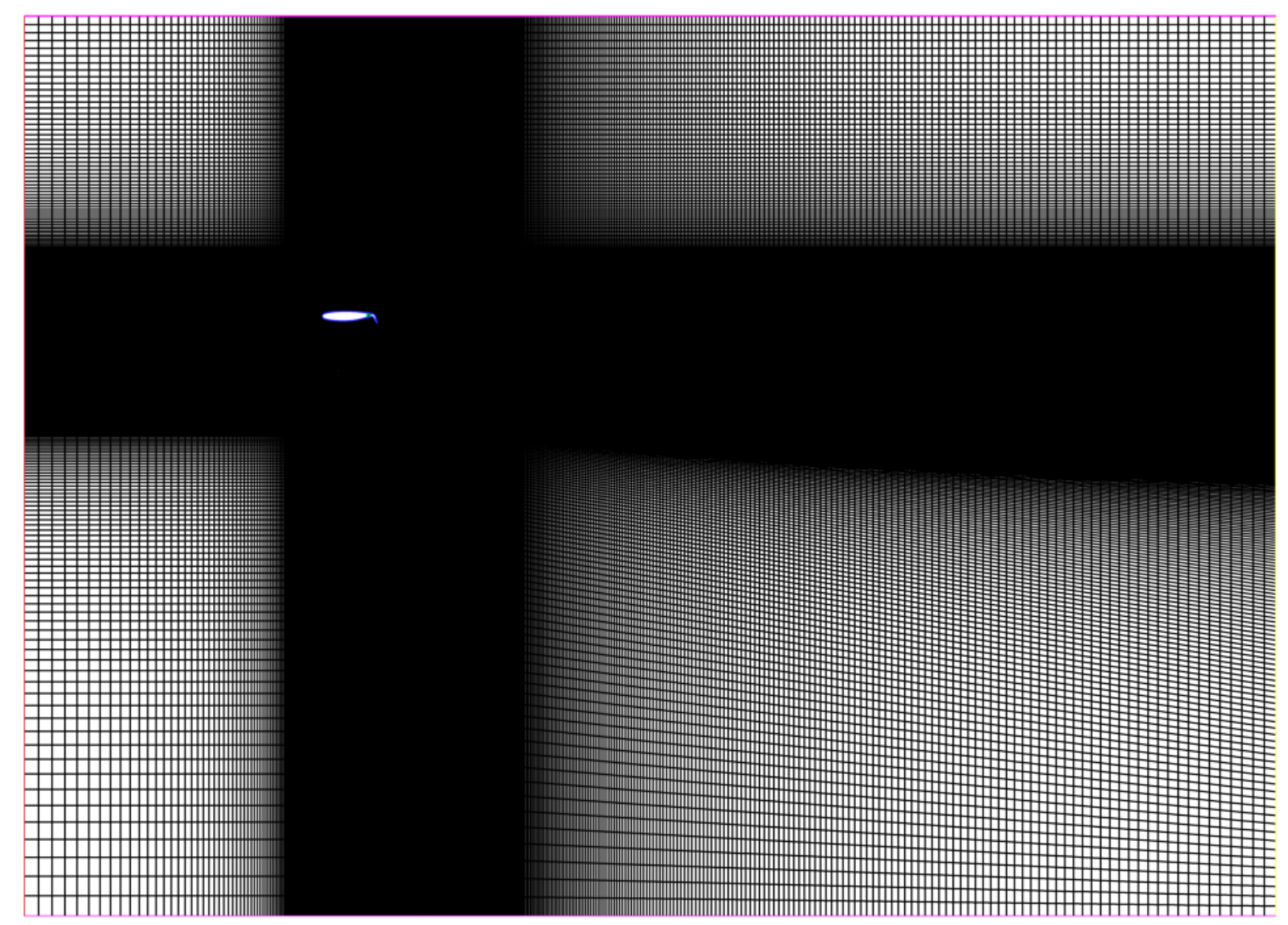

Figure 5-2: Entire computational domain

The interior region of the domain was left unstructured to help in the transition from the highly curved structured grid of the airfoil, flap, and wake to the orthogonal 
structured grid of the exterior region. This region is depicted below in Figure 5-3. The interior region was extended a half of a chord-length forward and above the airfoil and a chord-length and a half below and aft of the airfoil. The unstructured grid also allowed for a more rapid growth in cells in regions that did not need the refinement, promoting a lower cell density where it was not needed.

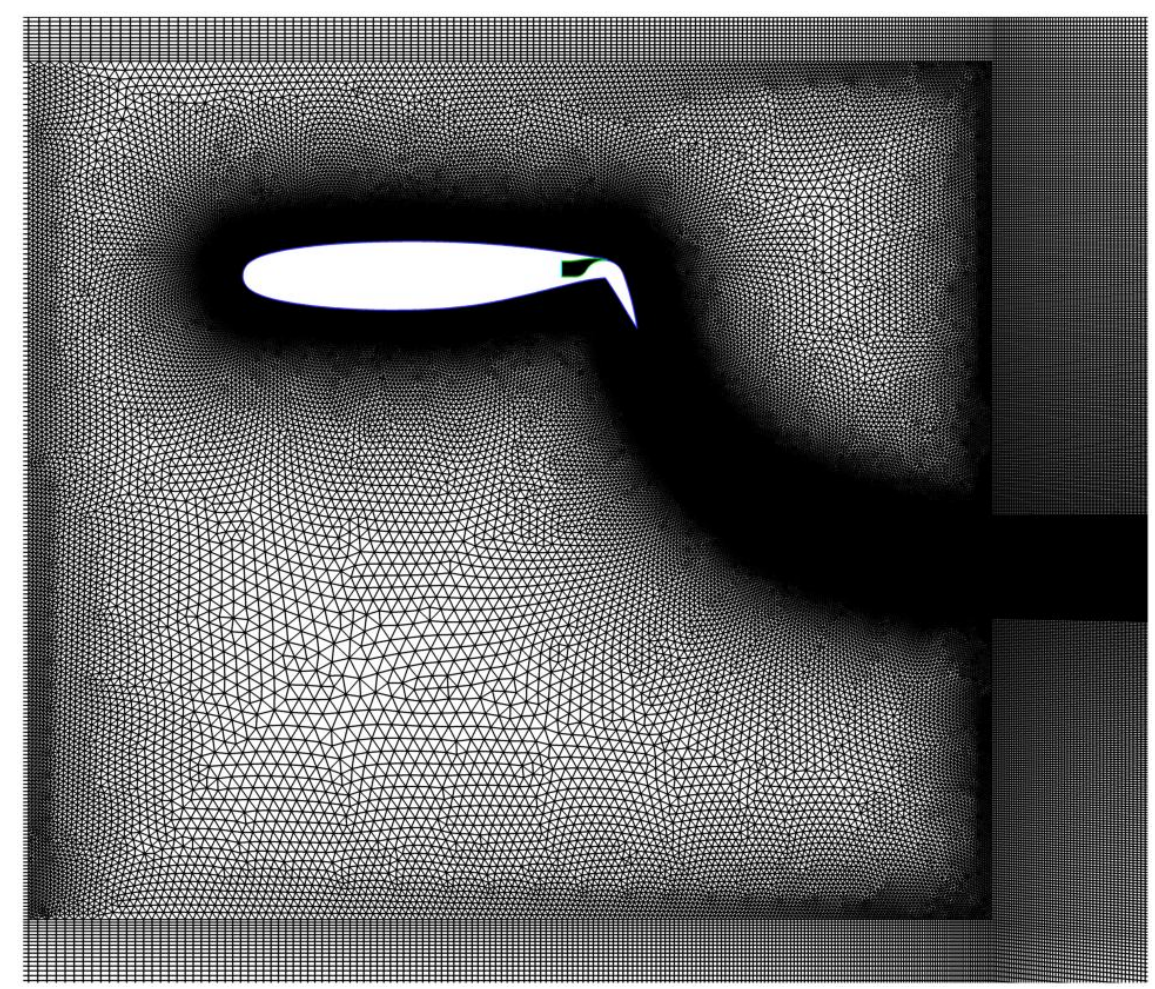

Figure 5-3: Interior unstructured region

Using the CAD program, Pro/ENGINEER Wildfire 4.0, the boundary layer, slot, and wake regions were created and broken into segments. Figure 5-4 shows how these different regions and segments were created. This segmenting scheme allowed structured cell-orientation which promoted orthogonality in the cell-growth for these regions containing highly curved surfaces. Figure 5-5 displays how the cells within this region are kept in an orthogonal orientation with respect to the airfoil surfaces, along with the 
growth of the unstructured interior cells. Keeping this structured, orthogonal cellorientation close to the surfaces of the airfoil and flap keeps the cells from becoming geometrically skewed, along with orienting them in the direction of the flow encountered by these locations. By avoiding cells from being skewed and keeping a high resolution of cells close to the surfaces decreases the computational error in the simulating both the boundary layer region as well as the flow features taking place close to the airfoil. These structured segments of the boundary layer, slot, and wake flow regions start on the airfoil or in the plenum and travel all the way through the domain out the exit of the exterior, to capture the high property gradients of these mixing regions.

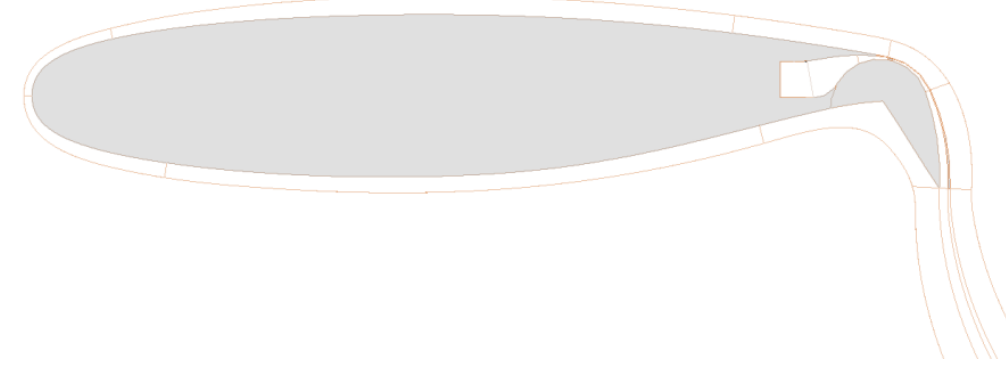

Figure 5-4: Different segments of structured grid

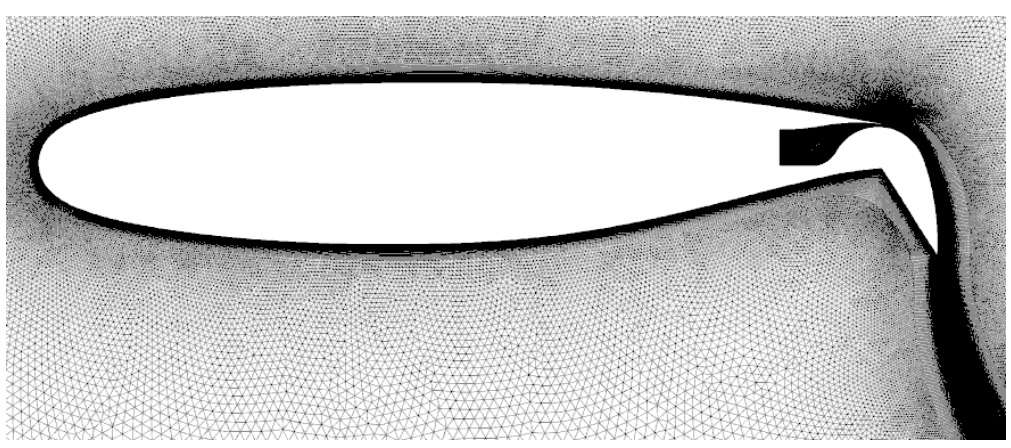

Figure 5-5: Computational grid of airfoil

The first cell height of the boundary layer was specified to target a $y^{+}$value of 1 or lower. This would ensure computational cells within the viscous sublayer to improve accuracy of the solution for the selected turbulence model and prevent the need of wall 
functions. Figure 5-6 presents the details of the slot region with the various different structured segments. Figure 5-7 shows the details of the cell growth as the structured boundary layer region transitions into the unstructured interior.

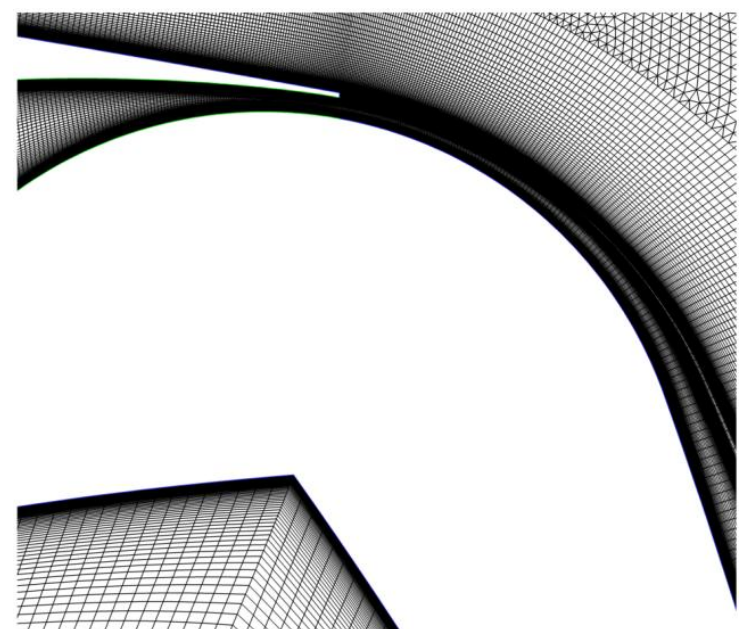

Figure 5-6: Details of the slot region

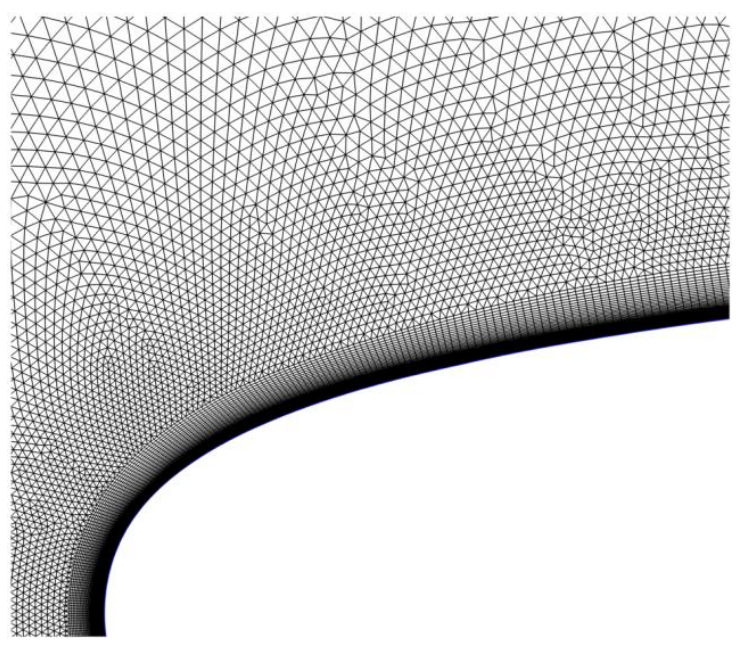

Figure 5-7: Boundary layer transition of mesh

To establish the locations of the high resolution, structured segments of the wake and mixing regions of the mesh, an iterative process was taken on. On the first iteration, these structured segments were placed in estimated locations where the wake was thought to occur. The mesh was then solved until a stabilized flow field was established. The solution was then overlaid on the mesh (Figure 5-8), which was then modified to have the structured regions capture the entire wake and mixing region as described by velocity and turbulent kinetic energy contours. The mesh was solved and modified once more to ensure the full wake and mixing region was confined by the structured mesh segments. 


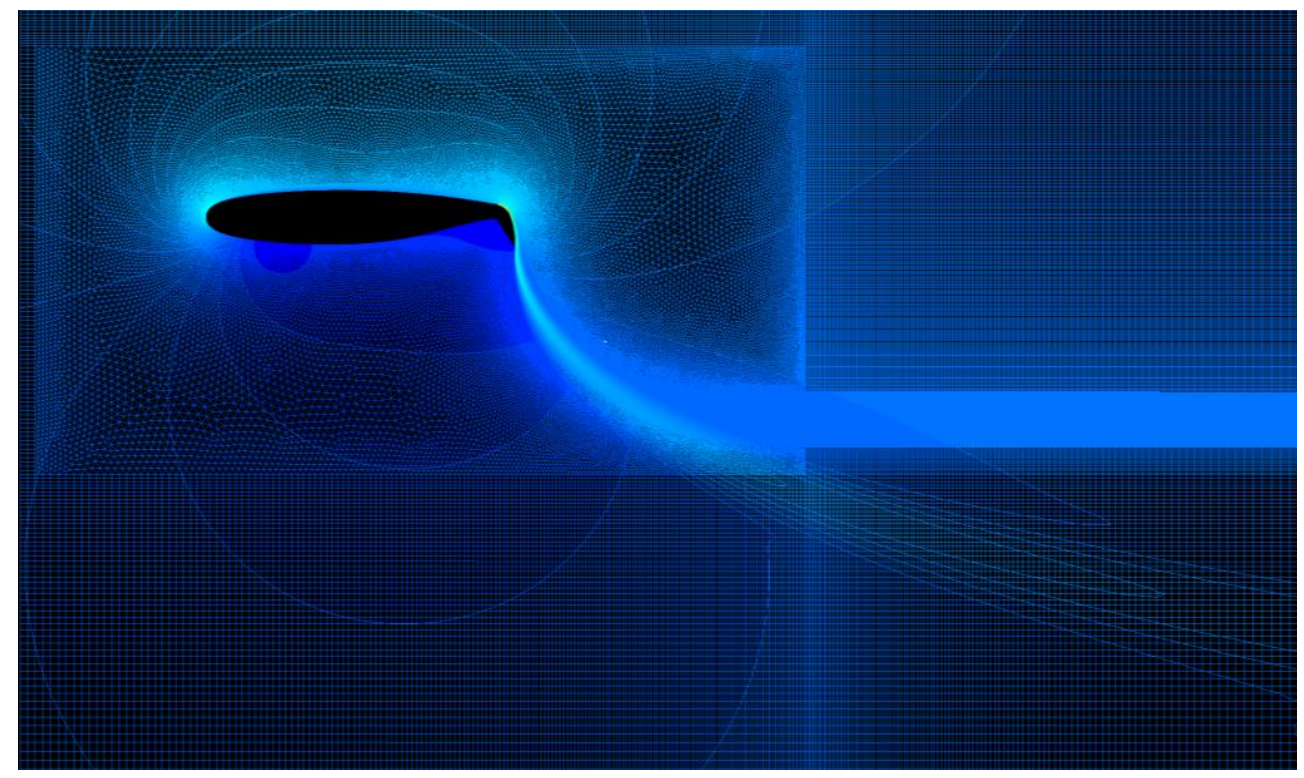

Figure 5-8: Contours of Mach overlaid mesh to shape structured regions

The same meshing techniques were practiced on the $90^{\circ}$ flap configurations as well, including the iterative structured mesh development. Figure 5-9 and Figure 5-10 below display the unstructured interior region and refined flap/wake region of the $90^{\circ}$ flap configuration respectively.

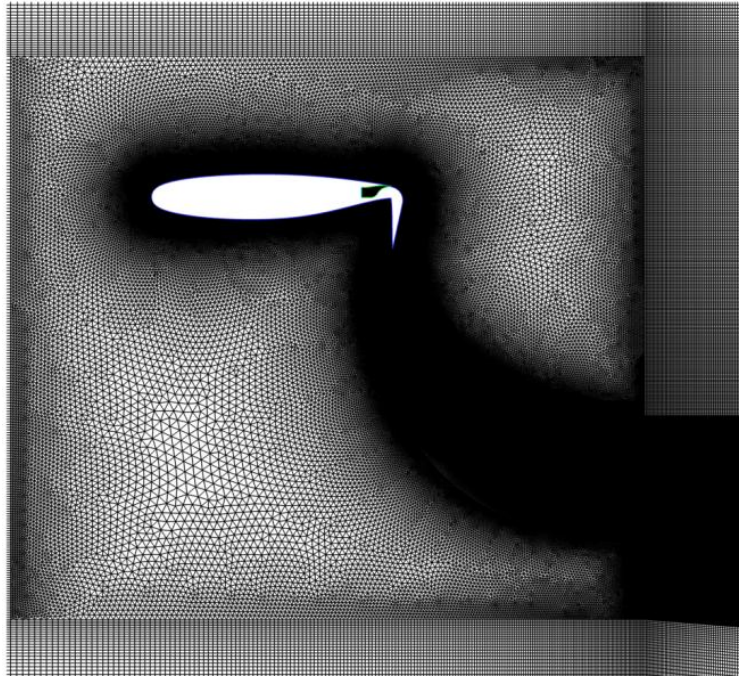

Figure 5-9: Unstructured region of $90^{\circ}$ flap

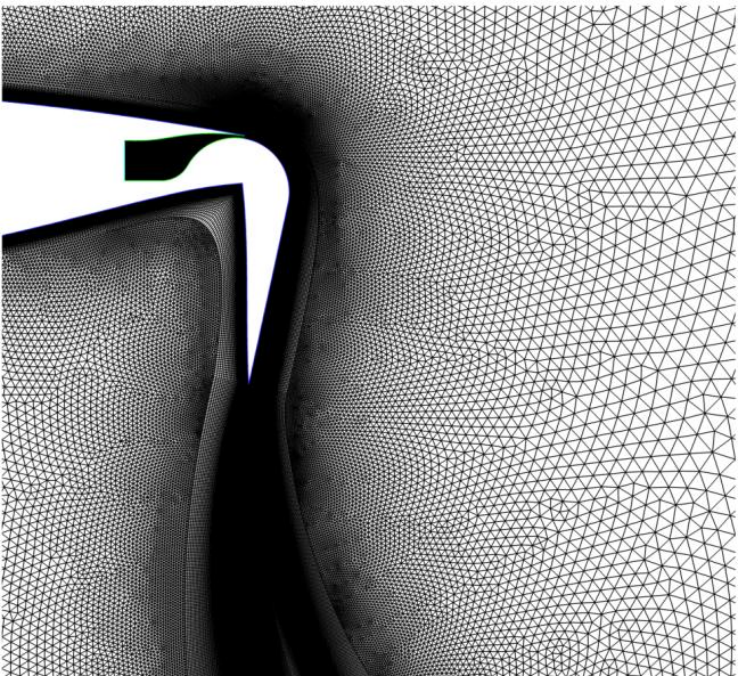

Figure 5-10: Refined structured wake region 
A fully structured C-grid mesh was used for the solving the cruise configuration geometries. A C-grid was chosen to benefit from its ability to maintain cell-orthogonality around the entire airfoil including the round leading edge, along with keeping increased cell-density within the wake of the airfoil to fully capture the mixing occurring behind the airfoil. The first cell height of the boundary layer was again targeted to obtain a $y^{+}$value of 1 , staying consistent with the meshes of the $60^{\circ}$ and $90^{\circ}$ flap deflection configurations. The mesh extends ten chord-lengths above, below, and in front of the airfoil, fifteen chord-lengths aft. Note that the plenum and slot of the airfoil were not modeled in these meshes due to the slots being retracted when in cruise configuration. Figure 5-11 below presents the entire domain of the cruise configuration mesh used, and Figure 5-12 shows the mesh in the region around the airfoil. Figure 5-13 and Figure 5-14 display the details of the cruise configuration mesh near the leading and trailing edge of the airfoil respectively.

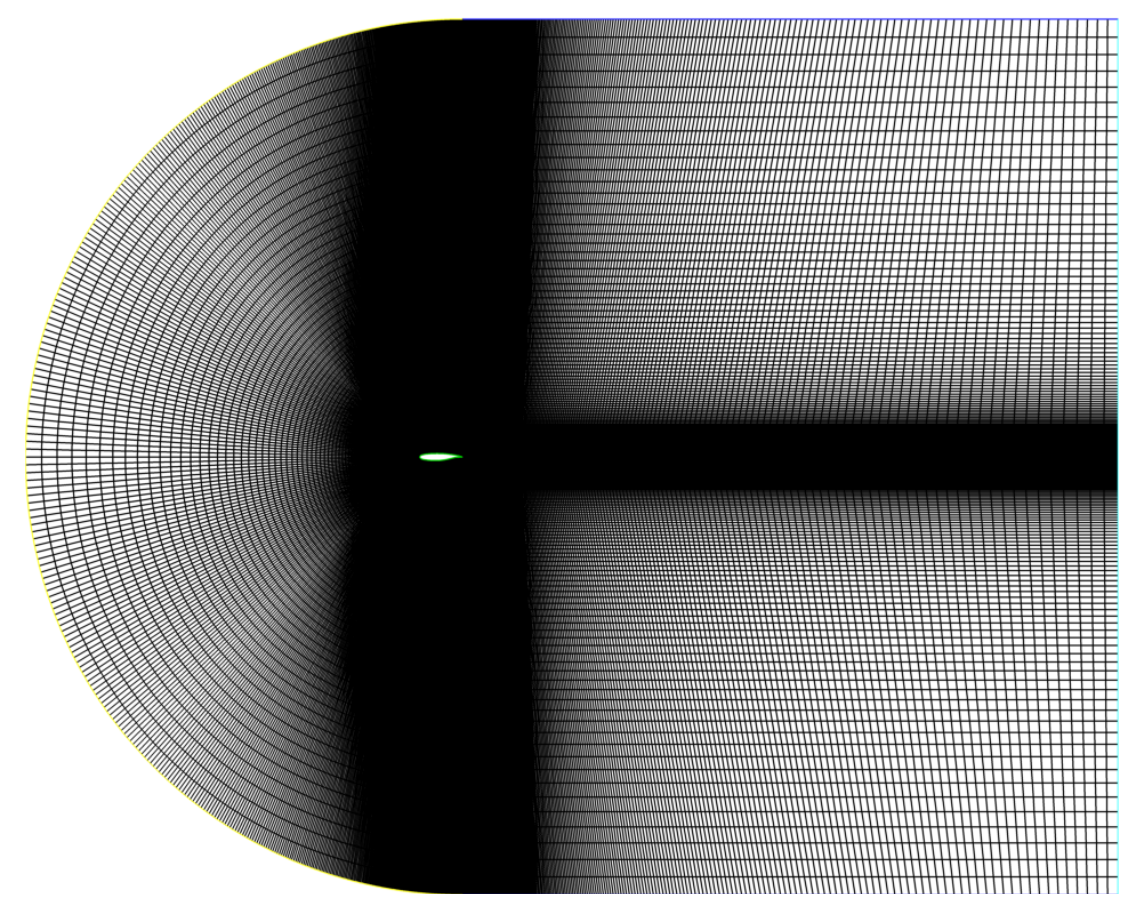

Figure 5-11: C-grid of cruise configuration simulation 


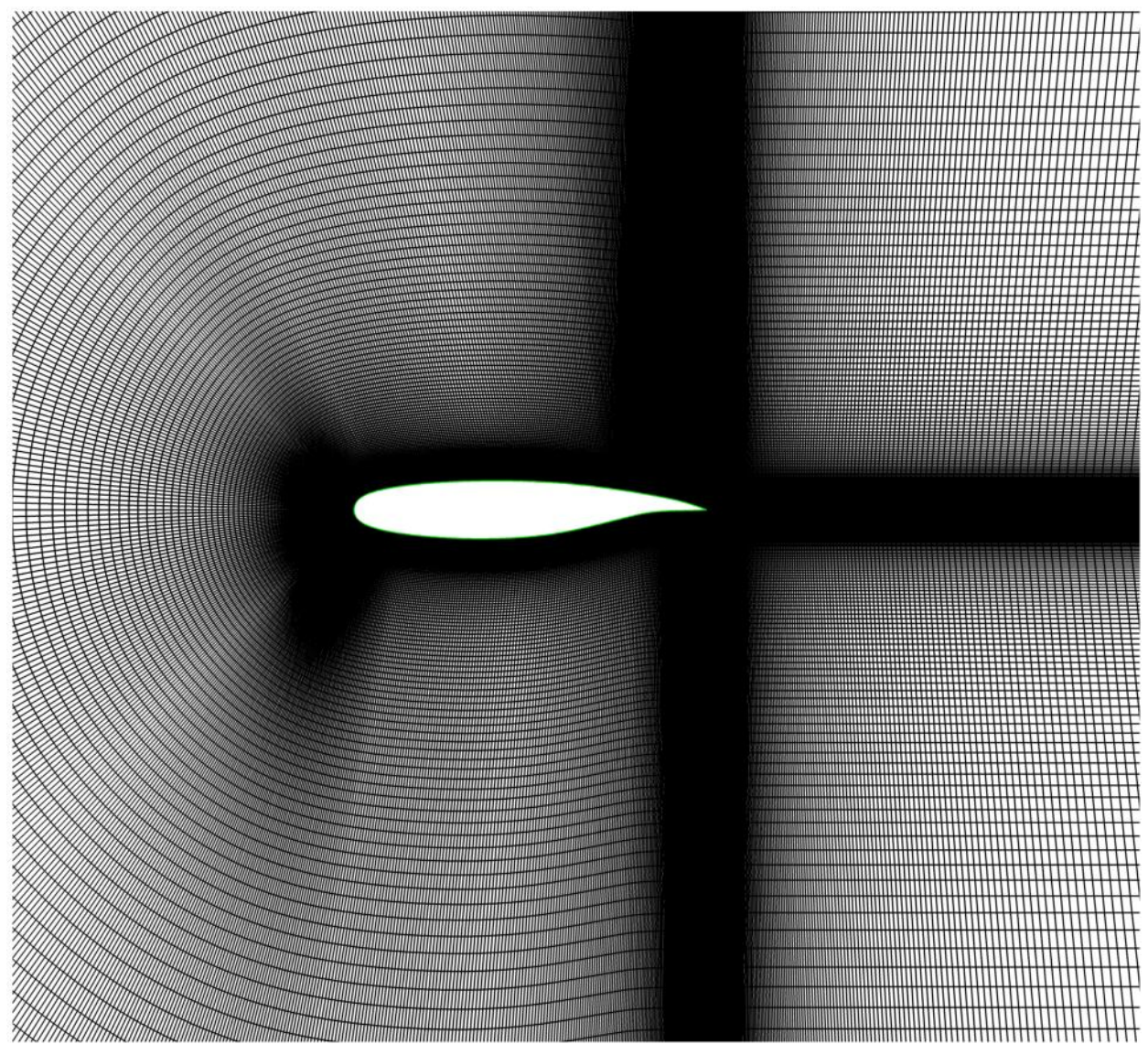

Figure 5-12: Structured mesh around airfoil

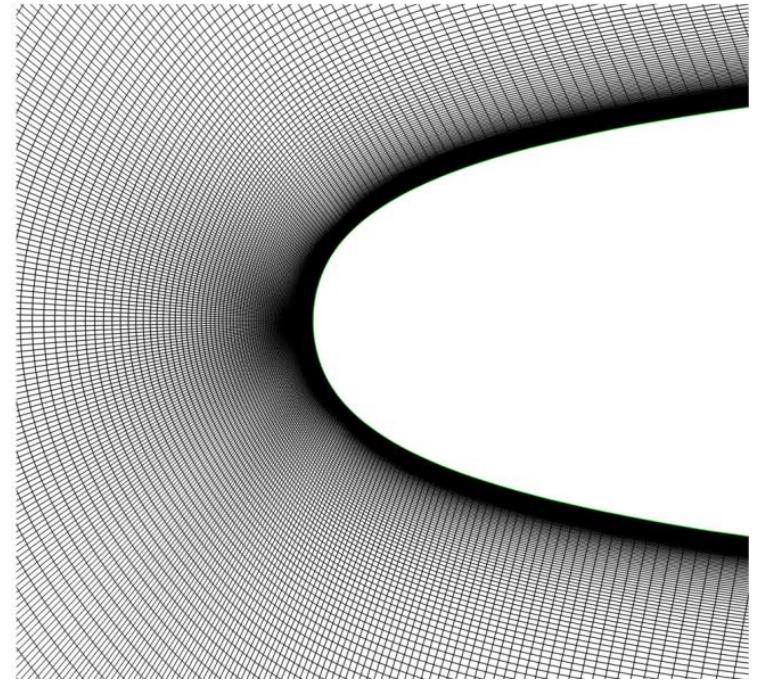

Figure 5-13: Details of leading edge mesh

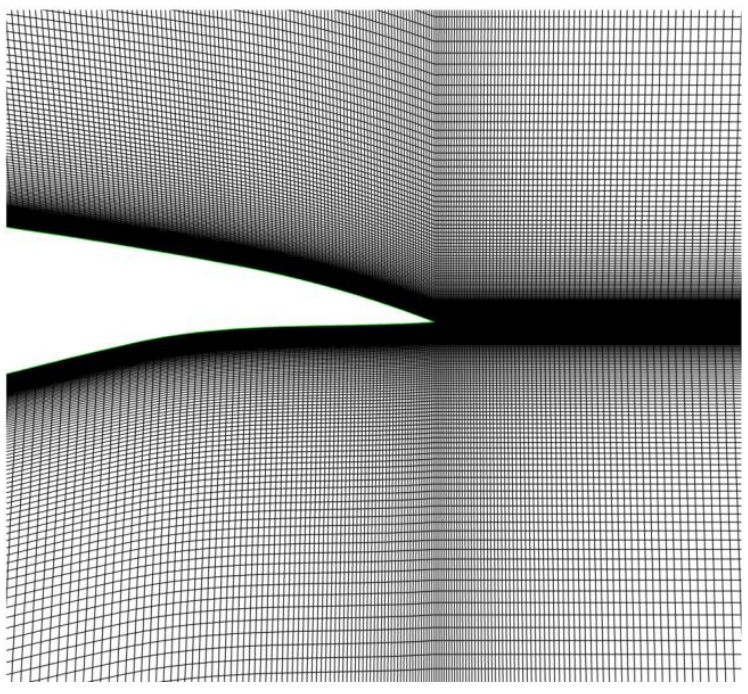

Figure 5-14: Refined wake of trailing edge 


\section{Grid Independence Study}

To ensure the computational mesh resolution applied to the geometry accurately and efficiently captured the flow features of the simulation, a grid independence study was preformed. This was done by developing two different meshes for the same geometry and domain size. The second mesh was generated with approximately half of the cell count that was used on the first mesh, while keeping cell clustering consistent with the first mesh as well. The two meshes (intermediate and coarse) were also solved with the same convergence criteria to keep the solutions consistent. Table 5-2 below presents the specifications of each domain, along with the $\mathrm{C}_{\mathrm{l}}, \mathrm{C}_{\mathrm{d}}$, and $\mathrm{C}_{\mathrm{m} \text {, (c/4) }}$ solutions they produced.

Table 5-2: Mesh details and resulting solutions

\begin{tabular}{|c|c|c|}
\hline Mesh & Coarse & Intermediate \\
\hline Number of Cells $(N)$ & 336,376 & 669,737 \\
\hline Average Cell Size $(\mathrm{h})$ & $1.7242 \mathrm{e}-03$ & $1.2219 \mathrm{e}-03$ \\
\hline $\mathrm{C}_{1}$ & 6.1087 & 6.1033 \\
\hline $\mathrm{C}_{\mathrm{d}}$ & 0.20654 & 0.21071 \\
\hline $\mathrm{C}_{\mathrm{m},(\mathrm{c} / 4)}$ & -1.5133 & -1.5089 \\
\hline
\end{tabular}

Once solutions were obtained for both meshes, they were used to obtain extrapolated solutions for a mesh with an infinite number of cells along with the errors of each of the meshes through the Richardson Extrapolation method. ${ }^{98}$ In this grid independence study, the order of the Fluent solutions ( $n$ ) was approximated to be two. This allowed for the investigation of two different mesh densities to evaluate the extrapolated values of the solutions and the associated errors, rather than the use of three different meshes. 
The first step in evaluating the extrapolated solutions and errors, was determining the average cell size by

$$
h=\left[\frac{1}{N} \sum_{i=1}^{N}\left(\Delta A_{i}\right)\right]^{1 / 2}
$$

where $\Delta \mathrm{A}_{i}$ is the area of the $i^{\text {th }}$ cell, and $N$ is the total number of cells used for each of the meshes. The grid refinement factor is defined as the measure of refinement from one mesh to the next, and can be calculated as

$$
r_{i j}=\frac{h_{i}}{h_{j}}
$$

where $h_{i}$ is the average cell size of the coarse mesh and $h_{j}$ is the average cell size of the intermediate mesh. Note that it is desirable to have a refinement factor above $1.3 .^{98}$ Once these values are determined, the extrapolated value of a solution of an infinite cell mesh can be computed as

$$
\phi_{\infty}=\frac{r_{i j}^{n} \phi_{j}-\phi_{i}}{r_{i j}^{n}-1}
$$

where $\phi_{j}$ is the solution of the intermediate mesh and $\phi_{i}$ is the solution of the coarse mesh. The approximate relative error can be evaluated as

$$
e_{a}^{i j}=\left|\frac{\phi_{j}-\phi_{i}}{\phi_{j}}\right|
$$

And the extrapolated relative error can be calculated as 


$$
e_{\infty}=\left|\frac{\phi_{\infty}-\phi_{j}}{\phi_{\infty}}\right|
$$

The grid convergence index (GCI) can be found using the following

$$
G C I=\frac{1.25 e_{a}^{i j}}{r_{i j}^{n}-1}
$$

The absolute error associated with each of the meshes solutions is

$$
\varepsilon_{i}^{\prime}=\phi_{\infty}-\phi_{i}
$$

The performance criteria of $\mathrm{C}_{\mathrm{l}}, \mathrm{C}_{\mathrm{d}}$, and $\mathrm{C}_{\mathrm{m}}$, (c/4) were all extrapolated and errors analyzed. The Richardson Extrapolation terms and solutions for each of the solutions are presented below in Table 5-3. Figure 5-15 through Figure 5-17 show how each of the solutions converges towards the extrapolated value as the cell count is increased. The associated errors of each of the meshes solutions are also displayed on these figures with error bars.

Table 5-3: Extrapolation terms, solutions, and errors

\begin{tabular}{|c|c|c|c|}
\hline & $\mathbf{C}_{\mathbf{l}}$ & $\mathbf{C}_{\mathbf{d}}$ & $\mathbf{C}_{\mathbf{m},(\mathbf{c} / \mathbf{4})}$ \\
\hline $\mathbf{r}_{\mathbf{1 2}}$ & \multicolumn{3}{|c|}{1.4110} \\
\hline $\boldsymbol{\phi}_{\infty}$ & 6.0979 & 0.21492 & -1.5045 \\
\hline $\boldsymbol{e}_{a}^{12}$ & $0.08848 \%$ & $1.9790 \%$ & $0.29160 \%$ \\
\hline $\boldsymbol{e}_{\infty}$ & $0.08936 \%$ & $1.9578 \%$ & $0.29511 \%$ \\
\hline $\boldsymbol{G} \boldsymbol{C I}$ & 0.00112 & 0.02496 & 0.00368 \\
\hline $\boldsymbol{\varepsilon}_{\boldsymbol{1}}$ & -0.0108 & 0.00838 & 0.0088 \\
\hline $\boldsymbol{\varepsilon}_{\mathbf{2}}$ & -0.0054 & 0.00421 & 0.0044 \\
\hline
\end{tabular}




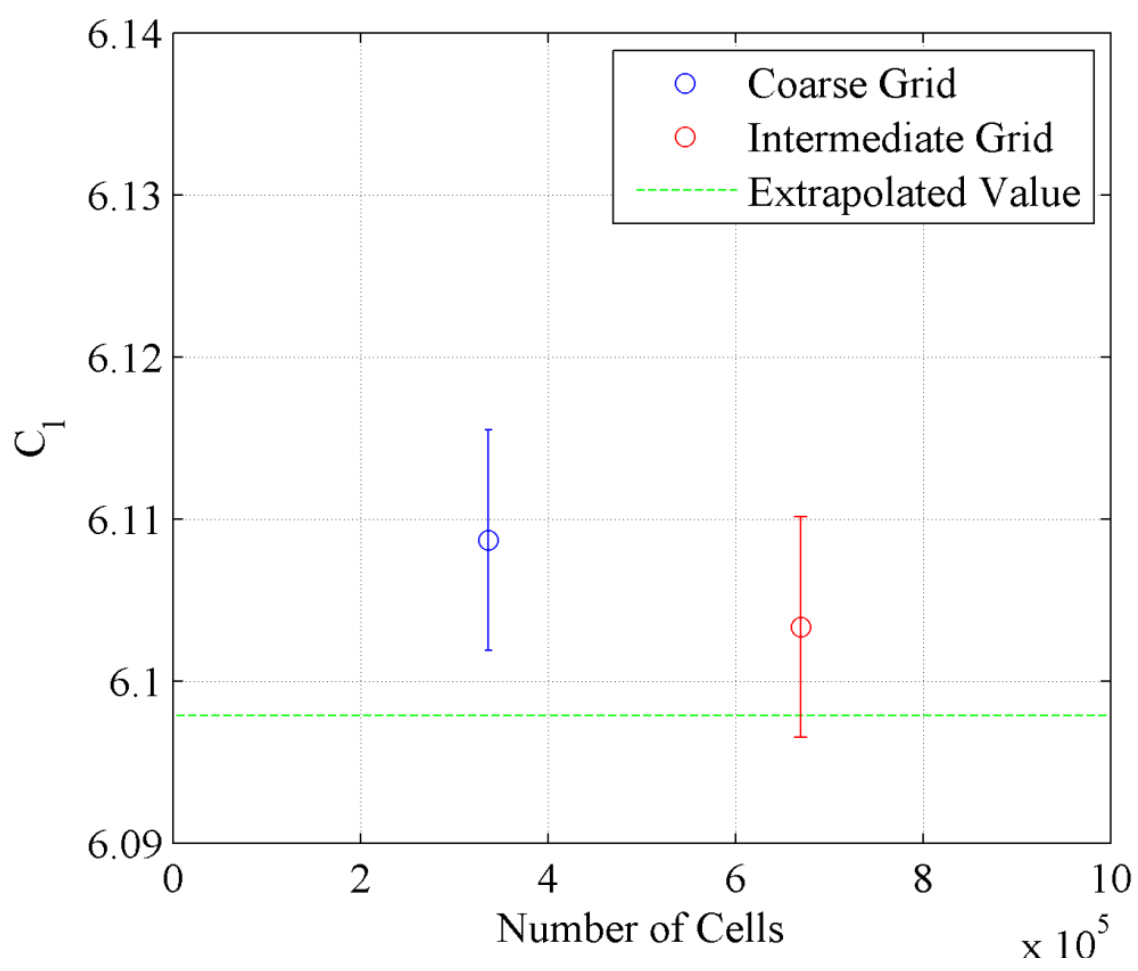

Figure 5-15: Solved and extrapolated lift coefficient results

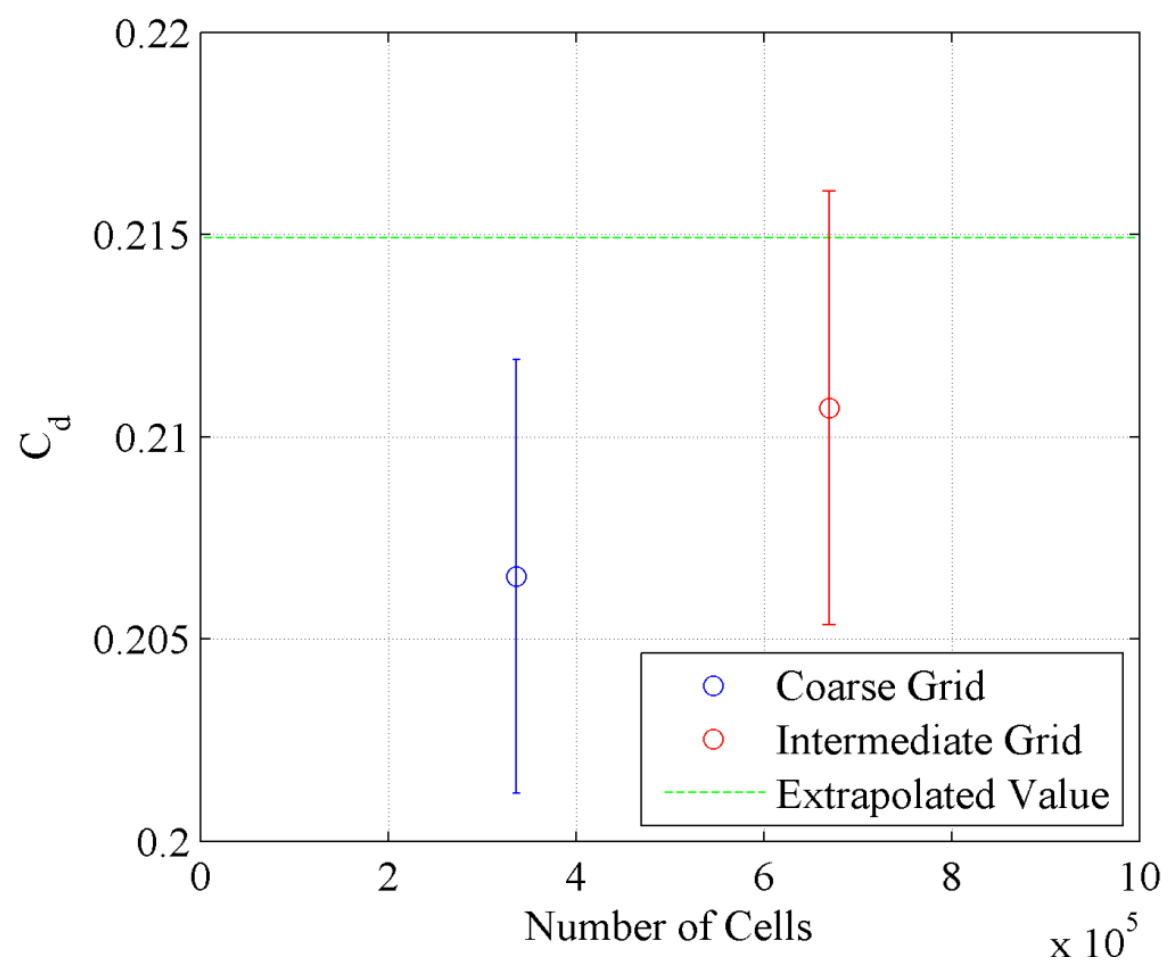

Figure 5-16: Solved and extrapolated drag coefficient results 


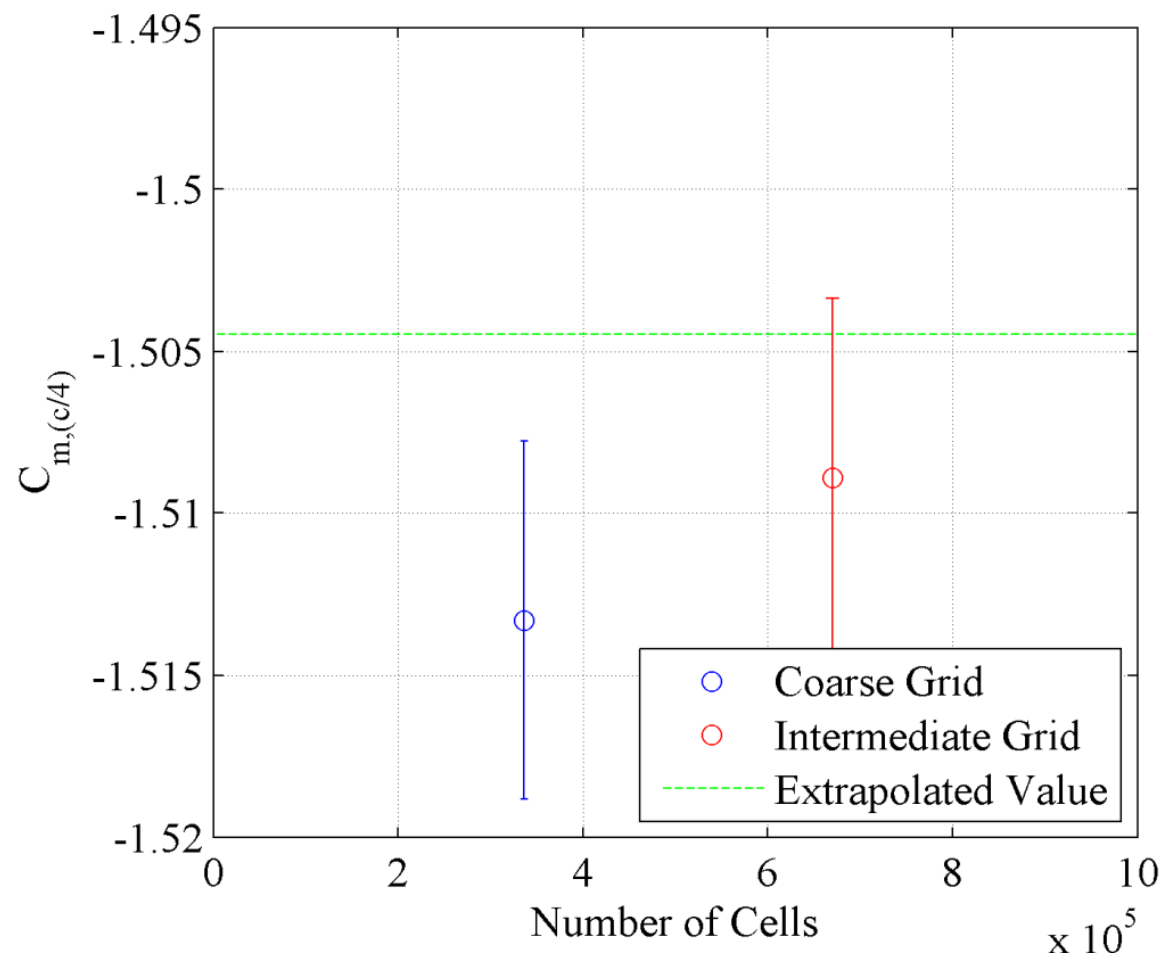

Figure 5-17: Solved and extrapolated moment coefficient results

From Figure 5-15 above, the lower-density mesh over-predicts $C_{1}$. This relationship can be attributed to not having a high enough resolution of cells in areas of high property gradients. For example, the region located directly between the slot flow and the flow over the top of the airfoil experiences a large amount of mixing, causing large pressure and turbulent kinetic energy gradients. By not resolving this area enough, it does not capture the proper flow physics of the mixing flows and greatly damps out the products of these gradients. This would not capture the entirety of the turbulence and vorticities introduced, resulting in a cleaner and more continuous flow field around the airfoil enhancing $C_{1}$.

Observing the behaviors of both $\mathrm{C}_{\mathrm{d}}$ and $\mathrm{C}_{\mathrm{m},(\mathrm{c} / 4)}$ as cell count is increased, shown in Figure 5-16 and Figure 5-17, they both increase in value. The increase in drag can be credited to the same causes that caused the over-prediction in lift. Since the lower 
density of cells over-simplifies the flow in regions of high property flux, it does not properly capture all of the mixing flow features which would be increasing drag. The decreasing moment relation is a product of this occurrence as well. Since the region of the highest property gradients is located near the slot on the trailing edge of the airfoil, this is the place where the flow features are not captured as accurately with the lower cell counts. Thus, increasing the negative pressures encountered on the upper surface in this region. This event both increases the lift and negative moment characteristics of the airfoil.

The intermediate mesh was deemed to be an adequate cell-density for simulation due to its proximity to the extrapolated solutions, with its associated errors encompassing the extrapolated values. In doing so, this established a cell count and density that provided solutions with low discretization error. Although a mesh with a higher cell count could produce lower error, with the relationships observed from this study, in doing so it would produce little improvement with a large associated increase computing resources.

\section{Validation}

To compare the simulation methods used in this investigation to other published data, a validation case was performed on a GTRI-DR CC airfoil which had both numerical and experimental data available. ${ }^{52}$ The GTRI-DR geometry that was used in this case study is displayed below in Figure 5-18. 


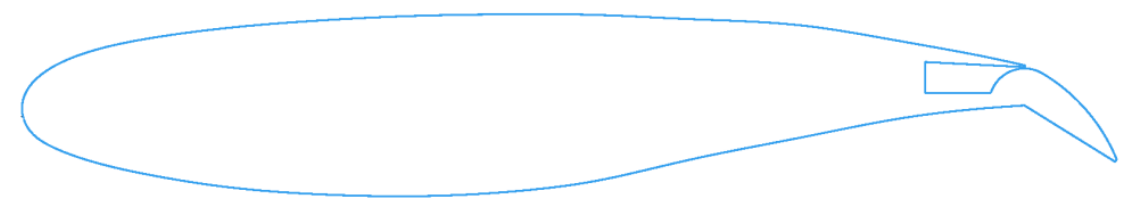

Figure 5-18: GTRI-DR CC airfoil geometry

Throughout this case study, all of the settings of the published results were matched except the gridding techniques and turbulence model. The gridding techniques that were applied to the present research were utilized for the validation case to measure the numerical differences that could arise with the different techniques used. The previous case used the Spalart-Allmaras ${ }^{94}$ turbulence model (FUN3D) and a blend between the Spalart-Allmaras and Menter SST ${ }^{99}$ turbulence model (CFL3D) to simulate the numerical data, where the realizable $\mathrm{k}-\varepsilon^{84}$ was used in the present case study to stay consistent with the turbulence model of the presented research. Table 5-4 below describes all of the boundary conditions used to match this previous study.

Table 5-4: Validation boundary conditions and settings

\begin{tabular}{|c|c|c|c|}
\hline \multicolumn{2}{|c|}{ Freestream Conditions } & \multicolumn{2}{c|}{ Geometry } \\
\hline $\mathrm{M}_{\infty}$ & 0.0842 & $\mathrm{c}(\mathrm{m})$ & 0.2032 \\
\hline$\rho_{\infty}\left(\mathrm{kg} / \mathrm{m}^{3}\right)$ & 1.225 & $\mathrm{c}^{\prime}(\mathrm{m})$ & 0.1994 \\
\hline $\mathrm{T}_{\infty}(\mathrm{K})$ & 288.15 & $\delta_{\mathrm{f}}\left({ }^{\circ}\right)$ & 30 \\
\hline $\mathrm{P}_{\infty}(\mathrm{Pa})$ & 101,325 & $\mathrm{c}_{\mathrm{f}} / \mathrm{C}$ & 0.0955 \\
\hline$\mu_{\infty}\left(\mathrm{kg} /\left(\mathrm{m}^{*} \mathrm{sec}\right)\right)$ & $1.81205^{*} 10^{-5}$ & $\mathrm{~h}_{\mathrm{slo}} / \mathrm{C}$ & 0.00191 \\
\hline $\mathrm{R}_{\infty}\left(\mathrm{J} /\left(\mathrm{kg}^{*} \mathrm{~K}\right)\right)$ & 287.058 & & \\
\hline $\mathrm{q}_{\infty}\left(\mathrm{kg} /\left(\mathrm{m}^{*} \mathrm{sec}^{2}\right)\right)$ & 502.86 & & \\
\hline
\end{tabular}

To compare the previous numerical data, experimental data, and resulting case study numerical data, $\mathrm{C}_{\mu}$ values were matched along a $\mathrm{C}_{1}$ vs. $\mathrm{C}_{\mu}$ curve provided by the published research. Several $C_{\mu}$ values were chosen within the proximity of published data points and the chosen value for this investigation, which corresponded to values 
ranging from 0.2 to 0.27 . Figure 5-19 below presents the lift augmentation results found during this case study, with the produced results labeled "Solution".

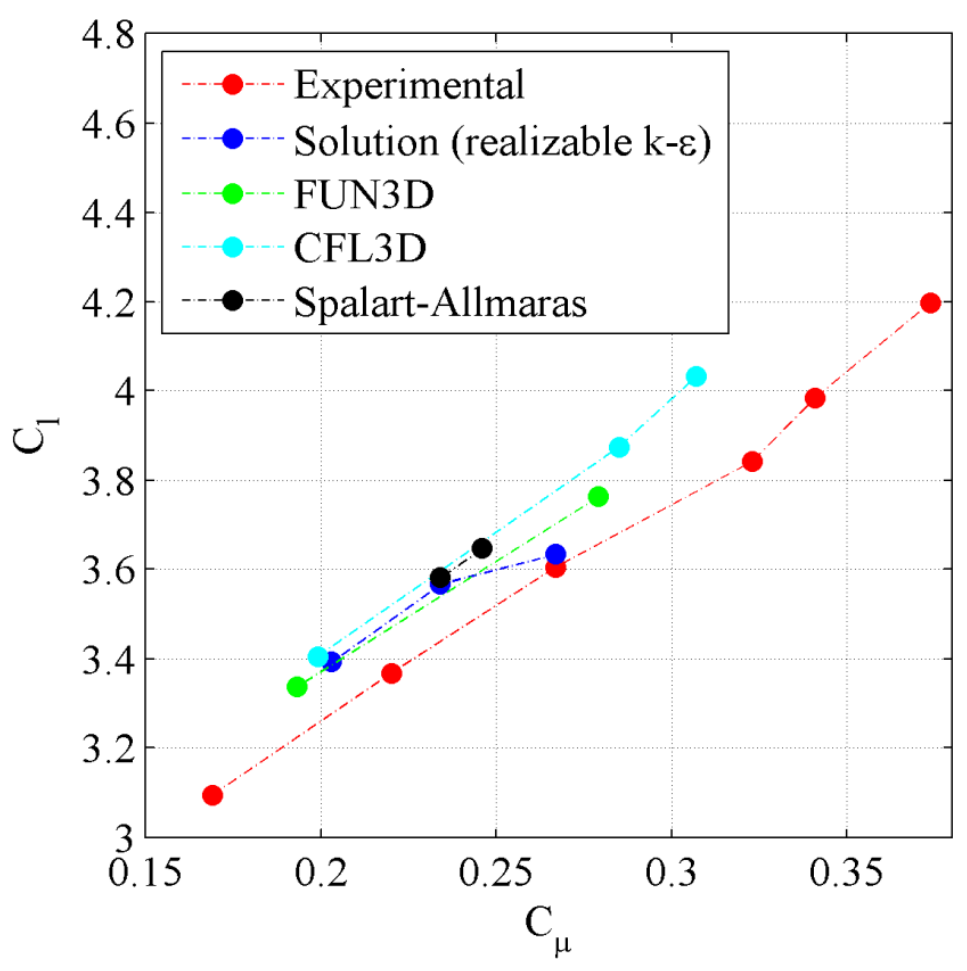

Figure 5-19: Lift coefficient given momentum coefficient comparison

The above plot shows that the results produced by the current research methodology stayed within $1 \%$ of the published numerical results at the $\mathrm{C}_{\mu}$ values below 0.25 , which was approximately $3.5 \%$ above the experimental results. As $\mathrm{C}_{\mu}$ was increased, the realizable k- $\varepsilon$ used in this research approached experimental results with only a $1 \%$ over-prediction of $\mathrm{C}_{1}$. This showed improvement over the published results, which were consistently $3.5 \%$ over the experimental $C_{1}$ values. The over-prediction of lift by the numerical results above is due to their over-prediction of turbulent kinetic energy ${ }^{56}$ and failure to follow the streamline turning of the CC flow field. ${ }^{7}$ Next, the $C_{p}$ distribution of the solution at $\mathrm{C}_{\mu}$ of 0.27 was plotted against the published data to further examine the differences in Figure 5-20. 


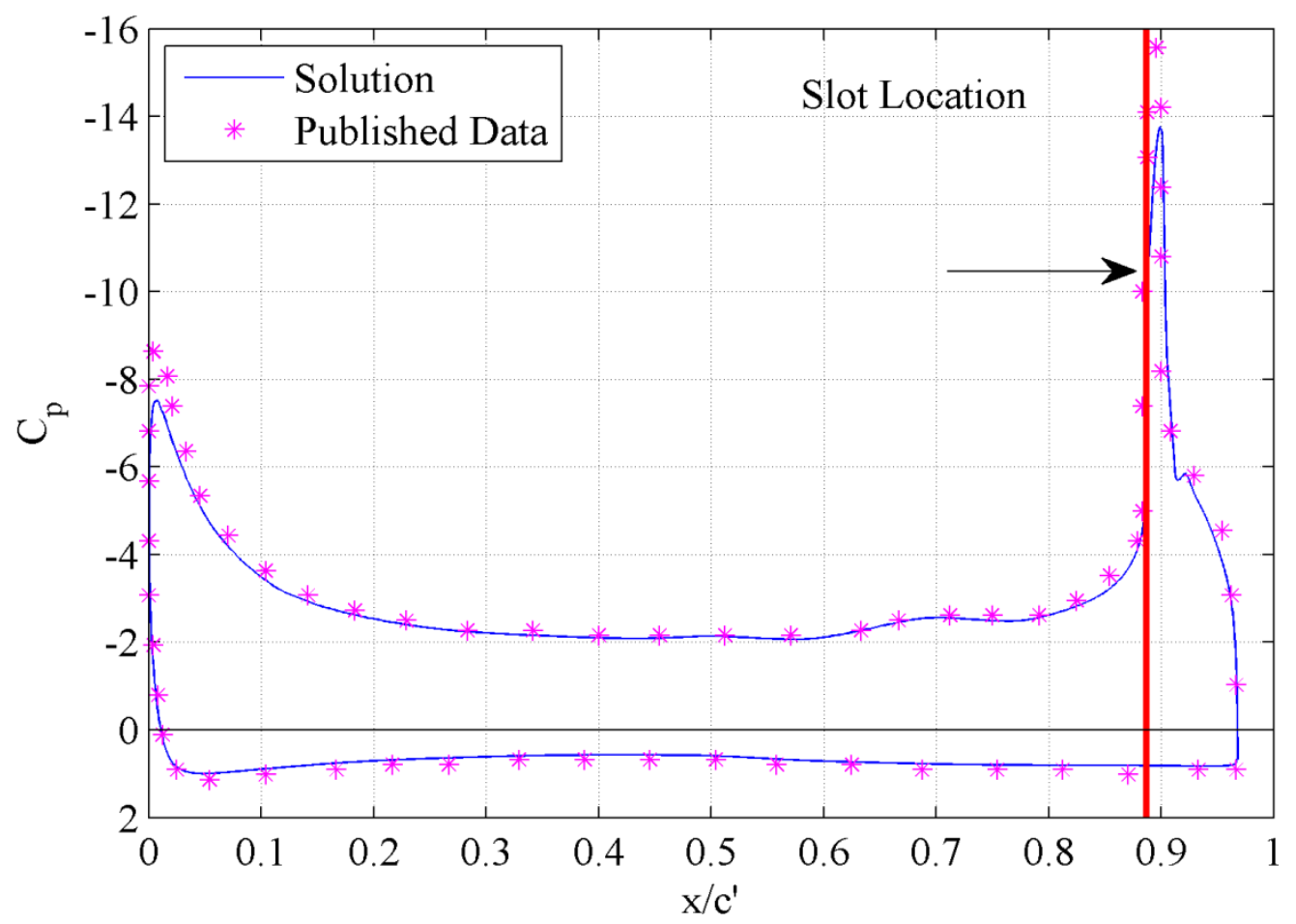

Figure 5-20: Airfoil pressure coefficient distribution comparison

The overall match of the $\mathrm{C}_{\mathrm{p}}$ data is excellent, with a slight under prediction of the current research method at the locations of the leading edge and trailing edge suction peaks. This under prediction of the suction peaks is reflected in the previous lift values presented in Figure 5-19, where the current methods under predict $\mathrm{C}_{1}$. Again this shows that the numerical methods in this research produced results that reasonably agree with corresponding published numerical data, and even experimental data in some cases.

Figure 5-21, below, displays the $\mathrm{y}^{+}$values of the current research methods validation case to examine the quality of the grid resolution. 


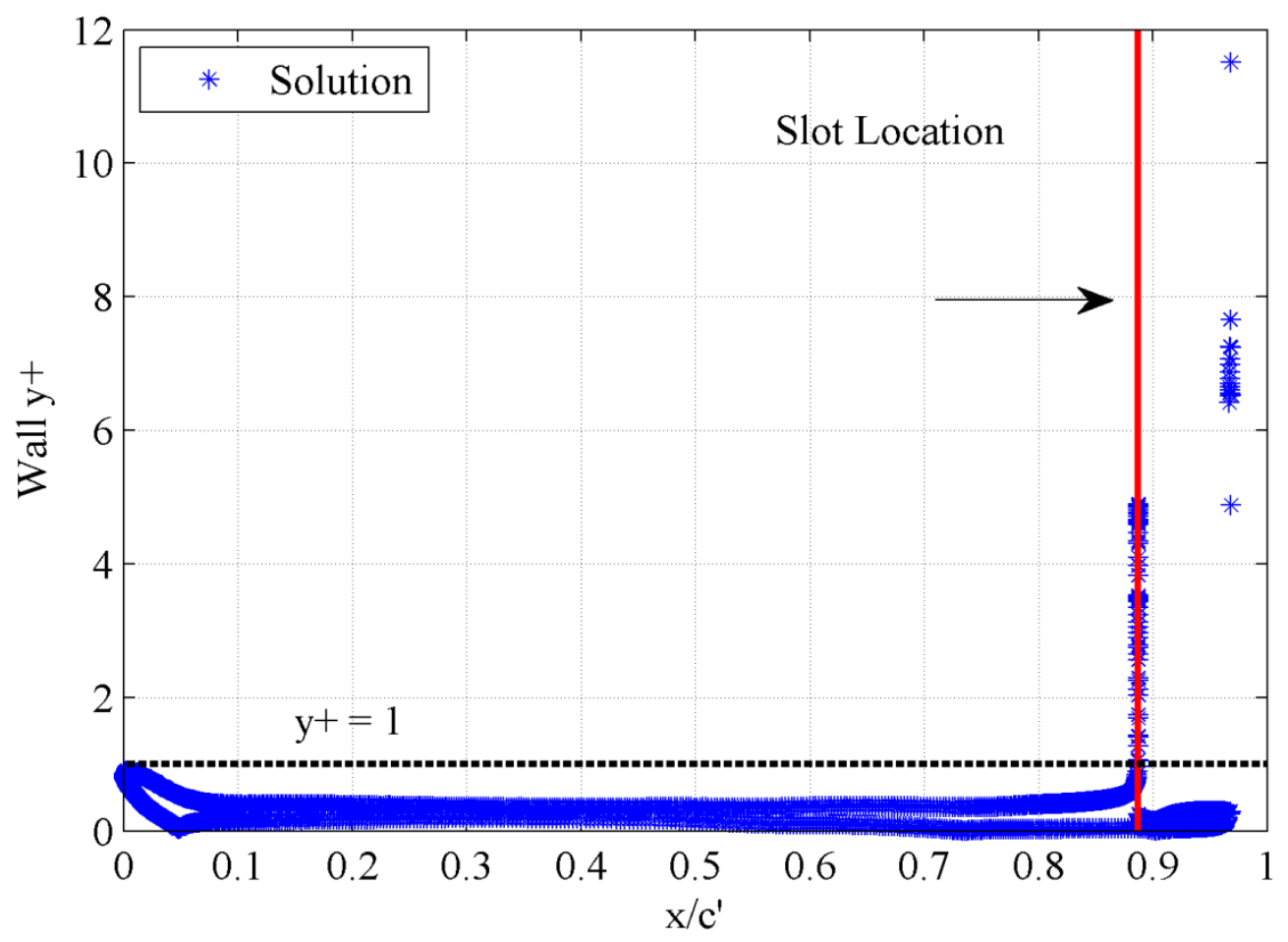

Figure 5-21: Resulting $y^{+}$values of validation case

The $\mathrm{y}^{+}$values encountered stayed below one, with the exception of the slot location and the blunt trailing edge of the flap. The increased $\mathrm{y}^{+}$values were also experienced in the published research, ${ }^{52}$ with further investigation into increasing grid density at the slot location showing no improvements. The $\mathrm{y}^{+}$values at the trailing edge are believed to increase dramatically due to flow separation at the very large, blunt trailing edge of the GTRI-DR geometry provided. Overall, the grid was deemed adequate for the chosen turbulence model, thus confirming the results.

With the current gridding methods and turbulence model producing results behaving consistent with the published numerical results, and even with experimental data at higher $\mathrm{C}_{\mu}$ values, they were deemed acceptable for the analysis at hand. 


\section{Boundary Conditions}

Two different flight conditions were analyzed with the flap settings for each of the six different $\mathrm{CC}$ flap configurations to map their performance and aerodynamic characteristics at these various conditions. The first flight condition was at sea level altitude and low airspeed to examine the takeoff and approach/landing performance with the slot open and blowing set to a $\mathrm{C}_{\mu}$ of 0.3 . This flight condition was applied to the takeoff and approach/landing flap settings, which corresponded to deflections of $60^{\circ}$ and $90^{\circ}$ respectively. The second flight condition was at a transonic cruise velocity of 0.74 Mach and altitude of $30,000 \mathrm{ft}$, which was taken from the cruise design point of the AMELIA aircraft design. This flight condition was only applied to the cruise flap setting, or $0^{\circ}$ of flap deflection with no blowing slot. The cruise $\alpha$ of each flap configuration was adjusted until the cruise target 3-D $\mathrm{C}_{\mathrm{L}}$ of 0.247 (determined by AMELIA design) was achieved.

To achieve the desired blowing conditions of the low airspeed flight condition, the following calculations were made. As previously mentioned, a moderate momentum coefficient $\left(\mathrm{C}_{\mu}\right)$ of 0.3 was targeted for the takeoff and approach/landing analysis. The target 2-D $\mathrm{C}_{\mu}$ was calculated using

$$
C_{\mu}=\frac{\dot{m}_{s l o t} * V_{s l o t}}{q_{\infty} * c}
$$

where $\dot{m}_{\text {slot }}$ is the 2-D slot mass flow rate calculated using only the slot height. The goal of the slot flow was to stay below sonic, keeping the complexity of the plenum and slot design down and not having to develop and manufacture an intricate converging- 
diverging nozzle for the slots of the AMELIA wind tunnel model. Having sonic slot flow would also be impractical in achieving the heightened noise reduction goals set forth by NASA's $\mathrm{N}+2$ definition, which the AMELIA wind tunnel model was designed to. Though it should be noted that above sonic flow has been seen to not have negative effects on the Coanda flow of CC, but yield additional lift gains. ${ }^{2,4,12,100}$ To ensure sonic flow was not achieved, the critical area of the slot was computed using

$$
\frac{A}{A^{*}}=\frac{1}{M_{\text {slot }}} *\left[\left(\frac{2}{\gamma+1}\right) *\left(1+\frac{\gamma-1}{2} * M_{\text {slot }}{ }^{2}\right)^{\frac{\gamma+1}{2 *(\gamma-1)}}\right]
$$

With this equation, the design geometry, and sea level static conditions, a plot of $\mathrm{C}_{\mu}$ vs. $\mathrm{M}_{\infty}$ was constructed with a red sonic slot area to visualize the acceptable design region of the slot. This graph is presented in Figure 5-22 below, with the chosen design point which represents a freestream Mach of 0.1 or 66 knots as seen by the airfoil section. It should be noted that due to the AMELIA design with having a quarter-chord wing sweep $\left(\Lambda_{(\mathrm{c} / 4)}\right)$ of $34^{\circ}$, the actual freestream seen by the aircraft is approximately 0.12 Mach or 80 knots. Throughout this investigation the effective velocity observed by the airfoil section will be referred to as the freestream Mach or $\mathrm{M}_{\infty}$. 


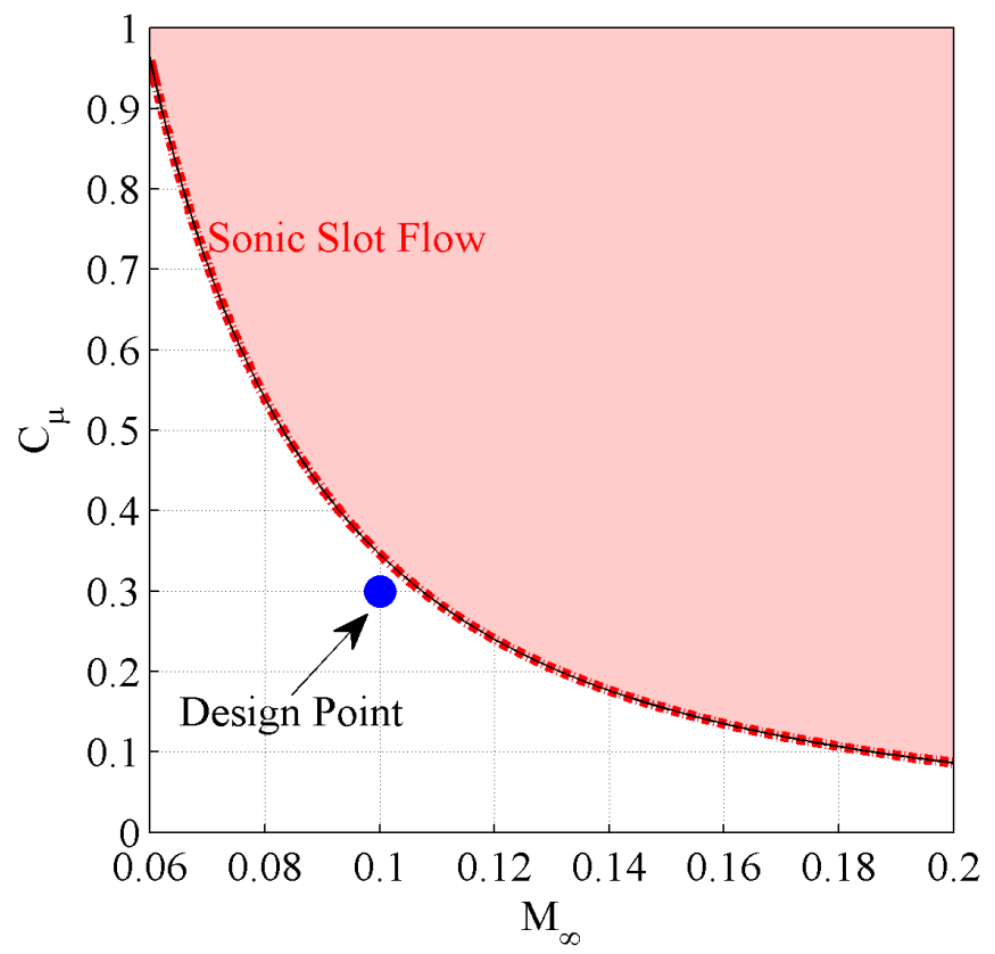

Figure 5-22: Maximum subsonic $C_{\mu}$ attainable

From the chosen $\mathrm{M}_{\infty}$, sea level static conditions, and equation (35), the slot boundary conditions were computed. To do this, the 2-D mass low rate had to be derived from the mass flow definition and equation (35). The resulting equation follows

$$
\dot{m}_{s l o t}=\sqrt{\frac{C_{\mu} * P_{s l o t} * h_{s l o t} * q_{\infty} * c}{R_{\infty} * T_{\infty}}}
$$

where $\mathrm{P}_{\text {slot }}$ represents the static pressure at the exit of the slot, which has been measured from previous analysis of the geometry, and $\dot{m}_{\text {slot }}$ represents the 2-D mass flow rate per span. From equation (37) and equation (35), $\mathrm{V}_{\text {slot }}$ and $\mathrm{M}_{\text {slot }}$ were backed out. This was then used to find the stagnation pressure and temperature at the slot assuming freestream static temperature at the slot exit and with the following equations 


$$
\begin{gathered}
P_{\text {stag }}=P_{\text {slot }} *\left(1+\frac{\gamma-1}{2} * M_{\text {slot }}^{2}\right)^{\frac{\gamma}{(\gamma-1)}} \\
T_{\text {stag }}=T_{\text {slot }} *\left(1+\frac{\gamma-1}{2} * M_{\text {slot }}^{2}\right)
\end{gathered}
$$

During the cruise condition cases, the 3-D lift curve for each flap was approximated from the 2-D lift curve using the following equation ${ }^{101}$

$$
C_{L \alpha}=\frac{A R * C_{l \alpha}}{\frac{C_{l \alpha}}{\pi}+\sqrt{A R^{2}\left(1+\frac{\tan ^{2} \Lambda_{(c / 2)}}{\beta^{12}}\right)+\left(\frac{C_{l \alpha}}{\pi}\right)^{2}}}
$$

where $\mathrm{C}_{\mathrm{L} \alpha}$ is the 3-D lift curve, AR is AMELA's aspect ratio(5.664), $\Lambda_{(\mathrm{c} / 2)}$ is the halfchord aircraft wing sweep angle $\left(28.1^{\circ}\right), \mathrm{C}_{\mathrm{l} \alpha}$ is the 2-D sectional lift curve, and $\beta^{\prime}$ is defined as

$$
\beta^{\prime}=\sqrt{1-M_{\infty}^{2}}
$$

with $\mathrm{M}_{\infty}$ being the freestream Mach (0.74).

Table 5-5 below presents all of the boundary conditions that were used in Fluent for the simulation of both flight conditions. 
Table 5-5: Boundary conditions for all simulations

\begin{tabular}{|c|c|c|c|c|}
\hline & \multicolumn{2}{|c|}{ Freestream Conditions } & \multicolumn{2}{|c|}{ Slot/Flap Conditions } \\
\hline \multirow{7}{*}{ 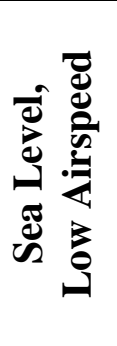 } & $\left(M_{\infty}\right)_{\text {eff }}$ & 0.1 & $\mathrm{M}_{\text {slot }}$ & 0.8128 \\
\hline & $\rho_{\infty}\left(\mathrm{kg} / \mathrm{m}^{3}\right)$ & 1.225 & $\mathrm{~h}_{\text {slot }}(\mathrm{m})$ & $1.1274 * 10^{-3}$ \\
\hline & $\mathrm{T}_{\infty}(\mathrm{K})$ & 288.15 & $\mathrm{P}_{\text {stag }}(\mathrm{Pa})$ & 148,220 \\
\hline & $\mathrm{P}_{\infty}(\mathrm{Pa})$ & 101,325 & $\mathrm{~T}_{\text {stag }}(\mathrm{K})$ & 326.20 \\
\hline & $\mu_{\infty}(\mathrm{kg} /(\mathrm{m} * \mathrm{sec}))$ & $1.8121 * 10^{-5}$ & $\mathrm{C}_{\mu}$ & 0.3 \\
\hline & $\mathrm{R}_{\infty}(\mathrm{J} /(\mathrm{kg} * \mathrm{~K}))$ & 287.06 & $\delta_{\mathrm{f}}\left({ }^{\circ}\right)$ & $60 \& 90$ \\
\hline & $\mathrm{q}_{\infty}\left(\mathrm{kg} /\left(\mathrm{m}^{*} \sec ^{2}\right)\right)$ & 709.29 & & \\
\hline \multirow{7}{*}{ 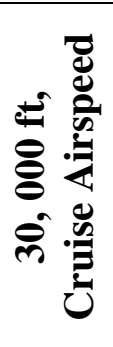 } & $\left(\mathrm{M}_{\infty}\right)_{\mathrm{eff}}$ & 0.6135 & $\mathrm{M}_{\text {slot }}$ & 0 \\
\hline & $\rho_{\infty}\left(\mathrm{kg} / \mathrm{m}^{3}\right)$ & 0.4583 & $\mathrm{~h}_{\text {slot }}(\mathrm{m})$ & 0 \\
\hline & $\mathrm{T}_{\infty}(\mathrm{K})$ & 228.71 & $\mathrm{P}_{\text {stag }}(\mathrm{Pa})$ & N/A \\
\hline & $\mathrm{P}_{\infty}(\mathrm{Pa})$ & 30,089 & $\mathrm{~T}_{\text {stag }}(\mathrm{K})$ & N/A \\
\hline & $\mu_{\infty}\left(\mathrm{kg} /\left(\mathrm{m}^{*} \mathrm{sec}\right)\right)$ & $1.4997 * 10^{-5}$ & $\mathrm{C}_{\mu}$ & 0 \\
\hline & $\mathrm{R}_{\infty}(\mathrm{J} /(\mathrm{kg} * \mathrm{~K}))$ & 287.06 & $\delta_{\mathrm{f}}\left({ }^{\circ}\right)$ & 0 \\
\hline & $\mathrm{q}_{\infty}\left(\mathrm{kg} /\left(\mathrm{m}^{*} \mathrm{sec}^{2}\right)\right)$ & $7,927.63$ & $\left(\mathrm{C}_{\mathrm{L}}\right)_{\text {target }}$ & 0.247 \\
\hline
\end{tabular}




\section{Chapter 6: Results - Performance Analysis}

The six different flap configurations were analyzed at the various flight conditions and flap settings to map the performance and aerodynamic characteristics of their respective geometries. Throughout the simulations the aerodynamic coefficients of $\mathrm{C}_{1}$,

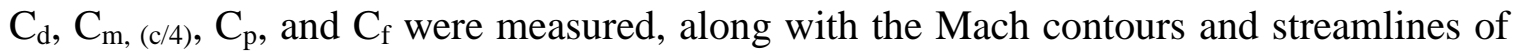
each converged solution. Each of the flap configurations for the low airspeed cases, the $60^{\circ}$ and $90^{\circ}$ deflections, were evaluated at three different $\alpha$ 's of $-5^{\circ}, 0^{\circ}$, and $3^{\circ}$. The cruise case configurations were only analyzed at the specific $\alpha$ that produced the target cruise $C_{1}$ of the AMELIA design, which corresponded to slightly different $\alpha$ 's for each flap configuration.

\section{Takeoff Performance - Low Airspeed, $60^{\circ}$ Flaps}

Table 6-1 below presents Mach contours and streamlines for all six flap configurations at $\alpha=0^{\circ}$ and the takeoff conditions and settings. For the Mach and streamline results of the other $\alpha$-values that were simulated during the analysis, refer to the appendix. 
Table 6-1: $60^{\circ}$ flap streamlines and contours of Mach at $\alpha=0^{\circ}$

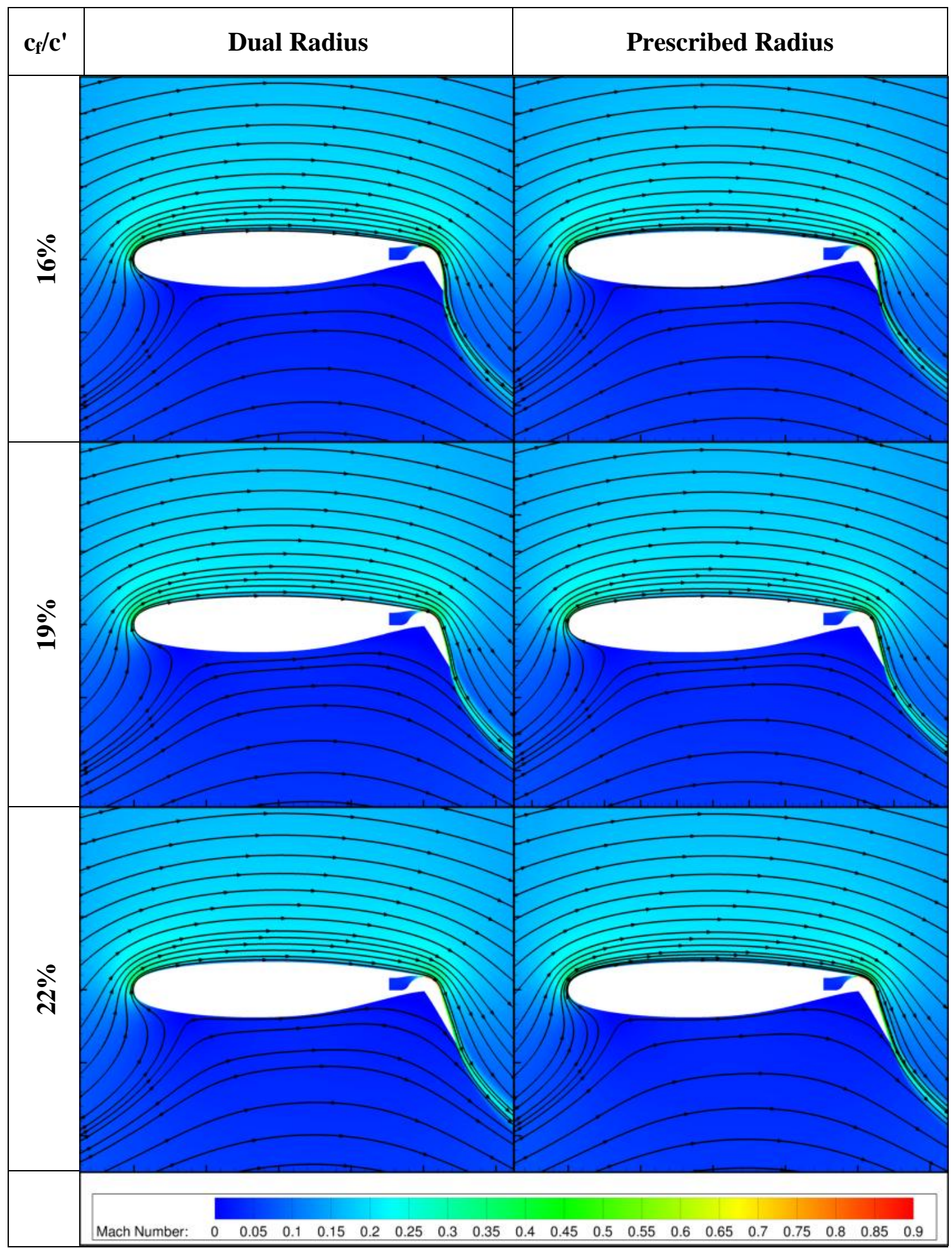


Note the increased regions of velocity near the leading edge and above the trailing edge slot on each airfoil, and significant streamline curvature. Throughout all takeoff simulations no sonic flow was present in any of the computational domains. Several points can be taken from the flow visualization of the simulation, presented in Table 6-1. The first being the difference in stagnation point location, not only for the different length in flaps but also the dual radius versus the prescribed radius configurations. The location of the stagnation points for the different configurations indicate the effect each have on their ability to augment forces, with the further aft the stagnation location the greater the augmentation. For the dual radius configurations the lengthening of the flap brought the stagnation point further forward on the lower surface of the airfoil, where the length of prescribed radius flaps had little influence on the stagnation point location (note the similar locations of PR16 and PR22). All of the dual radius flap configurations encountered noticeably further aft stagnation locations than the corresponding prescribed radius flaps, with the difference lessening as flap length increased. Looking at the aft region of the flow visualization, it is noted that the longer flaps with smaller $\theta$ allowed the aft streamlines to smoothly transition over the trailing edge of the flap surface rather than experience the much more abrupt and rapid turns of the shorter flaps. From the observations above, both the shorter flaps and the dual radius flaps had increased effectiveness on force augmentation indicating more lift was being produced as well as the accompanying drag and negative moment (nose down) forces.

\section{Lift, Drag, and Moment Data}

The analytical plots of $C_{1}$ vs. $\alpha$ and $C_{d}$ vs. $\alpha$ are displayed in Figure 6-1 and Figure 6-2, respectively. 


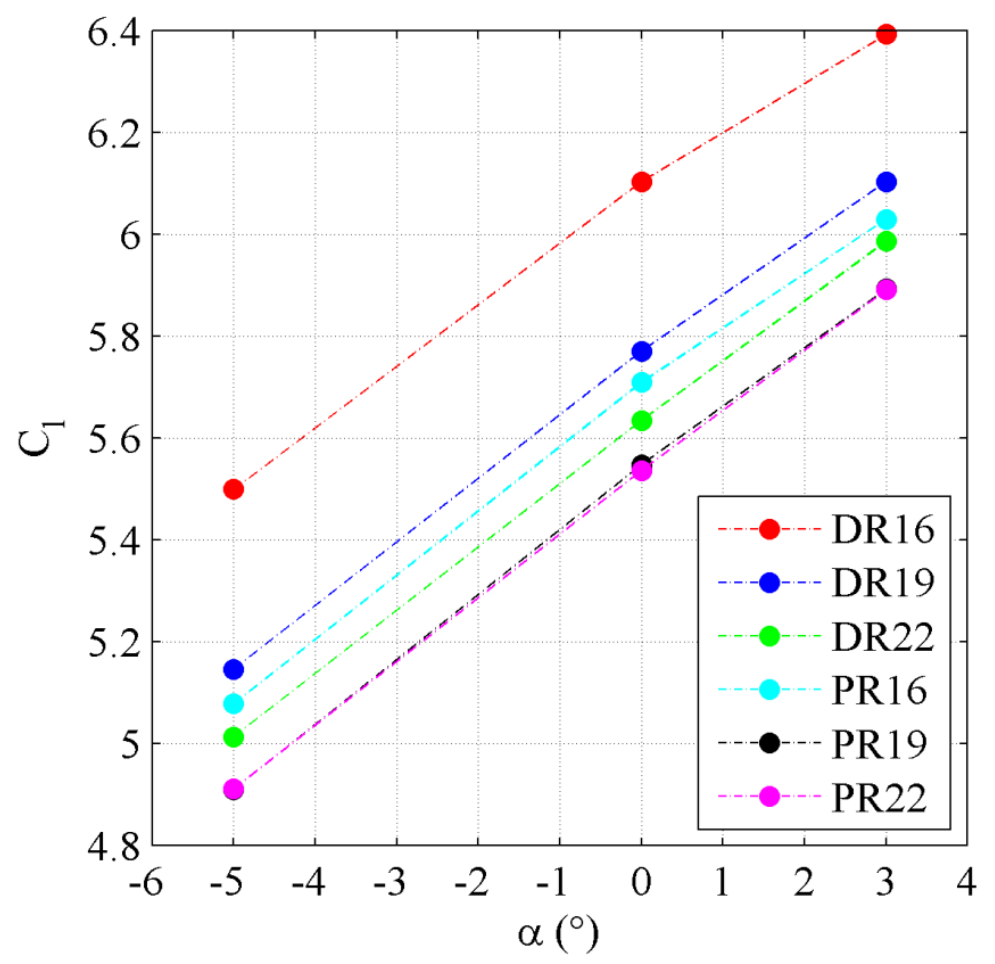

Figure 6-1: Lift for $60^{\circ}$ flap configurations

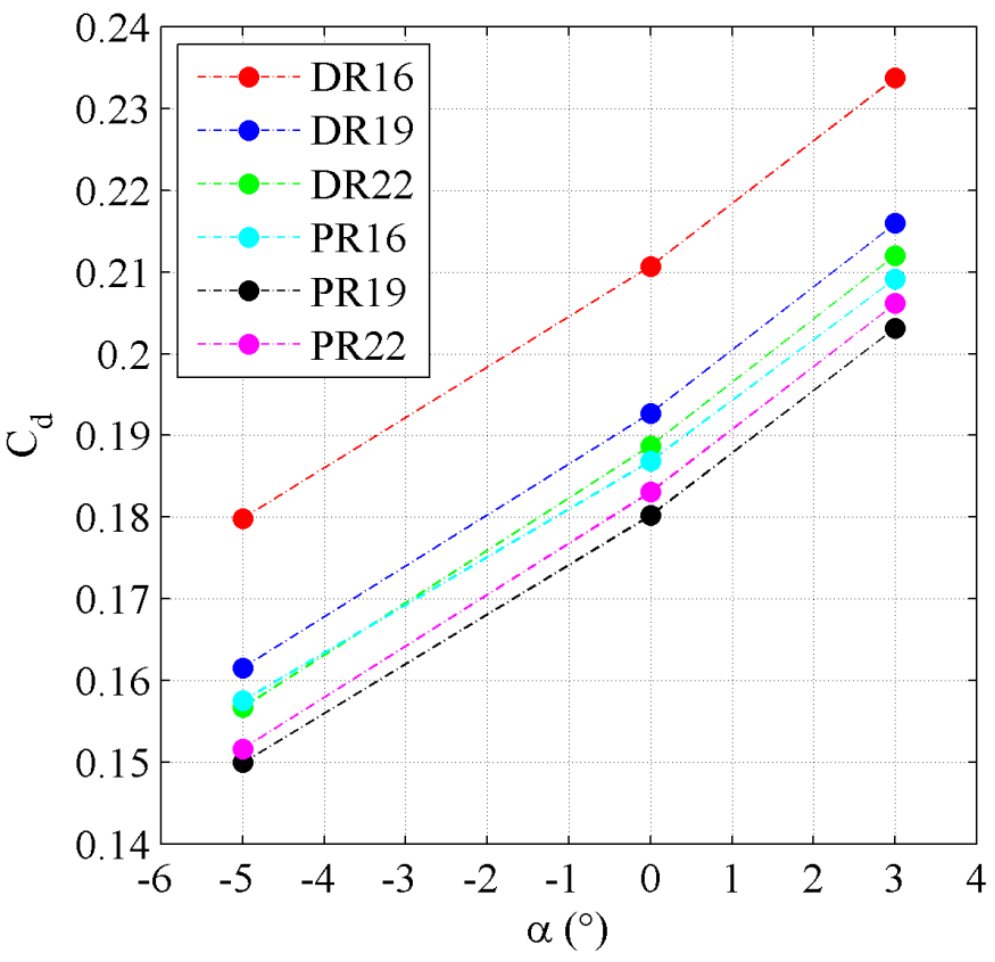

Figure 6-2: Drag for $60^{\circ}$ flap configurations 
From Figure 6-1 above, all the flap configurations experienced an approximately linear trend as $\alpha$ is increased having a consistent difference between each configuration over all $\alpha$ 's examined, as well as showing that all configurations have yet to reach their respective stall $\alpha$ 's. It is clear that configuration DR16 achieved the greatest lift augmentation for the $60^{\circ}$ deflection, reaching a $C_{1}$ of 6.4 at $\alpha=3^{\circ}$. The lift curve for DR19 dropped off significantly from DR16, having a 5.5\% average drop in lift. The reason for this significant variation in lift between DR16 and the other flaps was attributed to the larger departure angle $(\theta)$ encountered on DR16 with the smallest radius at the trailing edge of the flap. DR19 slightly outperformed PR16, having only a $1.2 \%$ average increase in lift. Though this increase in lift of DR19 over PR16 cannot be attributed to $\theta$, due to PR16 having a slightly larger $\theta$ of $23.92^{\circ}$ compared to the $21.22^{\circ}$ of DR19. Instead these additional lift gains were credited to the $3 \%$ increase in flap length. The similar lift-performance of PR19 and PR22 was assumed to be due to the additional $3 \%$ length of flap in the PR22 making up for the smaller $\theta$ angle and less surface curvature of PR22 and yielding approximately the same lift.

The drag data displayed in Figure 6-2 revealed that all of the drag trends closely followed the trends observed in each configurations lift curves. This resulted in higher drag values produced by the configurations that augmented the most lift, with DR16 generating significantly more drag than the other configurations. The only differences between the lift and drag trends were DR22 producing more drag (and less lift) than PR16 and PR19 achieving the lowest drag. With additional observation of the flap specifications (Table 4-1), it showed that a lower drag value can be achieved with a larger $\theta$-value by using the different variation in surface curvature of the prescribed radius and 
shorter length while producing slightly more lift. This contradicted what was previously found in the preliminary study on the various dual radius configurations with the PR16 having a lower $r_{2 \_a v g} / r_{1}$-value (30.7) compared to DR22's $r_{2} / r_{1}$-value (48), but again was attributed to the different geometrical shape of the prescribed radius.

To more directly compare the $\mathrm{C}_{1}$ and $\mathrm{C}_{\mathrm{d}}$ values with each other and $\alpha$, the plots of $C_{d}$ vs. $C_{1}$ and L/D vs. $\alpha$ were constructed and presented below in Figure 6-3 and Figure 6-4.

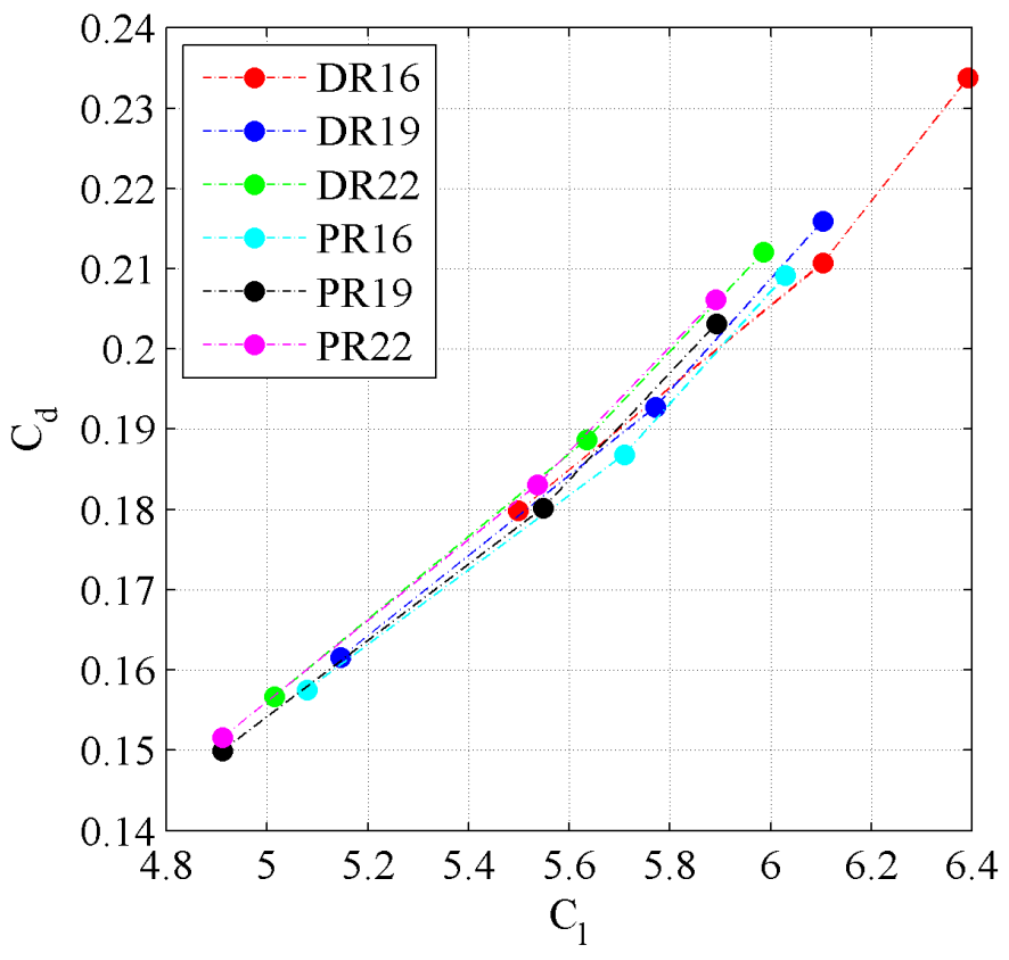

Figure 6-3: Drag given lift for $60^{\circ}$ flap configurations 


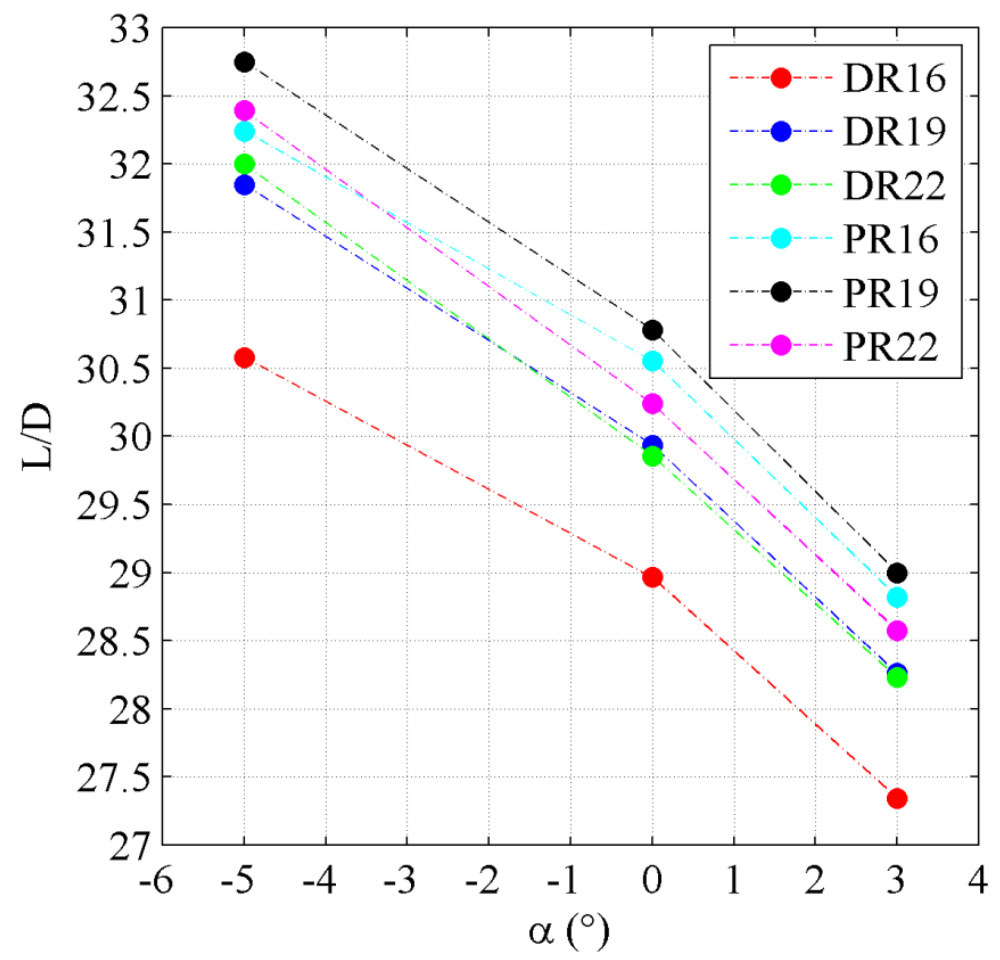

Figure 6-4: Lift-to-drag ratios for $60^{\circ}$ flap configurations

From the $\mathrm{C}_{\mathrm{d}}$ vs. $\mathrm{C}_{1}$ plot above it can be seen that all of the $\mathrm{CC}$ flap configurations had very similar drag-to-lift trends for the $\alpha$-values investigated. Since the flap deflection of $60^{\circ}$ was intended for takeoff operations in this study, the L/D vs. $\alpha$ plot provided greater insight on which configurations were best suited for takeoff performance. The L/D plot clearly highlights the PR19 configuration having the best L/D ratio over the other configurations for the range of $\alpha$ 's analyzed, which climbed up to an L/D of 32.8 at $\alpha=-5^{\circ}$ and had an average L/D of 30.8. All of the prescribed radius configurations experienced better L/D performance than the dual radius flaps, suggesting the varying surface curvature of the prescribed radius can produce the same lift with less drag penalties of the constant curvature of the dual radius. Another interesting note is how all flap configurations show that their max L/D occur below $\alpha=-5^{\circ}$, which is caused by the severe increase in circulation from the CC flap system. 
Figure 6-5 displays additional L/D data and Figure 6-6 displays $\mathrm{M}_{(\mathrm{c} / 4)}$ data below.

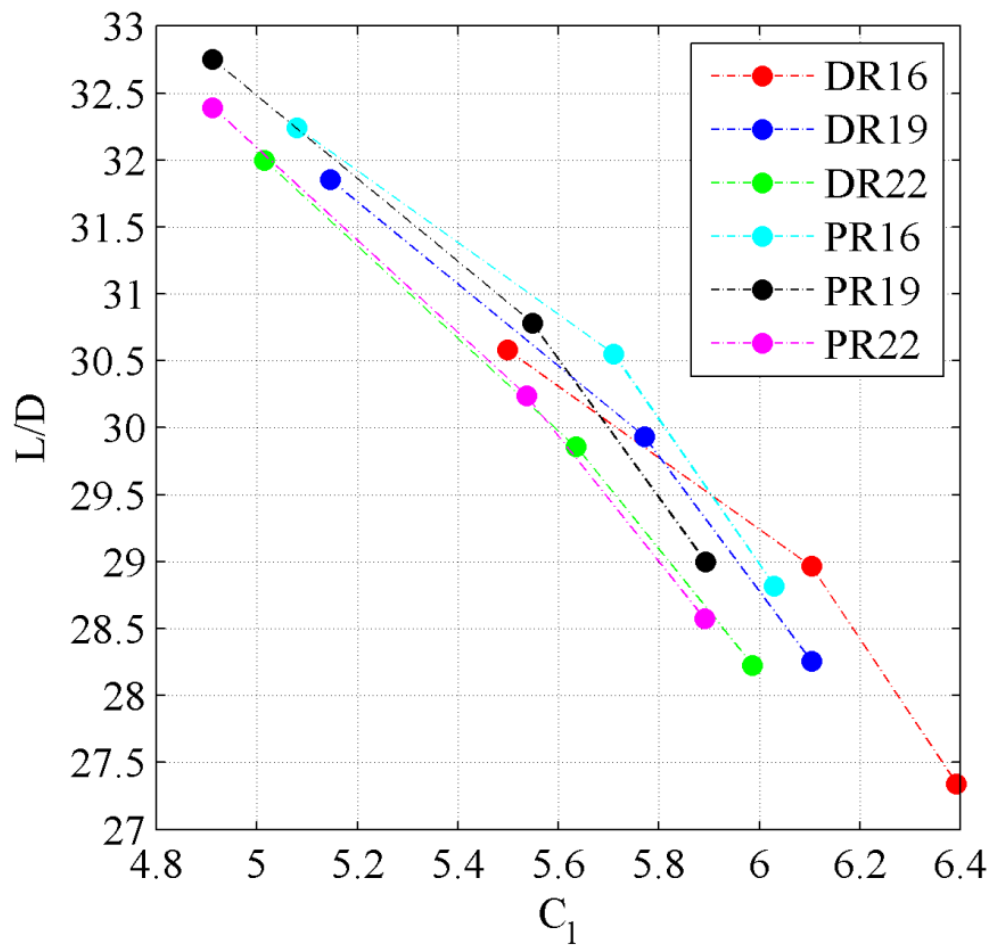

Figure 6-5: Lift-to-drag ratios given lift for $60^{\circ}$ flap configurations

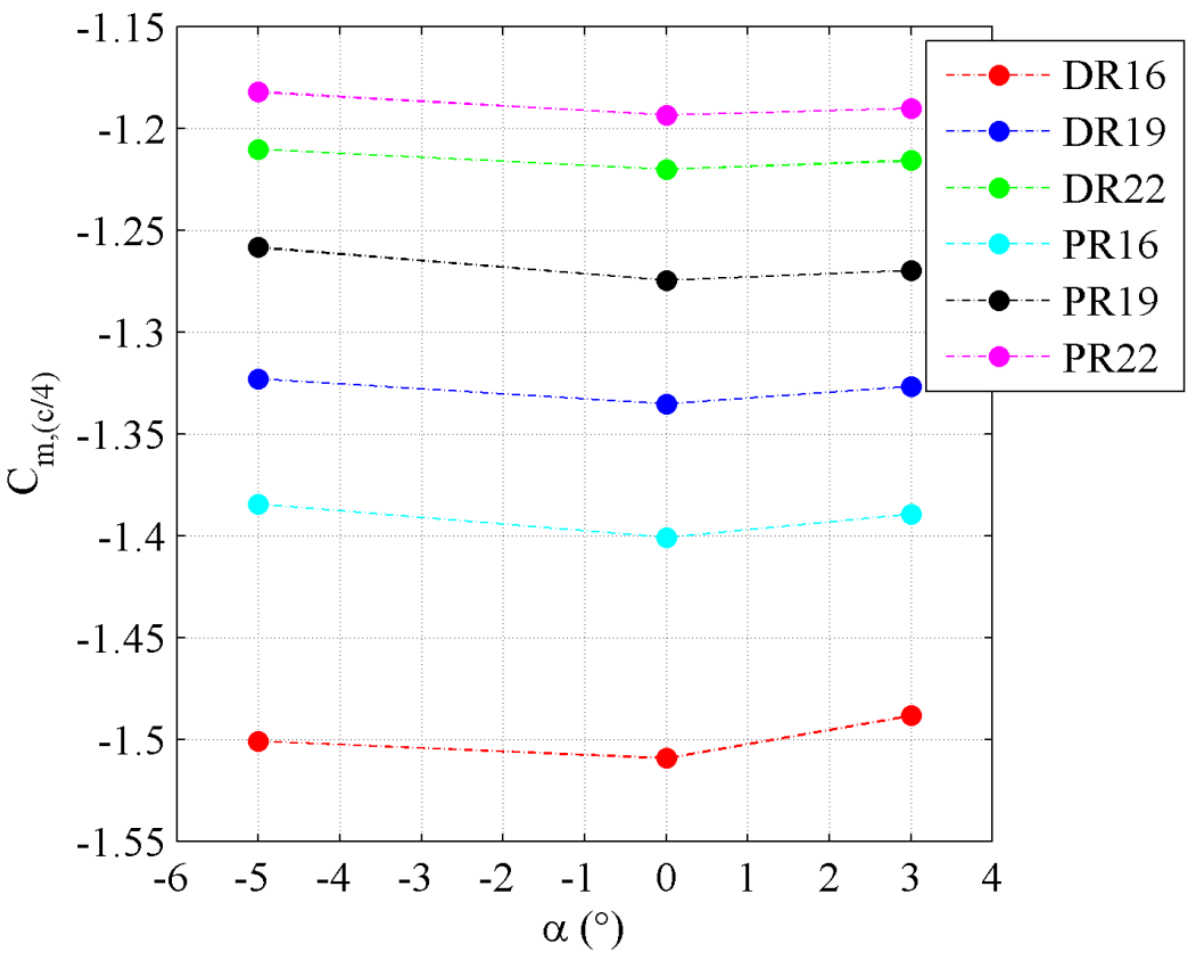

Figure 6-6: Pitching moment for $60^{\circ}$ flap configurations 
The $\mathrm{L} / \mathrm{D}$ ratios given lift generated data again shows the benefit of the prescribed radius configurations, reaching larger L/D ratios. All flap configurations show the same trend in decreasing $\mathrm{L} / \mathrm{D}$ ratios with increase in lift, with the $\mathrm{L} / \mathrm{D}$ dropping at a greater rate for the higher lift values. Given a specific $\mathrm{C}_{1}$ the $16 \%$ and $19 \%$ prescribed radius flaps achieved the higher L/D ratios, showing an advantage over the $22 \%$ flaps and all the dual radius flaps.

The $\mathrm{C}_{\mathrm{m} \text {, (c/4) }}$ vs. $\alpha$ plot above shows the large negative moments encountered by all the CC flap configurations, which has been observed in past CC research. ${ }^{1,2,5,9,15,20,38,47,49,62,102}$ DR16 experienced the worst $C_{m}$, (c/4) of -1.5 , which can be directly attributed to its large lift augmentation and significant $\theta$-value of $33.12^{\circ}$. PR16 followed behind DR16's large negative quarter-chord pitching moment with a 7.2\% decrease in pitching moment. Looking at both dual radius and prescribed radius results separately, they experienced a similar relationship with the length of the flap and the magnitude of the negative quarter-chord moment with the longest flaps encountering the least moment. Although both configurations experienced similar trends with length of flap, the prescribed radius configurations stayed consistently below the magnitude of moment created by the dual radius flaps of the same chord length.

\section{Pressure and Skin Friction Data}

A more detailed analysis of the different $60^{\circ}$ configurations was pursued by plotting $C_{p}$ vs. $x / c^{\prime}$ for the $\alpha=0^{\circ}$ cases, which is displayed below in Figure 6-7. The red vertical lines in the following figures represent the slot location of each airfoil. 


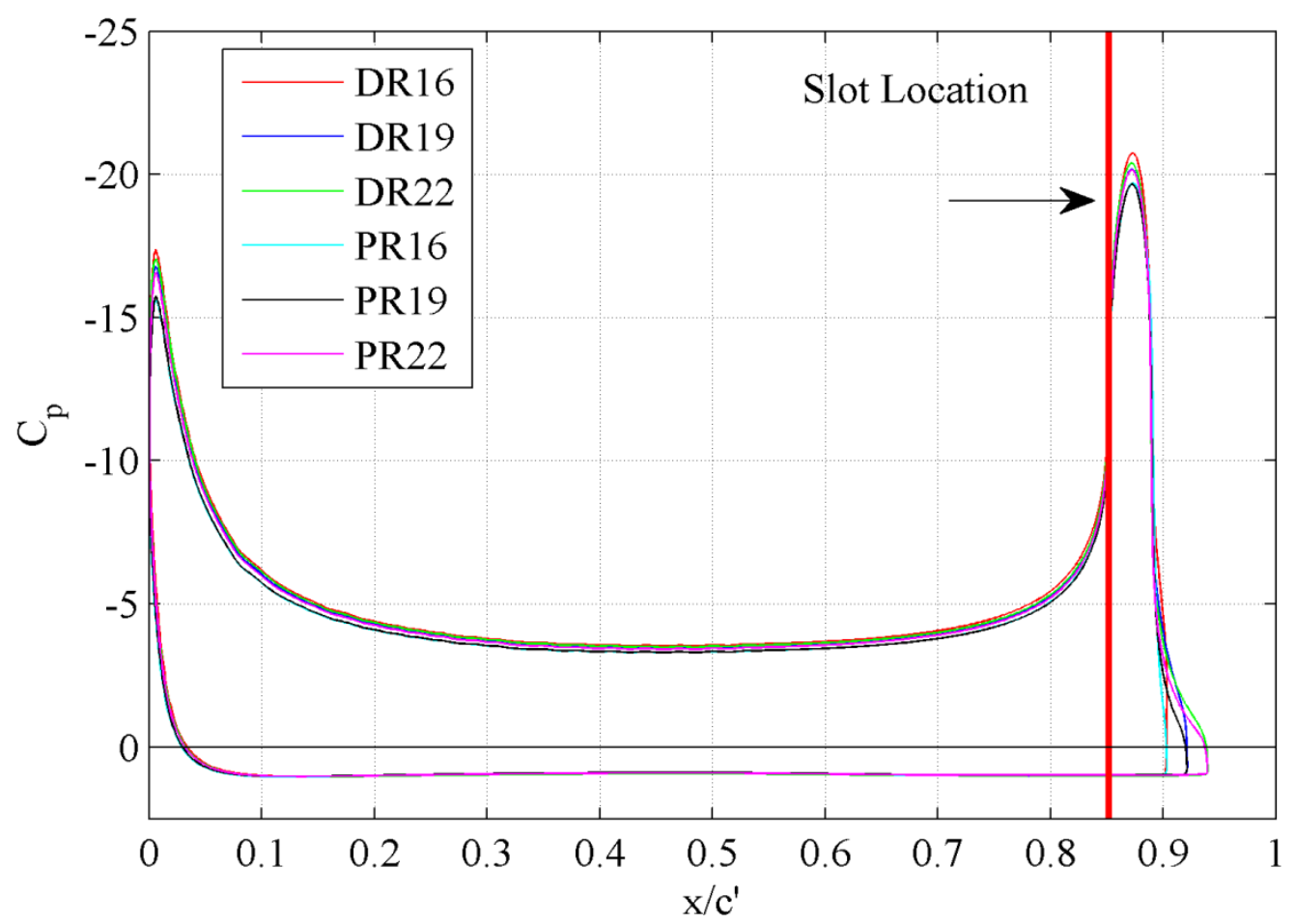

Figure 6-7: Pressure distributions for $\alpha=0^{\circ}, 60^{\circ}$ flaps

From the $\mathrm{C}_{\mathrm{p}}$ plot above, all configurations experienced similar pressure distributions over the airfoil with a large suction peak near the leading edge (-16.5) and even bigger peak occurring over the blown flap surface (-20), which are consistent with pressure distributions of past research. ${ }^{12,52}$ This larger pressure peak of the aft region of the airfoil is caused by the CC blowing performed on the trailing edge of the airfoil and gives the airfoils the large associated negative pitching moment. It is evident that the different configurations varied the most significantly in the regions of the suction peaks, as well as the longer flaps that extended negative $\mathrm{C}_{\mathrm{p}}$-values further aft in the $\mathrm{x}$-direction. Another observation, would be the sudden drop in $\mathrm{C}_{\mathrm{p}}$, or sudden flow deceleration, around $x / c^{\prime}$ of 0.89 . This location corresponds with the change in flap surface, from the constant arc $r_{1}$ to the secondary flap surface determined by each flap configuration for the $60^{\circ}$ deflection. All of the configurations experienced this adverse pressure gradient, 
which could be problematic in causing upper surface separation for lower $\mathrm{C}_{\mu}$ cases. To more closely examine the variations in suction peaks between the configurations, a zoomed in view of both leading edge and trailing edge peaks were produced and are presented below in Figure 6-8 and Figure 6-9.

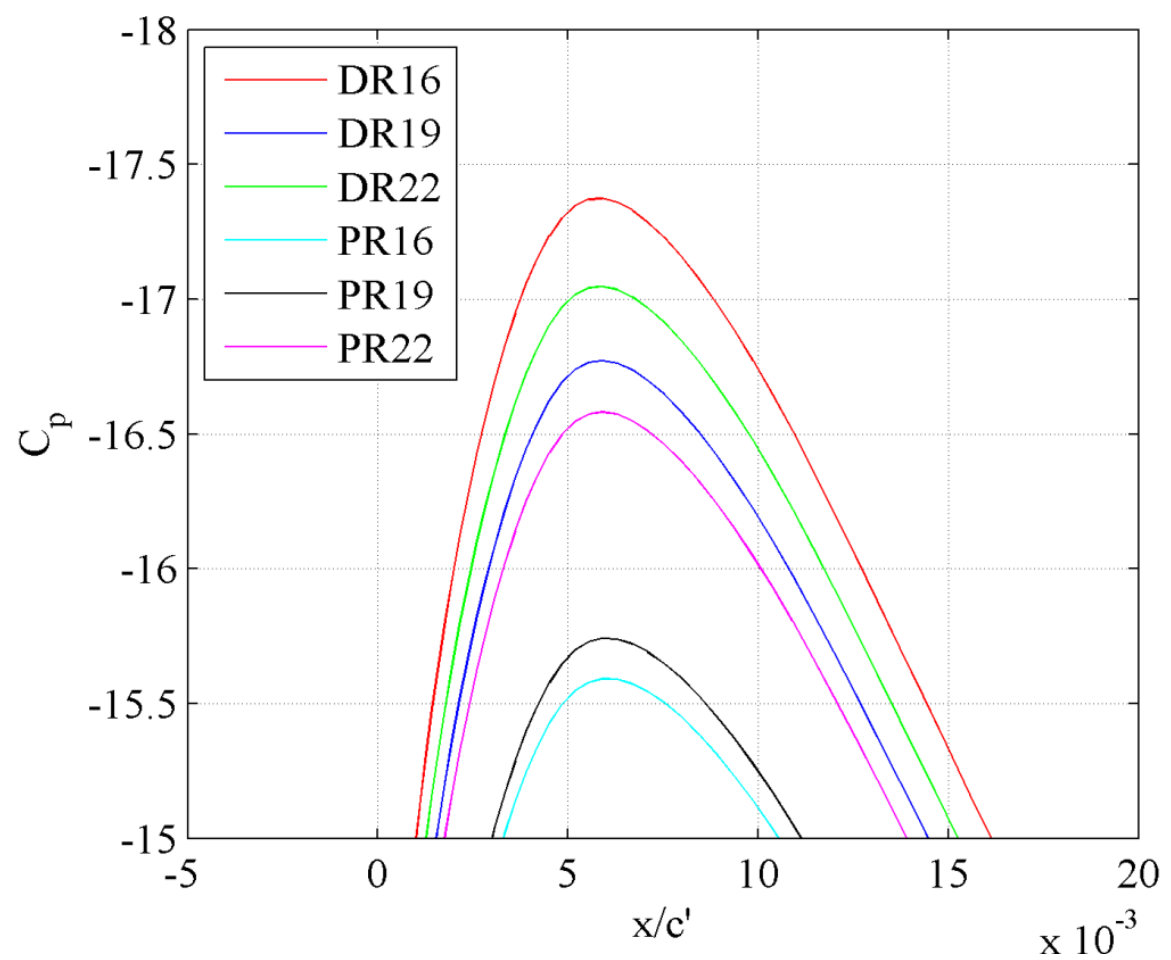

Figure 6-8: Leading edge suction peaks, $\alpha=0^{\circ}, 60^{\circ}$ flaps 


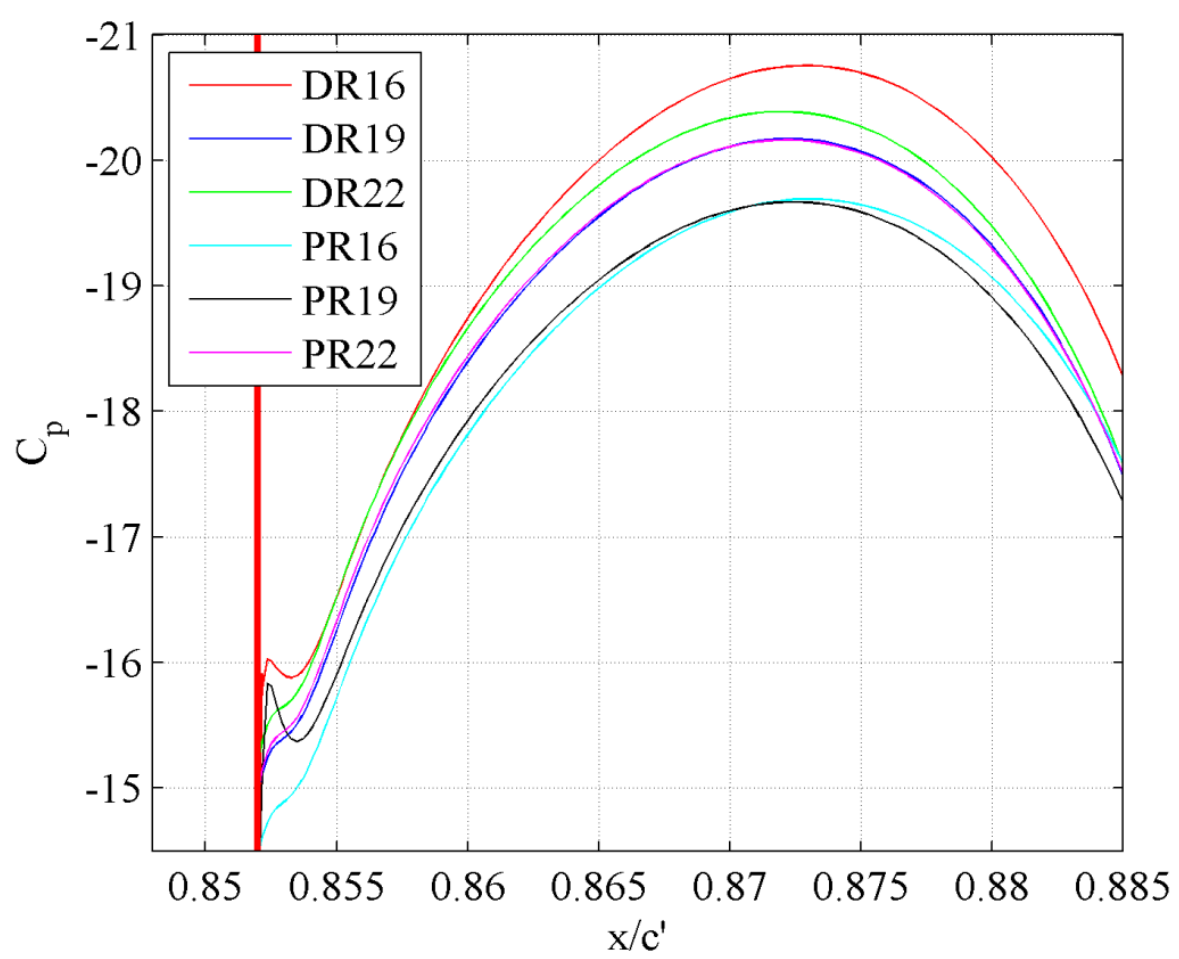

Figure 6-9: Trailing edge suction peaks, $\alpha=0^{\circ}, 60^{\circ}$ flaps

The leading edge suction peak plot displays all three dual radius configurations were superior in obtaining a more negative pressure peak, with DR16 achieving the highest peak at -17.4 and DR22 following at -17.1. PR22 generates the most negative pressure peak of the prescribed radius flaps at -16.6, falling just shy of the peak of DR19 at -16.8. Figure 6-9 displays very similar trends of the different configurations on the trailing edge suction peak, with DR16 and DR22 achieving more negative pressure peaks (-20.8 and -20.4) than any of the prescribed radius flaps, and DR19 having about the same pressure peak as the best performing prescribed radius configuration of PR22 at 20.2. These pressure plots reveal the dual radius configurations attain more negative pressure peaks than the prescribed radius flaps. Another observation was that there was a sharp acceleration then deceleration in the flow directly aft of the slot experienced by all of the flaps, shown by the slight decrease in slope of $\mathrm{C}_{\mathrm{p}}$ just aft of the red slot location 
line in Figure 6-9. The source of this is believed to be caused by the expansion of the slot flow effective area just aft of the slot which accelerates the local flow velocity. This acceleration of the slot flow over the beginning of the flap surface is depicted in Figure 6-10 below.

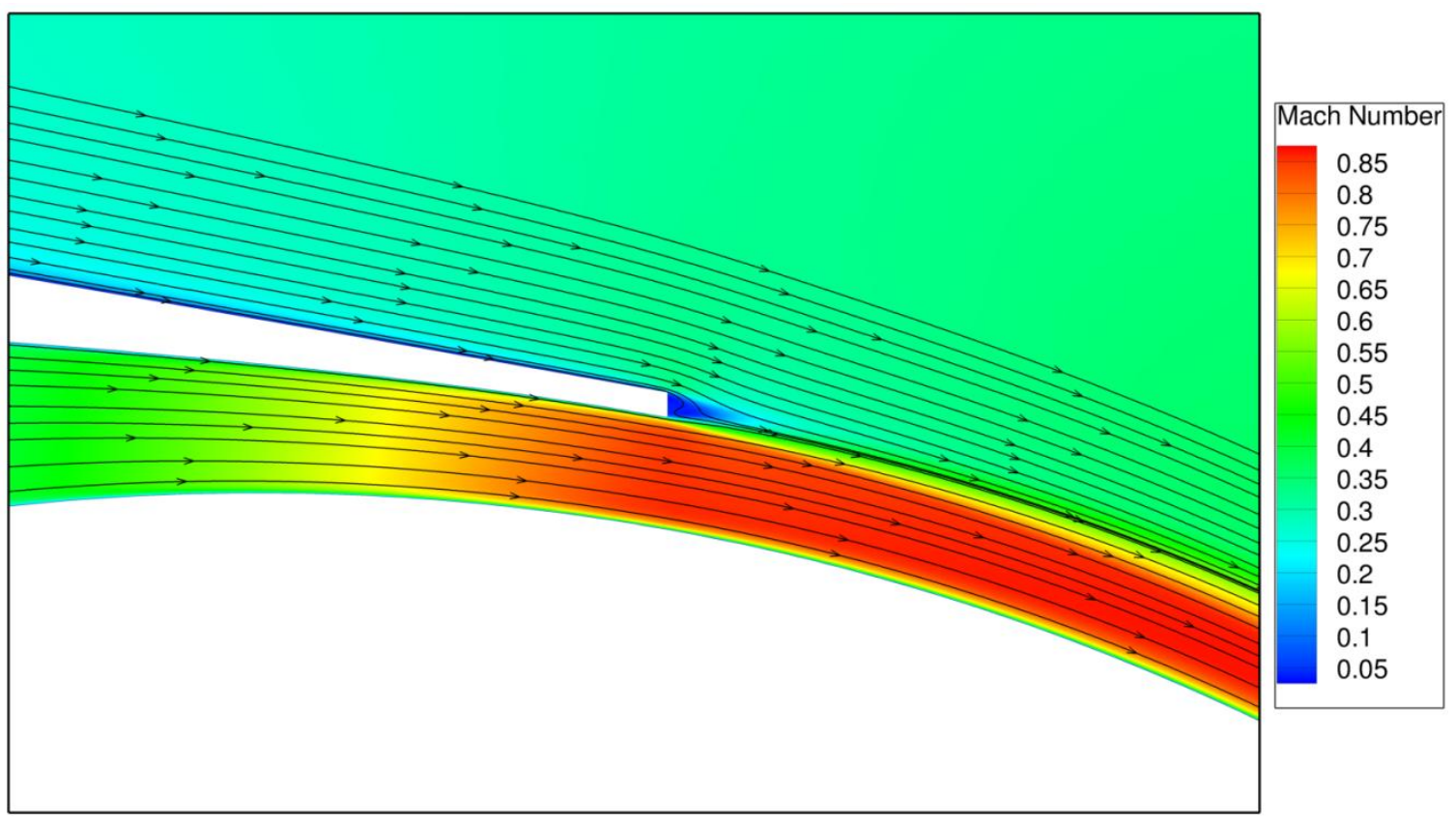

Figure 6-10: Acceleration of slot flow on flap surface

Figure 6-11, below, depicts the $C_{p}$ values acting directly on the flap surface. This clearly shows that all three dual radius flaps carried a more negative $\mathrm{C}_{\mathrm{p}}$ further aft on the flap surface when compared to the prescribed radius flaps. This is a contributor to the dual radius encountering larger lift augmentation and negative pitch down moments when compared to the same length prescribed radius configuration. 


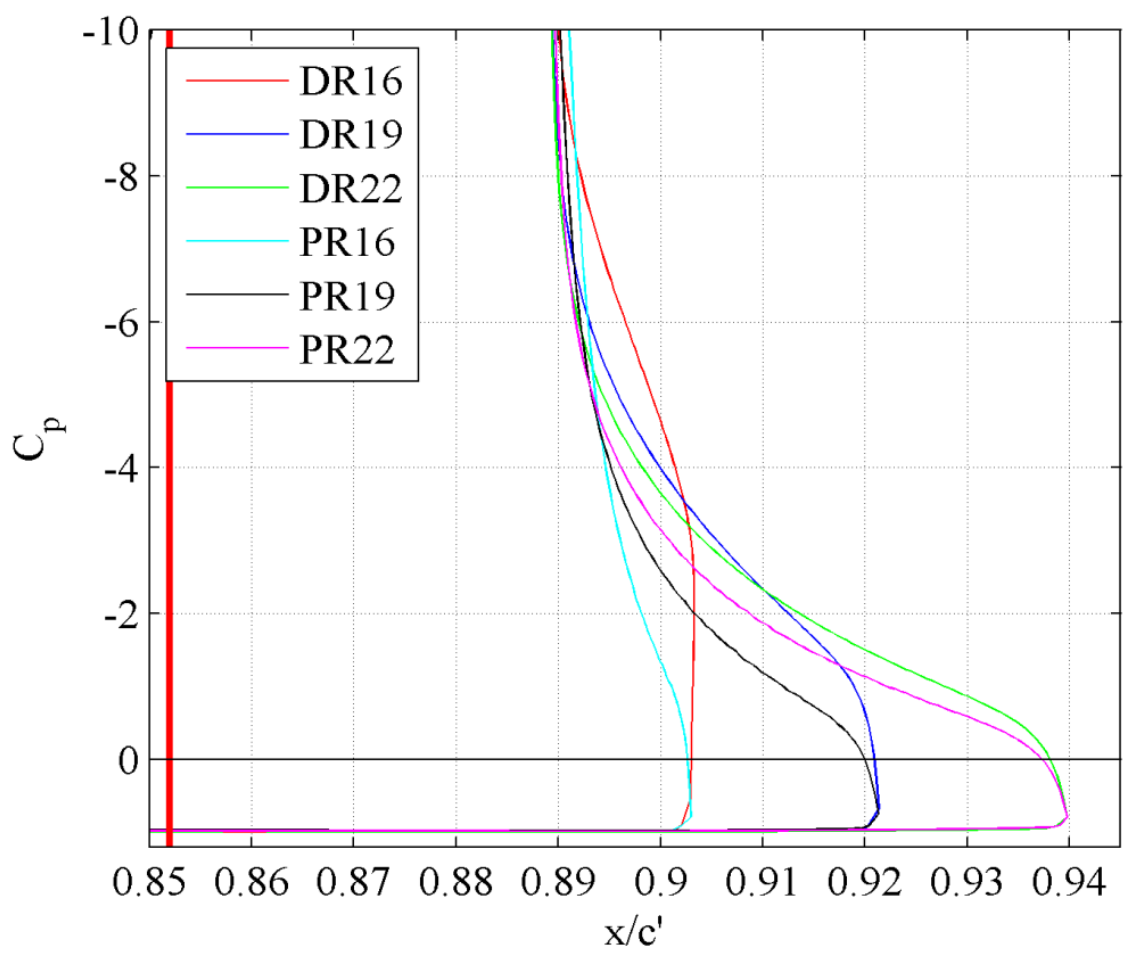

Figure 6-11: Flap surface pressure distributions, $\alpha=0^{\circ}, 60^{\circ}$ flaps

The $\mathrm{C}_{\mathrm{p}}$ plots for $\alpha=-5^{\circ}$ and $\alpha=3^{\circ} 60^{\circ}$ flap configurations are presented below in Figure 6-12 and Figure 6-13 respectively. 


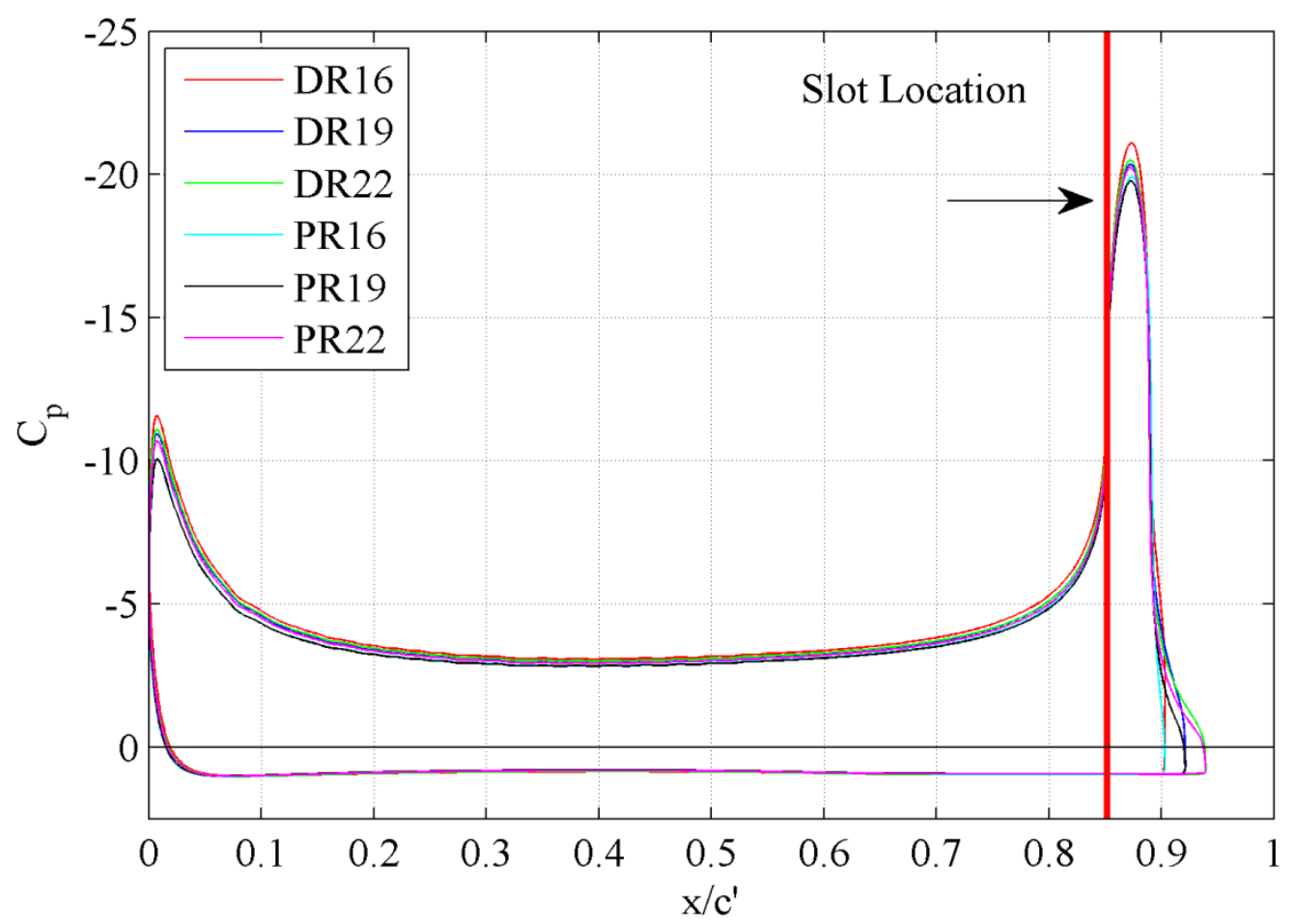

Figure 6-12: Pressure distributions for $\alpha=-5^{\circ}, \mathbf{6 0}^{\circ}$ flaps

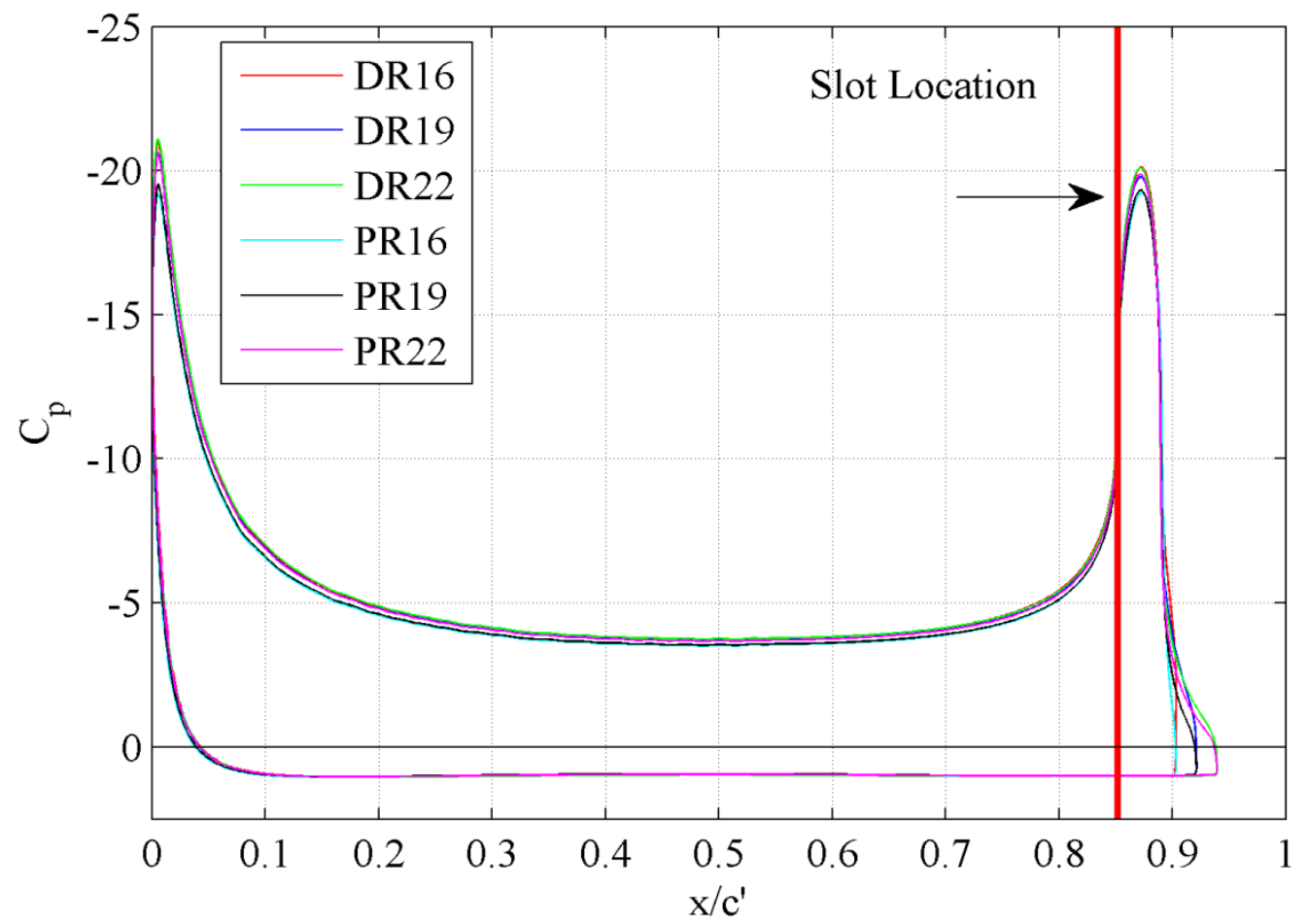

Figure 6-13: Pressure distributions for $\alpha=3^{\circ}, 60^{\circ}$ flaps 
The $\alpha=-5^{\circ}$ plot of $C_{p}$ shows the constant smaller values carried over the leading edge and majority of the upper surface of the airfoil compared to the $\alpha=0^{\circ} \mathrm{C}_{\mathrm{p}}$ plot. The leading edge suction peak now only climbs to a $C_{p}$ of approximately -11 rather than the 16 of the $\alpha=0^{\circ}$ case. Although, when comparing the aft suction peaks in the region of the slot, both $-5^{\circ}$ and $0^{\circ}$ cases, they appear very similar both averaging $C_{p}$ values around 20. Each of the flap configurations keep their same order of performance, with DR16 experiencing the largest suction peaks in the regions of the leading edge and slot location.

The $\alpha=3^{\circ} \mathrm{C}_{\mathrm{p}}$ plot showed larger leading edge suction peaks with an average $\mathrm{C}_{\mathrm{p}}$ of -20 , but did not have a larger $C_{p}$ over the majority of the airfoil when compared to the $\alpha=0^{\circ}$ case. The larger leading edge pressure peaks of the $\alpha=3^{\circ}$ condition are caused by the increased airfoil incidence which forces the airflow to accelerate even faster around the elevated leading edge. Again, the trailing edge stayed at the average $C_{p}$ of -20 that both $\alpha=-5^{\circ}$ and $\alpha=0^{\circ}$ conditions encountered. Though at the $\alpha=3^{\circ}$ condition saw DR16 fall below the leading edge and slot region suction peaks of DR22, shown in below Figure 6-14 and Figure 6-15 respectively. This shows the start of performance degradation due to the flap and slot flow not being able to influence the upper airfoil flow downward as efficiently. At the $3^{\circ}$ of $\alpha$ and given $C_{\mu}$ value of 0.3 , the DR16 flap begins the experience the onset of stall. When looking back at the lift data (Figure 6-1), it can be more clearly seen that the $\alpha=3^{\circ}$ data point of DR16 does not continue the linear relationship experienced at lower $\alpha$ values and by the other flap configurations. 


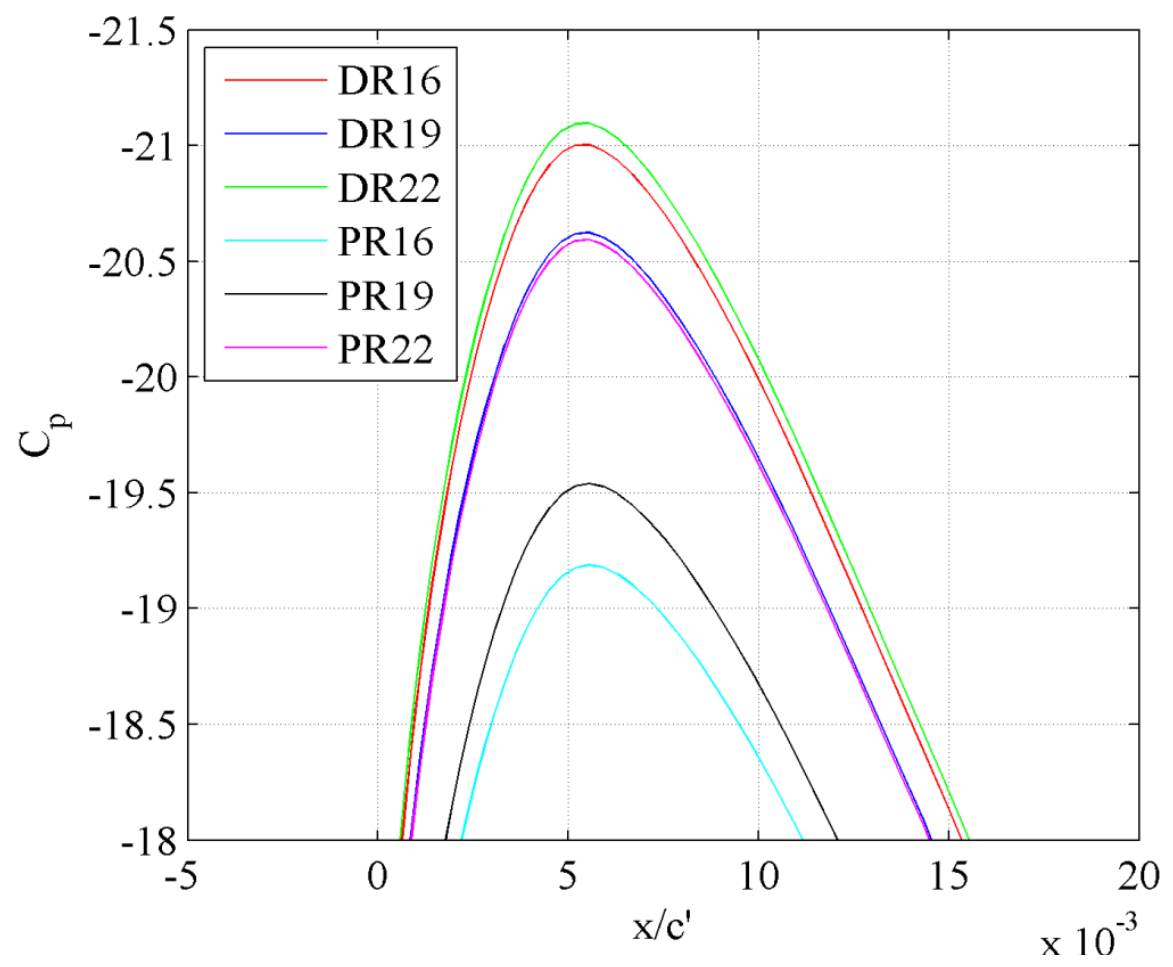

Figure 6-14: Leading edge suction peaks, $\alpha=3^{\circ}, 60^{\circ}$ flaps

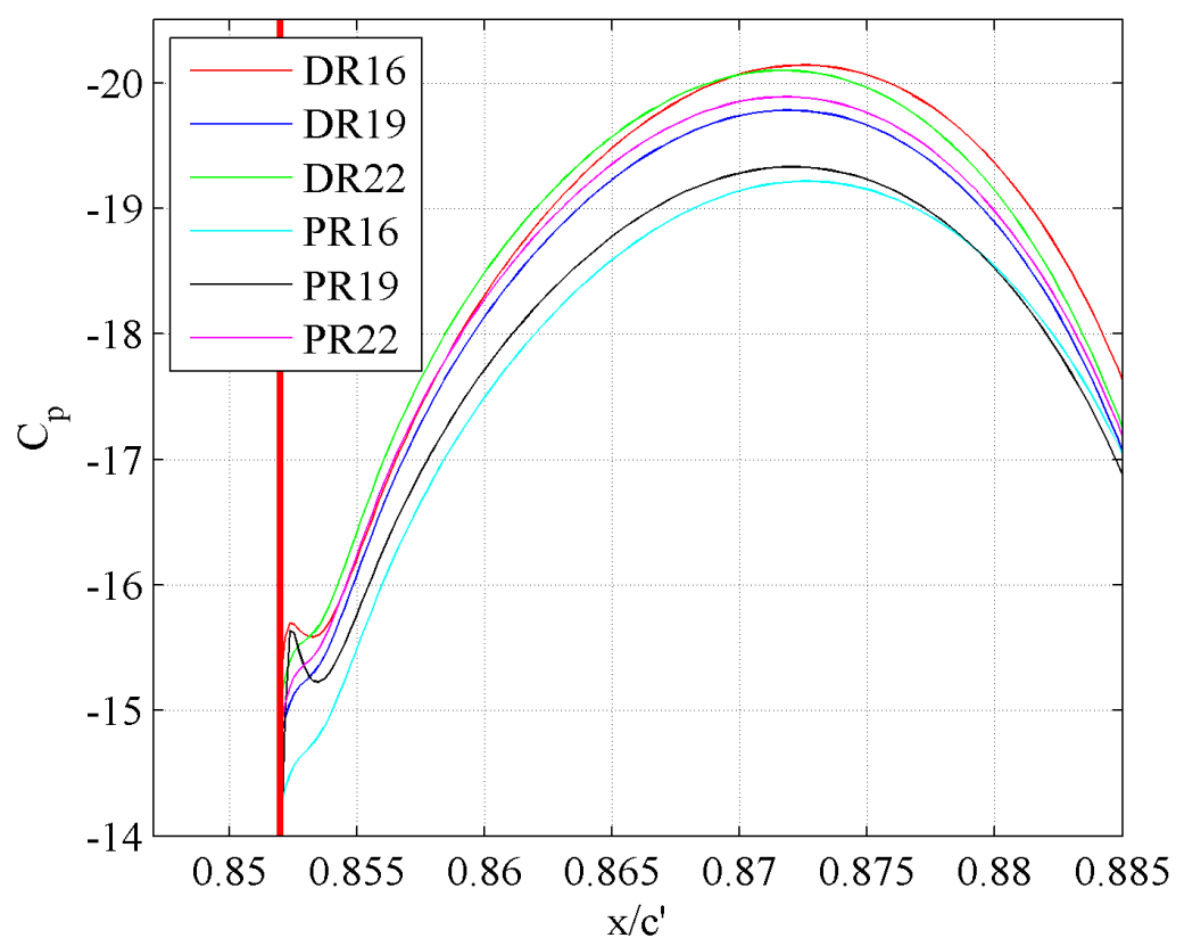

Figure 6-15: Trailing edge suction peaks, $\alpha=3^{\circ}, 60^{\circ}$ flaps 
The resulting plot of $\mathrm{C}_{\mathrm{f}}$ for each of the airfoils at $\alpha=0^{\circ}$ is shown below in Figure 6-16 for further analysis.

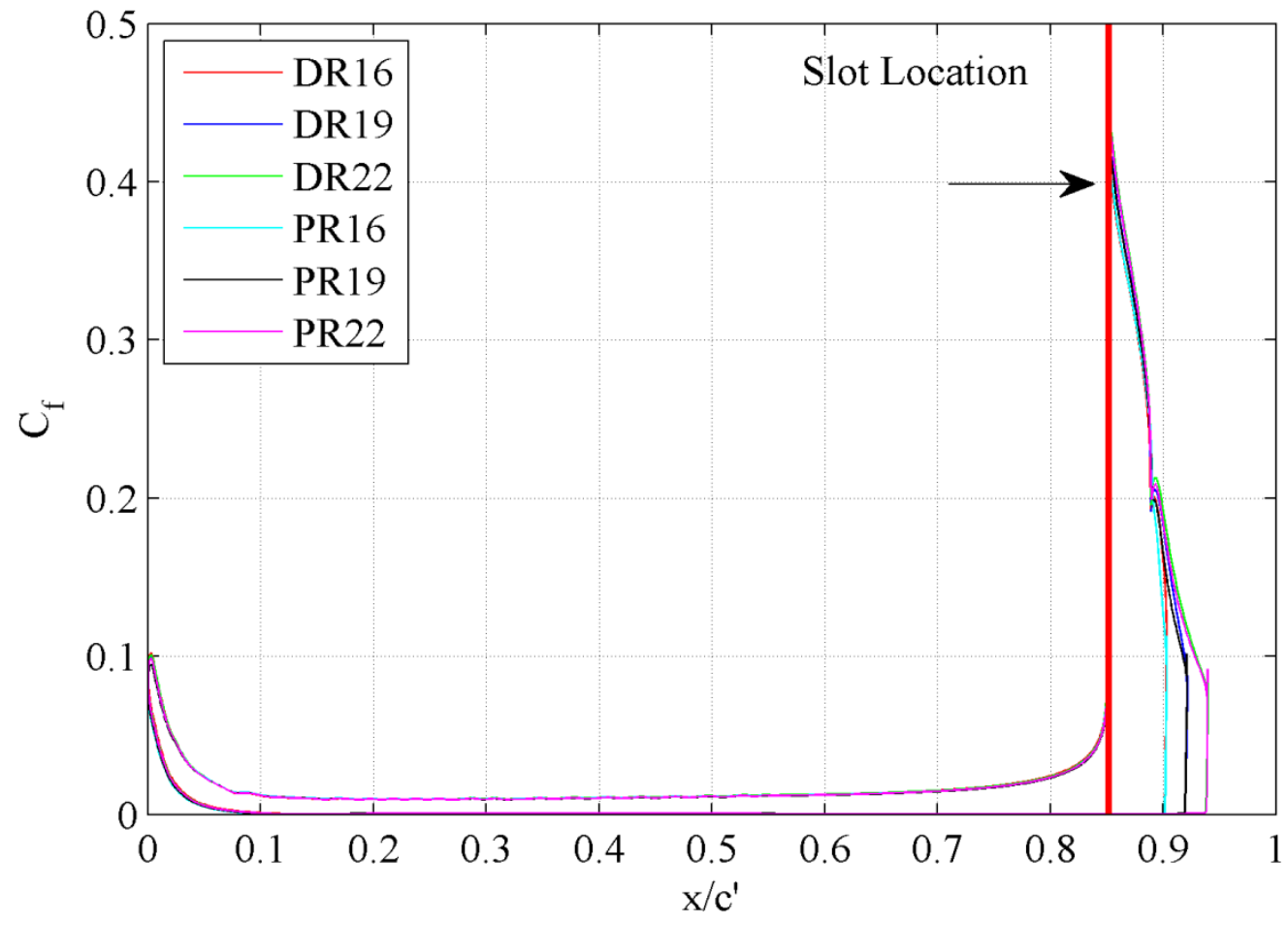

Figure 6-16: Skin friction distributions for $\alpha=0^{\circ}, 60^{\circ}$ flaps

This plot provides the general skin friction encountered during CC operation, with slot location given with vertical red line. Forward of the slot all of the flap configurations behaved very similar, with the leading edge peaks vary slightly between the configurations, occurring in the same order as each flap's corresponding $C_{p}$ value from Figure 6-8 above. The main variation of $\mathrm{C}_{\mathrm{f}}$ between the flaps took place aft of the slot, where the influences of the geometry of each of the configurations came into play. Figure 6-17 below details $C_{f}$ values in the region aft of the slot which is where the surface of each configuration varies. 


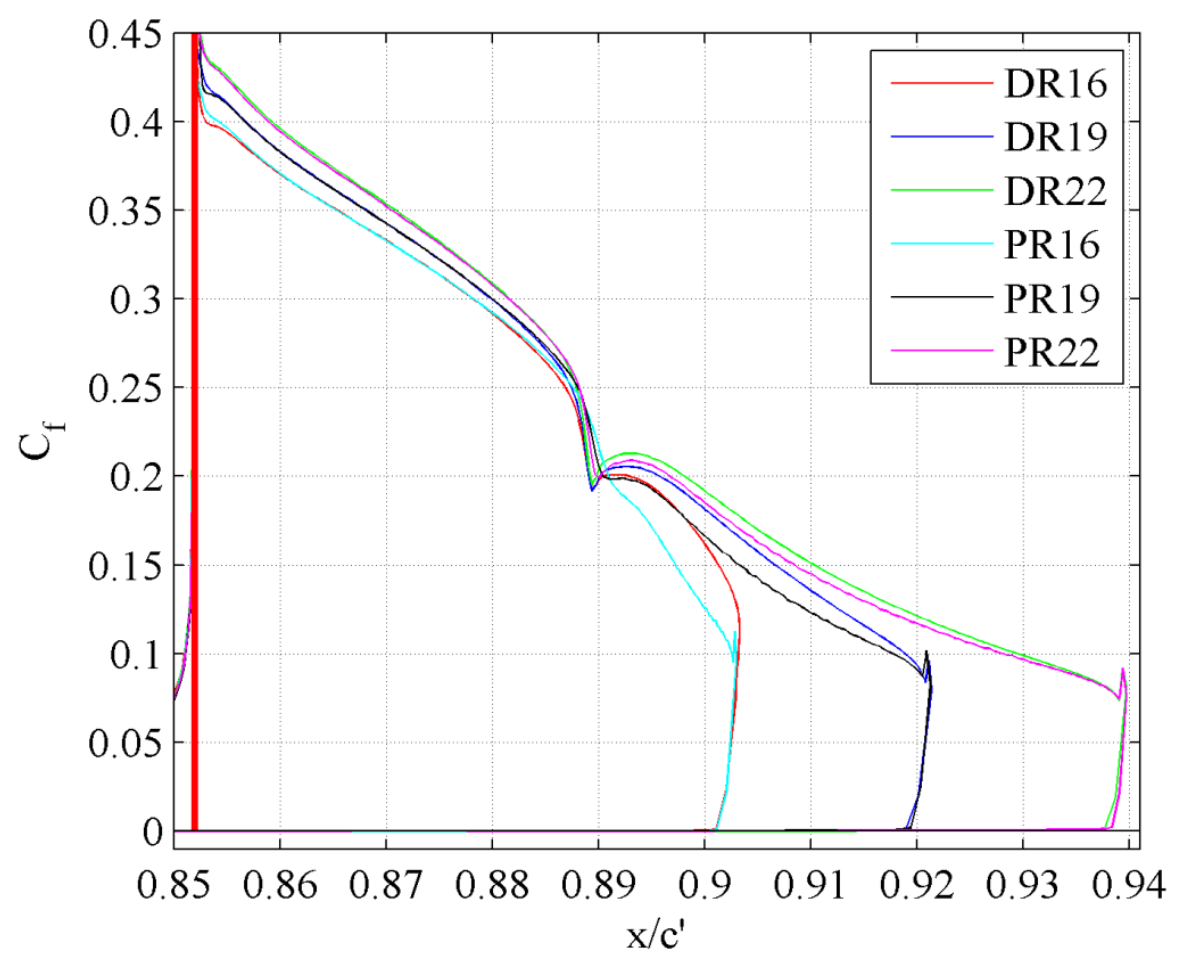

Figure 6-17: Flap surface skin friction distributions, $\alpha=0^{\circ}, 60^{\circ}$ flaps

All of the configurations encountered a small decrease in $\mathrm{C}_{\mathrm{f}}$ shortly aft of the slot, which is again assumed to be caused by the expansion of the slot flow effective area just aft of the slot accelerating then decelerating the flow velocity along the curved flap surface (Figure 6-10). When inspecting this aft region of the airfoil, it can be seen that the length of each of the flaps had the most effect on the $\mathrm{C}_{\mathrm{f}}$ value. The longer the flap length, the larger the $\mathrm{C}_{\mathrm{f}}$ value for the duration of the flap. When comparing the same length dual radius and prescribed radius flaps, they follow about the same $\mathrm{C}_{\mathrm{f}}$ along the flap until they encounter a pronounced drop-off and recovery at about an $\mathrm{x} / \mathrm{c}^{\prime}$ of 0.89 . After this "dip" in $\mathrm{C}_{\mathrm{f}}$ the dual radius configurations' skin friction rises back up slightly, where the corresponding length prescribed radius flaps remain at this decreased skin friction slope for the shorter flaps and only slightly rebound for the longer prescribed radius flaps. This gives more insight into why the dual radius configurations experienced 
more drag than the corresponding prescribed radius flaps, but might also be a favorable characteristic for the prescribed radius flaps in keeping the slot flow attached to the flap surface during extreme deflections or lower values of $\mathrm{C}_{\mu}$. This $\mathrm{C}_{\mathrm{f}}$ "dip" is explored in greater depth below in Figure 6-18.

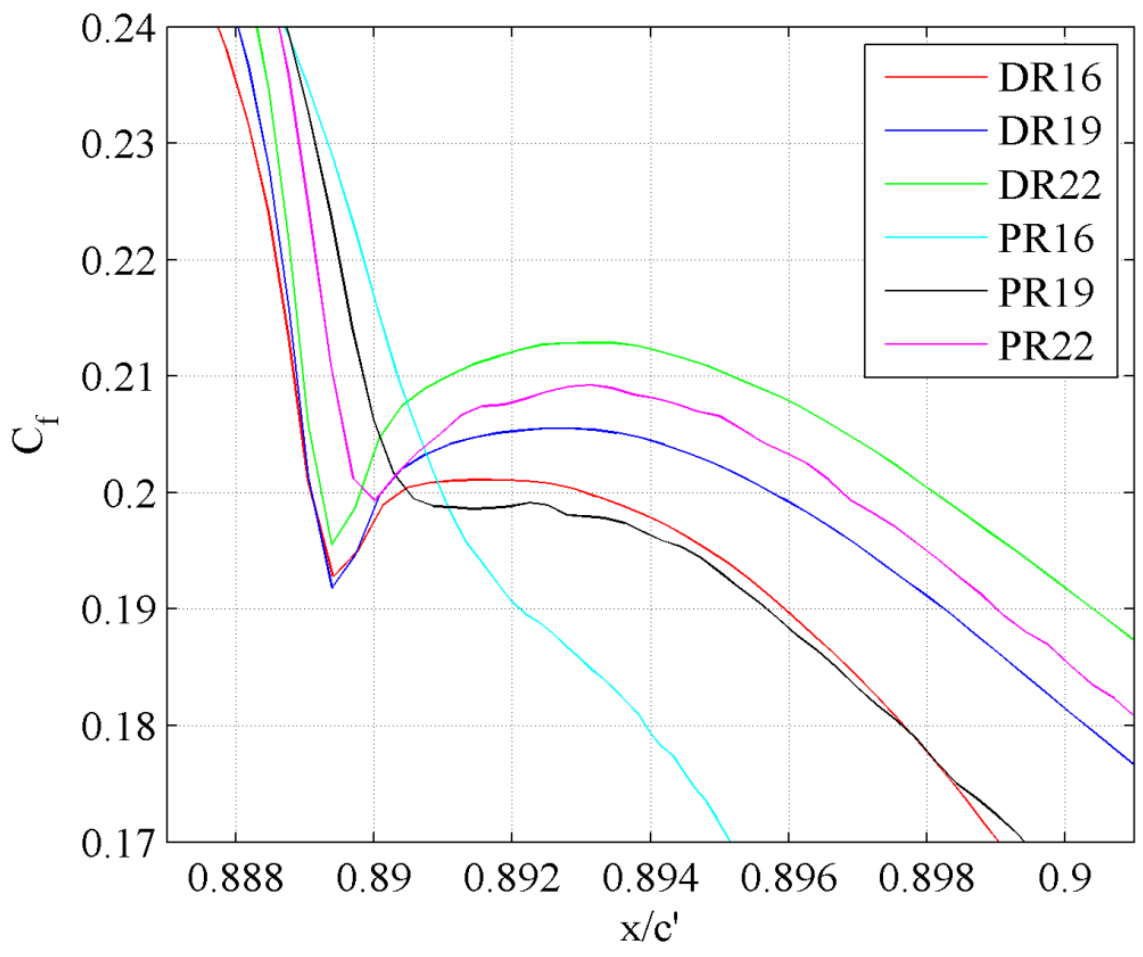

Figure 6-18: Detailed skin friction at flap surface transition, $\alpha=0^{\circ}, 60^{\circ}$ flaps

By examination of the above plot and the geometry of the different flap configurations, it can be noted that this "dip" signifies a large decrease in speed and takes place at the location where each flap transitions from $r_{1}$ into either $r_{2}$ for the dual radius or into the cubically varying radius of the prescribed radius flap. The effect of this curvature change on the skin friction can be seen by the severity of the "dip" encountered by each flap. When tracking the influence of the flap length on the skin friction it appears that the longer the flap, the greater the rebound in $\mathrm{C}_{\mathrm{f}}$ values after the "dip". Comparing the prescribed radius flaps to the dual radius flaps, it is evident that the 
prescribed variation in radius greatly smoothed the sharp fluctuation in skin friction, with PR16 maintaining the smoothest change in the skin friction slope and PR22 having the sharpest change. The severity in skin friction fluctuation was a direct function of how quickly each of the flap configurations' curvature departed from the curvature of the $\mathrm{r}_{1}$ arc on the flap. To visualize this concept a plot of surface curvature for all the flap configurations at this same location is presented below in Figure 6-19. Note that this plot refers to each flap configuration at $60^{\circ}$ deflection, where the change of radius location occurs at the same $\mathrm{x} / \mathrm{c}^{\prime}$-value as the $\mathrm{C}_{\mathrm{p}}$ and $\mathrm{C}_{\mathrm{f}}$ plots of approximately 0.8894 .

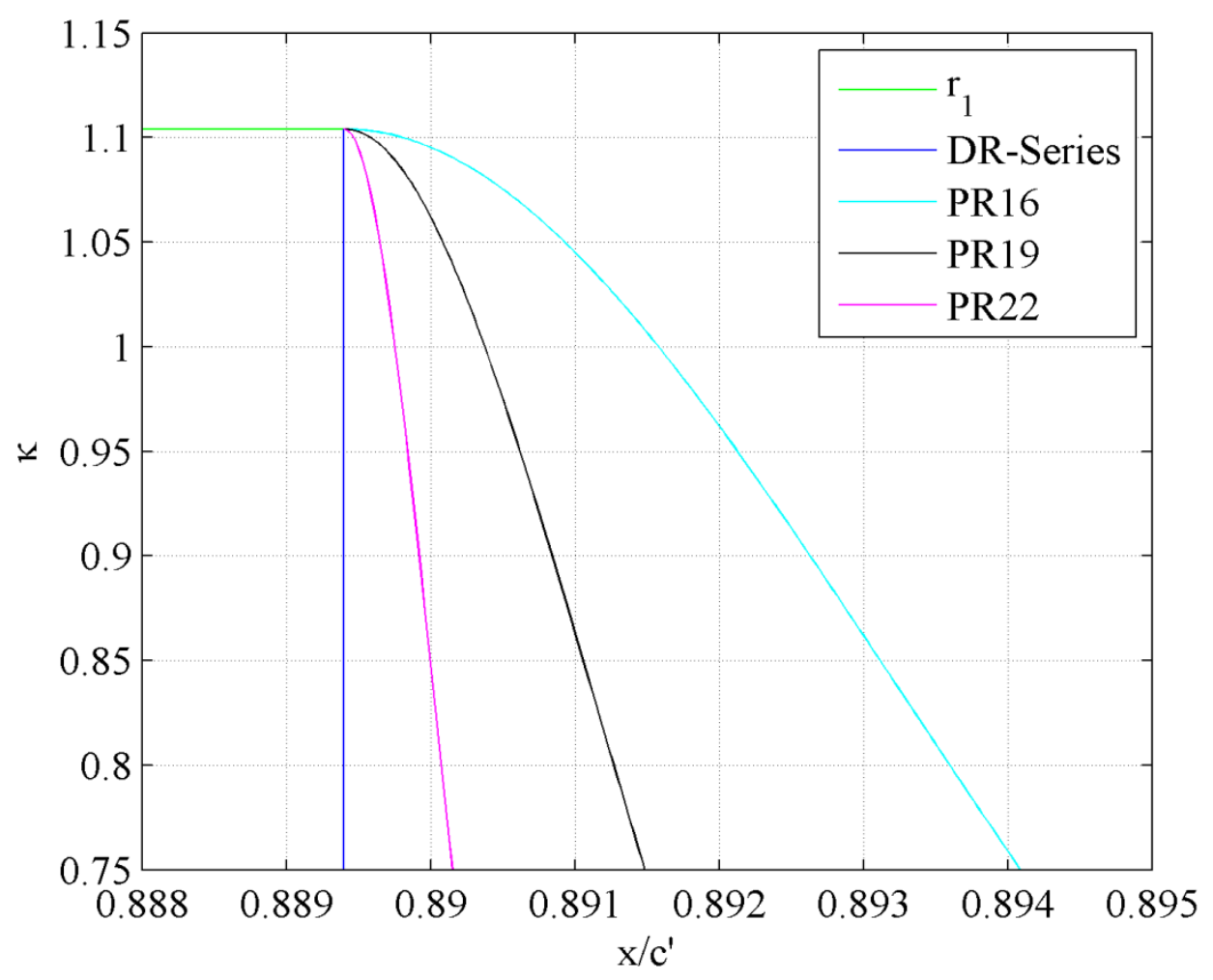

Figure 6-19: Flap curvature at transition from $r_{1}$ to $2^{\text {nd }}$ flap surface $\left(60^{\circ}\right)$

When comparing the flap characteristics presented on the above plot to Figure 6-18, the influence of the behavior each flap displays when departing the $r_{1}$ curvature is more clearly observed. The more rapid the flap's curvature would departure from $r_{1}$, the 
more severe the "dip" in $\mathrm{C}_{\mathrm{f}}$ would be. Considering the prescribed radius configurations, PR22 experienced the most significant $\mathrm{C}_{\mathrm{f}}$ fluctuation due to its curvature quickly departing from the original curvature of $r_{1}$. This also shows how the smooth and gradual curvature changes of PR16 are beneficial in keeping a more continuous skin friction distribution over the entire flap surface, which could assist in encouraging flow attachment over the flap surface during large flap deflections or in the case of a lower $\mathrm{C}_{\mu}$. Another point is that the rapid change in $\mathrm{C}_{\mathrm{f}}$ also coincided with the large pressure increase in the $\mathrm{C}_{\mathrm{p}}$ plots, defining a rapid deceleration of flow. This would propose that more rapid changes in curvature directly influence adverse pressure gradients, which could cause separation at lower values of $\mathrm{C}_{\mu}$ or larger flap deflections. This effect of the changing of flap curvature over the length of the flap surface provides another parameter of $\mathrm{CC}$ geometry that has direct influence on flap performance at a given condition.

The $\mathrm{C}_{\mathrm{f}}$ plots for all the flap configurations at $\alpha=-5^{\circ}$ and $\alpha=3^{\circ}$ stayed consistent with the $\alpha=0^{\circ}$ plot, and have been omitted from the results section. Note that these plots are located in the appendix for reference.

Figure 6-20 below presents the wall $\mathrm{y}^{+}$values encountered during the simulation of the $60^{\circ}$ deflection at $\alpha=0^{\circ}$. 


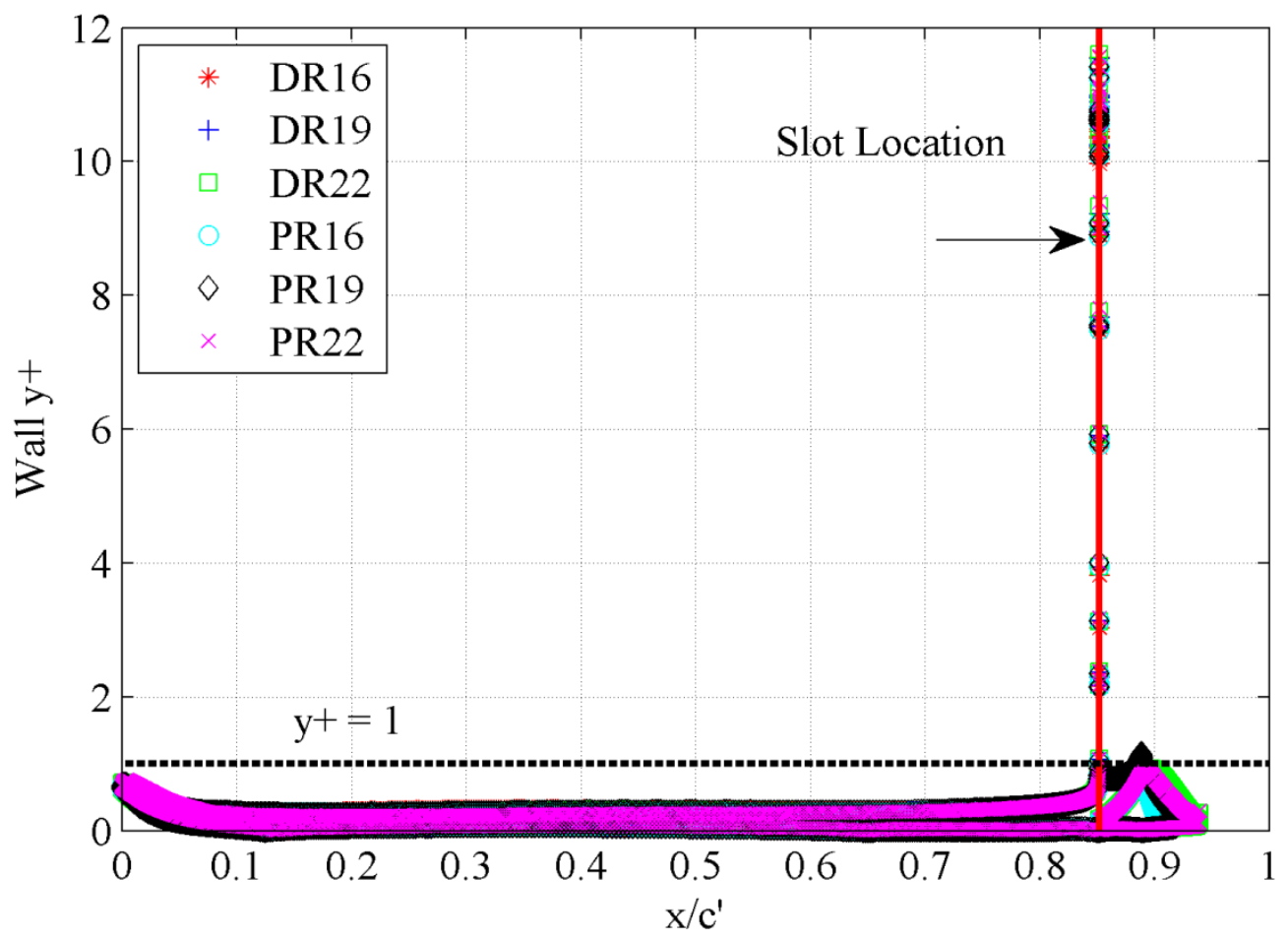

Figure 6-20: $y^{+}$values of the airfoil/flap configurations, $\alpha=0^{\circ}, 60^{\circ}$ flaps

The $\mathrm{y}^{+}$values for all of the different flap configurations stayed at one or below, except for the slot location. The same problem was encountered by Lee-Rausch, ${ }^{52}$ who reduced spacing by a factor of four to see little difference in solution. Reduction of the cell height at the slot would significantly drive up cell count, greatly increasing solving time, and not significantly change the results. For these reasons, the resulting $\mathrm{y}^{+}$values were deemed adequate. The $\mathrm{y}^{+}$values for $\alpha=-5^{\circ}$ and $\alpha=3^{\circ}$ stayed consistent with those of the $\alpha=0^{\circ}$ case, thus not displayed. These plots are presented within the appendix for reference.

\section{Flap Parameter Trends}

To quantify the influence of each of the flap parameters on takeoff performance, trends for both dual radius and prescribed radius flaps were developed. With high lift, 
low drag, and low negative pitching moment desired for takeoff operations, the performance parameters of interest for the $60^{\circ}$ flap deflection were $L / D$ and $C_{m},(c / 4)$. The geometric parameters that were investigated on each flap shape (dual radius/prescribed radius) were the flap's departure angle $(\theta)$, the flap chord-to-airfoil chord ratio $\left(\mathrm{c}_{\mathrm{f}} / \mathrm{c}^{\prime}\right)$, and the radius ratio $\left(r_{2} / r_{1}\right.$ or $\left.r_{2 \_a v g} / r_{1}\right)$. Although each geometric parameter is dependent upon the others, each parameter was analyzed separately in attempt to determine each parameter's influence on the separate takeoff performance parameters studied. Note that all flap performance was analyzed at $\alpha=0^{\circ}$.

Figure 6-21 and Figure 6-22 below display the takeoff performance trends of the flap's departure angle $(\theta)$.

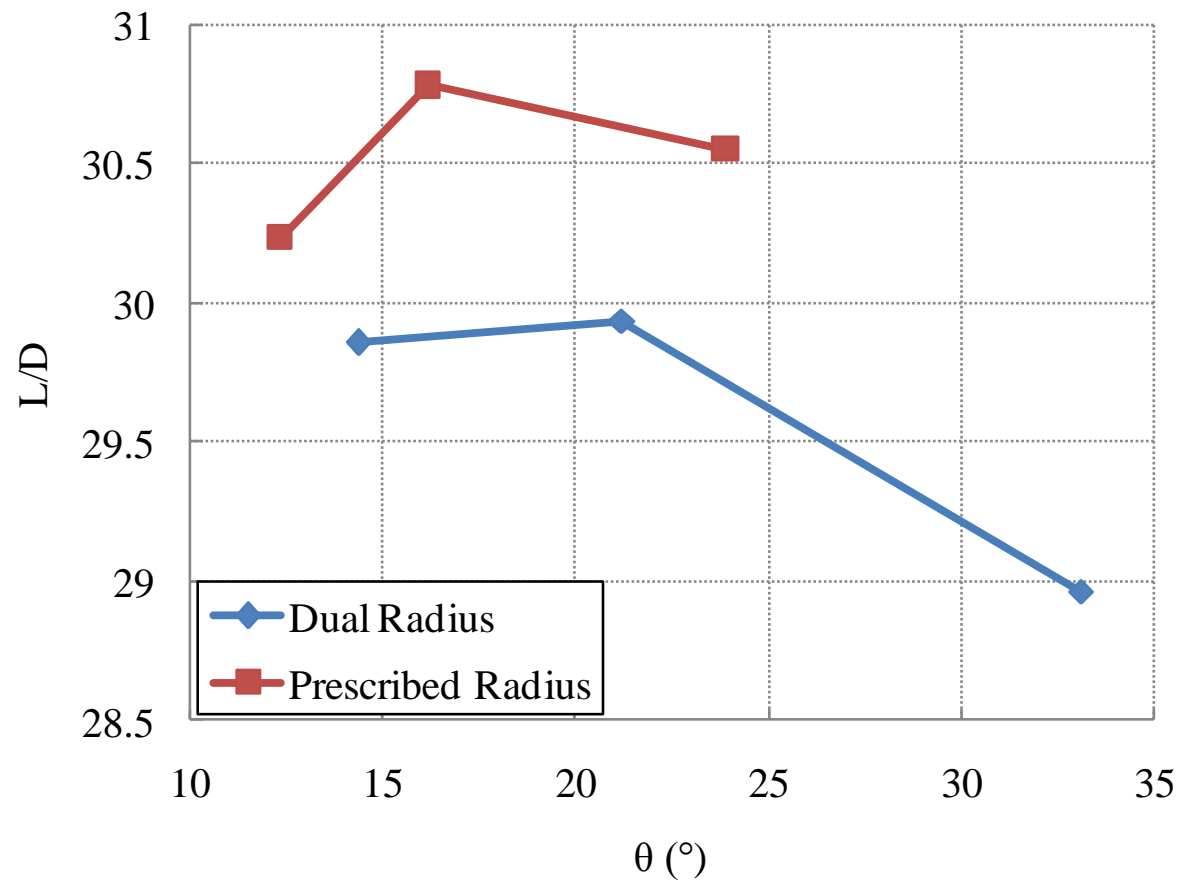

Figure 6-21: Lift-to-drag ratios for flap configurations given $\theta$ 


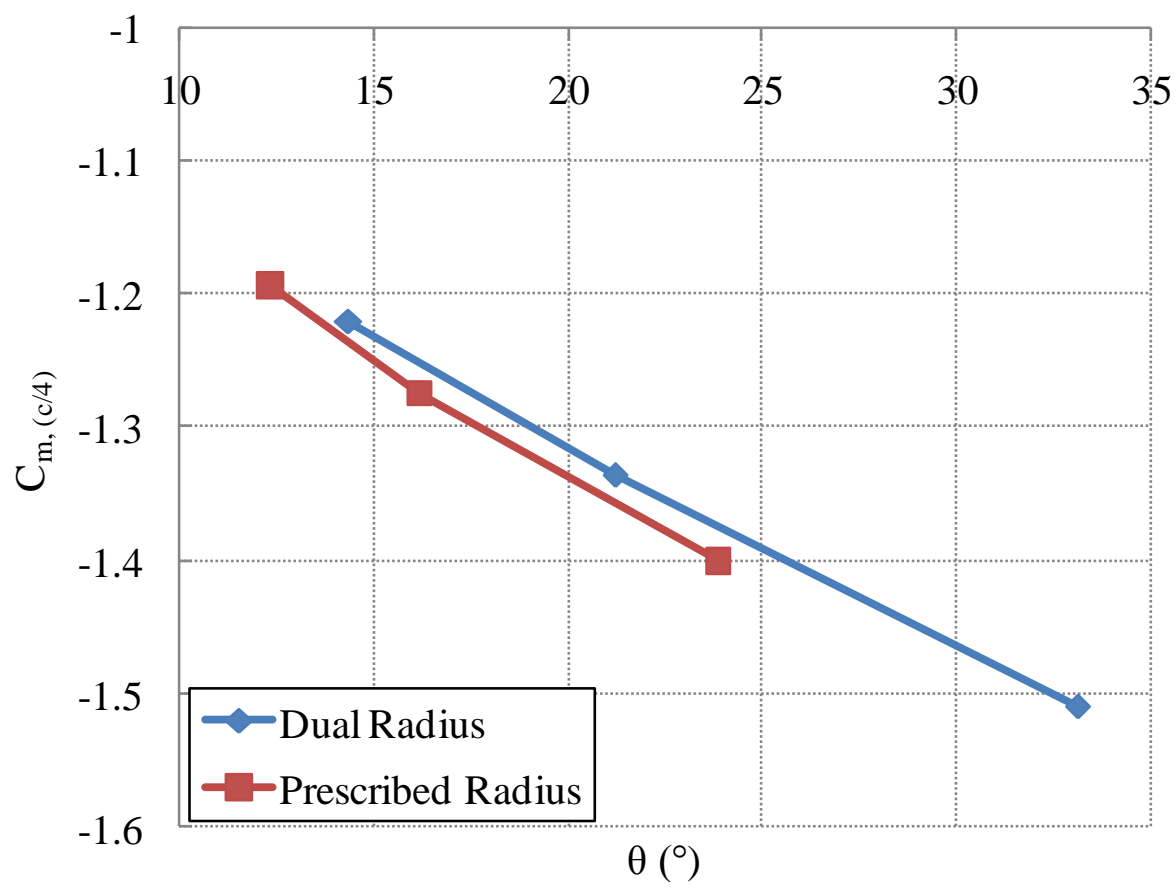

Figure 6-22: Pitching moment for flap configurations given $\theta$

The L/D plot above displays how both flap configurations experience the global maximum L/D between the $\theta$-values investigated. Maximizing the L/D performance parameter was of the most interest for the takeoff setting, although the $\mathrm{C}_{\mathrm{m} \text {, (c/4) }}$ parameter was also important to track in the complete design of an aircraft to appropriately size tail surfaces to counter the large nose-down pitching moments caused by the CC. It is also interesting to note that the $\mathrm{C}_{\mathrm{m} \text {, (c/4) }}$ displayed a close to linear trend with $\theta$.

With the current flap configurations, the approximate maximum L/D occurs at a $\theta$ of $16^{\circ}$ for the prescribed radius with an L/D of 30.8 and $\theta$ of $21^{\circ}$ for the dual radius with an L/D of about 30. Although both flap configurations have very similar L/D values, the prescribed radius flap achieves a slightly higher $\mathrm{L} / \mathrm{D}$ at a lower $\theta$. When taking the approximate $\theta$ at which the maximum L/D occurs for each configuration, the resulting

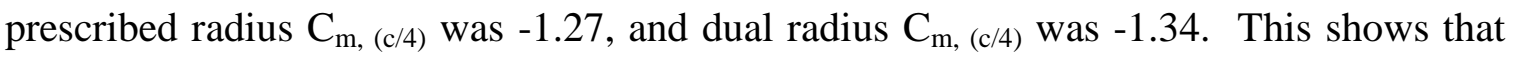
even though the prescribed radius reaches a larger L/D, it still produced a very similar 
negative $C_{m}$, (c/4) magnitude. It should be noted that by observing the above charts, the prescribed radius flap configuration can achieve a higher L/D than the dual radius at larger $\theta$ values than its local maximum.

Figure 6-23 and Figure 6-24 below depict the same takeoff performance trends given the flap chord-to-modified airfoil chord length ratio $\left(\mathrm{c}_{\mathrm{f}} / \mathrm{c}^{\prime}\right)$.

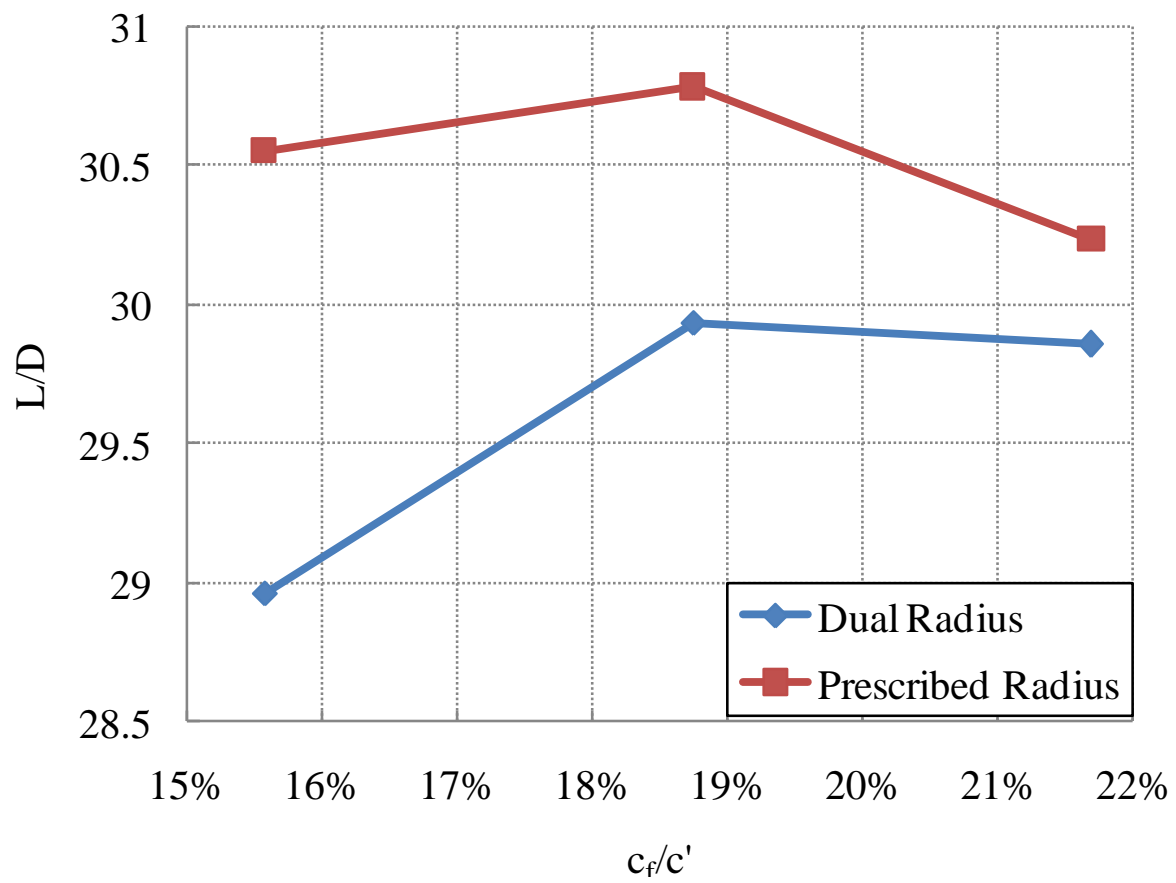

Figure 6-23: Lift-to-drag ratios for flap configurations given $c_{\mathrm{f}} / \mathrm{c}^{\prime}$ 


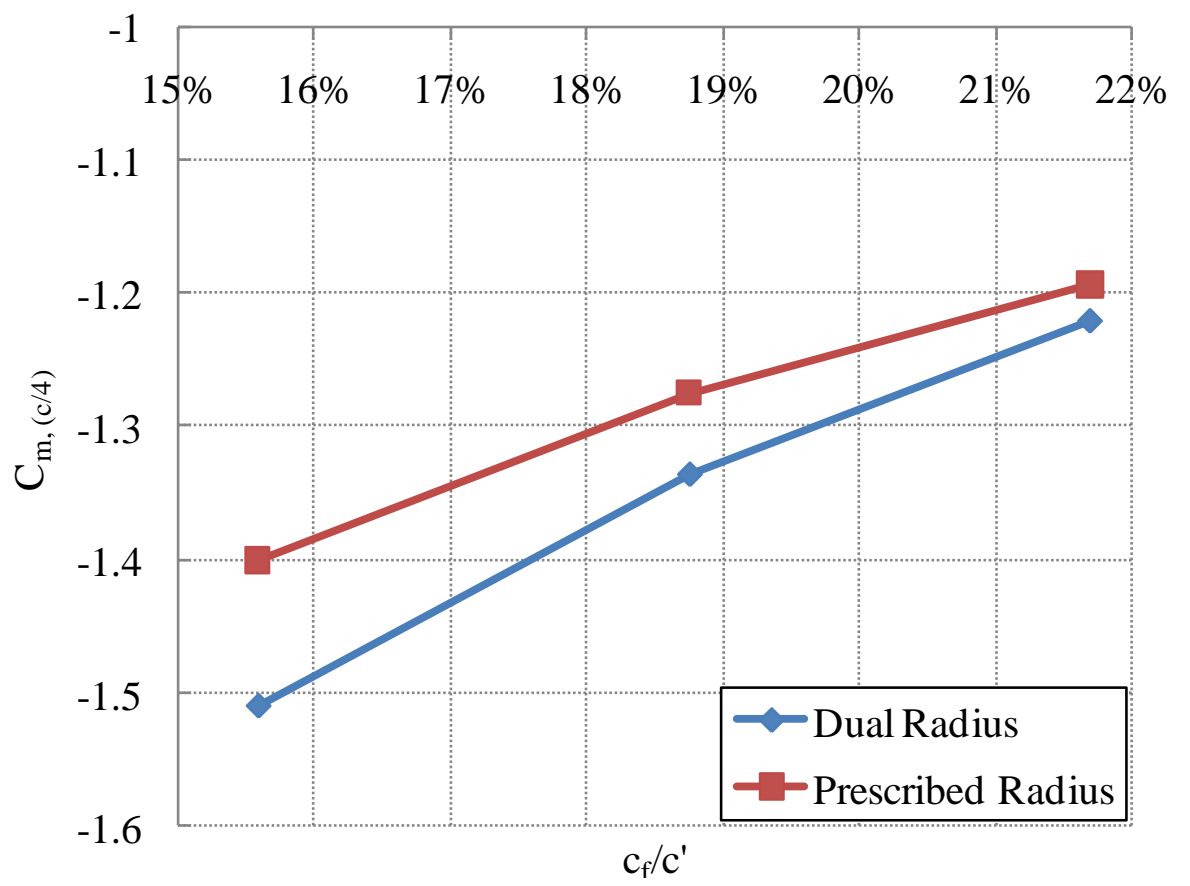

Figure 6-24: Pitching moment for flap configurations given $\mathrm{c}_{\mathrm{f}} / \mathrm{c}^{\prime}$

The above trends suggest the prescribed radius flap reaches its maximum L/D at a $\mathrm{c}_{\mathrm{f}} / \mathrm{c}^{\prime}$ slightly less than $19 \%$, where the dual radius configuration achieves its maximum L/D at a slightly longer flap length than $19 \%$. Similar to the takeoff performance trends with $\theta$, the resulting $\mathrm{C}_{\mathrm{m} \text {, (c/4) }}$ of the shorter prescribed radius is less (or more negative) than those of the dual radius maximum $\mathrm{L} / \mathrm{D} \mathrm{c}_{\mathrm{f}} / \mathrm{c}^{\prime}$. Again, it should be noted that the prescribed radius flap configuration can achieve a larger L/D along with less negative $C_{m}$, (c/4) magnitude than the dual radius flap with a larger $\mathrm{c}_{\mathrm{f}} / \mathrm{c}^{\prime}$ than its local maximum.

Figure 6-25 and Figure 6-26 below display the takeoff performance trends for the radius ratio $\left(\mathrm{r}_{2} / \mathrm{r}_{1}\right)$ of the dual radius flaps. The prescribed radius ratio $\left(\mathrm{r}_{2 \_ \text {avg }} / \mathrm{r}_{1}\right)$ performance trends are shown in Figure 6-27 and Figure 6-28. Note that the dual radius and prescribed radius configurations were plotted separately due to the difference in $\mathrm{r}_{2} / \mathrm{r}_{1}$ to $r_{2 \_a v g} / r_{1}$ magnitudes. 


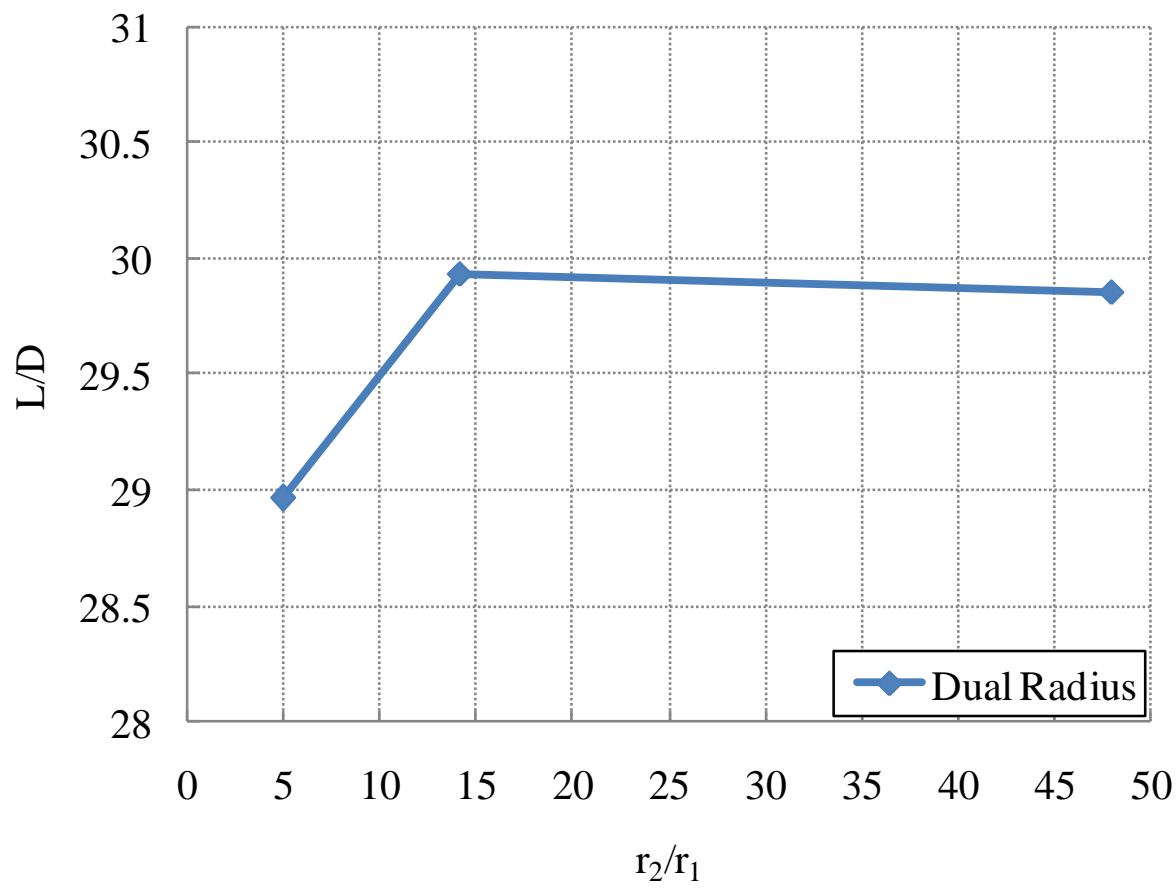

Figure 6-25: Lift-to-drag ratios for dual radius given $\mathbf{r}_{2} / \mathbf{r}_{1}$

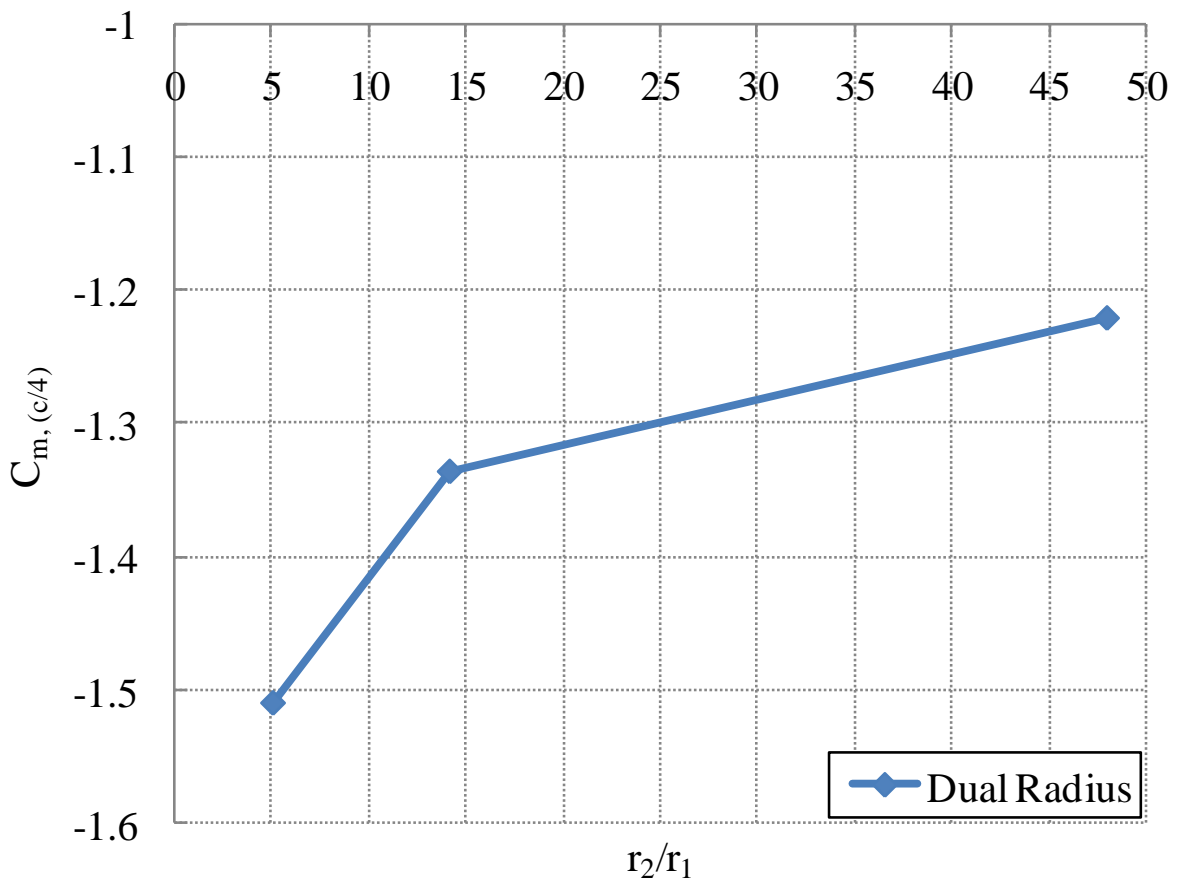

Figure 6-26: Pitching moment for dual radius given $r_{2} / \mathbf{r}_{1}$ 


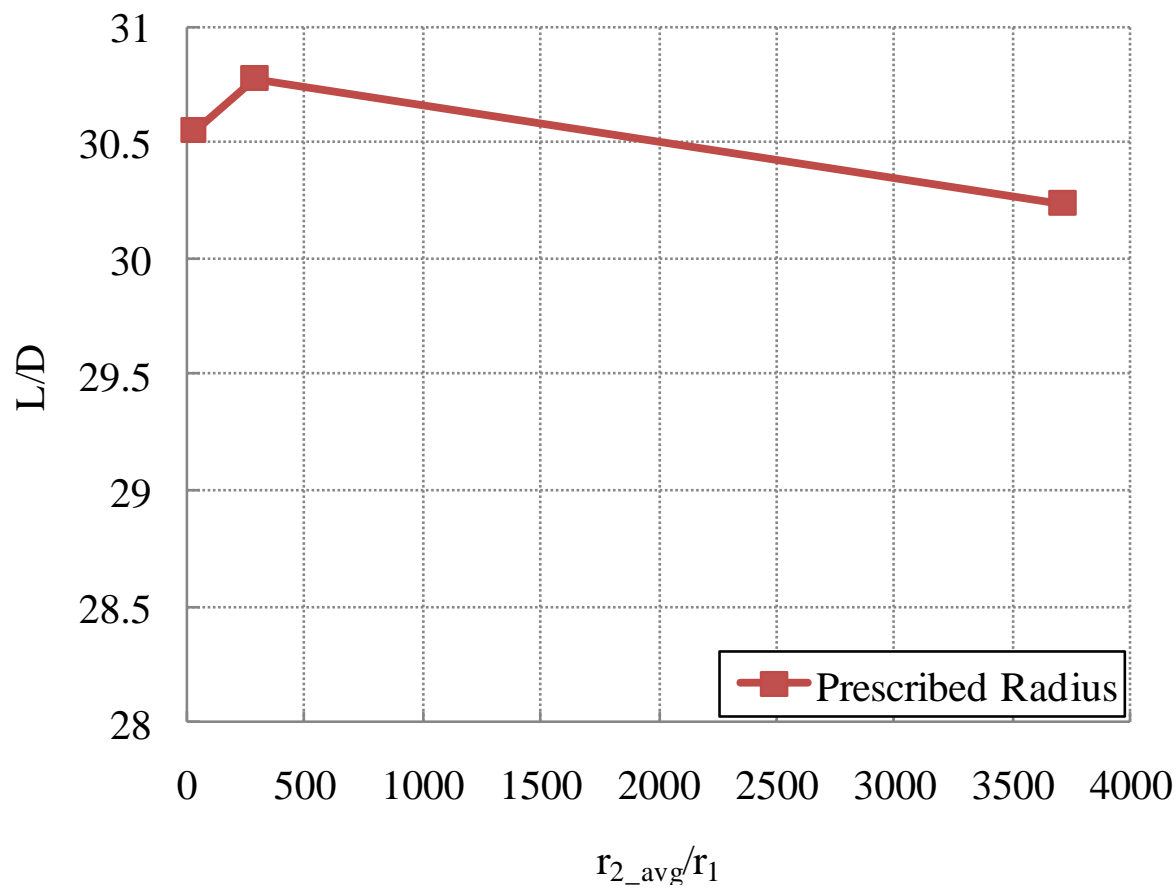

Figure 6-27: Lift-to-drag ratios for prescribed radius given $\mathbf{r}_{2 \_ \text {avg }} / \mathbf{r}_{1}$

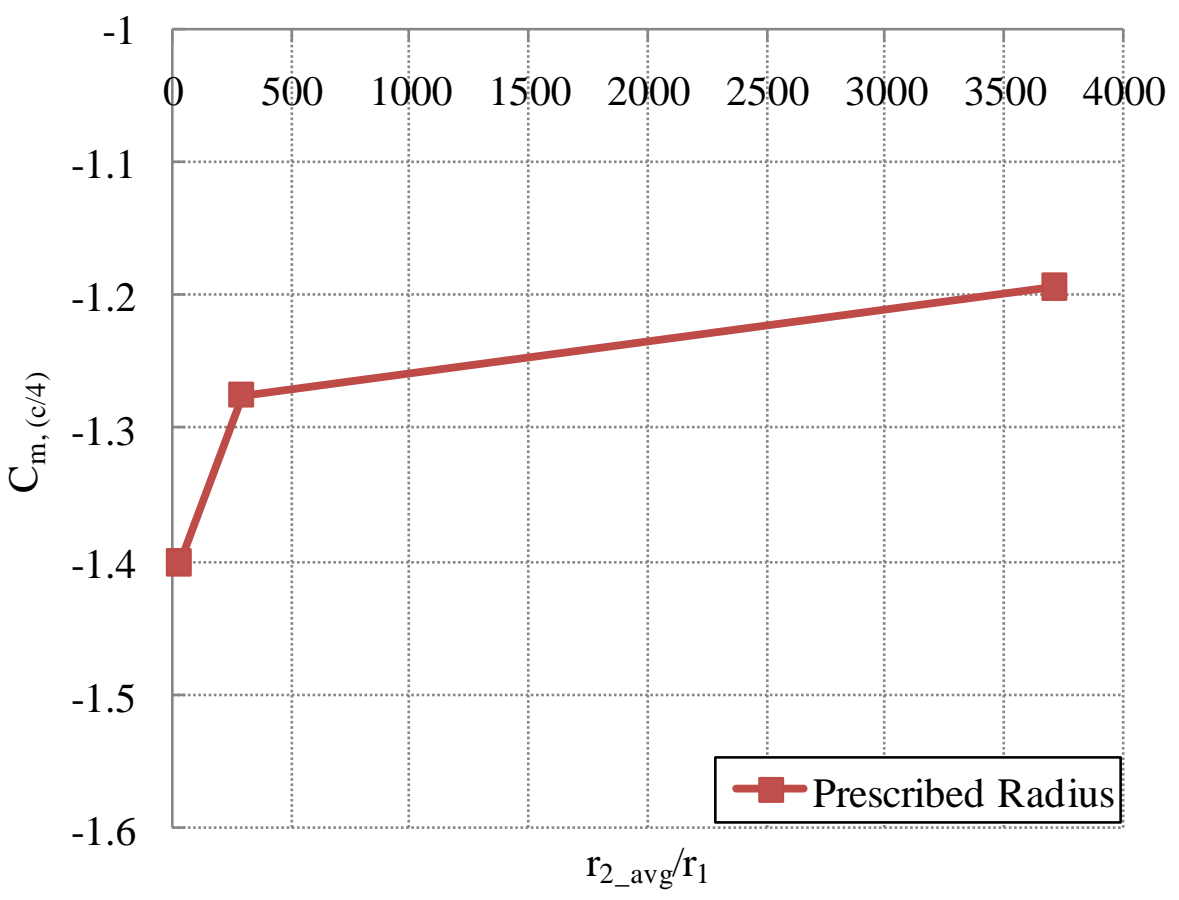

Figure 6-28: Pitching moment for prescribed radius given $\mathbf{r}_{2 \_a v g} / \mathbf{r}_{1}$ 
Both dual radius and prescribed radius configurations experienced similar trends with each of the takeoff performance parameters. The prescribed radius configuration again reaches larger $\mathrm{L} / \mathrm{D}$ values, although the trend of the $\mathrm{L} / \mathrm{D}$ behavior is skewed due to the close proximity of two of the $r_{2 \_a v g} / r_{1}$ values on the $x$-axis compared to the third data point. The trends result in the dual radius flaps reaching a maximum L/D at a relatively larger radius ratio and the prescribed radius flaps reaching its maximum $\mathrm{L} / \mathrm{D}$ value at relatively lower radius ratios, staying consistent with the previous performance trends. Unlike the performance trends of $\theta$ and $c_{f} / c^{\prime}$, the trends of the radius ratios suggest the prescribed radius configuration achieves a less negative $C_{m}$, (c/4) value than dual radius configuration at their estimated maximum L/D's. Again it should be noted that the prescribed radius flap trends span a large $\mathrm{r}_{2 \_ \text {avg }} / \mathrm{r}_{1}$ gap between the second and third data points. To obtain more meaningful performance trends with the radius ratio flap parameter, it is recommended that additional data points be solved within the spaces between the presented radius ratio data for more refined performance trends.

\section{Approach/Landing Performance - Low Airspeed, $90^{\circ}$ Flaps}

The resulting $90^{\circ}$ flap Mach contours and streamlines for the approach/landing conditions are shown below in Table 6-2. For $\alpha=-5^{\circ}$ and $\alpha=3^{\circ}$ Mach contour and streamline results, refer to the appendix. 
Table 6-2: $90^{\circ}$ flap streamlines and contours of Mach at $\alpha=0^{\circ}$

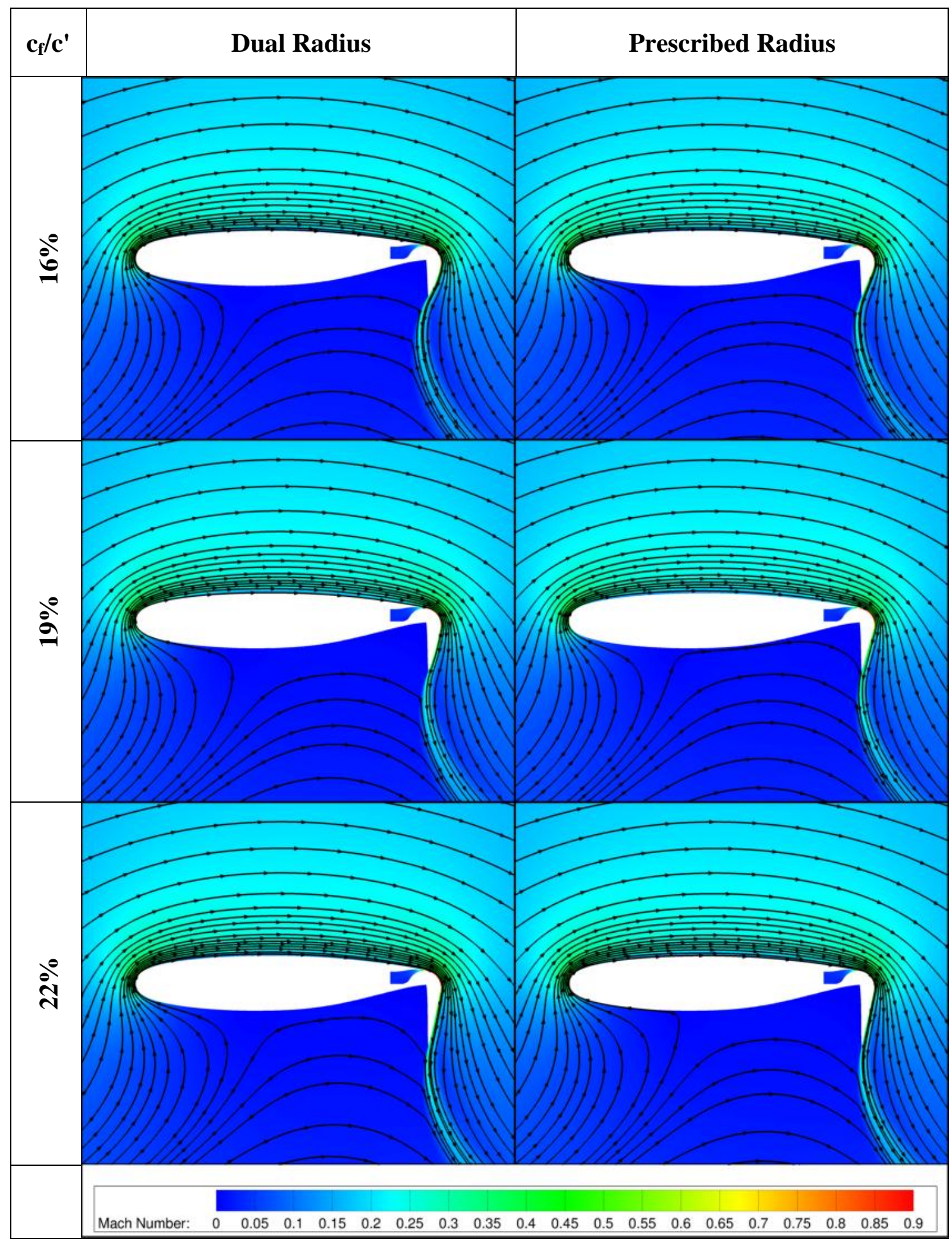


All approach/landing simulations did not experience any sonic flow within any of the computational domains. The first observation that can be made from the above table of the $90^{\circ}$ flap configurations is the extreme aft location of the stagnation point for all of the flaps. When comparing these contours and streamlines to those of the $60^{\circ}$ deflection, the significant streamline curvature and increased velocity in the regions of the leading edge and slot location are notable. The flap configurations do not hold consistent trends with length in flap length or flap shape (dual radius vs. prescribed radius). For the dual radius configurations, as the flap length increased from DR16 to DR 19, the stagnation point initially moved aft which opposes the trend seen for the $60^{\circ}$ deflection. Though as the length increase from DR19 to DR22, the stagnation location moves forward. Comparing the dual radius to the prescribed radius configurations of each length, the dual

radius flaps have further aft stagnation locations, with the exception of the $22 \%$ flap lengths. This suggests that the dual radius configurations are still augmenting greater forces than the prescribed radius.

\section{Lift, Drag, and Moment Data}

To further investigate the variation in stagnation location movement the analytical plots of $C_{1}$ vs. $\alpha$ and $C_{d}$ vs. $\alpha$ were generated and are presented in Figure 6-29 and Figure 6-30, respectively. 


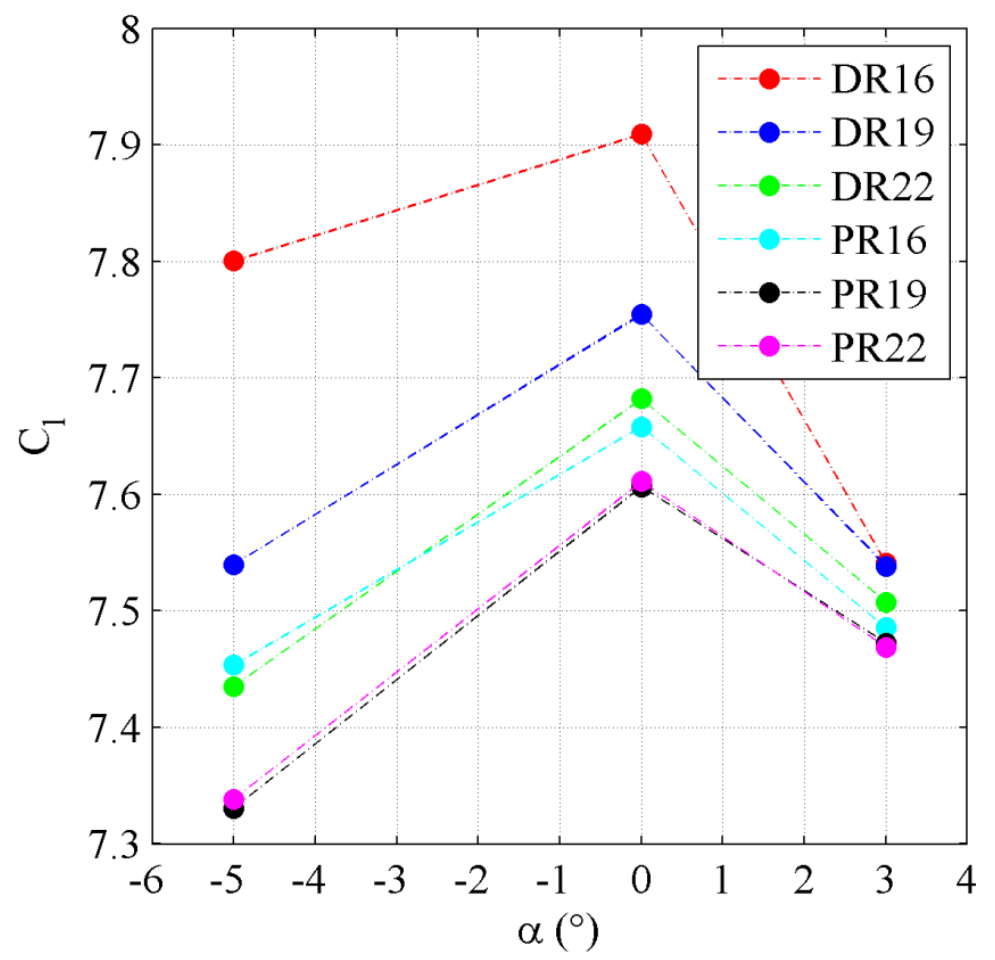

Figure 6-29: Lift for $90^{\circ}$ flap configurations

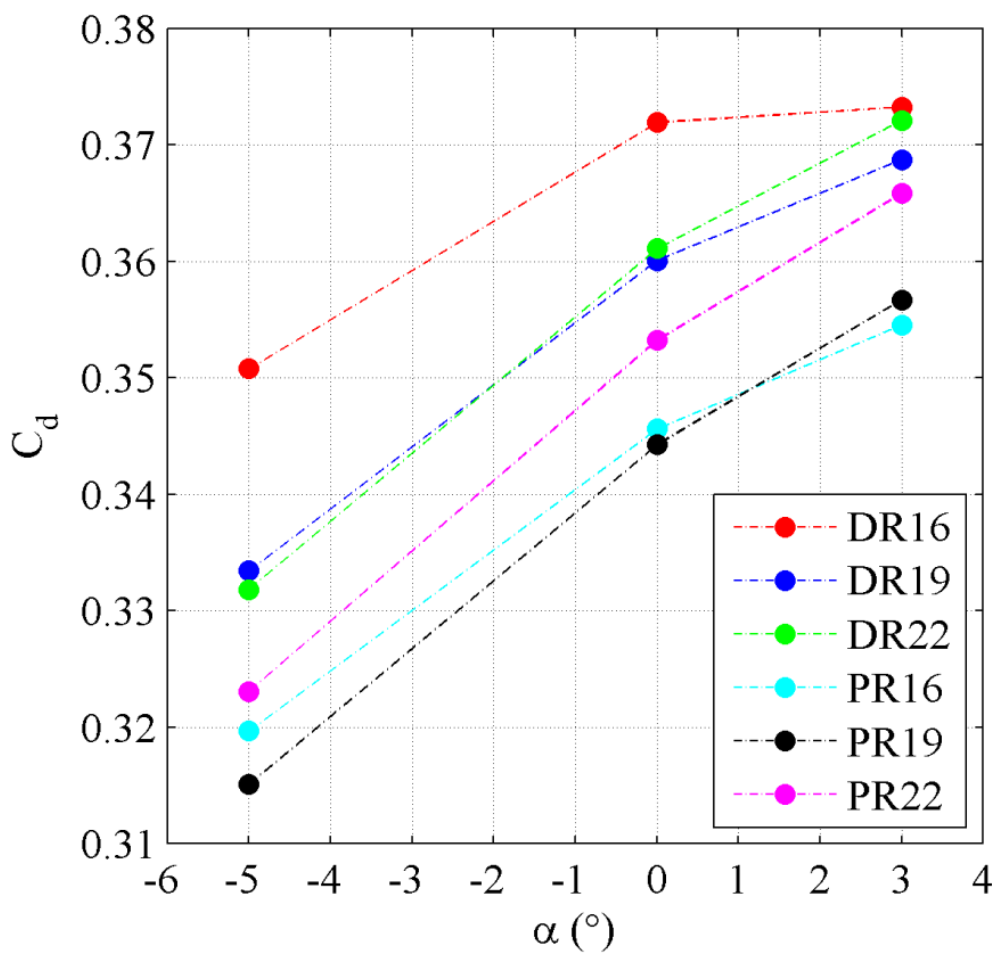

Figure 6-30: Drag for $90^{\circ}$ flap configurations 
Examining the lift curves of the different flap configurations above shows how every one of the flaps achieved their maximum lift at $\alpha=0^{\circ}$, and as $\alpha$ increases they began or continue to lose lift. The difference in lift values between the minimum and maximum performing flap configurations significantly dropped from $6 \%$ at $\alpha=-5^{\circ}$ to $1 \%$ at $\alpha=3^{\circ}$, showing that all configurations produced a similar lift at this condition. DR16 attained the largest average $\mathrm{C}_{1}$, with a maximum value of 7.9 at $\alpha=0^{\circ}$. Although the drop-off in DR16's lifting performance was most severe with a -4.7\% drop from an $\alpha$ of $0^{\circ}$ to $3^{\circ}$, showing that this configuration experienced the most unfavorable behavior when pursuing approach/landing performance. The dual radius configurations all attained higher lift than the respective length prescribed radius flap. Both PR19 and PR22 generate very similar lift curves, which insinuate PR22's lifting performance is not as adversely affected by the extreme flap deflections or relatively high $\alpha$ values (when considering the amount of flow turning). Observing the slopes of the lift curves between $\alpha=0^{\circ}$ and $\alpha=3^{\circ}$ show the prescribed radius flaps demonstrated improved flap characteristics over the dual radius flaps, maintaining greater lift curve slopes as the corresponding length dual radius flaps.

From Figure 6-30, all the different flap configurations experience a leveling off of drag rise as $\alpha$ was increased. This can be accredited to each of the flaps reaching their respective maximum $\mathrm{C}_{\mathrm{l}}$, with the flap that endured the largest lift drop also having the most significant reduction in drag slope (DR16). For the dual radius flaps the best lifting DR16 configuration incurred the largest drag values and the DR19 and DR22 flaps displaying similar drag. The prescribed radius flaps showed a different behavior with 
PR22 having the most drag and PR16 and PR19 had comparable values although PR16 produced the greater lift.

Plots of $C_{d}$ vs. $C_{1}$ and L/D vs. $\alpha$ are displayed below in Figure 6-31 and Figure 6-32 to examine the direct lift-to-drag relations of each flap configuration.

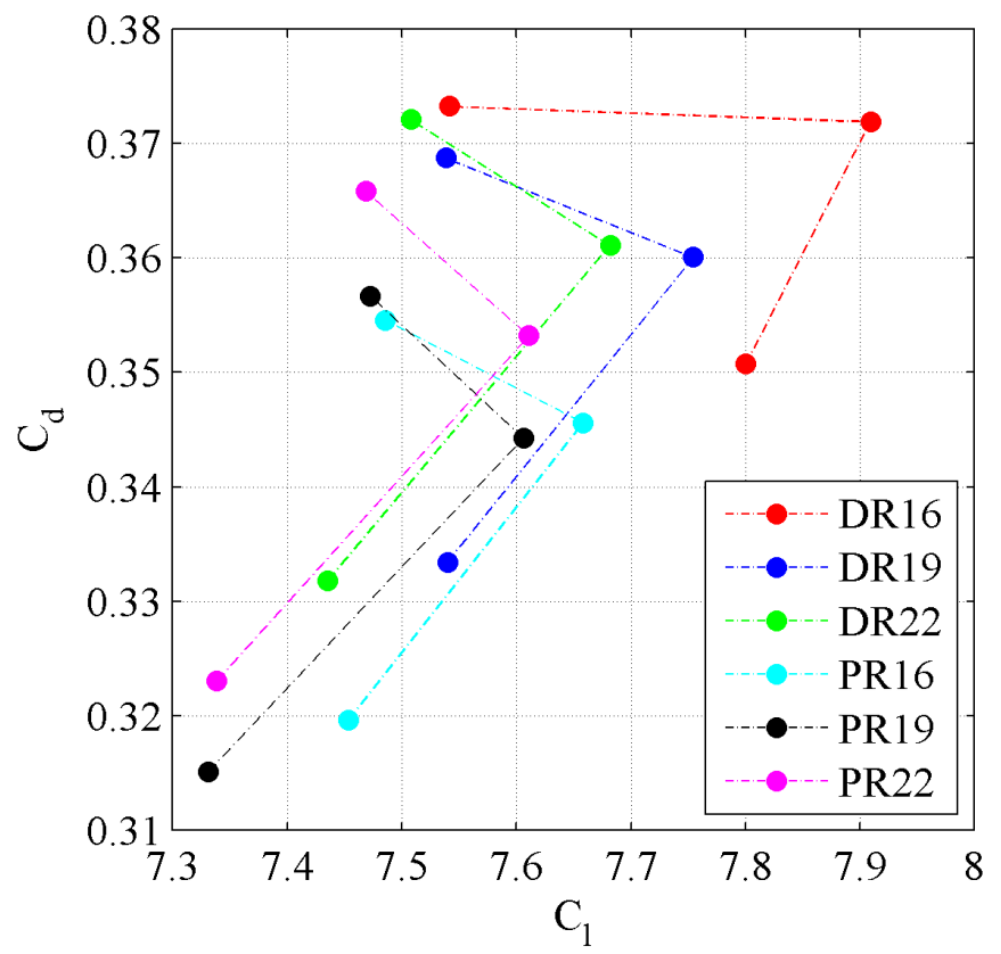

Figure 6-31: Drag given lift for $90^{\circ}$ flap configurations 


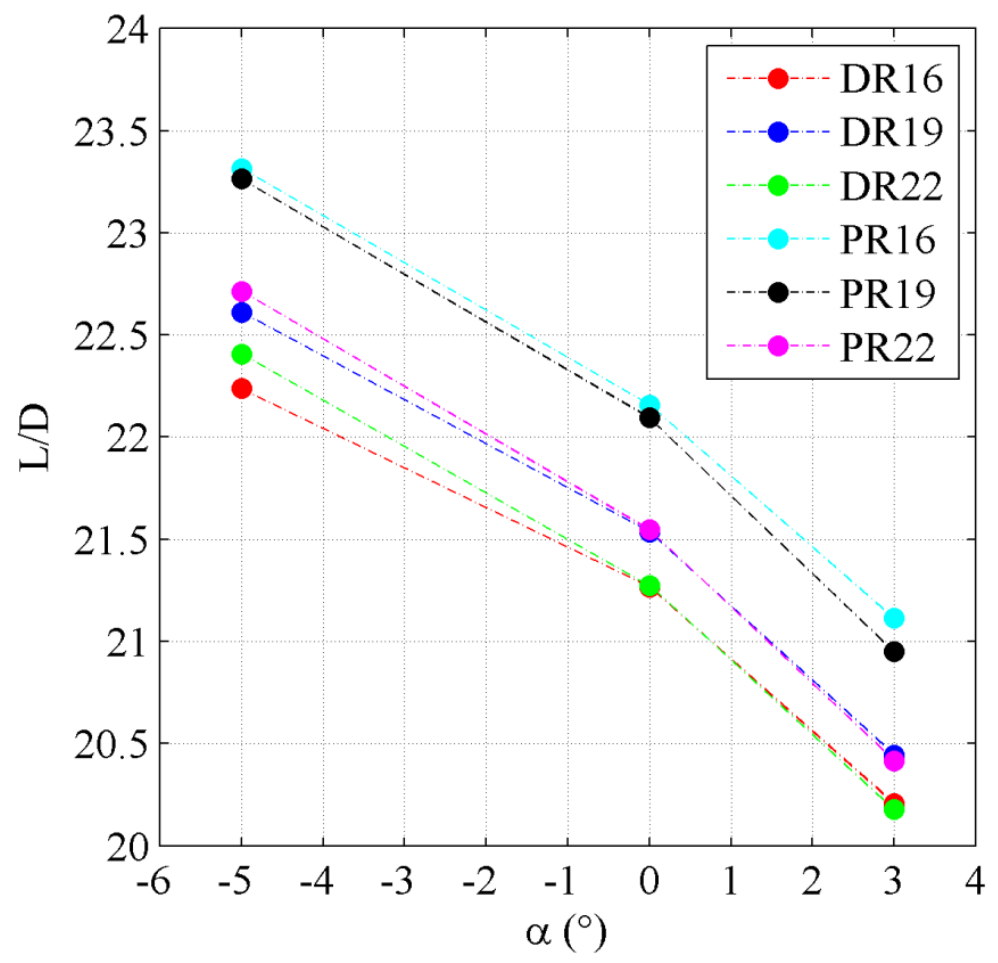

Figure 6-32: Lift-to-drag ratios for $90^{\circ}$ flap configurations

From Figure 6-31, the flap configurations demonstrate much more sporadic behavior in lift and drag when compared to the $C_{d}$ vs. $C_{1}$ plot of the $60^{\circ}$ flap deflection (Figure 6-3). The lift and drag augmentation benefits of DR16 can be more clearly seen over the rest of the configurations, although the severity of its performance loss due to $\alpha$ is also evident. The most desirable traits of airfoil performance during approach/landing are high lift and high drag, which would target the flap configuration that reaches the furthest into the upper-right region of the $C_{d}$ vs. $C_{1}$ plot which would be DR16. Although the severe lift and drag loss experienced during high $\alpha$ 's of the DR16 flap, makes this configuration less desirable. Considering both flap characteristics directs the selection of the "best" performing approach/landing configuration towards a flap that maintains high lift and drag values but also has docile lift and drag characteristics at high $\alpha$ 's, which is accomplished by both configurations of the $22 \%$ flap. 
L/D vs. $\alpha$ of the $90^{\circ}$ flap configurations (Figure 6-32) followed very comparable trends as the $60^{\circ}$ plot (Figure 6-4), although being offset in magnitude. The $60^{\circ}$ flap deflection average $\mathrm{L} / \mathrm{D}$ value for $\alpha=0^{\circ}$ was 30.1 where the $90^{\circ}$ flap averages 21.6, showing a $28 \%$ reduction and how the $90^{\circ}$ deflection generates much more drag per lift produced than the $60^{\circ}$ deflection. These characteristics make this flap deflection much more desirable for approach/landing mission segments.

Additional L/D and the pitching moment data of the approach/landing settings for the six different flap configurations are presented below in Figure 6-33 and Figure 6-34, respectively.

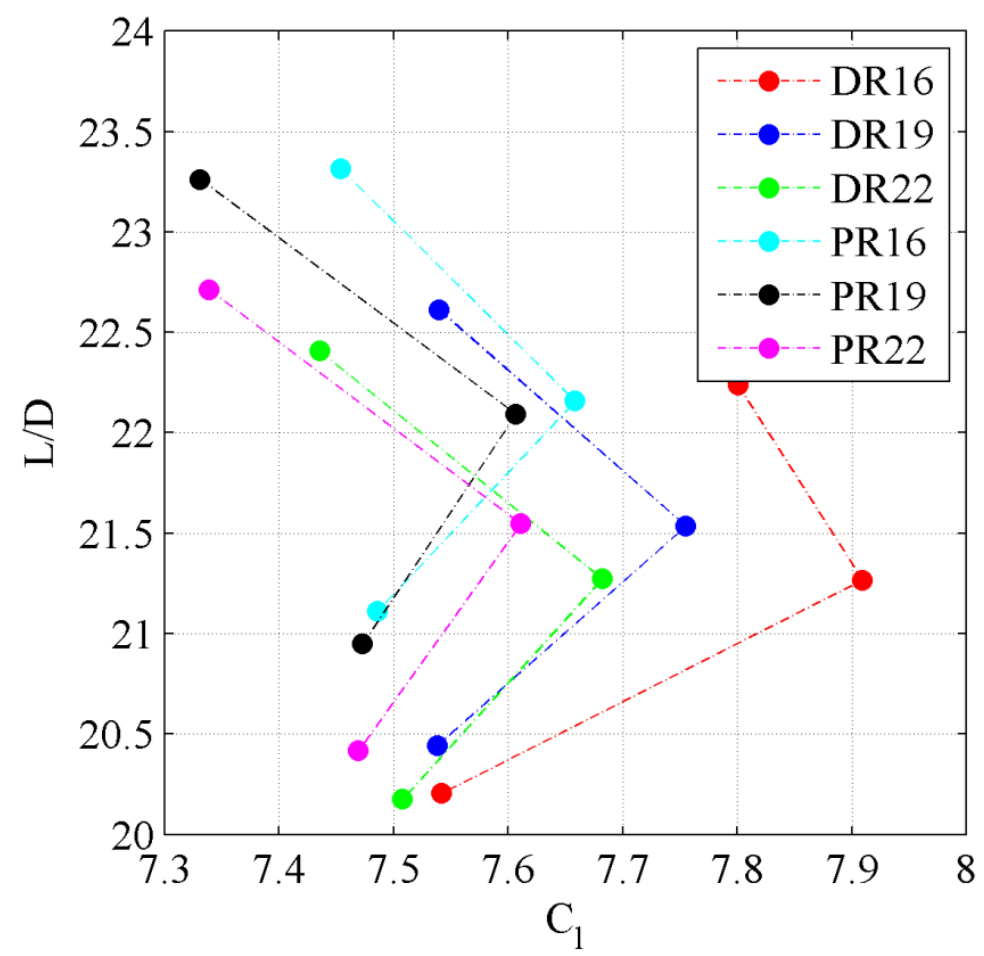

Figure 6-33: Lift-to-drag ratios given lift for $90^{\circ}$ flap configurations 


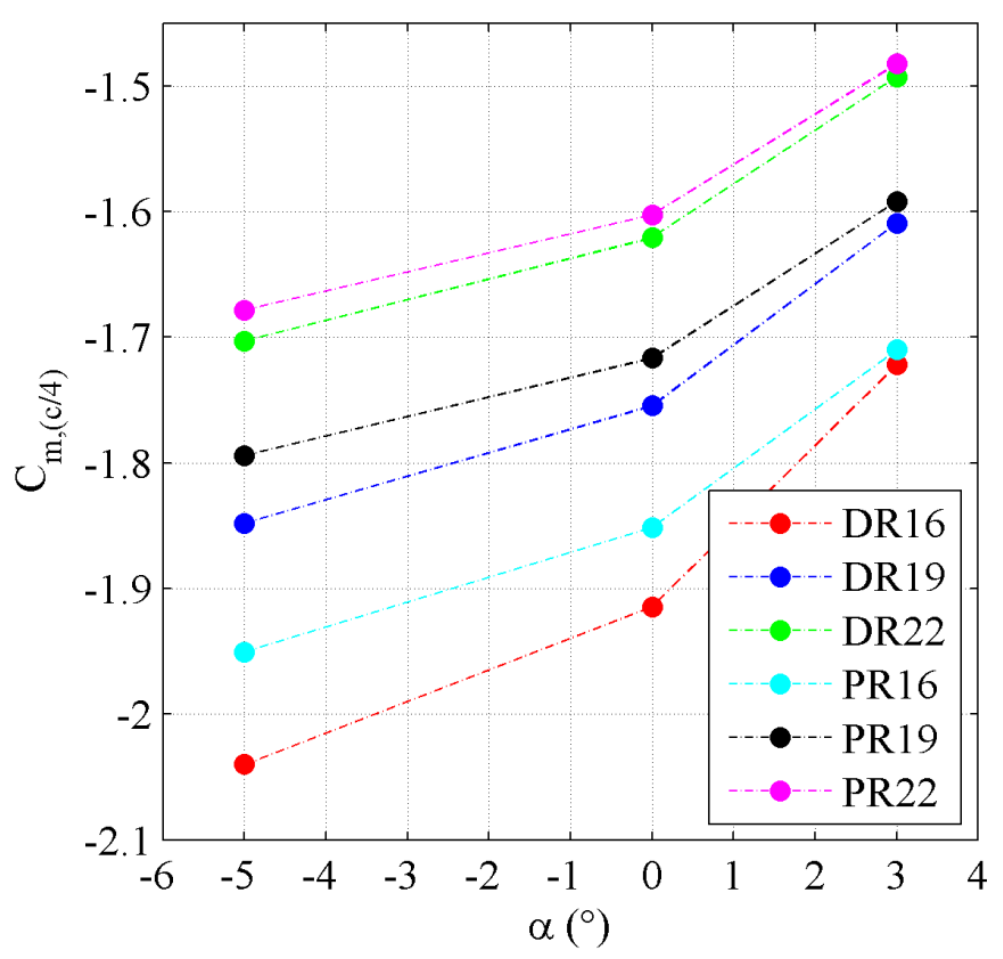

Figure 6-34: Pitching moment for $90^{\circ}$ flap configurations

The L/D ratios initially follow the same trend as the $60^{\circ}$ flap trends (Figure 6-5), but then sharply depart where the flap configurations begin to severely lose lift while still increasing drag, on the lower section of the plot. The prescribed radius configurations again maintain larger L/D ratios given lift generated than their respective length dual radius flap, except for one case with PR22. This case occurred where all flap configurations lost significant lift.

Comparing the $\mathrm{C}_{\mathrm{m},(\mathrm{c} / 4)}$ vs. $\alpha$ data above to the corresponding data of $60^{\circ}$ flap deflection (Figure 6-6) shows the significant increase in negative pitching moment with the larger flap deflection. All of $90^{\circ}$ flaps encountered a significant reduction in negative pitching moment as $\alpha$ increases and the flaps begin to lose lift, where the $60^{\circ}$ flaps demonstrated a more constant moment over all $\alpha$ 's. The length of each flap had the most influence on pitching moment, with longer flaps reducing the negative pitching moment. 
Each of the prescribed radius configurations had a slight reduction in moment over the corresponding length dual radius flap, which can be related to the increased curvature carried throughout the surface of the dual radius flaps.

\section{Pressure and Skin Friction Data}

The $\mathrm{C}_{\mathrm{p}} \mathrm{vs} . \mathrm{x} / \mathrm{c}^{\prime}$ plot for $\alpha=0^{\circ}$ at the approach/landing flap setting of $90^{\circ}$ is shown below in Figure 6-35. Again, the red vertical lines in each of the figures represent the slot location of each airfoil.

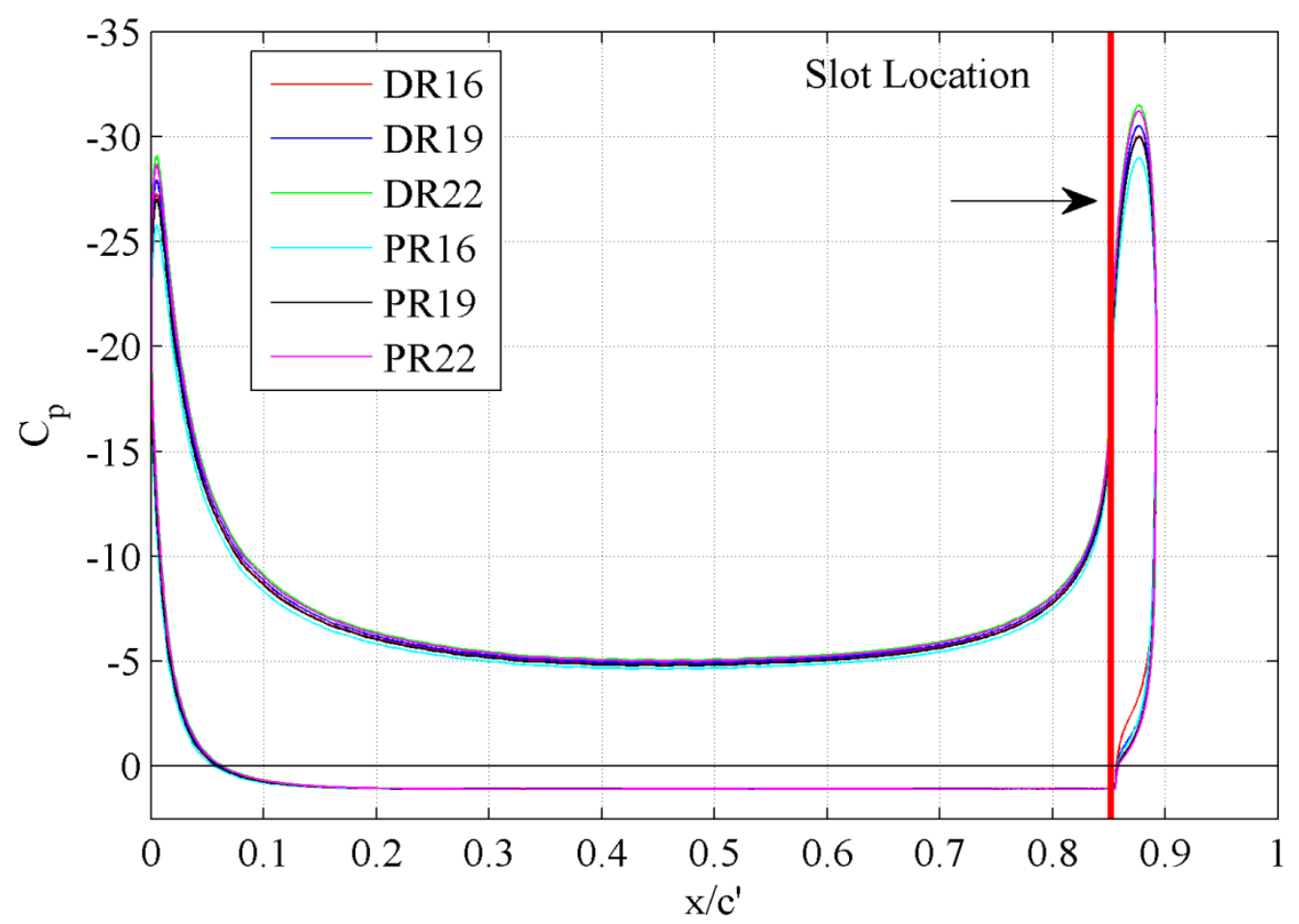

Figure 6-35: Pressure distributions for $\alpha=0^{\circ}, 90^{\circ}$ flaps

The above plot of $\mathrm{C}_{\mathrm{p}}$ shows the very significant suction peaks of the leading edge (-27.4) and trailing edge in the region of the slot (-30.2) revealing the rapid acceleration that the air must go through in order to follow the highly curved streamlines displayed in

Table 6-2. The leading edge suction peak $\mathrm{C}_{\mathrm{p}}$ increased a magnitude of $66 \%$ over the 
average leading edge suction peak of the $60^{\circ}$ flap deflections, and trailing edge peak increased a magnitude of 51\%. The cause of the significant negative pitching moment of the $90^{\circ}$ flap configurations can be seen in the pressure distribution where the aft suction peak surpasses the leading edge suction peak. Another observation is the $90^{\circ}$ deflection negative $C_{p}$ values do not extend as far aft as the $60^{\circ}$ deflection (Figure 6-7), due to the $90^{\circ}$ deflection rotating much of the flap surface under the existing airfoil. To further investigate the effect of each flap's geometry on the pressure coefficient peaks, a close-up view of both leading and trailing edge suction peaks are presented below in Figure 6-36 and Figure 6-37 respectively.

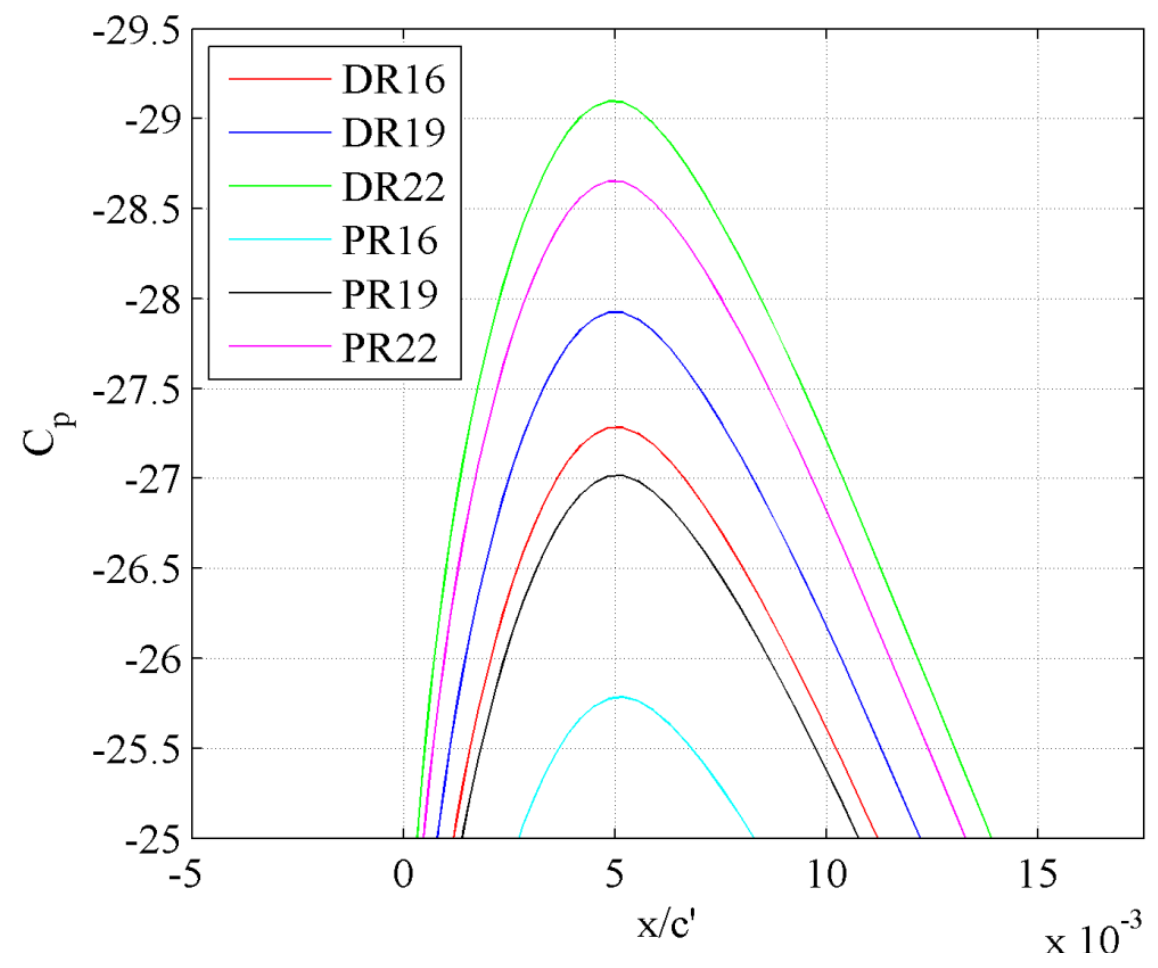

Figure 6-36: Leading edge suction peaks, $\alpha=0^{\circ}, 90^{\circ}$ flaps 


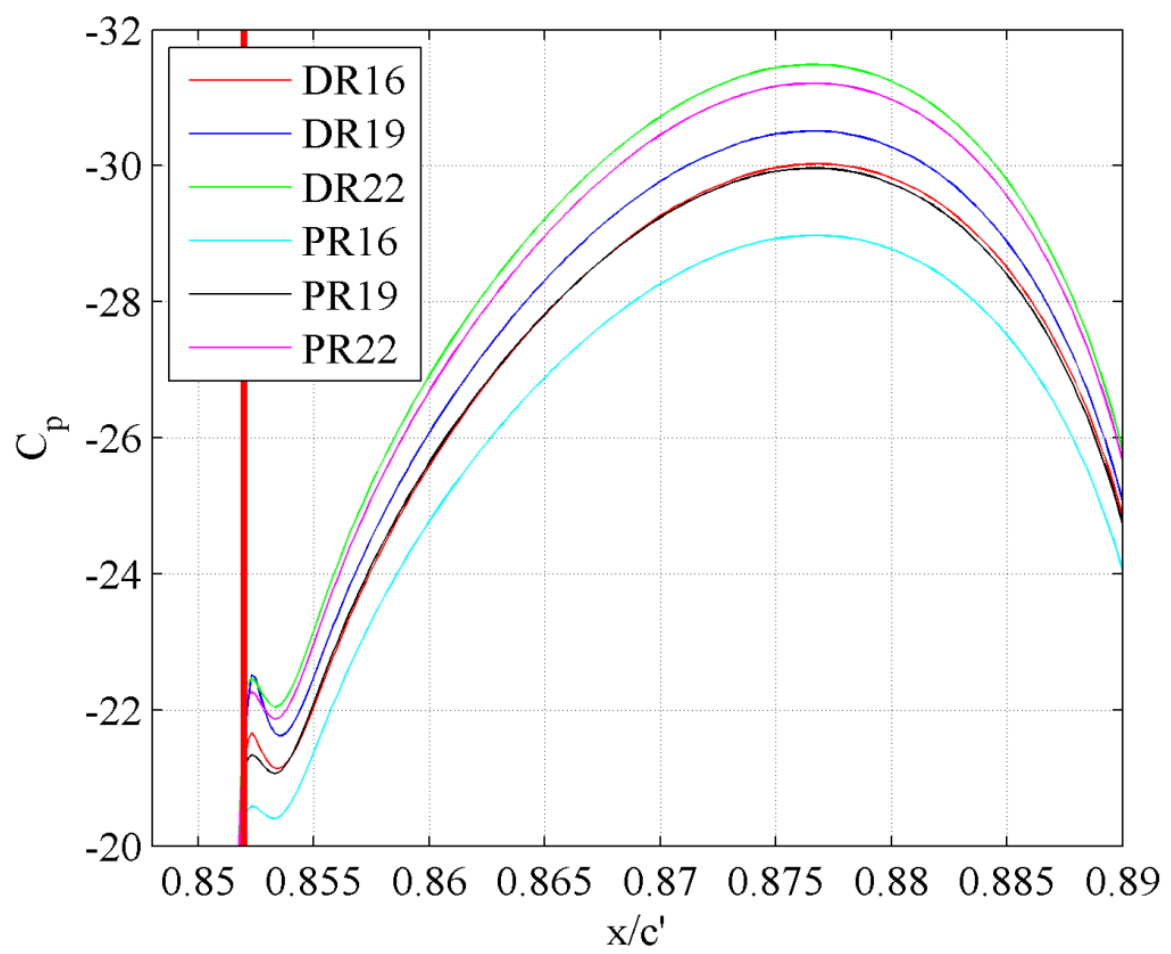

Figure 6-37: Trailing edge suction peaks, $\alpha=0^{\circ}, 90^{\circ}$ flaps

The leading edge suction peaks show a trend for the length of flap for each flap configuration, with the longer flaps reaching more negative $C_{p}$ values. Between the dual radius and prescribed radius configurations, the dual radius flaps produced the larger $C_{p}$ peaks. The same characteristics were observed for the trailing edge suction peak as well (Figure 6-37), with the longer 22\% flaps of each configuration attaining the larger suction peaks. DR22 produced the greatest value of $\mathrm{C}_{\mathrm{p}}$ of -29.1 for the leading edge suction peak, and -31.5 for the trailing edge peak, although produced less lift than both DR16 and DR19. An interesting note is that the highest lifting flap (DR16) did not obtain the largest pressure peaks at both leading edge and flap locations. All flaps experience the same sharp acceleration then deceleration just aft of the slot location as seen at the $60^{\circ}$ flap deflections, which is thought to be caused by the slot flow effective area expanding just aft of the slot. 
A detailed view of the $\mathrm{C}_{\mathrm{p}}$-distribution on the flap surfaces at the $90^{\circ}$ of deflection is presented below in Figure 6-38.

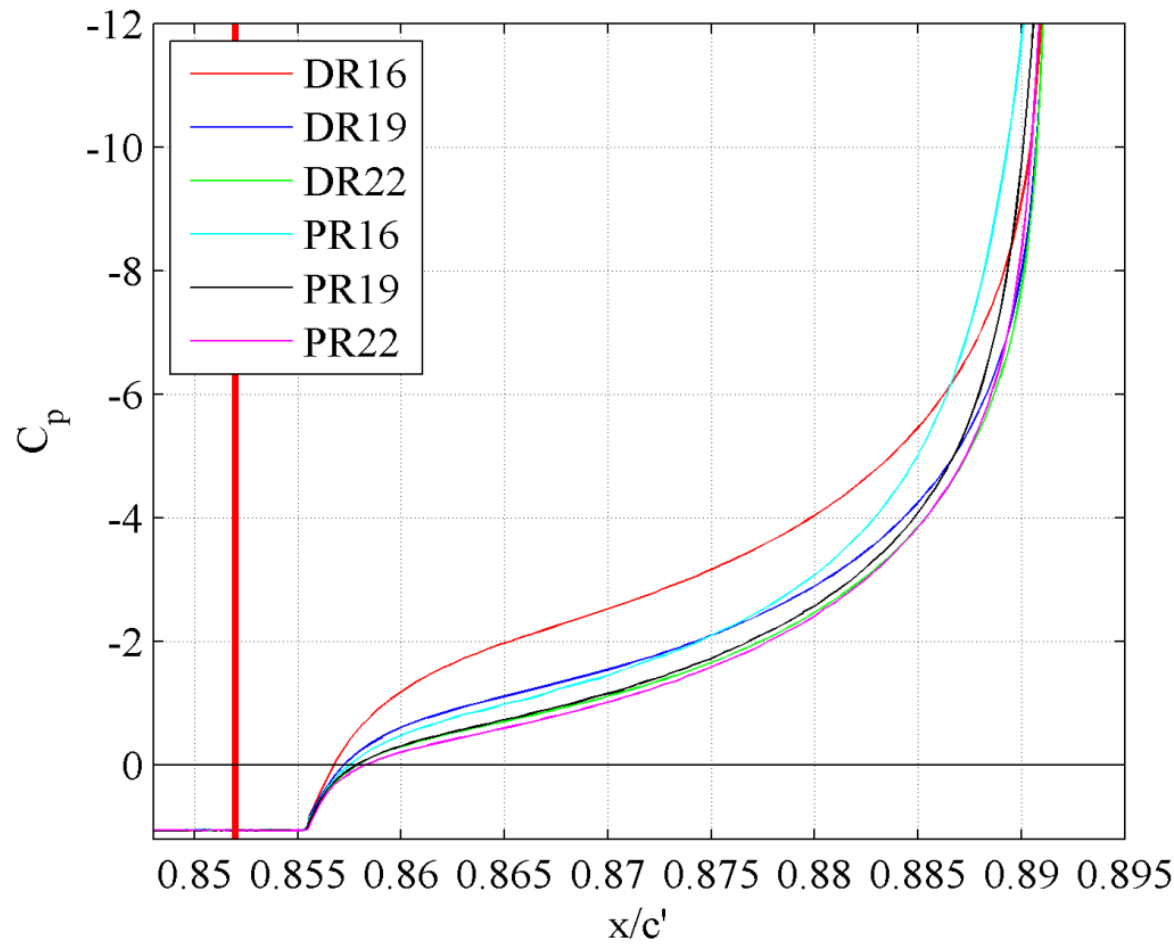

Figure 6-38: Flap surface pressure distributions, $\alpha=0^{\circ}, 90^{\circ}$ flaps

Due to $90^{\circ}$ of flap deflection, the pressure distributions of each secondary flap surface are displayed where $\mathrm{x} / \mathrm{c}^{\prime}$ travels from approximately 0.891 to 0.855 on Figure 6-38 above. The shorter flaps of each configuration carry more negative pressure on the surface, with the dual radius configurations having noticeably larger magnitudes for the shorter flap lengths. The $22 \%$ long dual radius and prescribed radius flaps display similar pressure distributions throughout this region of the airfoil. The $C_{p}$ values of each configuration also correspond to the amount of negative moment each flap produces.

The $\alpha=-5^{\circ}$ and $\alpha=3^{\circ} \mathrm{C}_{\mathrm{p}}$ plots for $90^{\circ}$ flap configurations are displayed below in Figure 6-39 and Figure 6-40, respectively, to determine the effects of $\alpha$ on the flaps. 


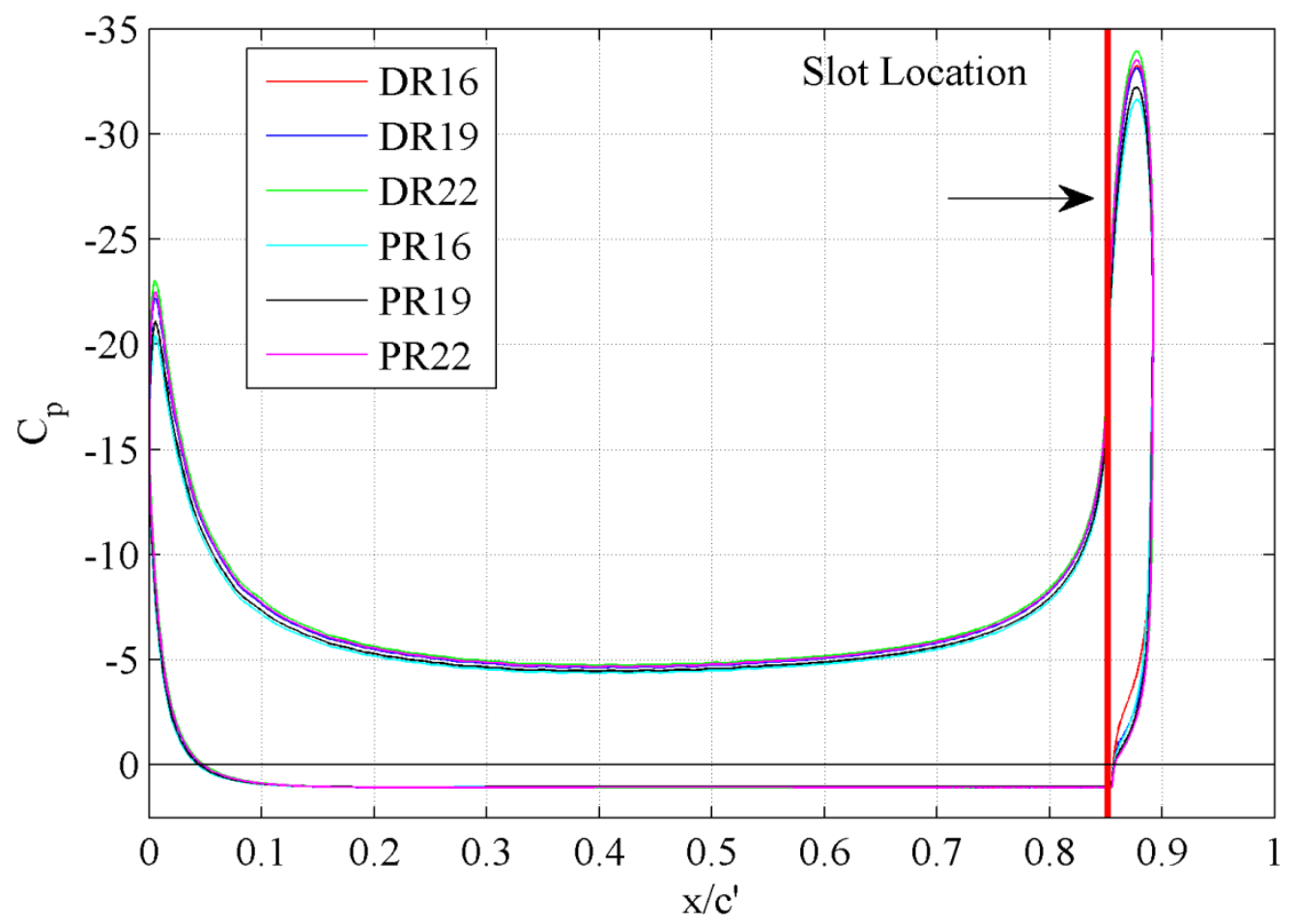

Figure 6-39: Pressure distributions for $\alpha=-5^{\circ}, 9^{\circ}$ flaps

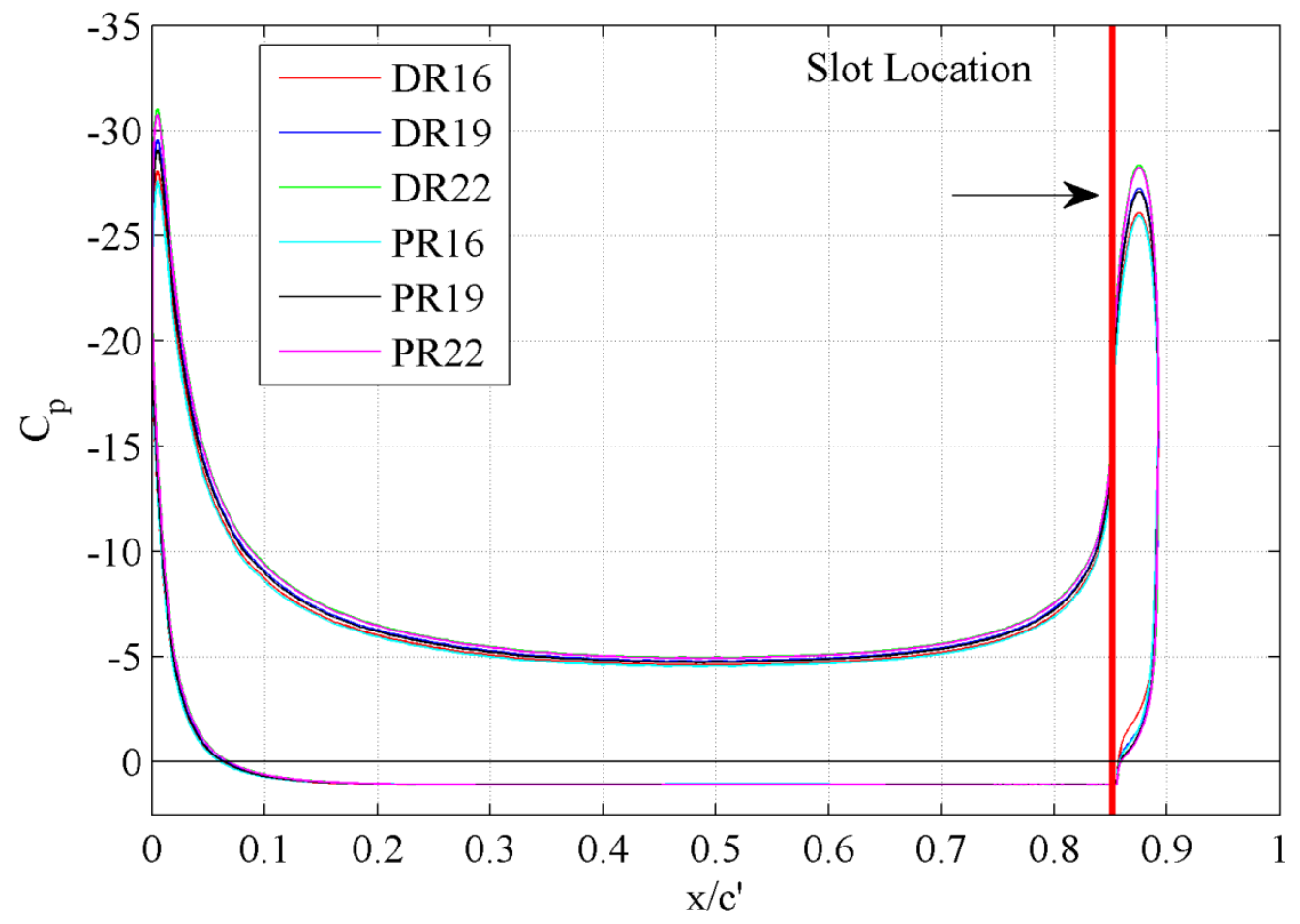

Figure 6-40: Pressure distributions for $\alpha=3^{\circ}, 9^{\circ}$ flaps 
The main changes of the $\alpha=-5^{\circ} \mathrm{C}_{\mathrm{p}}$ plot compared against the $\alpha=0^{\circ}$ plot, are the notably reduced leading edge suction peaks and the increased slot suction peaks. The average leading edge $C_{p}$ peaks dropped approximately $21 \%$ and the slot region $C_{p}$ peaks increased approximately $9 \%$ from $\alpha=0^{\circ}$ to $\alpha=-5^{\circ}$. These trends show the significant velocity increase over the leading edge with $\alpha$, and how the aft region/flap of the airfoil is more efficient at generating lift at negative $\alpha$ 's during the significant circulation caused by the $90^{\circ}$ flap deflection. Each of the flap configurations keep the same order of suction peak magnitudes as the $\alpha=0^{\circ}$ case, with the $22 \%$ flaps achieving the most negative values.

The $\mathrm{C}_{\mathrm{p}}$ distribution for the flaps at $\alpha=3^{\circ}$ show little increase in leading edge suction peak, and a significant drop in suction peak near the slot. The leading edge suction peaks increased an average of $7 \%$ and aft pressure peaks decreased an average of $10 \%$ from $\alpha=0^{\circ}$ to $\alpha=3^{\circ}$. Another observation that can be made from the $\alpha=3^{\circ} \mathrm{C}_{\mathrm{p}}$ plot is the more considerable effect that the flap length had on the $\mathrm{C}_{\mathrm{p}}$ values of the flaps. The differences between the flap lengths are better presented in the detailed $C_{p}$ plots at $\alpha=3^{\circ}$ of Figure 6-41 and Figure 6-42 below. From the lift plot of the flaps at $\alpha=3^{\circ}$ presented earlier (Figure 6-29), it appears that all configurations are experiencing characteristics of lift loss. From the two figures below, it is apparent that the longer flap configurations do not experience as significant of lift loss by keeping larger pressure peaks through $\alpha$ of $3^{\circ}$. 


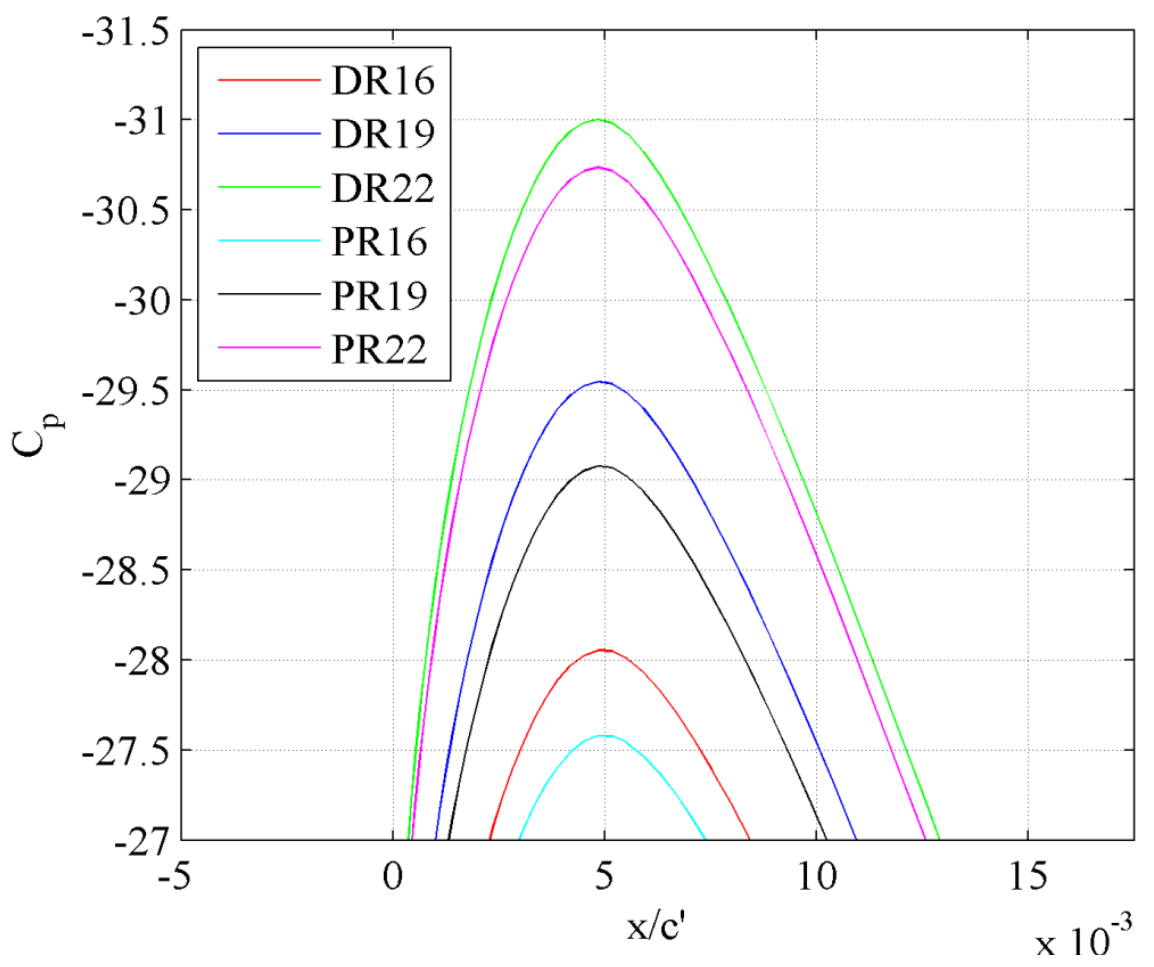

Figure 6-41: Leading edge suction peaks, $\alpha=3^{\circ}, 90^{\circ}$ flaps

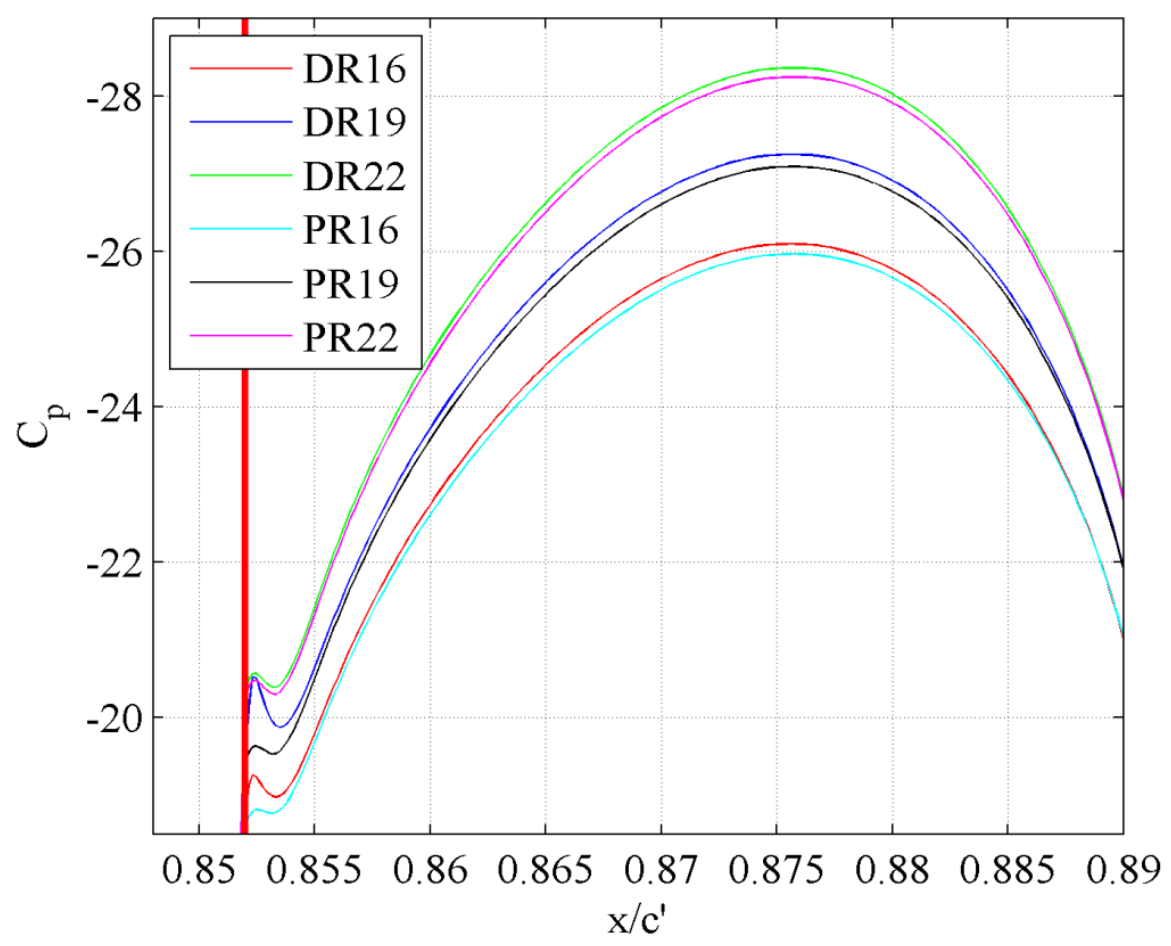

Figure 6-42: Trailing edge suction peaks, $\alpha=3^{\circ}, 90^{\circ}$ flaps 
Each airfoil's resulting $C_{f}$ for the approach/landing conditions at $\alpha=0^{\circ}$ is presented in Figure 6-43 below.

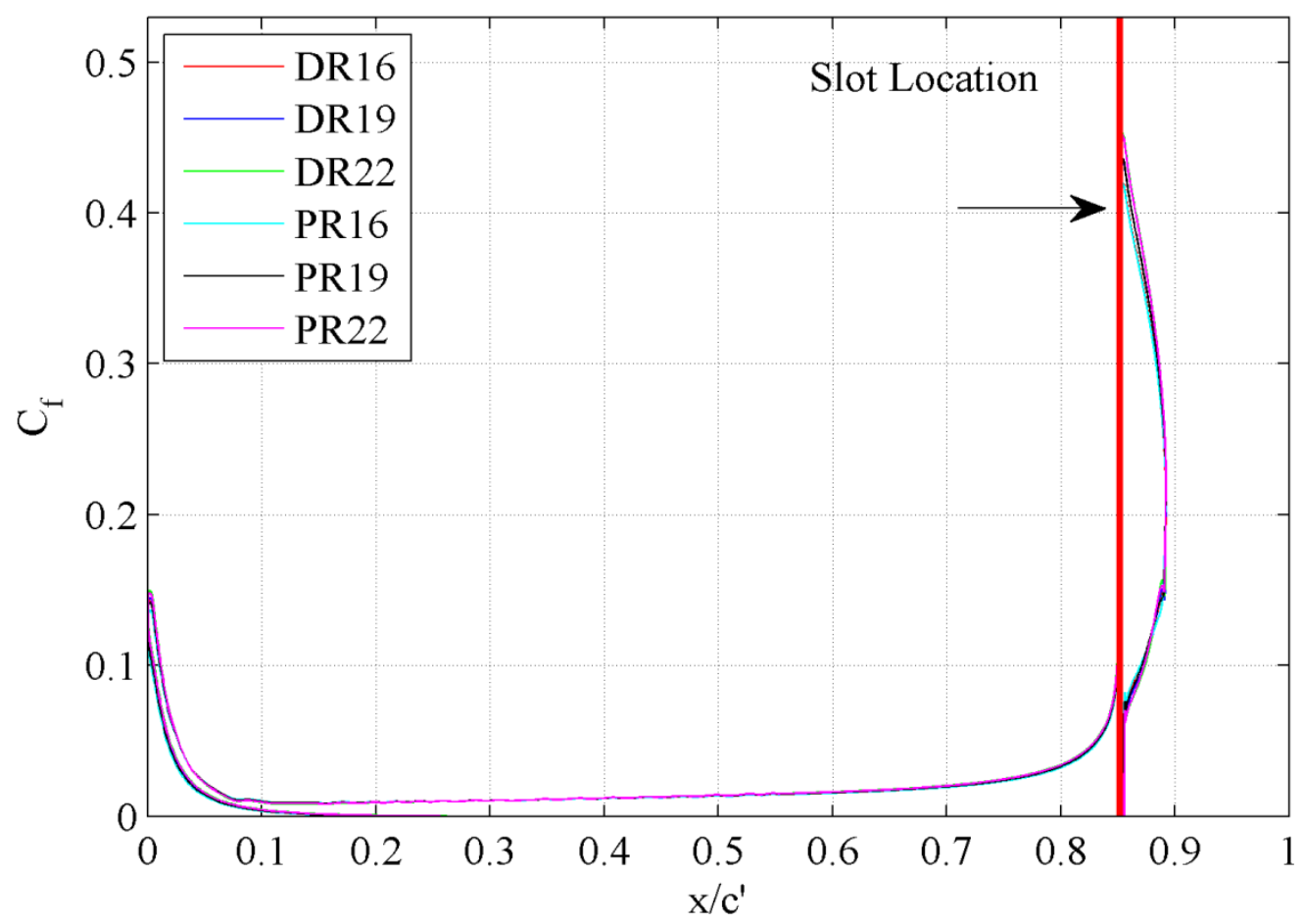

Figure 6-43: Skin friction distributions for $\alpha=0^{\circ}, 9^{\circ}$ flaps

Comparing this plot with that of the $60^{\circ}$ flap deflection (Figure 6-16), the main differences are the increased skin friction on the leading edge and slot location as well as a different distribution on the flap surface due to the geometrical deflection of the flap. The increased skin friction is a direct cause of the increase circulation and acceleration of flow in these regions. The leading edge skin friction peaks followed the same trends as the $\mathrm{C}_{\mathrm{p}}$ trends for each of the flaps, with the longer flaps topping each configurations $\mathrm{C}_{\mathrm{f}}$ values. The differences in $\mathrm{C}_{\mathrm{f}}$ aft of the slot and on the flap surface are detailed below in Figure 6-44. 


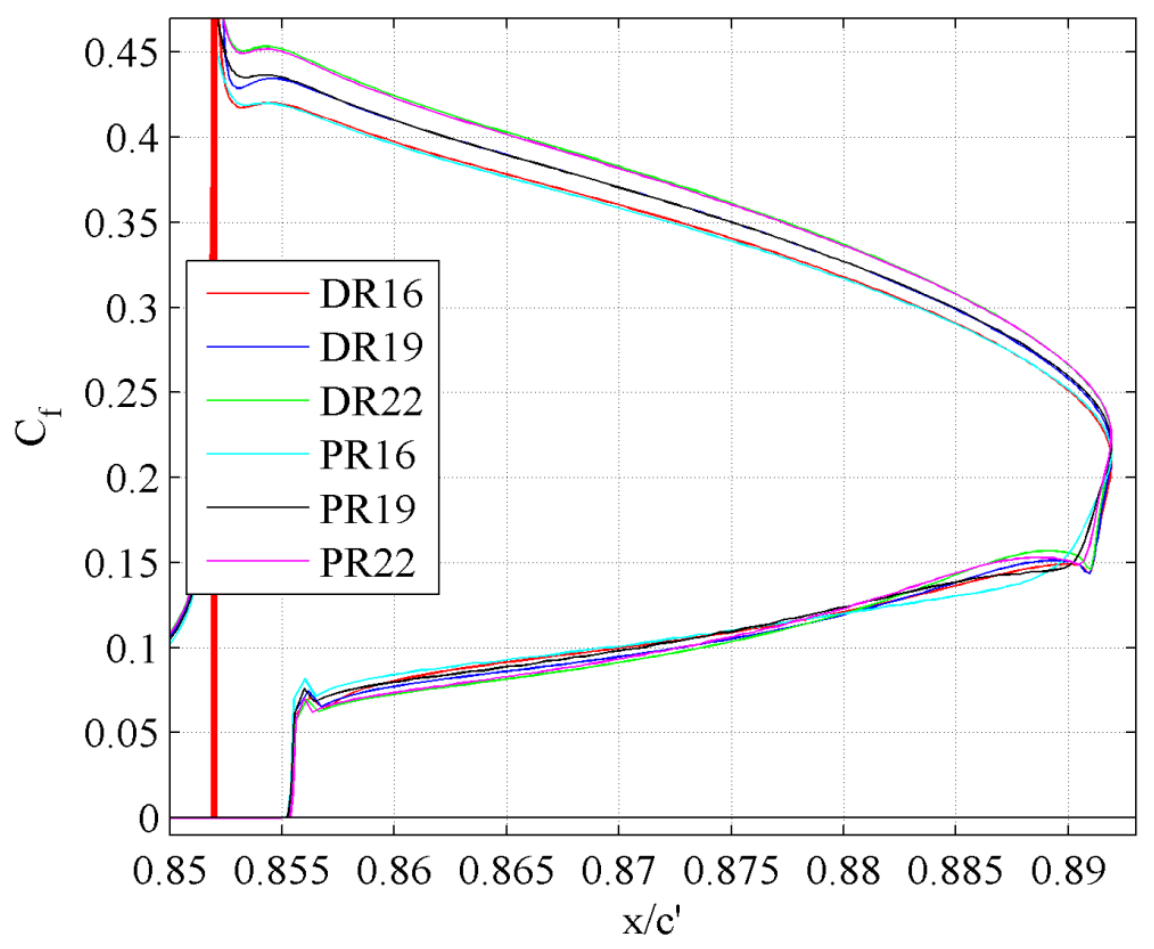

Figure 6-44: Flap surface skin friction distributions, $\alpha=0^{\circ}, 90^{\circ}$ flaps

Similar to the $60^{\circ} \mathrm{C}_{\mathrm{f}}$ data, all of the configurations encountered a small oscillation in $\mathrm{C}_{\mathrm{f}}$ shortly aft of the slot which was believed to be caused by the expansion of the slot flow effective area just aft of the slot accelerating the flow. The length of each of the flaps had the most effect on the $\mathrm{C}_{\mathrm{f}}$ value as the flow approached the furthest aft location, with the longer flaps sustaining the larger skin friction values or higher velocity flow over the flap surface. Once the flow turns past the furthest aft location on the flap and begins to travel forward in the $\mathrm{x}$-direction before it leaves the flap surface, the skin friction values of all the flaps converge. At an $\mathrm{x} / \mathrm{c}^{\prime}$ of approximately 0.891 , all of the flaps encountered the same "dip" or fluctuation in skin friction that was seen previously in the results of the $60^{\circ}$ flap deflections. The location of the "dip" corresponds to the point on the flap surface where $r_{1}$ transitions to either $r_{2}$ for the dual radius flaps or the cubically 
varying radius of the prescribed radius curve. Figure 6-45 below displays a larger view of the "dip" for further examination.

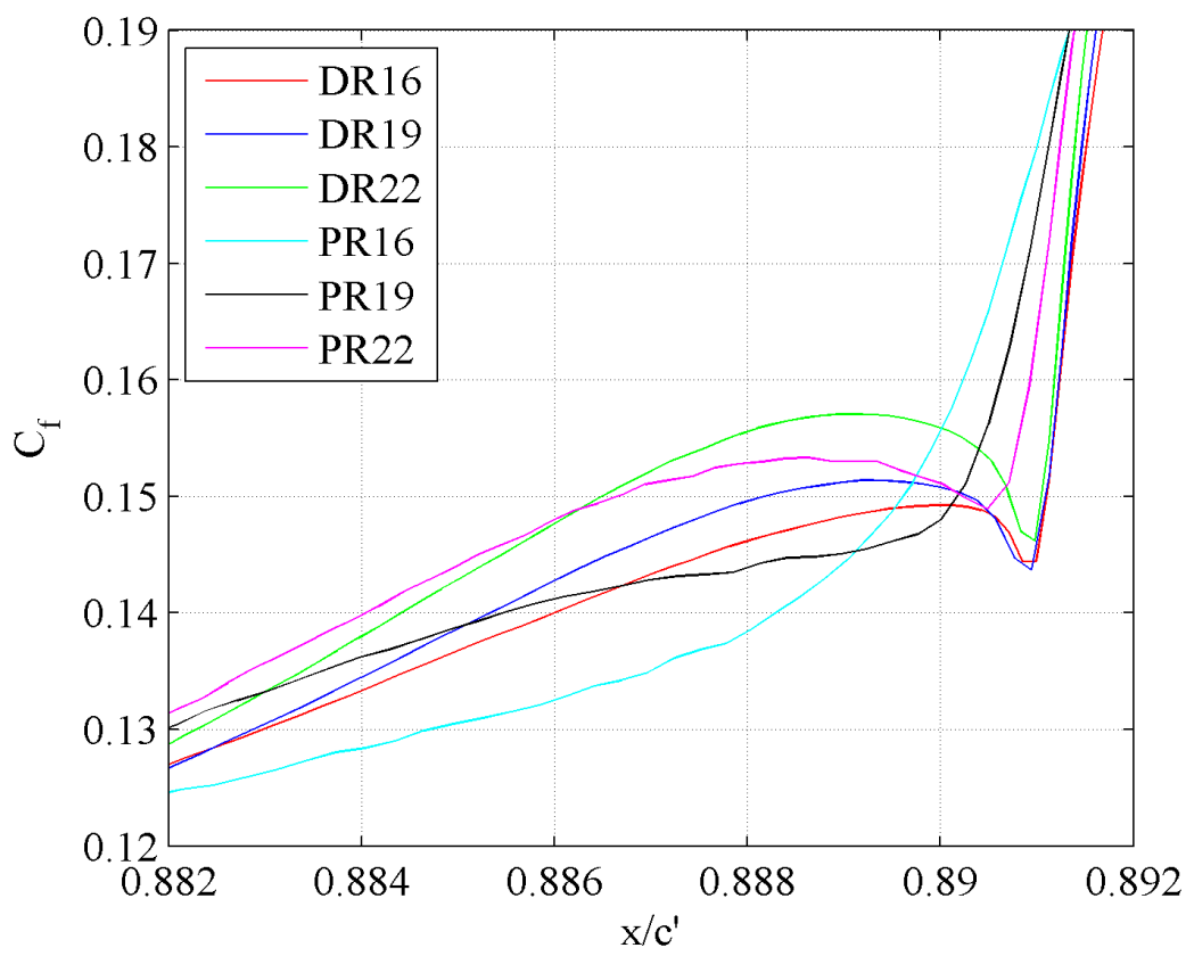

Figure 6-45: Detailed skin friction at flap surface transition, $\alpha=0^{\circ}, 90^{\circ}$ flaps

Figure 6-45 above shows how each of the dual radius flaps experienced an abrupt decrease then rise of skin friction, where the prescribed radius curves had a much more smooth transition in this region. Comparing the three prescribed radius flaps show the relationship of change in surface curvature to the severity of the skin friction "dip", with the smoother the curvature transitions experiencing less skin friction fluctuation. The PR16 configuration showed almost no signs of the change in surface geometry in terms of $\mathrm{C}_{\mathrm{f}}$. To compare the different flaps' surface curvature in the location of this "dip" refer to the curvature plot presented in the $60^{\circ}$ flap deflection results above in Figure 6-19. It is suggested that the flaps with the smoother curvature transition encourage flow 
attachment by not encountering the sudden deceleration then acceleration of the flow, which is shown by the skin friction "dip".

The $\mathrm{C}_{\mathrm{f}}$ plots for all the flap configurations at $\alpha=-5^{\circ}$ and $\alpha=3^{\circ}$ stayed consistent with the $\alpha=0^{\circ}$ plot with only slight variation of magnitude as seen with $C_{p}$, and have been omitted from the results section. Note that these plots are located in appendix for reference.

The wall $\mathrm{y}^{+}$values for the $90^{\circ}$ flap deflection simulations at $\alpha=0^{\circ}$ are presented below in Figure 6-46.

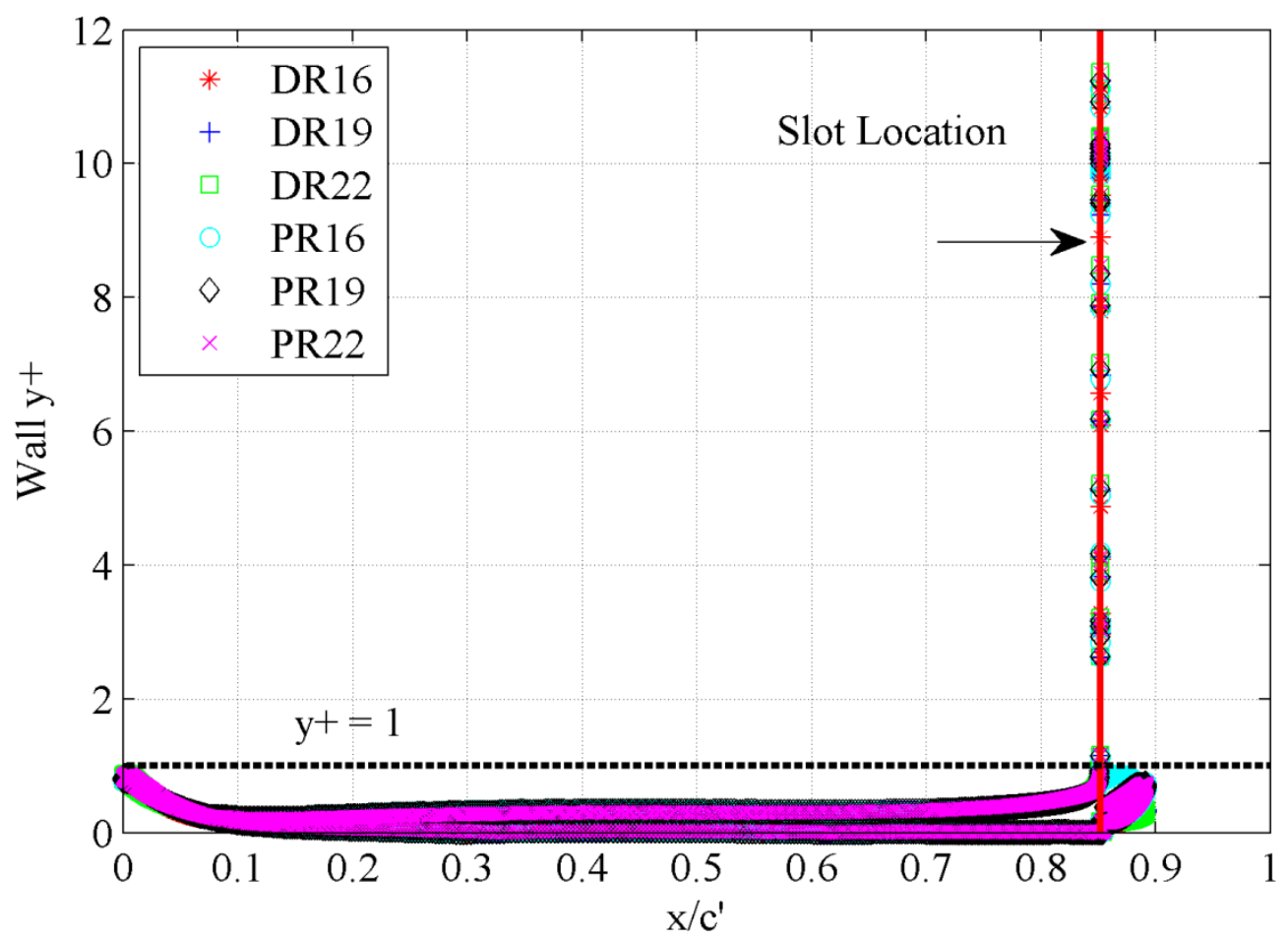

Figure 6-46: $y^{+}$values of the airfoil/flap configurations, $\alpha=0^{\circ}, 90^{\circ}$ flaps

The $\mathrm{y}^{+}$values stayed below one for all the flap configurations investigated, with the exception of the slot location. These values were considered adequate for the simulation's solving methods. The $\mathrm{y}^{+}$values of the $\alpha=-5^{\circ}$ and $\alpha=3^{\circ}$ cases also stayed 
below one, and displayed the same traits as the $\alpha=0^{\circ}$ results. Plots of $\mathrm{y}^{+}$for the $\alpha=-5^{\circ}$ and $\alpha=3^{\circ}$ cases are shown within the appendix.

\section{Flap Parameter Trends}

To examine the influence of the flap parameters on approach/landing performance $\left(90^{\circ}\right.$ flap deflection), the performance parameters of $\mathrm{C}_{\mathrm{l}}, \mathrm{C}_{\mathrm{d}}$, and $\mathrm{C}_{\mathrm{m}}$, (c/4) were plotted against the flap's geometric parameters. The reasons for tracking these three performance parameters was the requirement of high lift and drag with minimal pitching moment to allow the aircraft to approach and land using the steeper approach angles desired by the NASA CESTOL goals, while not requiring a significantly large tail to counter pitching moments of this mission segment. The main interest of the approach/landing setting was to maximize $\mathrm{C}_{1}$ and $\mathrm{C}_{\mathrm{d}}$ performance parameters while

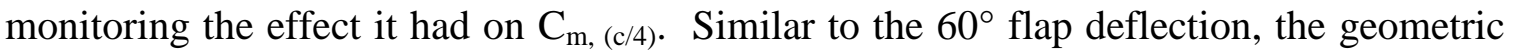
parameters that were investigated were the flap's departure angle $(\theta)$, the flap chord-toairfoil chord ratio $\left(\mathrm{c}_{\mathrm{f}} / \mathrm{c}^{\prime}\right)$, and the radius ratio $\left(\mathrm{r}_{2} / \mathrm{r}_{1}\right.$ or $\left.\mathrm{r}_{2 \_ \text {avg }} / \mathrm{r}_{1}\right)$. Although each geometric parameter is dependent upon the other parameters, each parameter was analyzed separately in attempt to determine each parameter's individual influence on the approach/landing performance parameters studied. Note that all flap performance was analyzed at $\alpha=0^{\circ}$.

Figure 6-47, Figure 6-48, and Figure 6-49 below display the approach/landing performance trends with variation of the flap's departure angle $(\theta)$. 


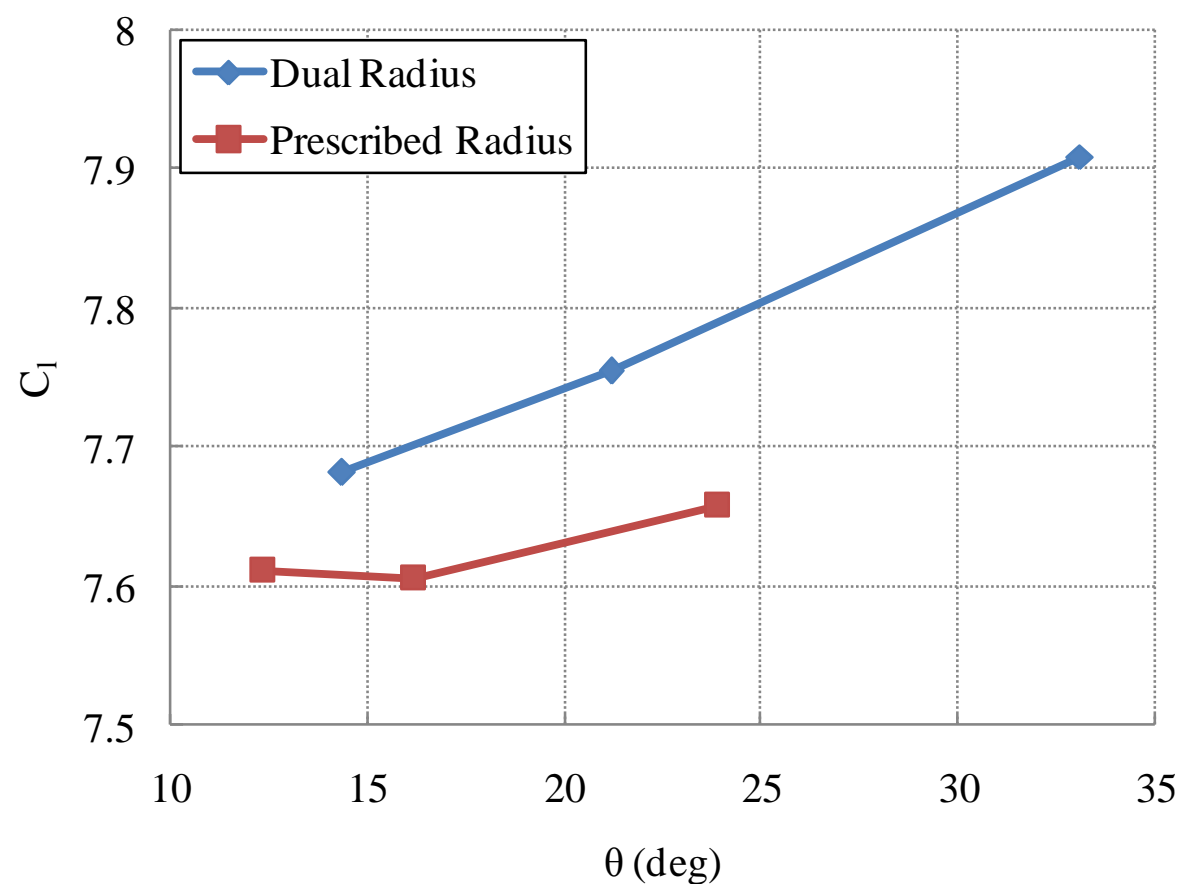

Figure 6-47: Lift for flap configurations given $\theta$

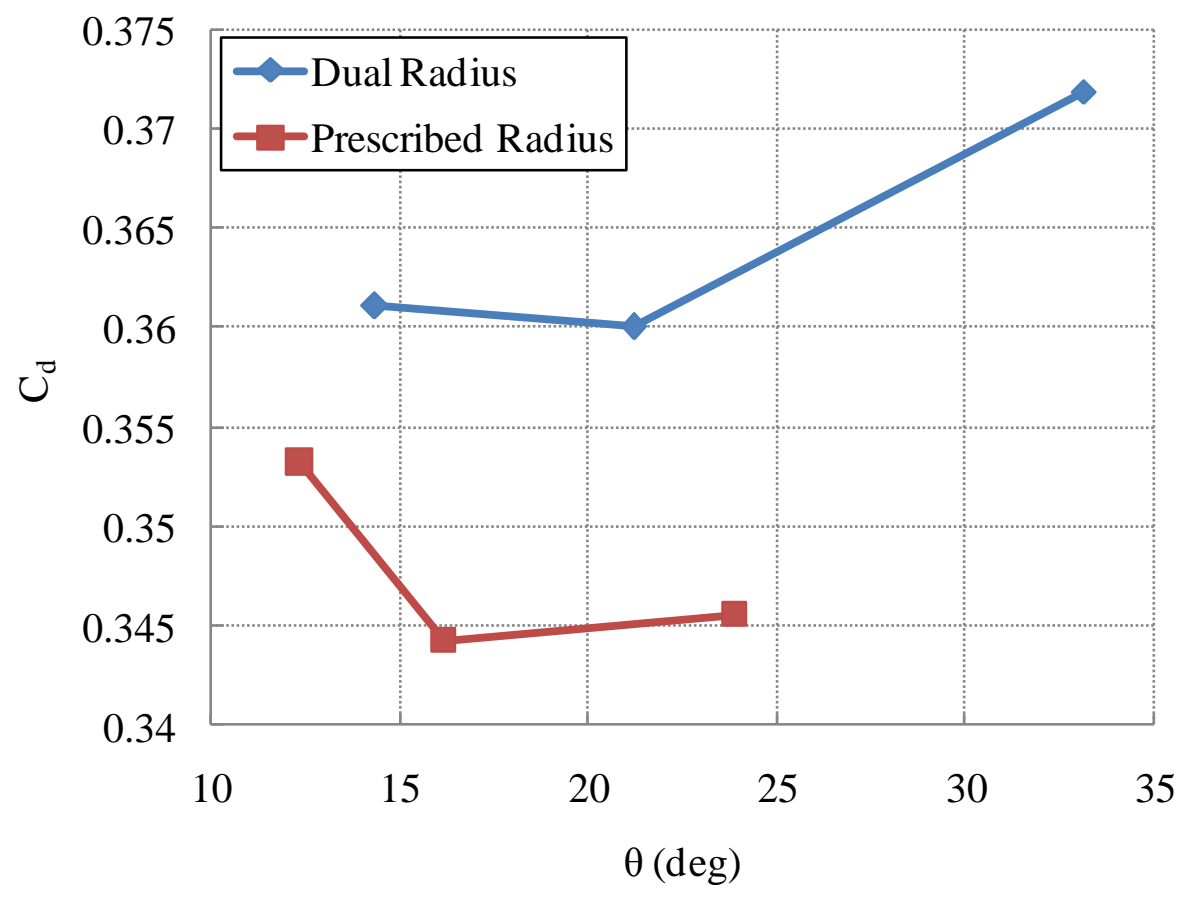

Figure 6-48: Drag for flap configurations given $\theta$ 


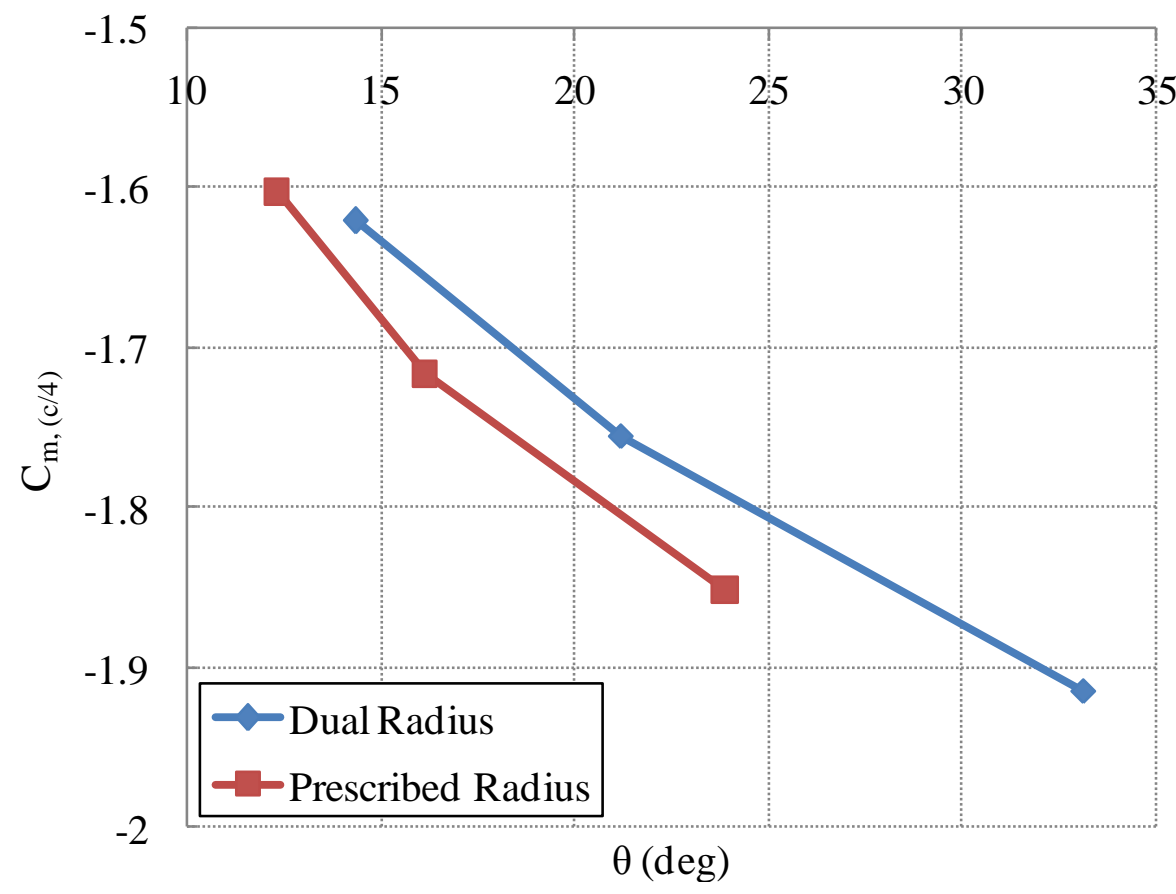

Figure 6-49: Pitching moment for flap configurations given $\theta$

Observing the above lift performance trends, there is a clear advantage of larger $\theta$ values producing greater lift for both flap configurations. The $\mathrm{C}_{\mathrm{d}}$ data displays, again, similar behavior of both configurations decreasing, and then increasing in drag with increasing $\theta$. The prescribed radius flap reaches its highest drag value at lower $\theta$ values, where the dual radius accomplishes its highest drag at the larger $\theta$ values. When considering both lift and drag trends of the $90^{\circ}$ flap configurations, the larger $\theta$ values offer the more desirable performance characteristics of achieving both large lift and drag values.

Figure 6-49 above displays the negative pitching moment trends, showing the rapid increase in moment with $\theta$. These trends resulted in the dual radius configuration experiencing a slightly less negative pitching moment given $\theta$. With pitching moment 
significantly increasing with $\theta$, it suggests a flap configuration yielding the largest lift and drag given a low $\theta$ value will provide the best approach/landing performance.

Figure 6-50, Figure 6-51, and Figure 6-52 below depict the same approach/landing performance trends given the flap chord-to-modified airfoil chord length ratio $\left(\mathrm{c}_{\mathrm{f}} / \mathrm{c}^{\prime}\right)$.

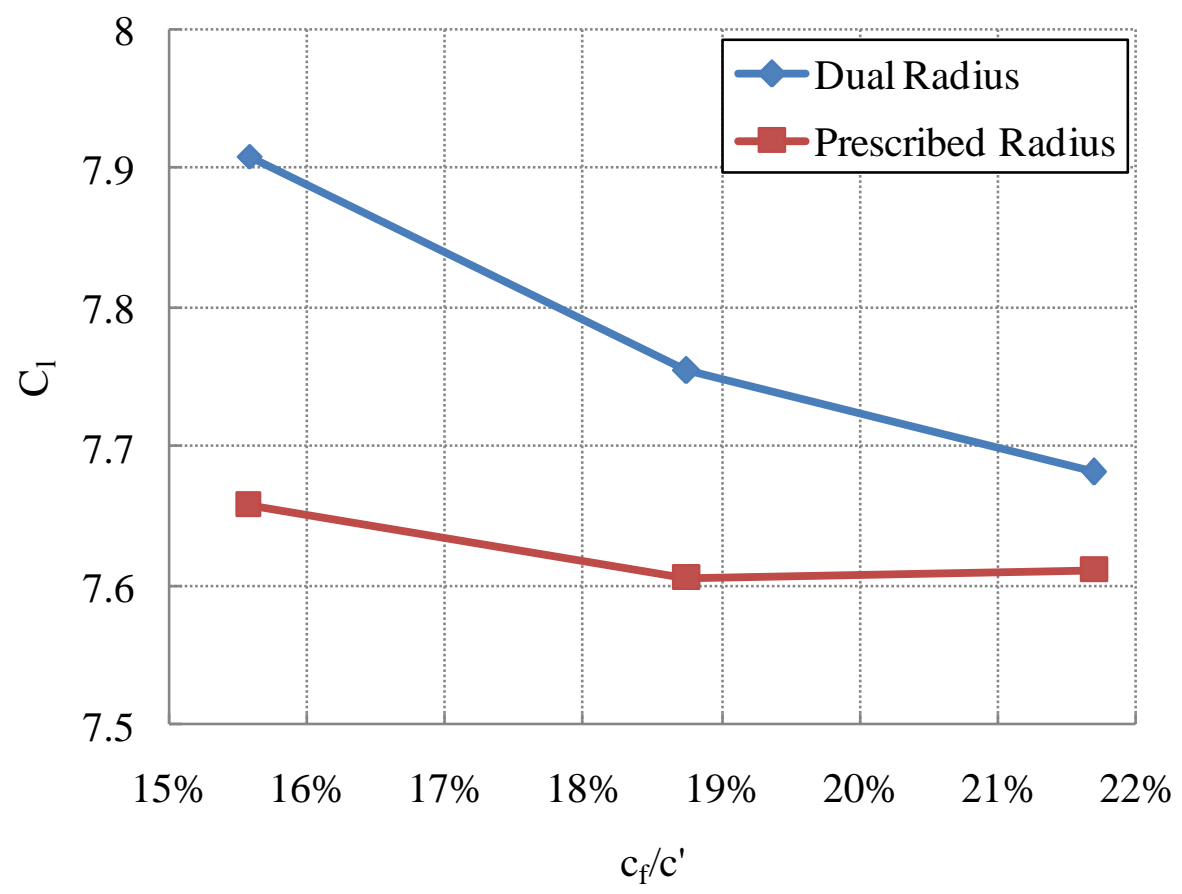

Figure 6-50: Lift for flap configurations given $c_{f} / c^{\prime}$ 


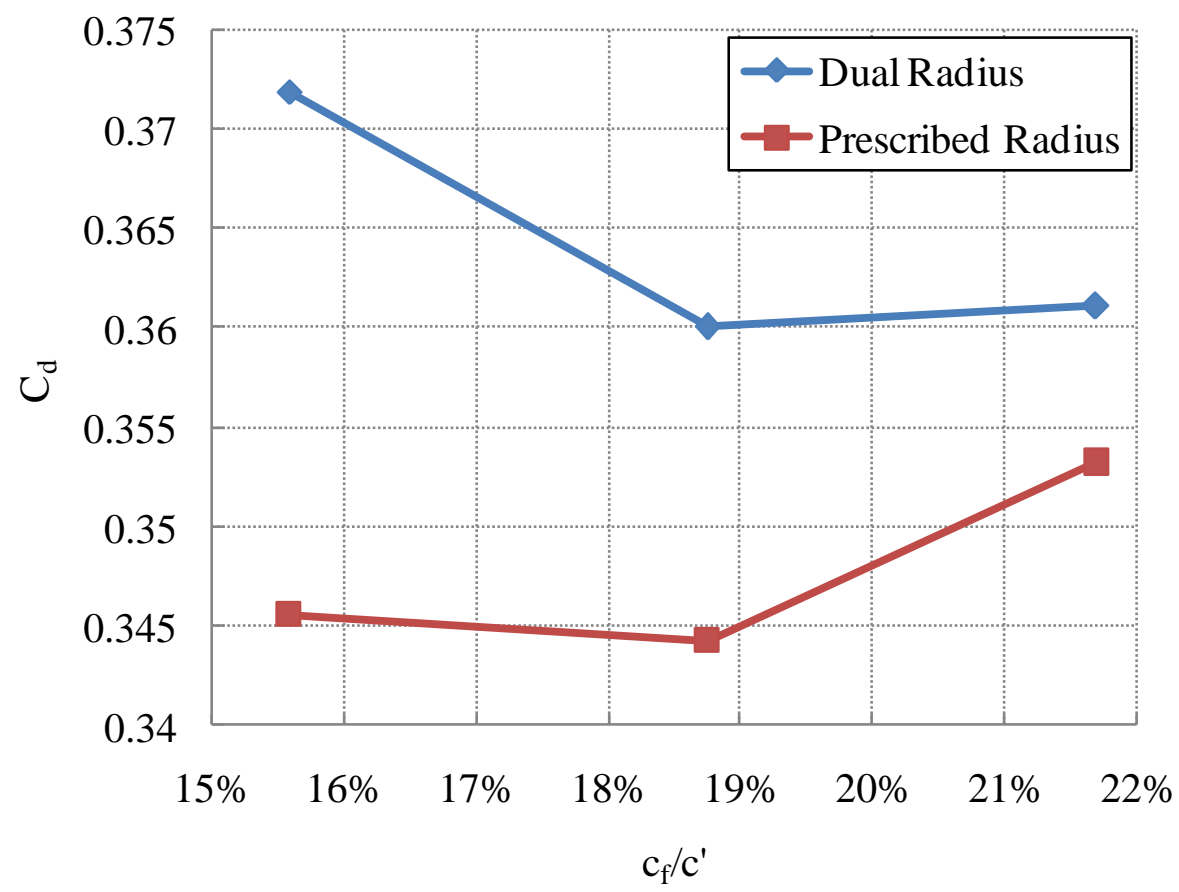

Figure 6-51: Drag for flap configurations given $c_{\mathrm{f}} / \mathrm{c}^{\prime}$

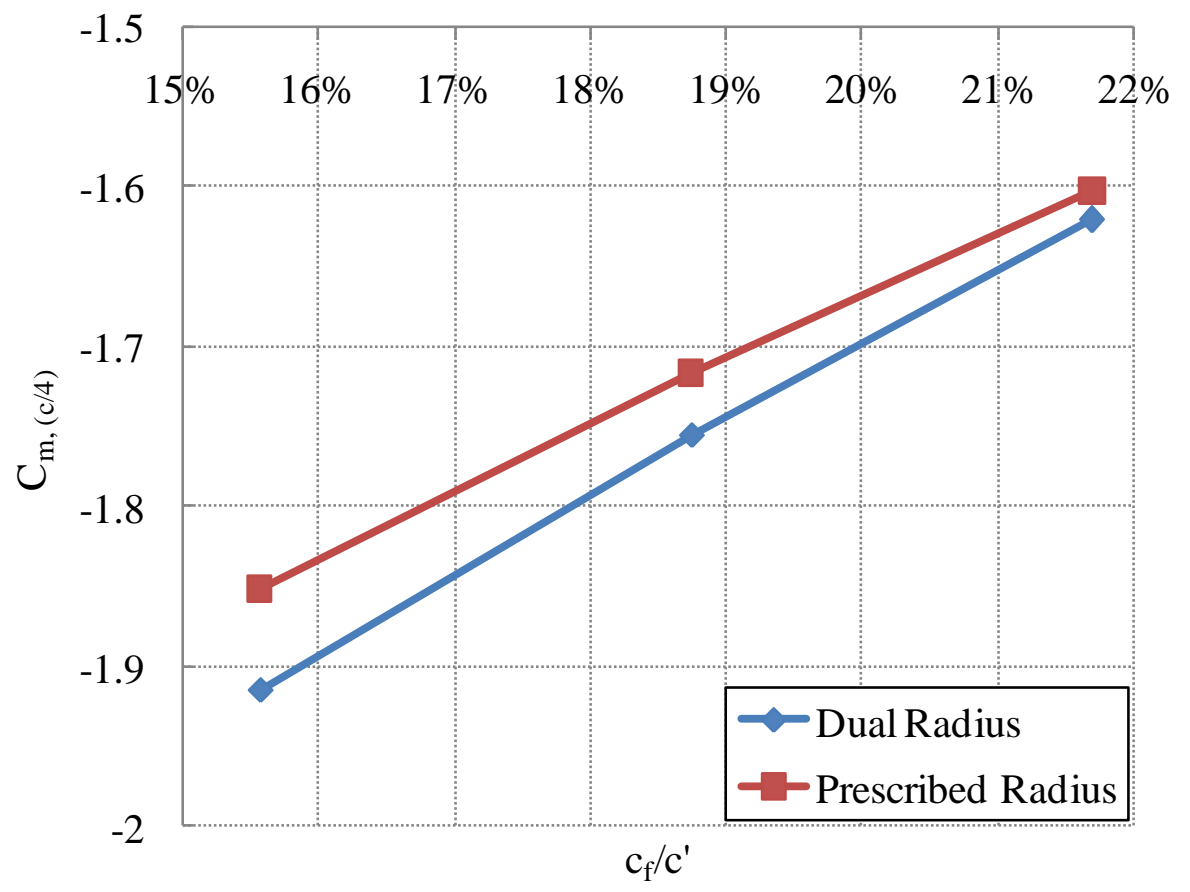

Figure 6-52: Pitching moment for flap configurations given $c_{\mathrm{f}} / \mathrm{c}^{\text {' }}$ 
All of the flap configurations showed a slight decrease in lift as the flap length increased, with the dual radius experiencing a larger drop than prescribed radius flaps. The drag data demonstrated a similar relation to the $\theta$ trends, with an initial drop in drag following by an increase at the $22 \%$ flaps. The dual radius flaps attained its maximum drag at the shorter flap lengths, where the prescribed did at the longer flap lengths. The lift and drag data displayed both of the configurations offering beneficial approach/landing performance (high lift and drag) at either end of the $\mathrm{c}_{\mathrm{f}} / \mathrm{c}^{\prime}$ geometric parameter.

The $\mathrm{C}_{\mathrm{m}}$, (c/4) data experienced almost linear trends, with the prescribed radius configurations maintaining a consistently lower magnitude negative pitching moment over the range of flap lengths studied. Both configurations exhibited a constant reduction in negative pitching moment with increase in flap length, pointing to a significant benefit of the longer flaps. When considering each flap configurations lift and drag performance with the pitching moment data, it demonstrates the advantages of the prescribed radius flap maintaining its high lift and drag at the longer flap lengths resulting in the best pitching moment of the presented data.

Figure 6-53, Figure 6-54, and Figure 6-55 below display the approach/landing performance trends and their respective equations for the radius ratio $\left(r_{2} / r_{1}\right)$ of the dual radius flaps. The prescribed radius ratio $\left(\mathrm{r}_{2}\right.$ avg $\left./ \mathrm{r}_{1}\right)$ performance trends are shown in Figure 6-56, Figure 6-57, and Figure 6-58. Note that the dual radius and prescribed radius configurations were plotted separately due to the difference in $r_{2} / r_{1}$ to $r_{2 \_a v g} / r_{1}$ magnitudes. 


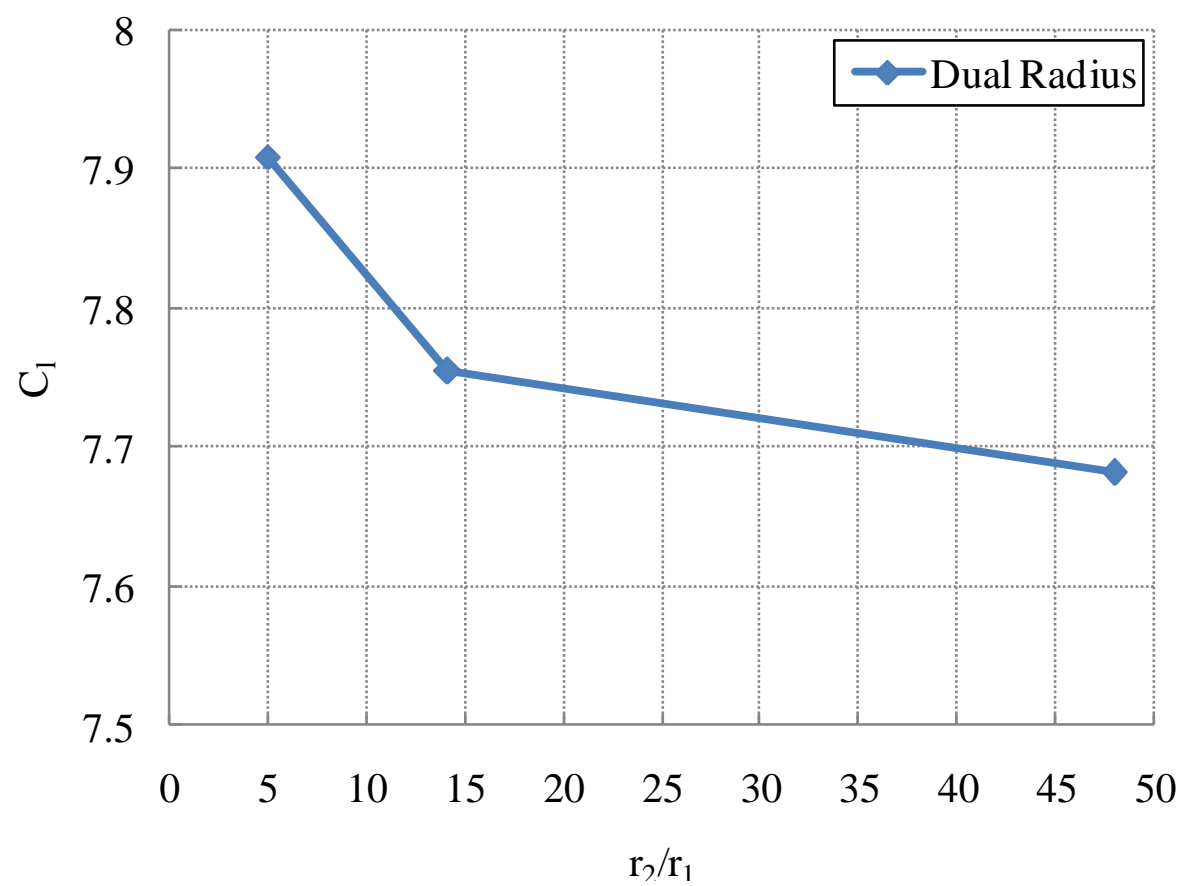

Figure 6-53: Lift for dual radius given $r_{2} / \mathbf{r}_{1}$

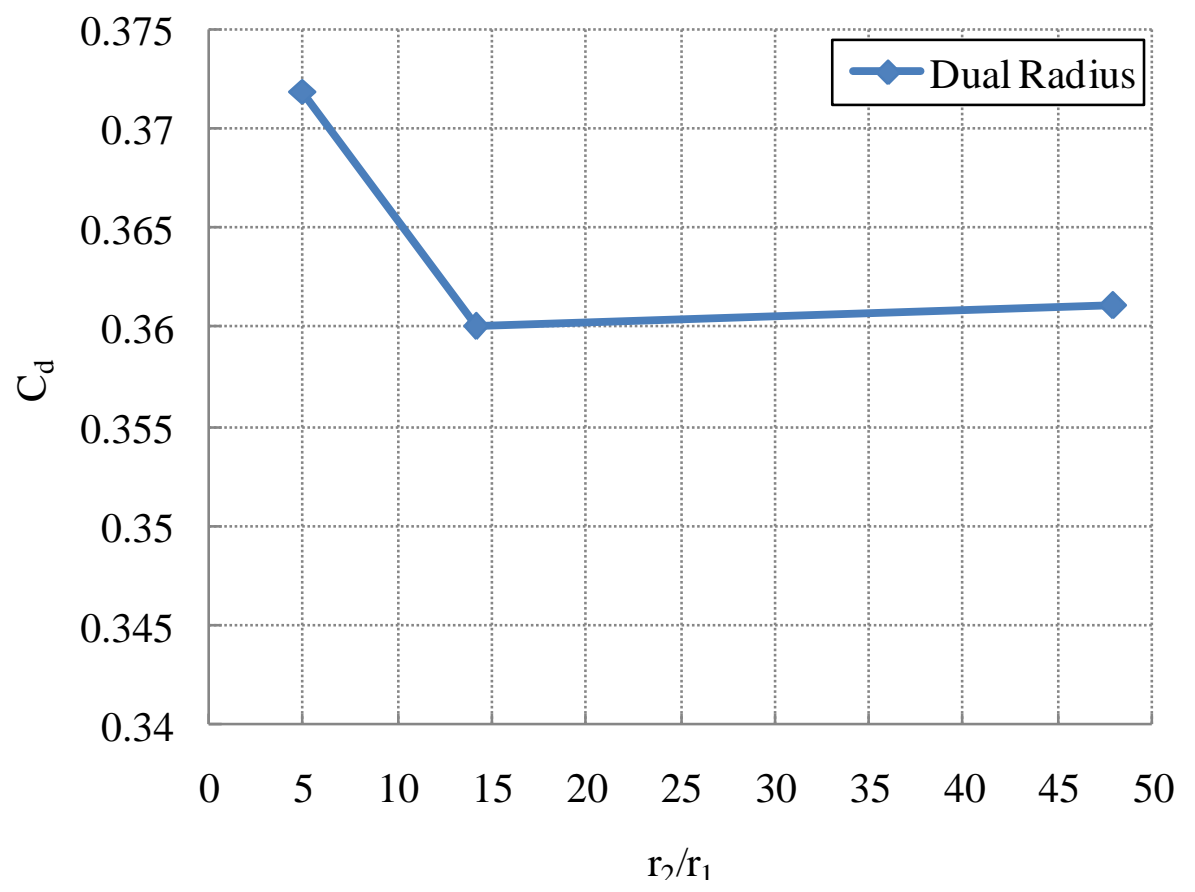

Figure 6-54: Drag for dual radius given $r_{2} / \mathbf{r}_{1}$ 


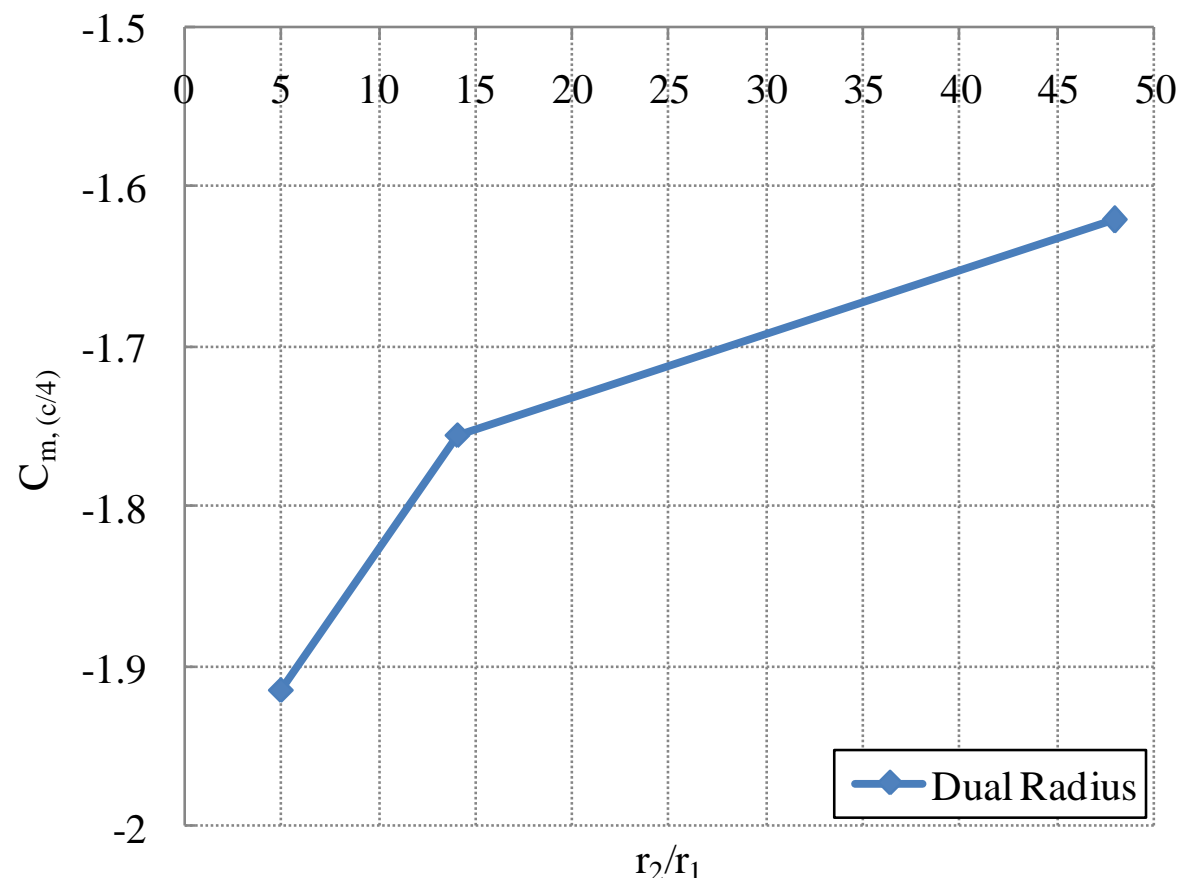

Figure 6-55: Pitching moment for dual radius given $\mathbf{r}_{2} / \mathbf{r}_{1}$

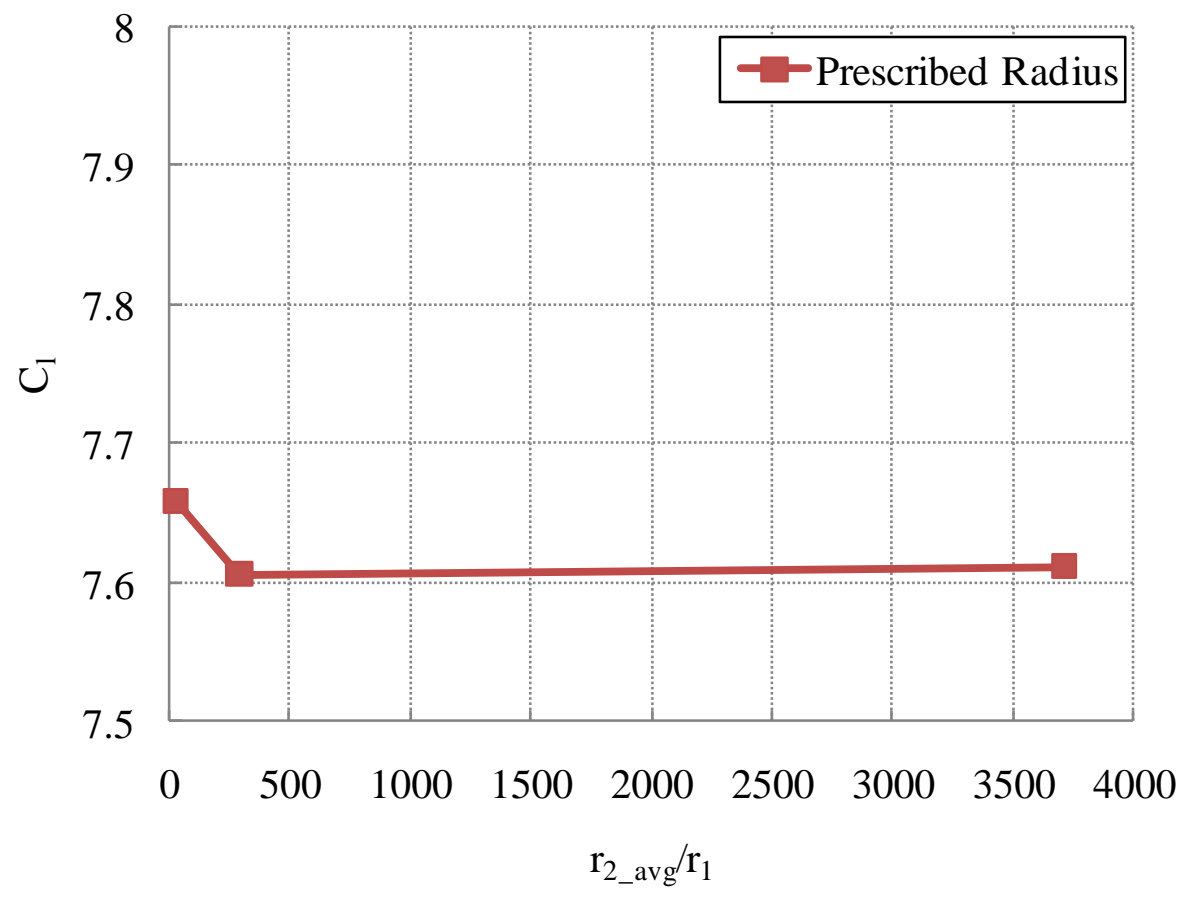

Figure 6-56: Lift for prescribed radius given $\mathbf{r}_{2 \_a v g} / \mathbf{r}_{1}$ 


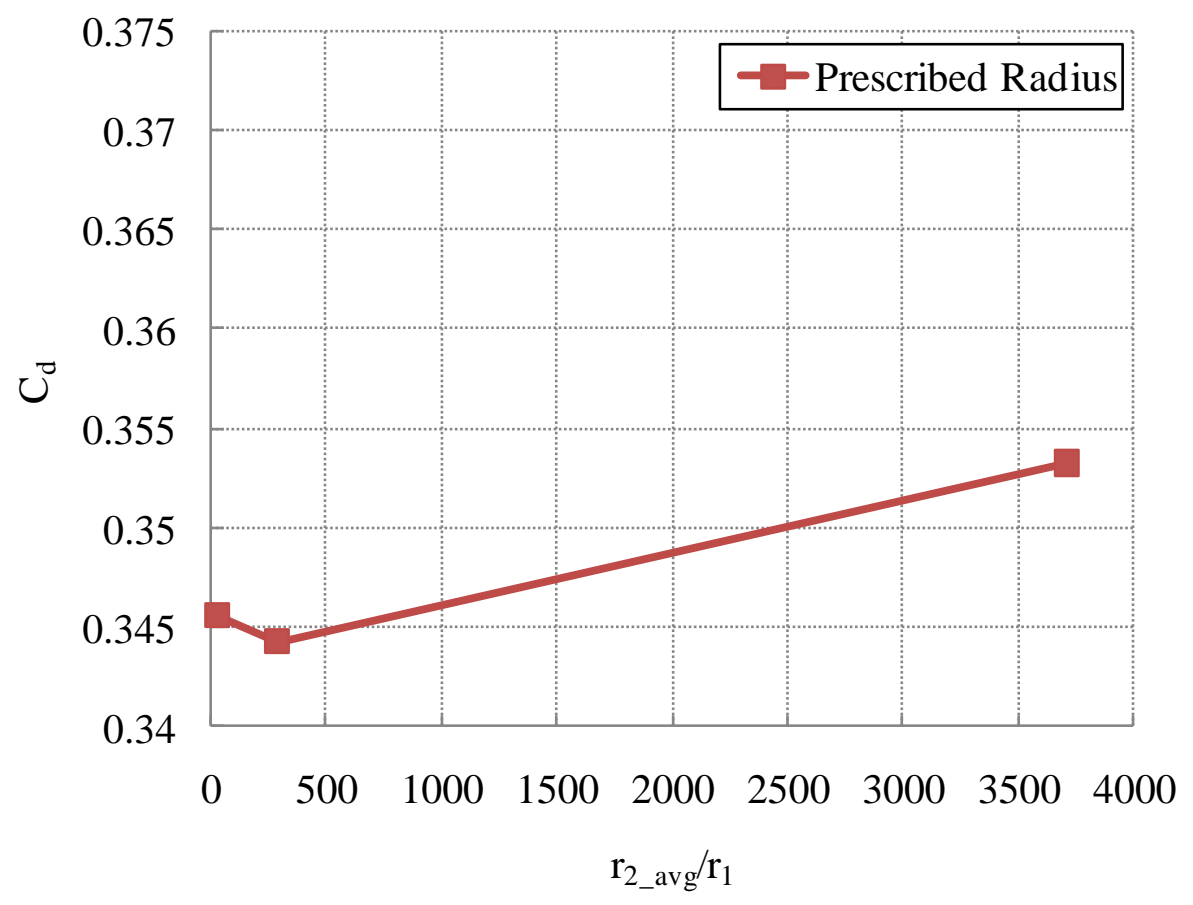

Figure 6-57: Drag for prescribed radius given $\mathbf{r}_{2 \_a v g} / \mathbf{r}_{1}$

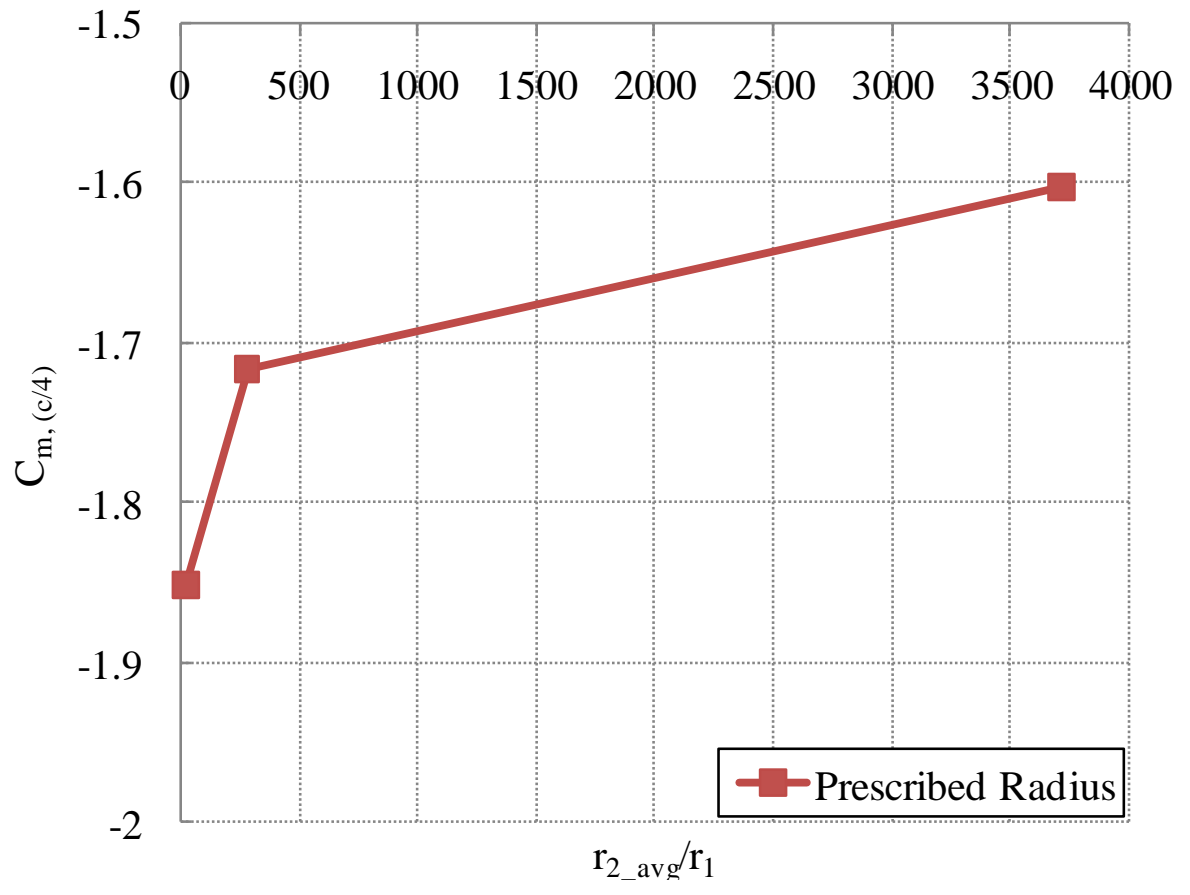

Figure 6-58: Pitching moment for prescribed radius given $\mathbf{r}_{2 \_ \text {avg }} / \mathbf{r}_{1}$ 
The two different flap configurations displayed very similar trends for all the approach/landing performance parameters, with the prescribed radius configurations experiencing more extreme behavior due to the spacing of data points along the $r_{2 \_a v g} / r_{1}$ geometric parameter. The lift trends with radius ratios of both flap configurations initially decreased as radius ratios increased. Although as the dual radius flap's lift continued to drop with more radius ratio, the prescribed radius flap's lift slightly increased. This suggested that the prescribed radius configurations might be capable of additional lift performance at larger radius ratios. Again, due to the skewed radius ratio of the prescribed radius, it is recommended to refine the trend with additional data points at intermediate $r_{2 \_a v g} / r_{1}$ values.

The drag performance trends were similar in showing an initial drop, then increase as radius ratio increased. Although the dual radius reaches its maximum drag at the lowest radius ratios, the prescribed radius experienced its largest drag at the largest ratios. The trends imply the smaller radius ratios of the dual radius would be the most desirable, where the prescribed radius displayed a clear advantage of the larger ratios. It should be noted, that the dual radius drag maintained larger magnitudes over all of the prescribed radius configurations.

The pitching moment data displayed very similar trends to both flap configurations, with the moment following a logarithmic shape as radius ratio increased. Again, the prescribed radius configuration maintained a lower negative pitching moment than that of the dual radius. Similar to the previous trends of $\theta$ and $\mathrm{cf} / \mathrm{c}$ ', the radius ratios portray the prescribed radius flap maintaining the best approach/landing performance with its highest drag, large lift, and smallest moment occurring at the largest radius ratio. 


\section{Cruise Performance - Transonic Airspeed, $0^{\circ}$ Flaps}

With the cruise performance of an airfoil being one of the most important characteristics of its design, the impact of the different flap geometries were examined at the transonic conditions of AMELIA's intended cruise of 0.74 Mach. The simulation of cruise involved first running each airfoil/flap configuration at two $\alpha$ 's to obtain their respective 2-D lift-curve, then solving the geometries at their appropriate $\alpha$ to obtain the target 0.247 3-D cruise $\mathrm{C}_{\mathrm{L}}$ of AMELIA's design. Applying the 3-D lift curve approximation [equation (40)] to the target cruise $\mathrm{C}_{\mathrm{L}}$ corresponded to a target 2-D $\mathrm{C}_{1}$ of 0.320. Table 6-3 below displays the Mach contours and required $\alpha$ for all six flap configurations at the cruise flight condition. 
Table 6-3: Cruise contours of Mach and required $\alpha$

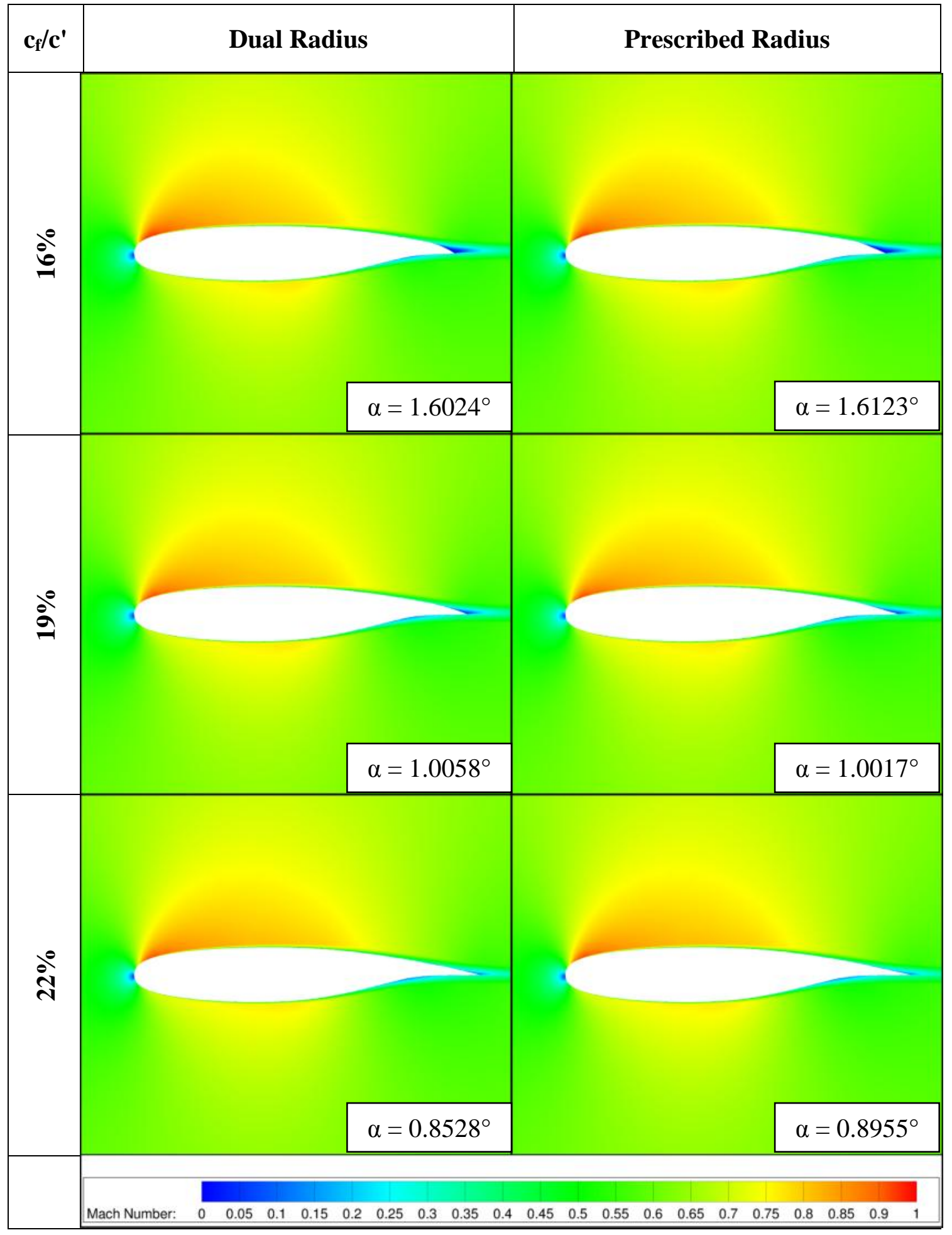


Starting at the leading edge on the upper surface of each airfoil, the characteristics of the supercritical airfoil are evident with a considerable expansion caused by the large leading edge radius seen by the sudden increase in Mach number. The first trend between the different flaps that can be observed is the variation in magnitude of the initial acceleration of the flow on the upper surface, with the shorter flaps encountering greater velocities on the forward region of the upper airfoil surface. These faster velocities of the shorter flaps are products of the slightly larger $\alpha$ necessary to achieve the target cruise $C_{1}$. As the flap length of each configuration lengthens, the needed $\alpha$ and resulting velocity in this area notably decreases. Aft of the initial acceleration of flow on the upper surface, the flow stays at a somewhat constant velocity due to the transonic airfoil's low surface curvature in this section. The upper surface shock is around the same location on each airfoil, denoted by the gradient $\sim 58 \%$ chord, showing deceleration of the flow. From the location of the shock and aft, the difference in flap shape can be seen mostly by the wake each airfoil leaves. The shorter flaps with greater $\theta$ and $\alpha$, have a more pronounced area of low speed flow. From the observations presented above, the increased upper surface velocities and low airspeed wake of the shorter flap configurations will lead to increased cruise drag.

\section{Drag and Moment Data}

All resulting performance data was taken from the $\alpha$ that each airfoil/flap configuration required (as labeled above in Table 6-3) to achieve the target cruise $\mathrm{C}_{1}$. To visualize the required $\alpha$ for each flap configuration given the flap's length see Figure 6-59 below. The analytical data of $\mathrm{C}_{\mathrm{d}}$ vs. flap length is also presented below in Figure 6-60. 


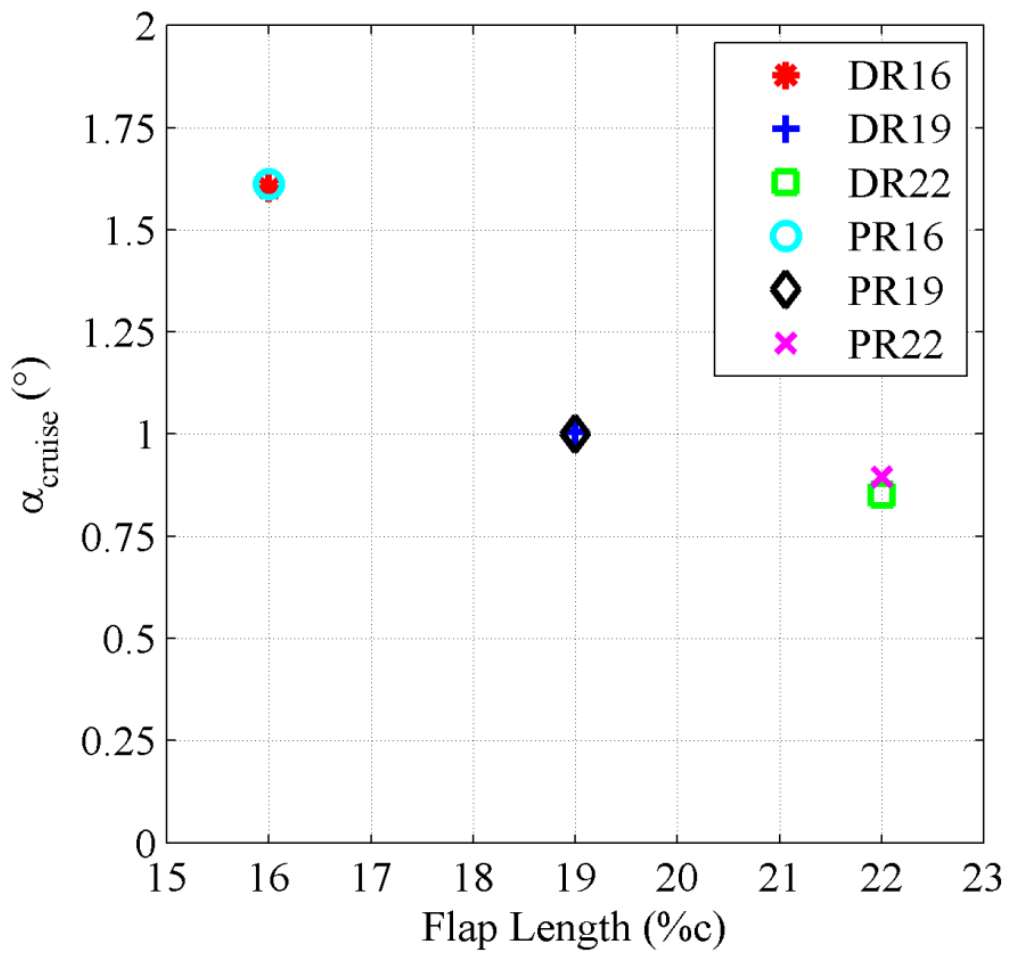

Figure 6-59: Required $\alpha$ for cruise given flap length for $0^{\circ}$ flap configurations

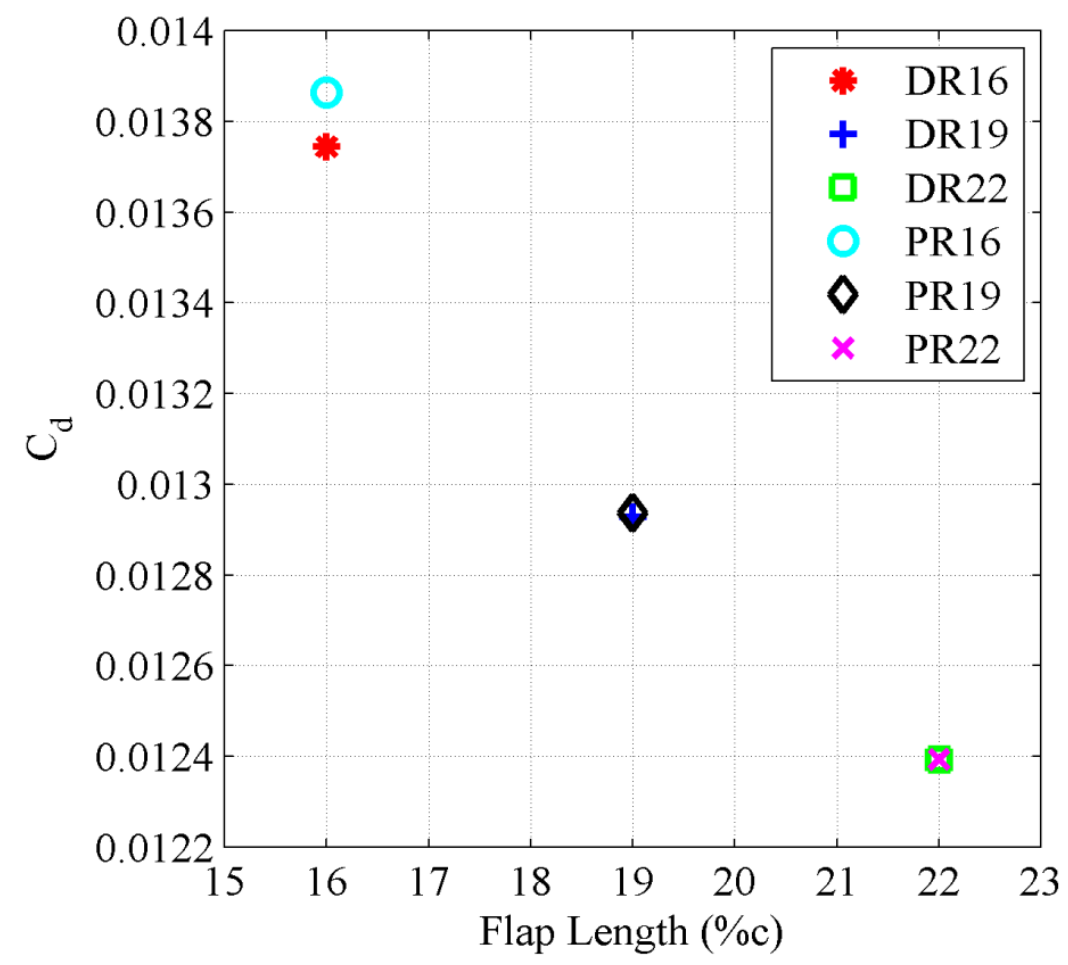

Figure 6-60: Drag given flap length for $0^{\circ}$ flap configurations 
The required cruise $\alpha$ displayed an inverse logarithmic relation with flap length on Figure 6-59, getting significantly larger as the flap length shortened. The $\alpha$ values dropped an average of $38 \%$ between the $16 \%$ and $19 \%$ flaps, and lowered to an average difference of $13 \%$ between the $19 \%$ and $22 \%$ flaps. Both $16 \%$ and $22 \%$ flap lengths show that the PR flaps needed slightly more $\alpha$ to maintain cruise lift, which could show the effect of the $\theta$ value at each flap's trailing edge. From Figure 6-60, the drag follows a similar trend to that of $\alpha$ with the shorter flaps experiencing more drag than the longer flaps. Data points to a direct relation between $\alpha$ and $\mathrm{C}_{\mathrm{d}}$, with the shorter flaps, having more $\alpha$, performing notably worse than the longer flaps. The drag values dropped an average of $6.3 \%$ from the $16 \%$ to the $19 \%$ flaps, which kept decreasing with an average drop of $4.2 \%$ between the $19 \%$ and $22 \%$ flaps. The $19 \%$ and $22 \%$ flaps showed little to no difference in drag between configurations, where the $16 \%$ prescribed radius flap had slightly more drag $(\sim 1 \%)$ than the corresponding dual radius flap. To further investigate the $\alpha$ and drag relationship as well as the resulting L/D values of each configuration, Figure 6-61 and Figure 6-62 were generated (below). 


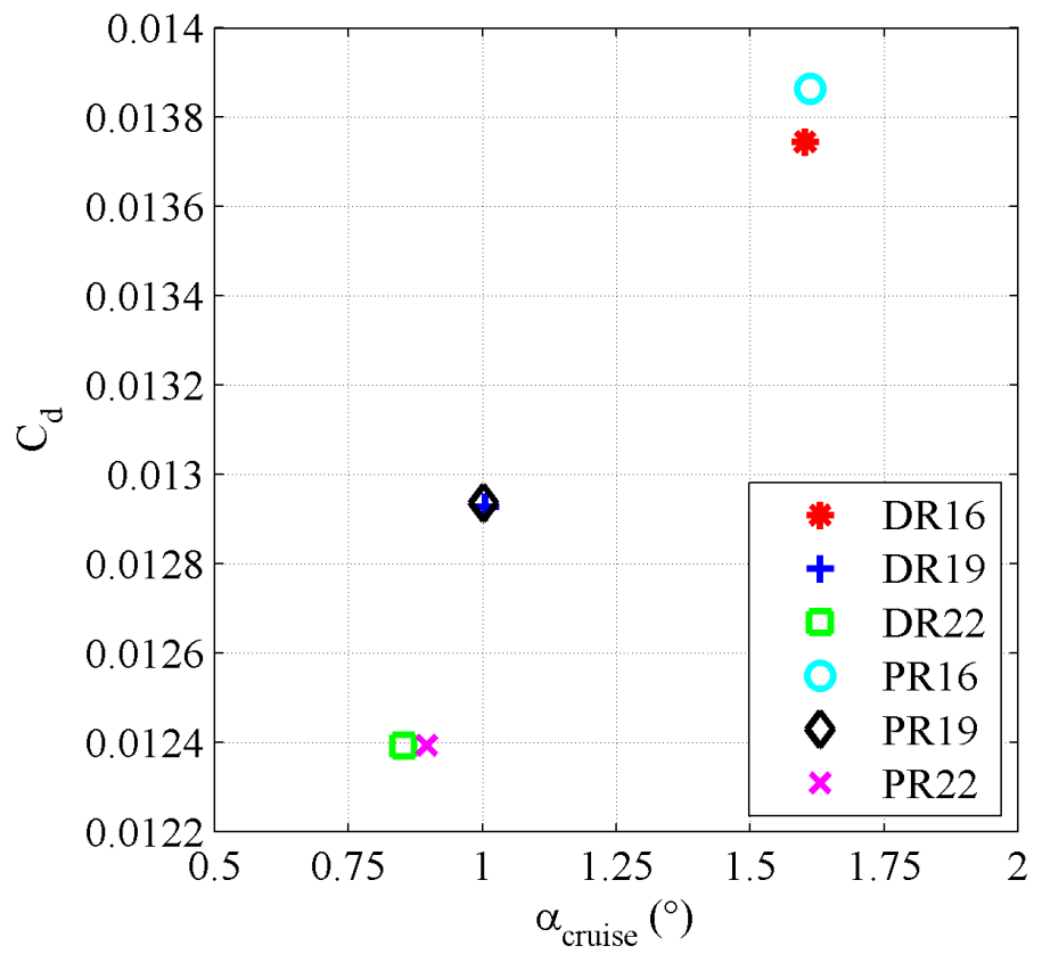

Figure 6-61: Drag and cruise $\alpha$ for $0^{\circ}$ flap configurations

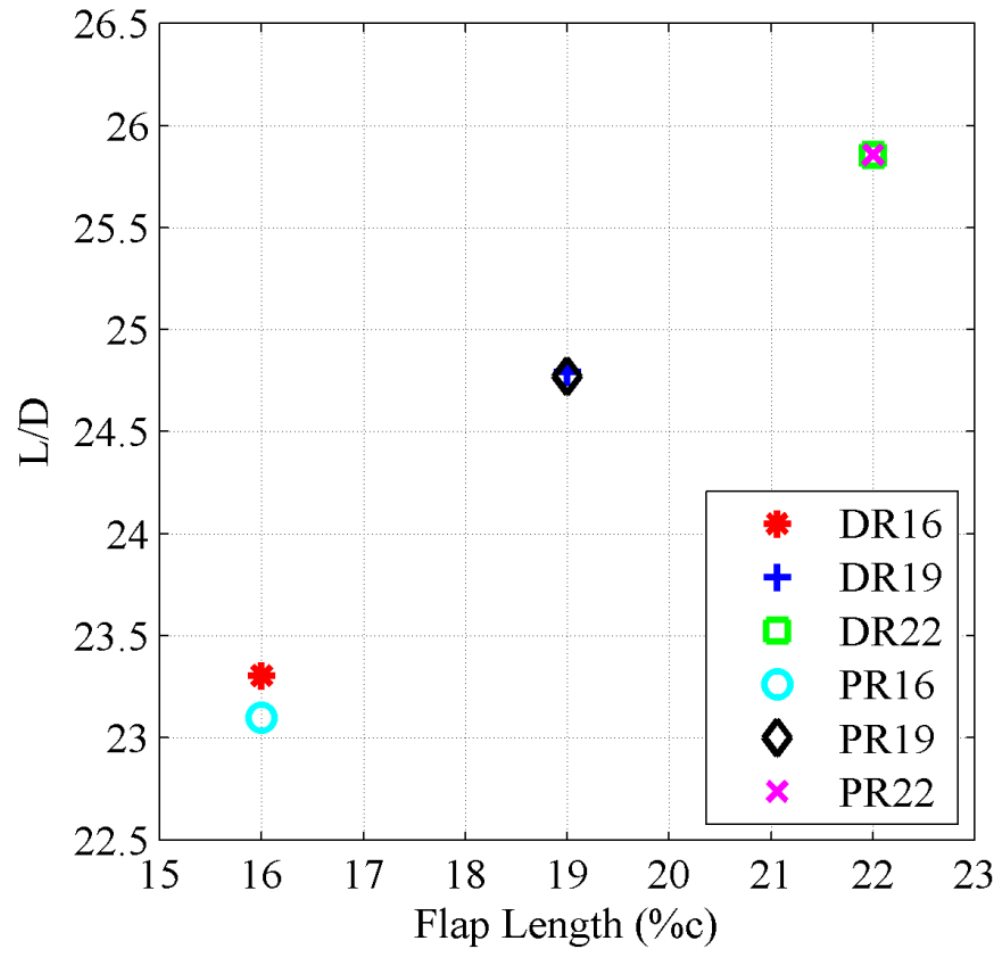

Figure 6-62: Lift-to-drag ratios for $0^{\circ}$ flap configurations 
The $\mathrm{C}_{\mathrm{d}}$ vs. $\alpha$ plot for the different flap configurations again shows the significant effect that $\alpha$ has on the drag of each airfoil and flap, demonstrating a logarithmic relationship. The overall trend can be taken as the flap length increases and required $\alpha$ decreases, the drag also decreases. Figure 6-62 shows all the flap configurations reaching L/D values between 23 and 25.9, with the longer flaps achieving the largest values. Due to the lift of all the flap configurations being the same value, the L/D trends represent the exact inverse of what was observed in the $\mathrm{C}_{\mathrm{d}}$ vs. flap length plot, Figure 6-60.

To examine the cruise trim requirements of each of the flap configurations, airfoil quarter-chord moment data was presented below in the plot of $\mathrm{C}_{\mathrm{m} \text {, (c/4) }}$ vs. flap length (Figure 6-63).

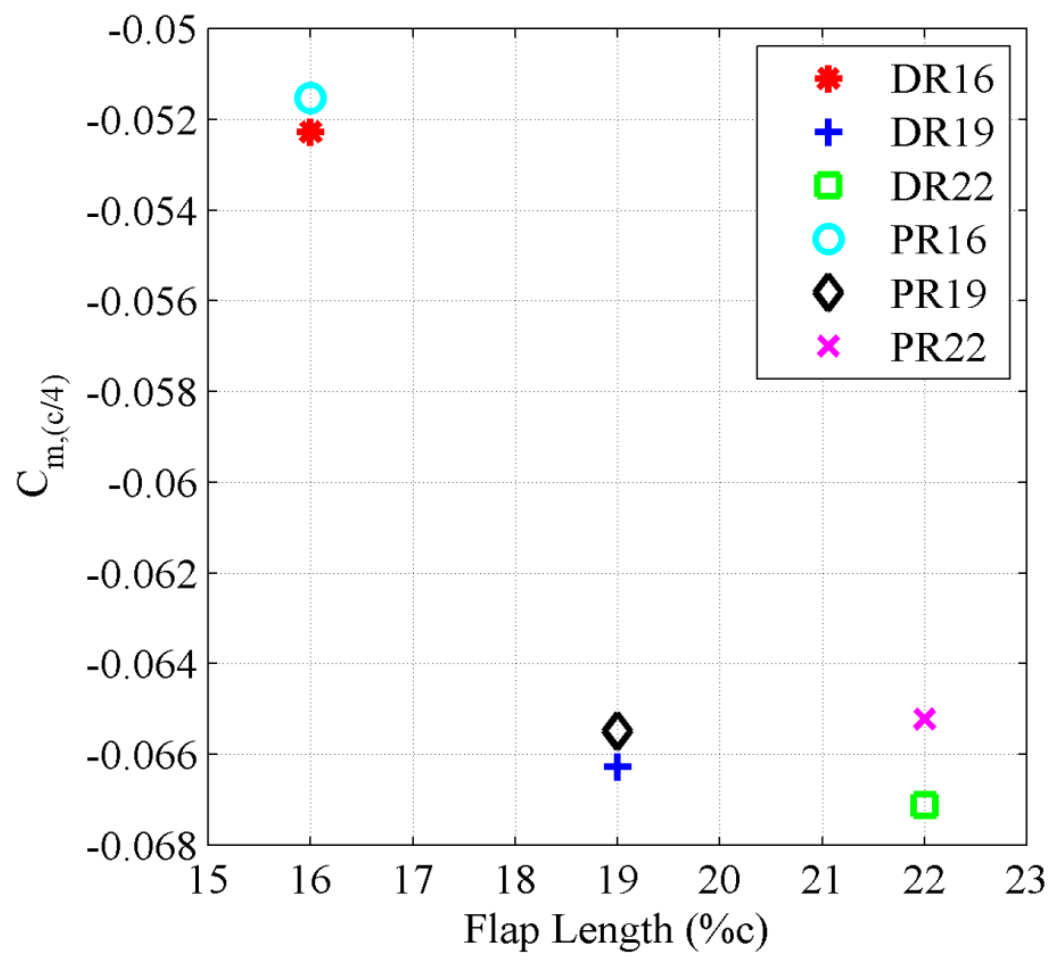

Figure 6-63: Pitching moment given flap length for $0^{\circ}$ flap configurations 
The negative pitching moments experienced by all of the airfoil/flap configurations at the target cruise conditions confirm the trim penalties that are characteristic of supercritical airfoils. $^{76}$ The data shows that the $16 \%$ flaps have significantly lower negative pitching moment than both the $19 \%$ and $22 \%$ flaps, averaging a $27.2 \%$ reduction. When comparing this data to the Mach contour table above (Table 6-3), it appears that the low speed flow occurring on the upper surfaces of the $16 \%$ flaps prior to the trailing edge reduces the negative pitching moment. When comparing the moment data of dual and prescribed radius configurations, all of the prescribed radius configurations experience reduced moments with the most significant being between the two $22 \%$ flaps of a $2.8 \%$ reduction. It is also interesting to note that the $22 \%$ prescribed radius flap achieves even less negative pitching moment than the $19 \%$ prescribed radius flap. This data points to the configurations, of the same length, carrying the least $\theta$ and radius of curvature off the trailing edge to have the most reduced negative pitching moments. The moment data also suggests that the low airspeed flow encountered with the shorter flaps reduced the amount of negative moment.

\section{Pressure and Skin Friction Data}

The pressure and skin friction distributions of each flap configuration were analyzed at the simulated cruise flight conditions to observe the influence of each flap's geometry. Since the main emphasis of this study was to investigate the different distributions on each of the flap surfaces, only the aft region of each airfoil is presented in this section. Although it should be noted, both the pressure and skin friction distributions of all the configurations displayed small oscillations on the upper and lower surface of the forward $3 / 4$ of the airfoil. All of the $\mathrm{C}_{\mathrm{p}}$ data displayed the common characteristics of 
supercritical airfoils, with the first being the large suction peak near the leading edge caused by the significant acceleration of flow around the large leading edge radius of the chosen super critical airfoil. From the suction peak, the pressures then slowly increased until the location of the shock, located approximately at an $\mathrm{x} / \mathrm{c}^{\prime}$ of 0.58 for all of the flap configurations. From the shock location and aft, the flow decelerated more rapidly (shown by increasing pressure) until the beginning of each flap surface.

The $\mathrm{C}_{\mathrm{p}}$ distribution of the trailing edge/flap surface for all the configurations is presented below in Figure 6-64.

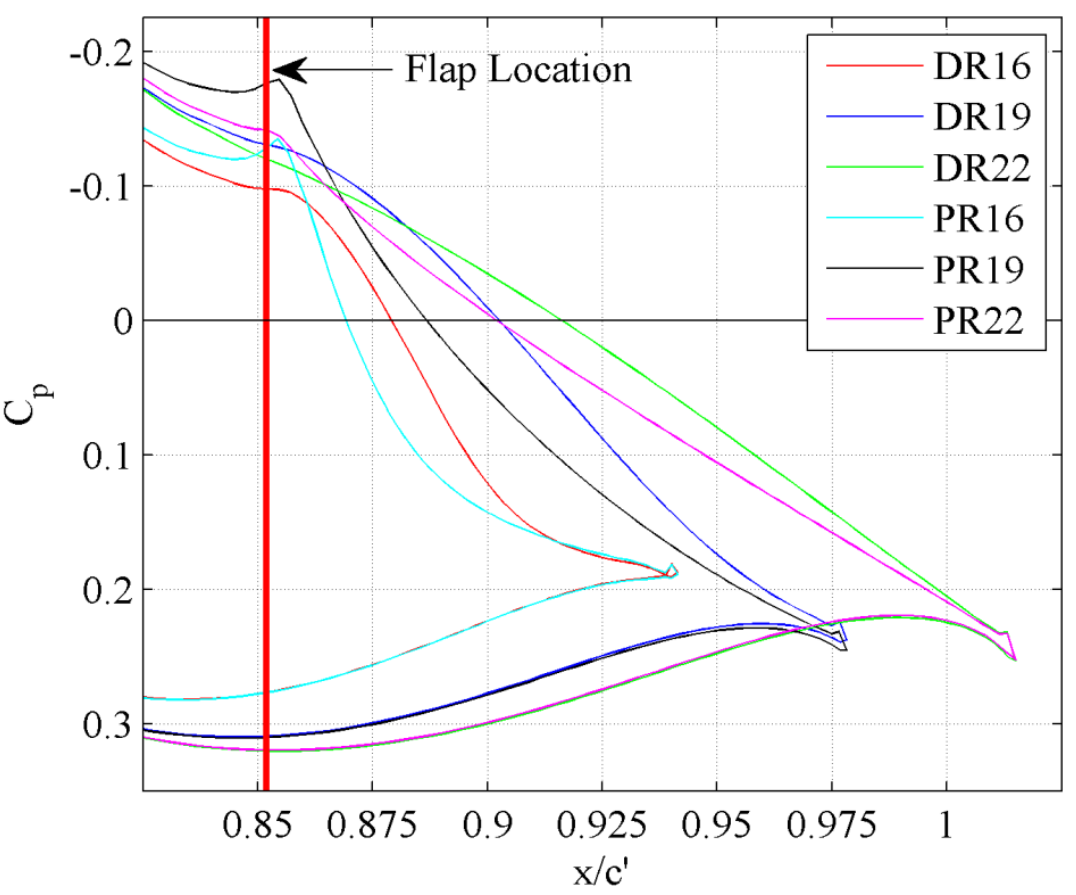

Figure 6-64: Trailing edge/flap surface pressure distribution, cruise $\alpha, 0^{\circ}$ flaps

Similar to the $60^{\circ}$ and $90^{\circ}$ flap deflections, all of the dual radius flap configurations carried lower pressures over their upper flap surfaces showing faster moving flow. All prescribed radius flaps experienced a sharp pressure decrease at the beginning of the flap then steadily increased over the beginning of the flap surface. All 
of the corresponding dual radius flaps experienced a much more gradual pressure fluctuation at the start of the flap surface while maintaining lower pressures of the entirety of the flap. The cause of these pressure fluctuations in the prescribed radius flaps is a result of the significant surface curvature changes that the prescribed radius flaps experience in this region. Note that due to the $0^{\circ}$ flap deflection only the secondary surface of each flap is exposed, meaning only $r_{2}$ is exposed for the dual radius and the cubically varying radius for the prescribed radius. At the change in surface from the original airfoil to the each of the flaps second surface, the dual radius flaps transition from the low curvature of the super critical airfoil to the constant curvature values of the $\mathrm{r}_{2}$ radius. The prescribed radius flaps transition from the low curvature of the airfoil to the significantly higher curvature of $r_{1}$ then quickly reduce to zero over the length of each flap. These changes in surface curvature are displayed in Figure 6-65, below. This substantial change in the flap surface curvature at the beginning of each flap causes the acceleration/deceleration of airflow seen by the spikes in $C_{p}$ on each of the prescribed radius flaps. These spikes in pressure could lead to separation. The longer prescribed radius flaps lessen this pressure fluctuation by going through the shifting in curvature in a very short distance, holding a more constant curvature over the entire flap surface. In the case of the $22 \%$ prescribed radius flap, the fluctuation is almost totally eliminated. 


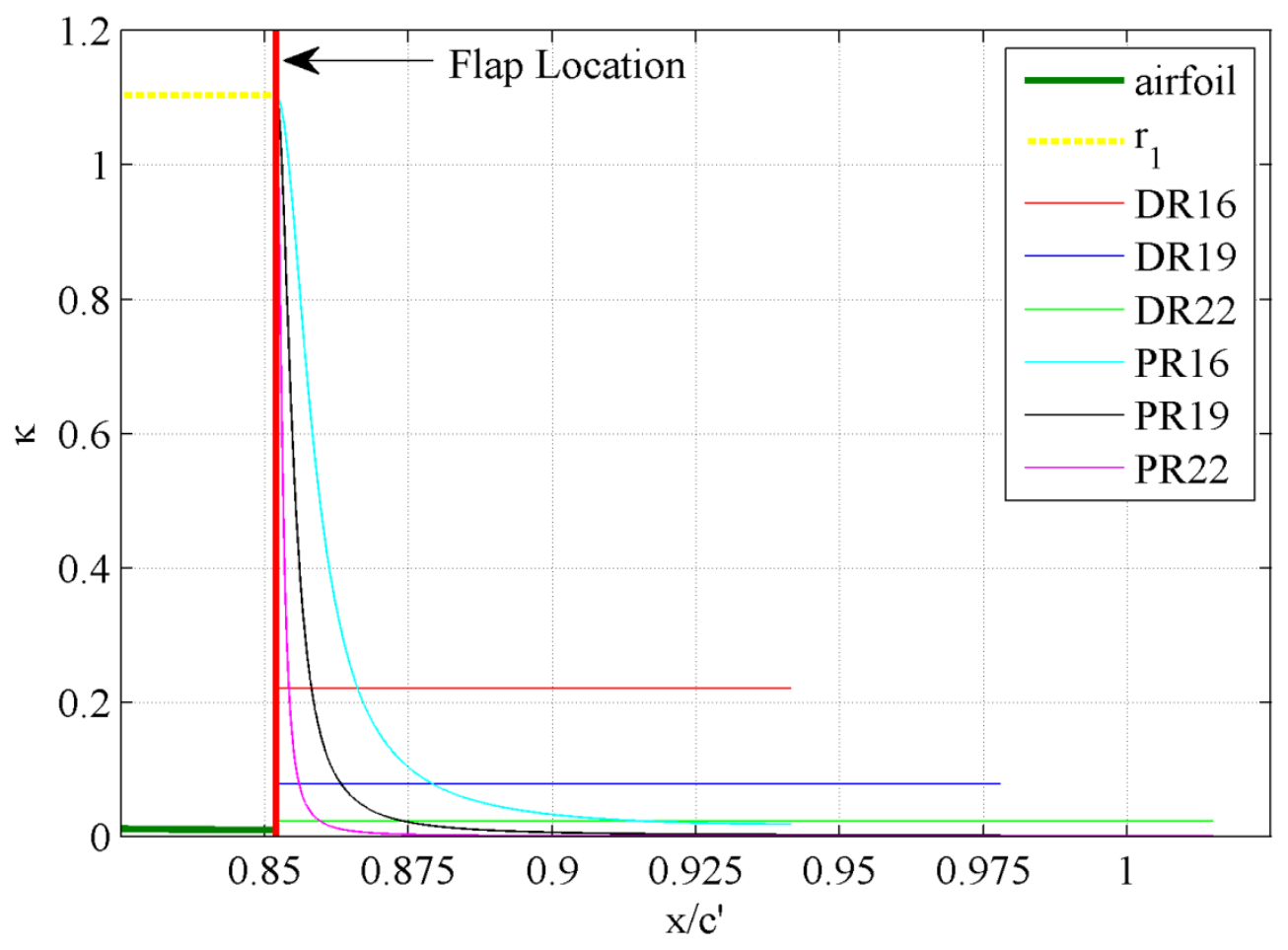

Figure 6-65: Airfoil trailing edge/flap surface curvature, cruise $\alpha, 0^{\circ}$ flaps

The skin friction coefficient distributions of the different flap configurations featured similar trends to those of the $\mathrm{C}_{\mathrm{p}}$ data with a leading spike, slowly decreasing along the upper surface until the shock location ( $58 \%)$, and then dropping off at a slightly greater rate with the deceleration of the post-shock flow. The constant decrease in skin friction continued until the beginning of the flap surface, where each configuration exhibited a different behavior. To analyze the difference in each flaps' cruise characteristics, Figure 6-66 below details the skin friction distributions on the various flap surfaces. 


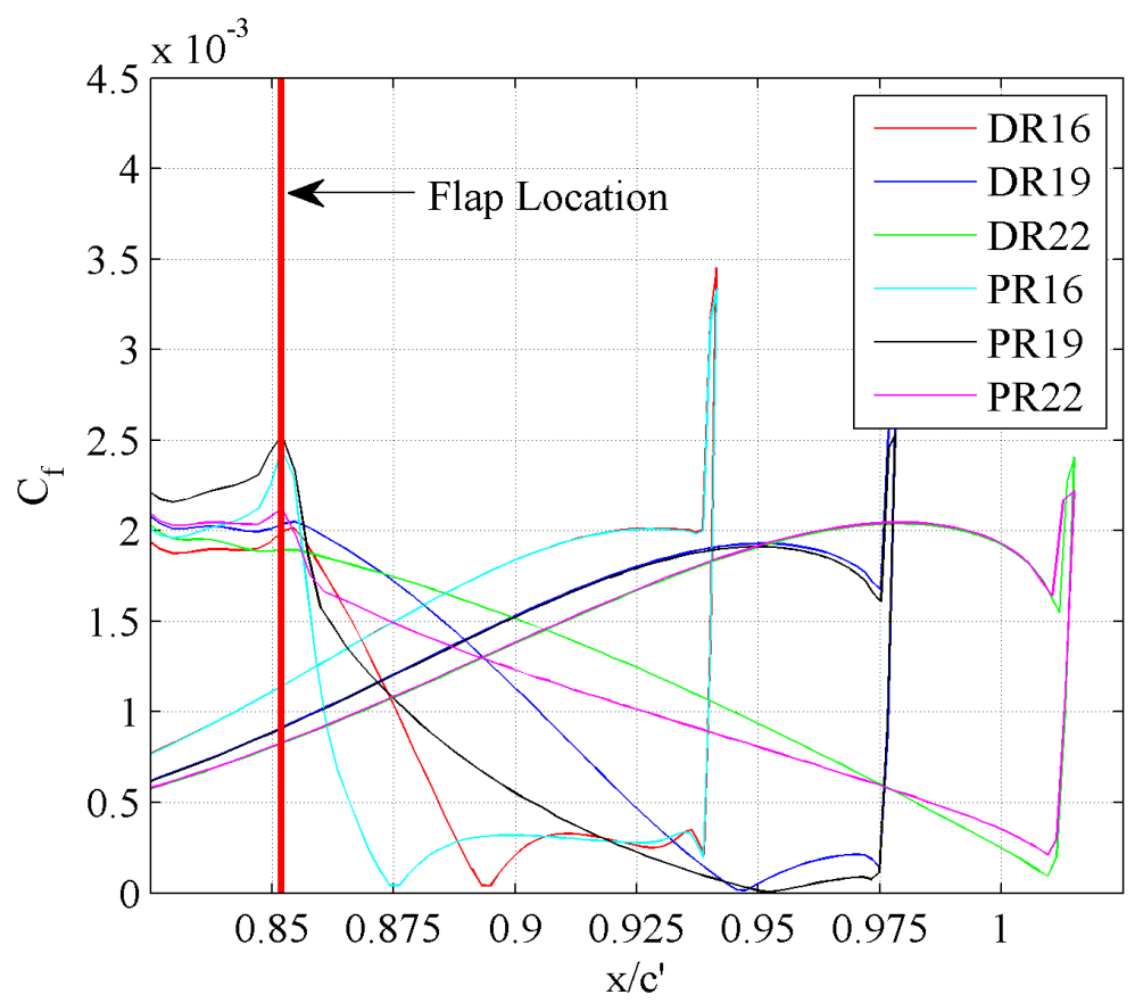

Figure 6-66: Flap surface skin friction distributions for cruise $\alpha, 0^{\circ}$ flaps

The severity of the $\mathrm{C}_{\mathrm{f}}$ drop-off on the upper surface of each flap configuration is largely a function of the length of the flap with the shorter flaps seeing the more extreme drop-offs. Both the $16 \%$ and $19 \%$ flaps saw skin friction values approach zero before the trailing edge, displaying where the low airspeed airflow occurs on the upper surface of these flaps. These locations are confirmed by the blue regions of very slow moving flow on the Mach contour plots above (Table 6-3). The skin friction of the $22 \%$ flaps did not reach zero, showing how the longest flap configurations were the best at reducing low airspeed at cruise conditions. Comparing the dual and prescribed radius flaps, the prescribed radius flaps experience the decrease in skin friction at a greater rate with the dual radius flaps maintaining higher skin friction or flow velocity over their flap surfaces due to the greater curvature over the entirety of the flap. The spike in skin friction of the 
prescribed radius configurations at the start of the flap surfaces are consistent with those of the $\mathrm{Cp}$ data, and again shows the effects of the drastically changing curvature on the local airflow. Note as the prescribed radius flaps lengthened, the skin friction fluctuations notably decrease.

The wall $\mathrm{y}^{+}$values of all of the cruise configuration simulations are presented below in Figure 6-67.

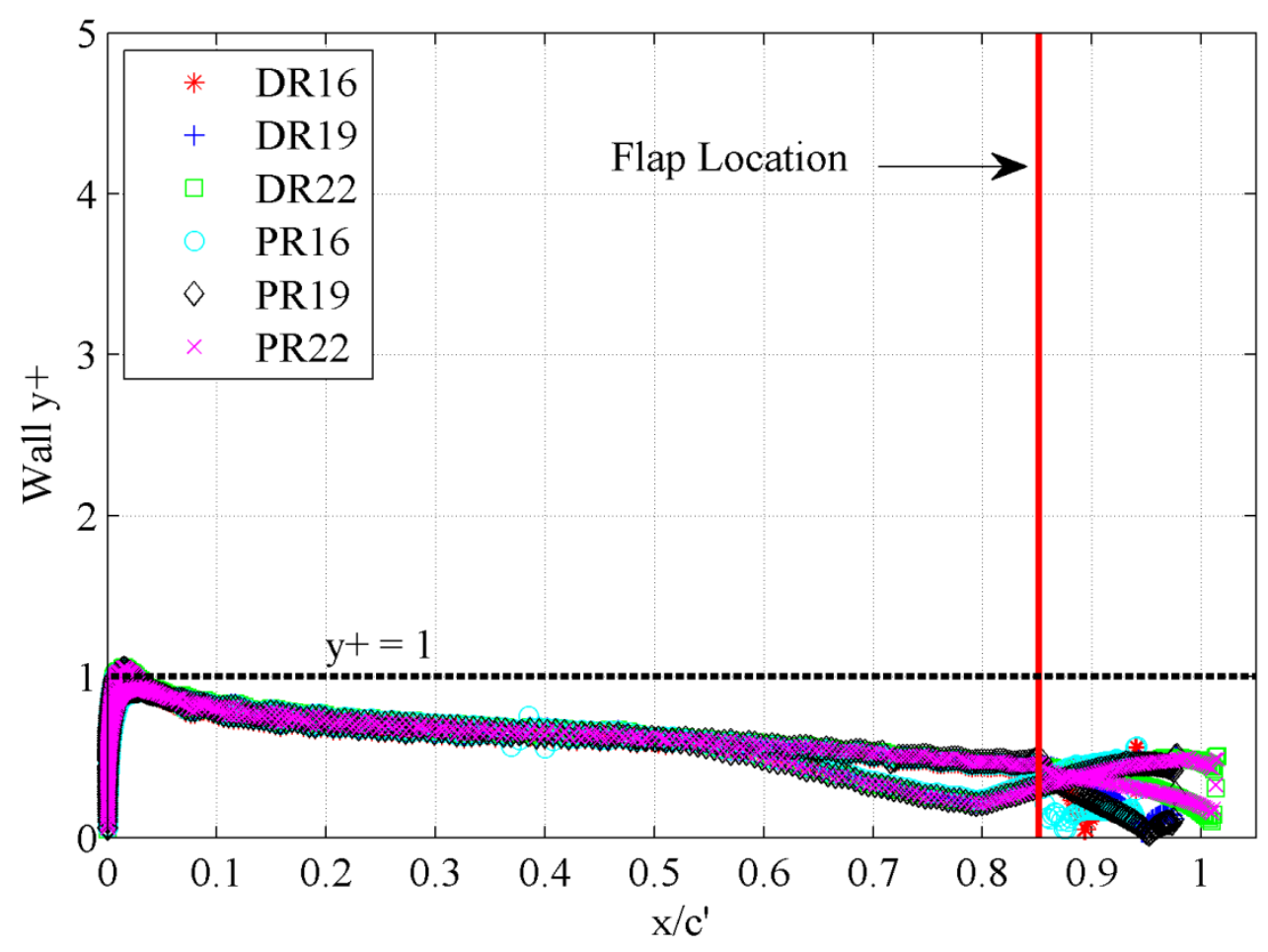

Figure 6-67: $y^{+}$values of the airfoil/flap configurations, cruise $\alpha, 0^{\circ}$ flaps

All of the cruise condition $\mathrm{y}^{+}$values stay near a value of one or below, with the largest values seen at the leading edge where the flow is accelerating around the large leading edge radius of the supercritical airfoil. The rest of the airfoil experiences values well below one, which allowed for accurate simulation of the flow with the chosen solving methods. 


\section{Flap Parameter Trends}

To examine the influence of the flap parameters on cruise performance $\left(0^{\circ}\right.$ flap deflection), the performance parameters of $\mathrm{L} / \mathrm{D}$, required cruise $\alpha$, and $\mathrm{C}_{\mathrm{m}}$, (c/4) were plotted against each flap's geometric parameters. These performance parameters were studied to evaluate each flap configuration's lifting efficiency during cruise as well as their resulting pitching moments that require elevator deflection to counter during cruise (trim drag). The main goal of the cruise setting of each flap/airfoil was to maximize L/D

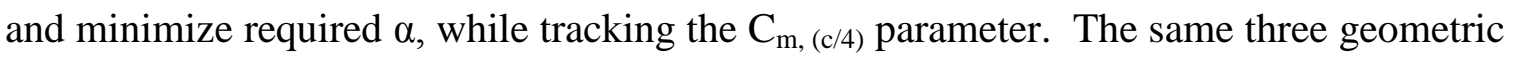
parameters were investigated, the flap's departure angle $(\theta)$, the flap chord-to-airfoil chord ratio $\left(\mathrm{c}_{\mathrm{f}} / \mathrm{c}^{\prime}\right)$, and the radius ratio $\left(\mathrm{r}_{2} / \mathrm{r}_{1}\right.$ or $\left.\mathrm{r}_{2 \_a v g} / \mathrm{r}_{1}\right)$.

Figure 6-68, Figure 6-69, and Figure 6-70 below display the cruise performance trends with variation of the flap's departure angle $(\theta)$. Note that each flap/airfoil was analyzed at their individual required $\alpha$ to obtain the 2-D design cruise $C_{1}$ of 0.320 . 


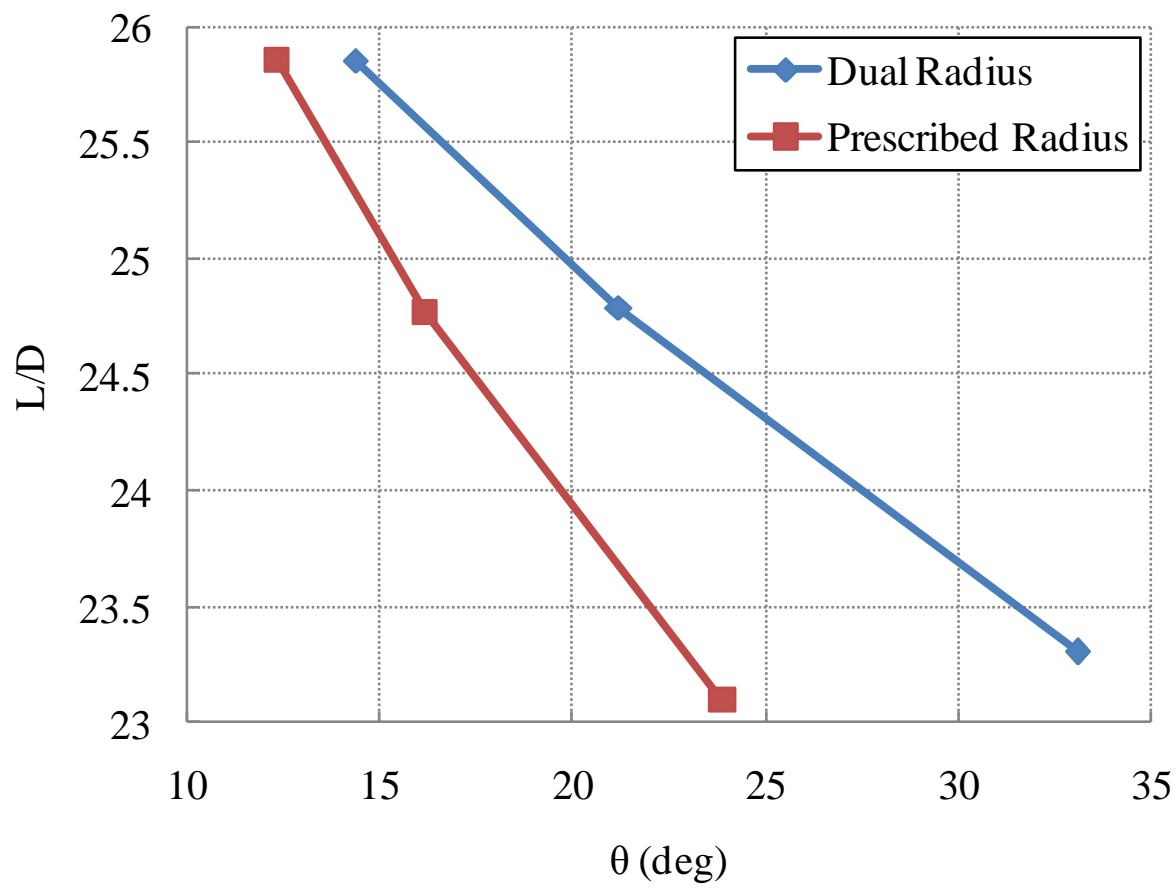

Figure 6-68: Lift-to-drag ratios for flap configurations given $\theta$

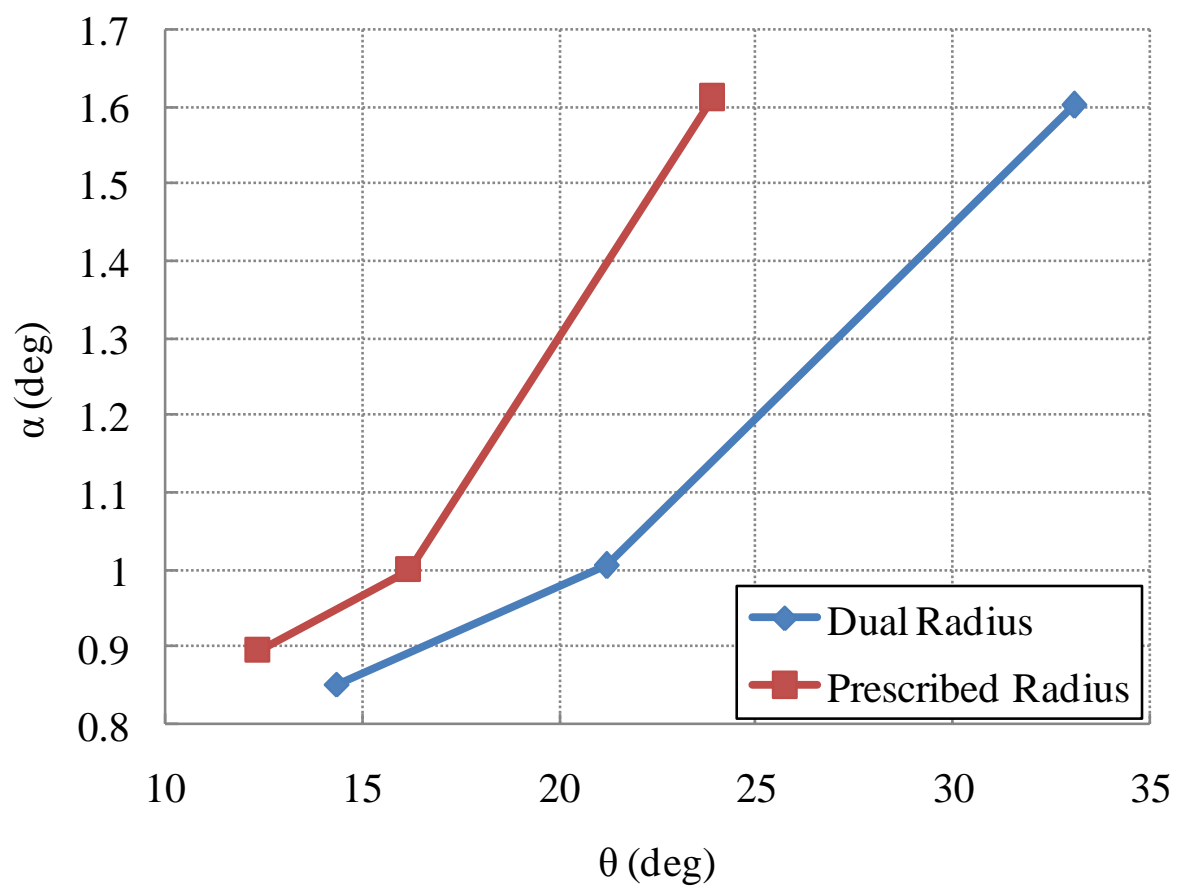

Figure 6-69: Required cruise $\alpha$ for flap configurations given $\theta$ 


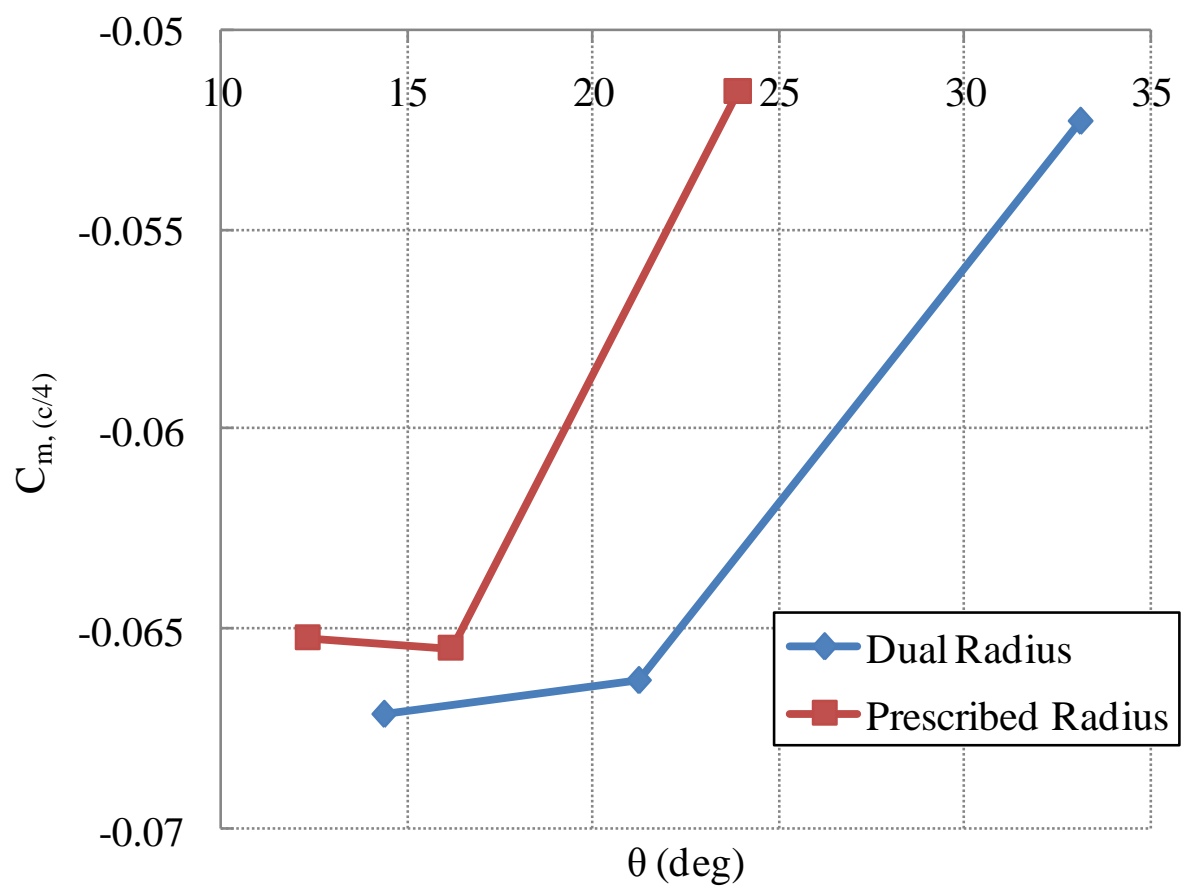

Figure 6-70: Pitching moment for flap configurations given $\theta$

The above plots display the very similar performance of the dual radius and prescribed radius flaps over their range of $\theta$ values. They both achieve the same L/D (25.86) at their lowest $\theta$ values, $14.36^{\circ}$ for the dual radius and $12.35^{\circ}$ for the prescribed radius. The prescribed radius $L / D$ trend followed that of the dual radius but translated to the lower $\theta$ values.

The required $\alpha$ results, presented in Figure 6-69 above, showed how the dual radius configurations required less cruise $\alpha$ given the flaps' trailing edge $\theta$ than the prescribed radius flaps. This shows that even with the same $\theta$, the prescribed radius flaps would need slightly more $\alpha$ to obtain the same amount of lift generated by the dual radius configurations that carry more curvature through the entirety of each flap upper surface. At each flaps' higher $\theta$ values, where low airspeed flow is present on the airfoil's trailing 
edge (Table 6-3), they require very similar $\alpha$ 's. Though at the lower $\theta$ values, the dual radius flap required approximately $0.05^{\circ}$ less $\alpha$.

The $\mathrm{C}_{\mathrm{m},(\mathrm{c} / 4)}$ data displays a notable moment drop-off at each flaps larger $\theta$ values. This is again caused by the low speed airflow at the trailing edge for these specific configurations, where the trailing edge of each flap produces less lift. The lower two $\theta$ values of the prescribed radius maintained a lower magnitude of negative pitching moment when compared to the dual radius, with the lowest $\theta$ prescribed radius flap producing the smallest moment of -0.065 . Note that the $C_{m,(c / 4)}$ starts to lessen again as $\theta$ decreases for the prescribed radius flaps, where the dual radius flaps had yet to decrease the moment for the $\theta$ evaluated in this study.

Figure 6-71, Figure 6-72, and Figure 6-73 below depict the cruise performance trends given the flap chord-to-modified airfoil chord length ratio $\left(\mathrm{c}_{\mathrm{f}} / \mathrm{c}^{\prime}\right)$.

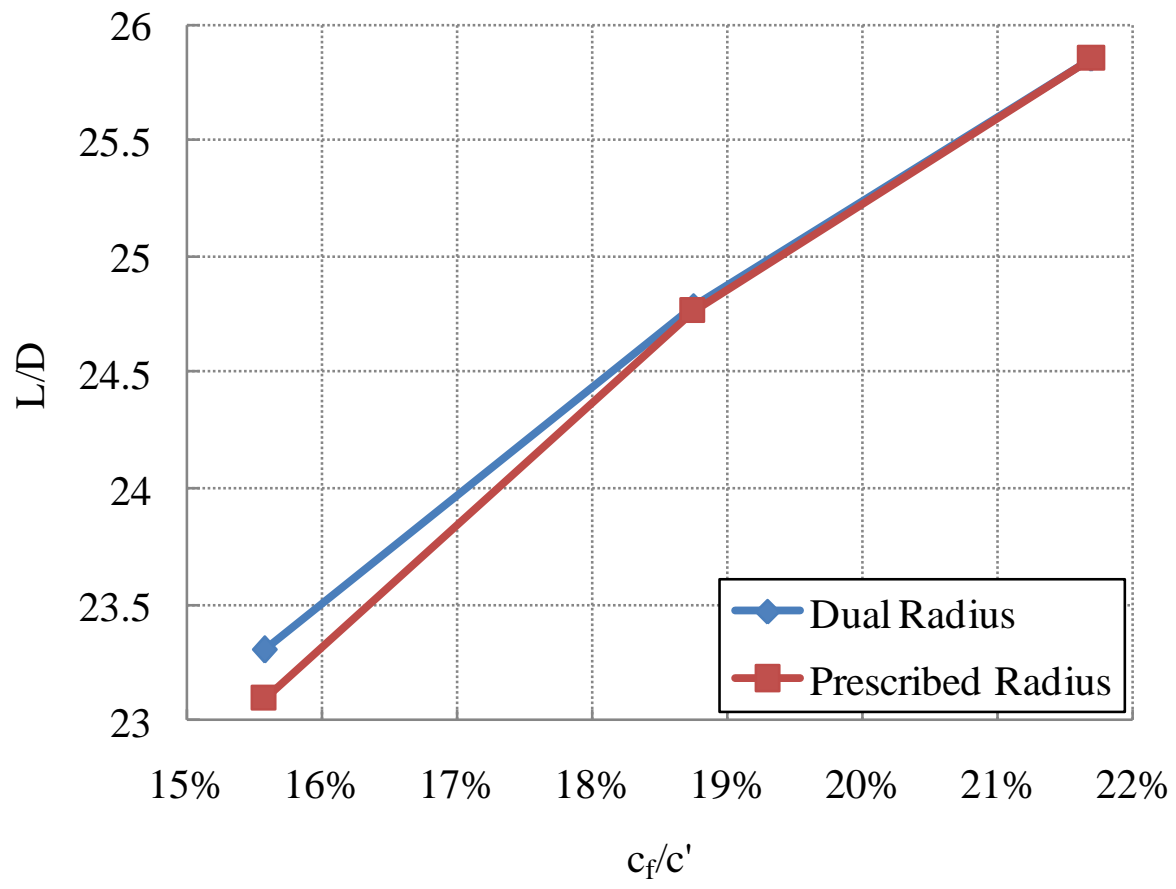

Figure 6-71: Lift-to-drag ratios for flap configurations given $c_{\mathrm{f}} / \mathrm{c}$ 


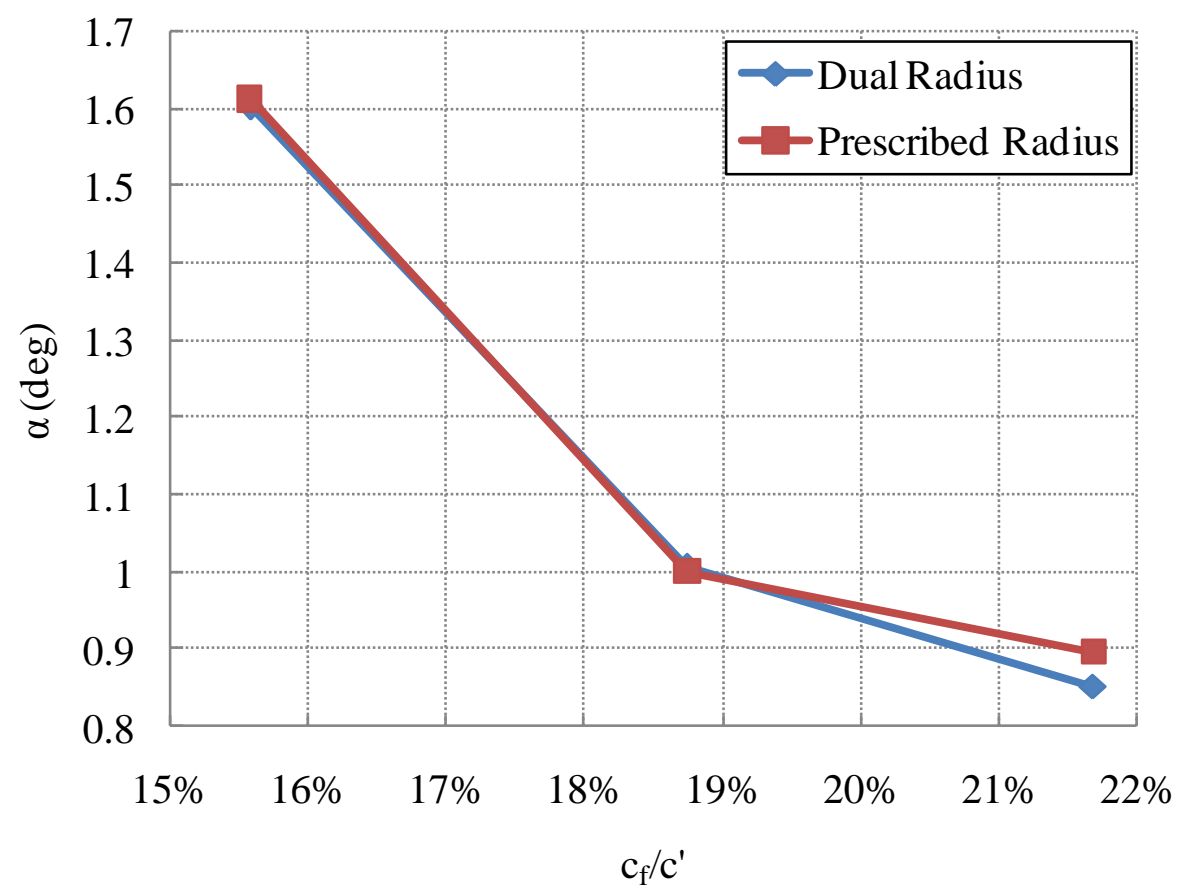

Figure 6-72: Required cruise $\alpha$ for flap configurations given $c_{f} / c^{\prime}$

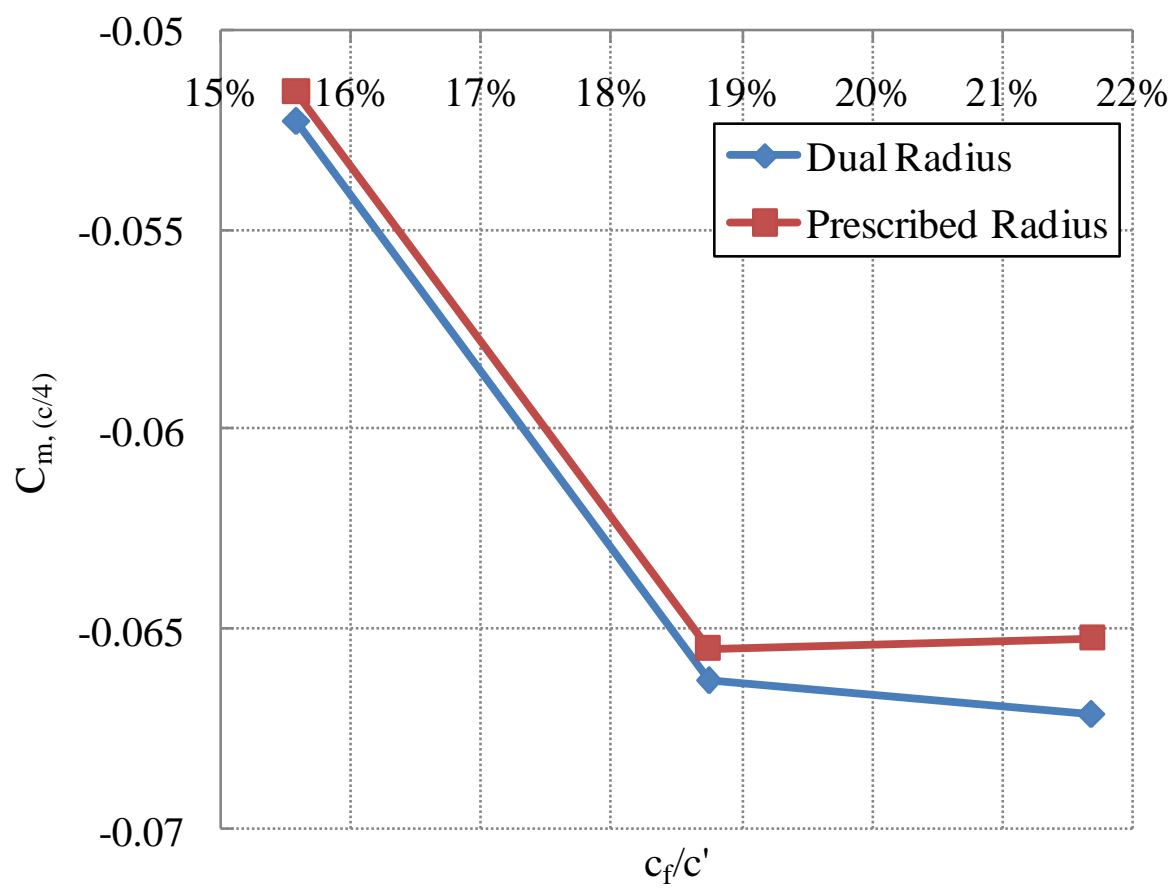

Figure 6-73: Pitching moment for flap configurations given $c_{\mathrm{f}} / \mathrm{c}^{\text {' }}$ 
Both dual radius and prescribed radius flap configurations again display very similar trends given $\mathrm{c}_{\mathrm{f}} / \mathrm{c}^{\prime}$ ratios. They both experience almost linear $\mathrm{L} / \mathrm{D}$ vs. $\mathrm{c}_{\mathrm{f}} / \mathrm{c}^{\prime}$ trends, with the dual radius slightly outperforming the prescribed radius flaps at lower $\mathrm{c}_{\mathrm{f}} / \mathrm{c}^{\prime}$ values and then both converging to the same $\mathrm{L} / \mathrm{D}$ values at longer flap lengths. The L/D trend clearly shows that longer flaps, for either configuration, perform the best for cruise conditions due to their lifting efficiency with minimal drag.

The required $\alpha$ vs. flap length trends for both flap configurations suggest a minimum at flap lengths slightly larger than those studied in this analysis. The dual radius flap reaches a slightly lower required $\alpha$ at the $22 \%$ flap length, which is a result of the increased flap surface curvature of the dual radius over the prescribed radius. The required $\alpha$ trends also demonstrate that flaps longer than $22 \%$, for either configuration, will not have as much benefit in reducing the required $\alpha$ for cruise conditions.

The pitching moment data vs. flap length again shows the significant moment reduction of the shorter flap configurations $(16 \%)$ due to the low airspeed flow present at the trailing edge. The trends for both configurations suggest a local maximum for negative pitching moment over the flap lengths examined. These flap lengths correspond to the flap lengths that provided the least required $\alpha$ from the previous trends. The negative pitching moment can be slightly reduced by increasing the flap lengths closer to $\mathrm{a} \mathrm{c}_{\mathrm{f}} / \mathrm{c}^{\prime}$ of $22 \%$, which would reduce the trim drag penalty associated with the moment. The prescribed radius flaps maintained an average of 0.001 lower in pitching moment compared to the dual radius for the intermediate (19\%) and long (22\%) flap lengths. Again, much of this negative pitching moment can be attributed to the supercritical airfoil of the design. 
The cruise performance trends for the radius ratio $\left(\mathrm{r}_{2} / \mathrm{r}_{1}\right)$ of the dual radius flaps are presented in Figure 6-74, Figure 6-75, and Figure 6-76. The prescribed radius ratio $\left(\mathrm{r}_{2 \_ \text {avg }} / \mathrm{r}_{1}\right)$ cruise performance trends are shown in Figure 6-77, Figure 6-78, and Figure 6-79. Note that the dual radius and prescribed radius configurations were plotted separately due to the difference in $r_{2} / r_{1}$ to $r_{2 \_a v g} / r_{1}$ magnitudes.

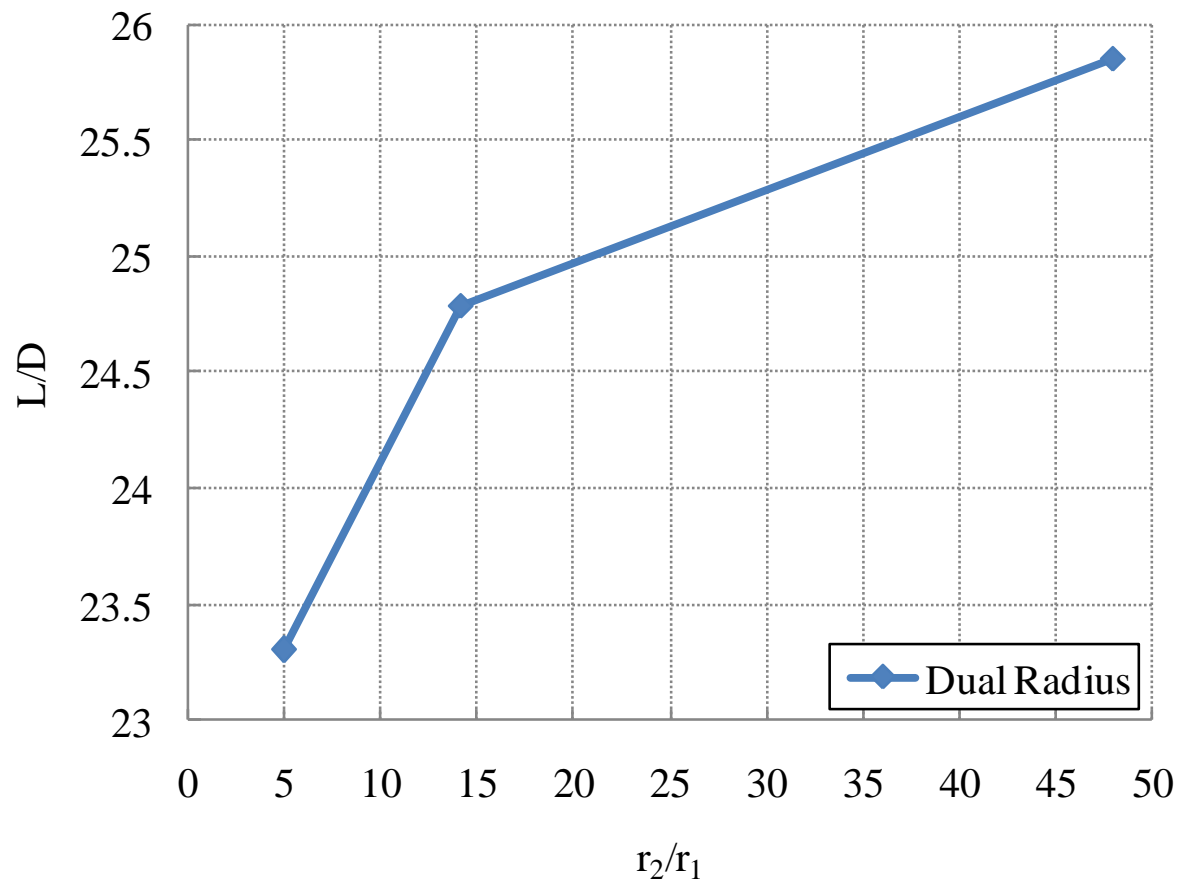

Figure 6-74: Lift-to-drag ratios for dual radius given $r_{2} / \mathbf{r}_{1}$ 


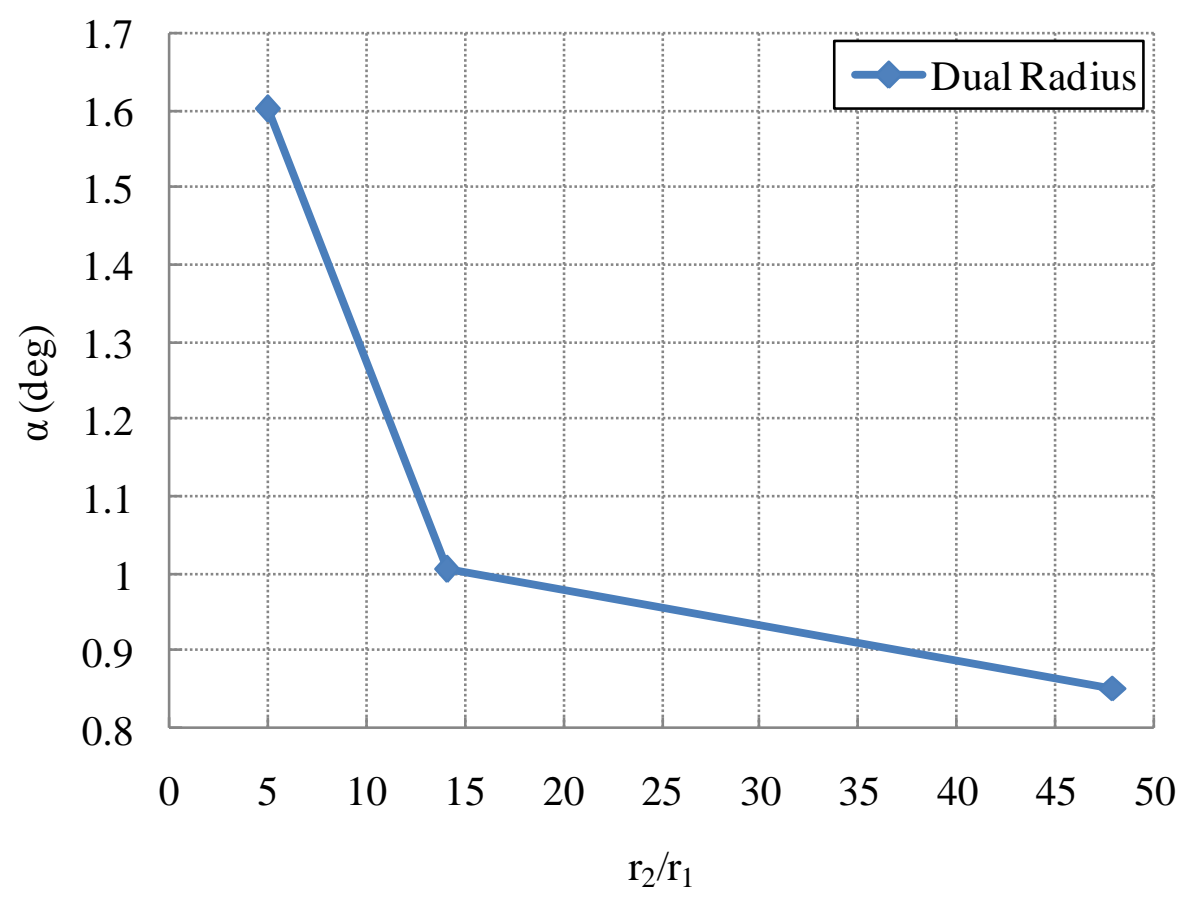

Figure 6-75: Required cruise $\alpha$ for dual radius given $r_{2} / \mathbf{r}_{1}$

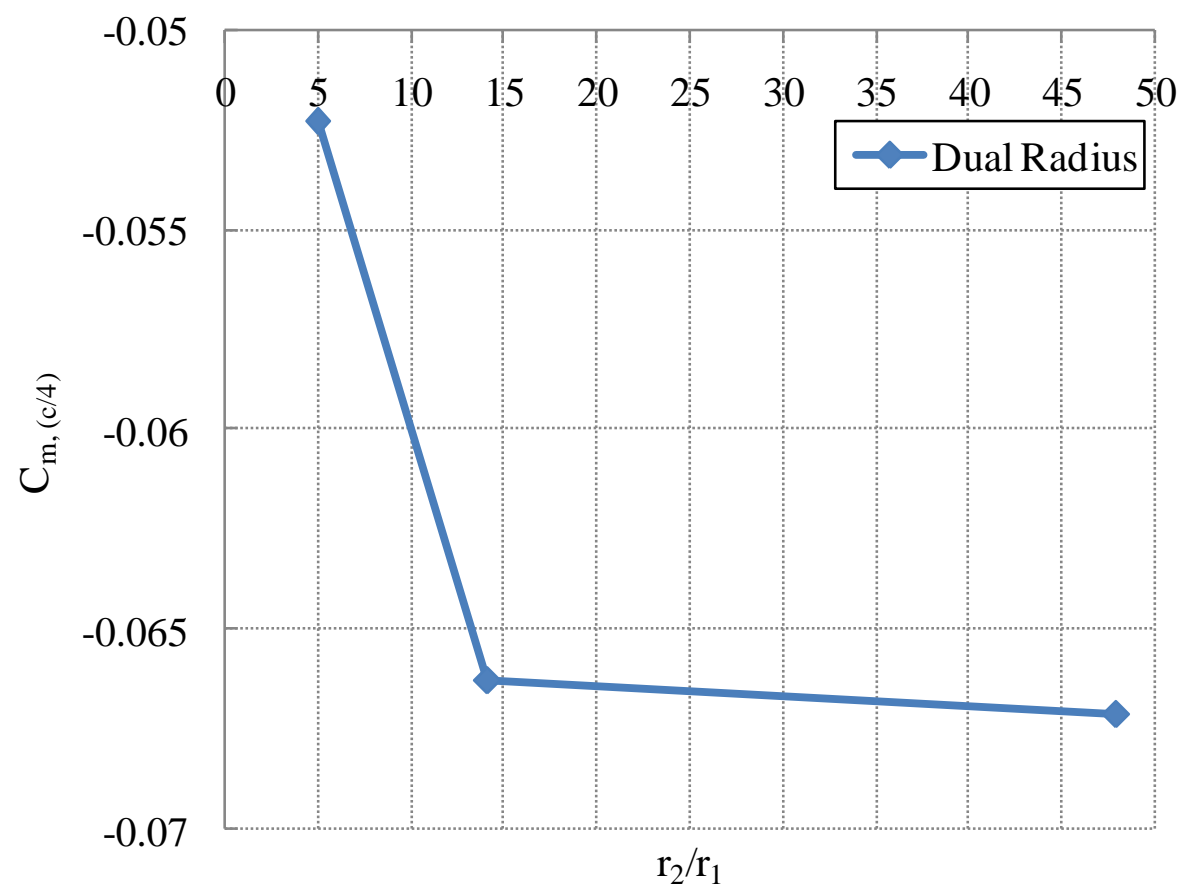

Figure 6-76: Pitching moment for dual radius given $\mathbf{r}_{2} / \mathbf{r}_{1}$ 


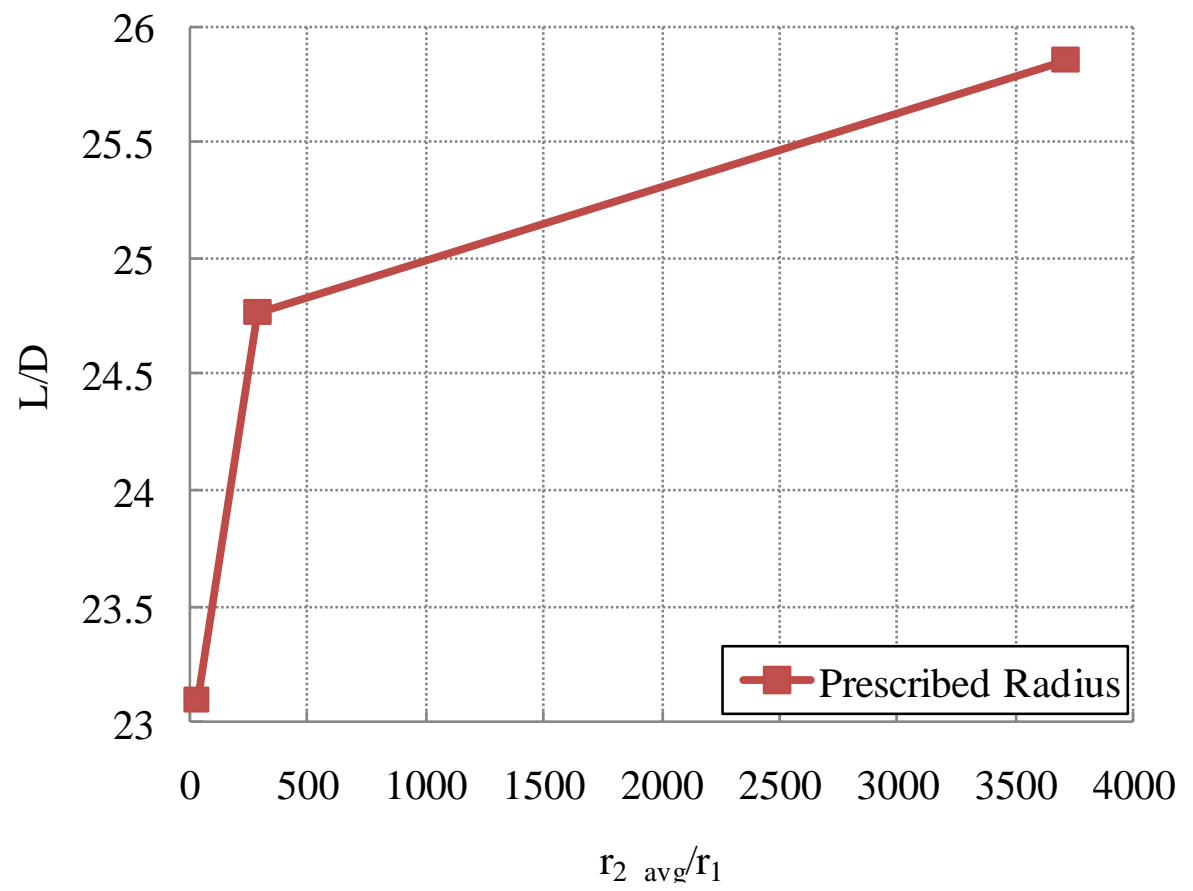

Figure 6-77: Lift-to-drag ratios for prescribed radius given $\mathbf{r}_{2 \_a v g} / \mathbf{r}_{1}$

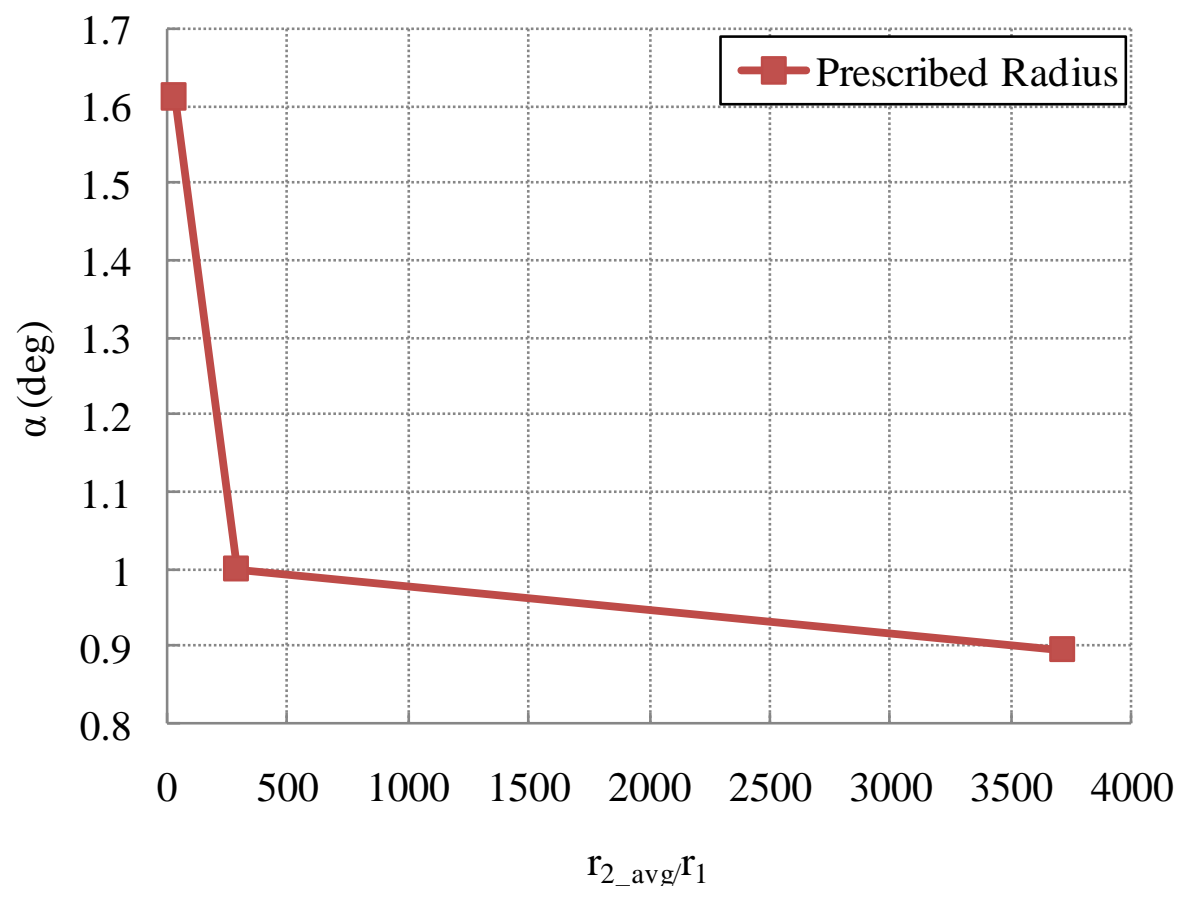

Figure 6-78: Required cruise $\alpha$ for prescribed radius given $\mathbf{r}_{2 \_ \text {avg }} / \mathbf{r}_{1}$ 


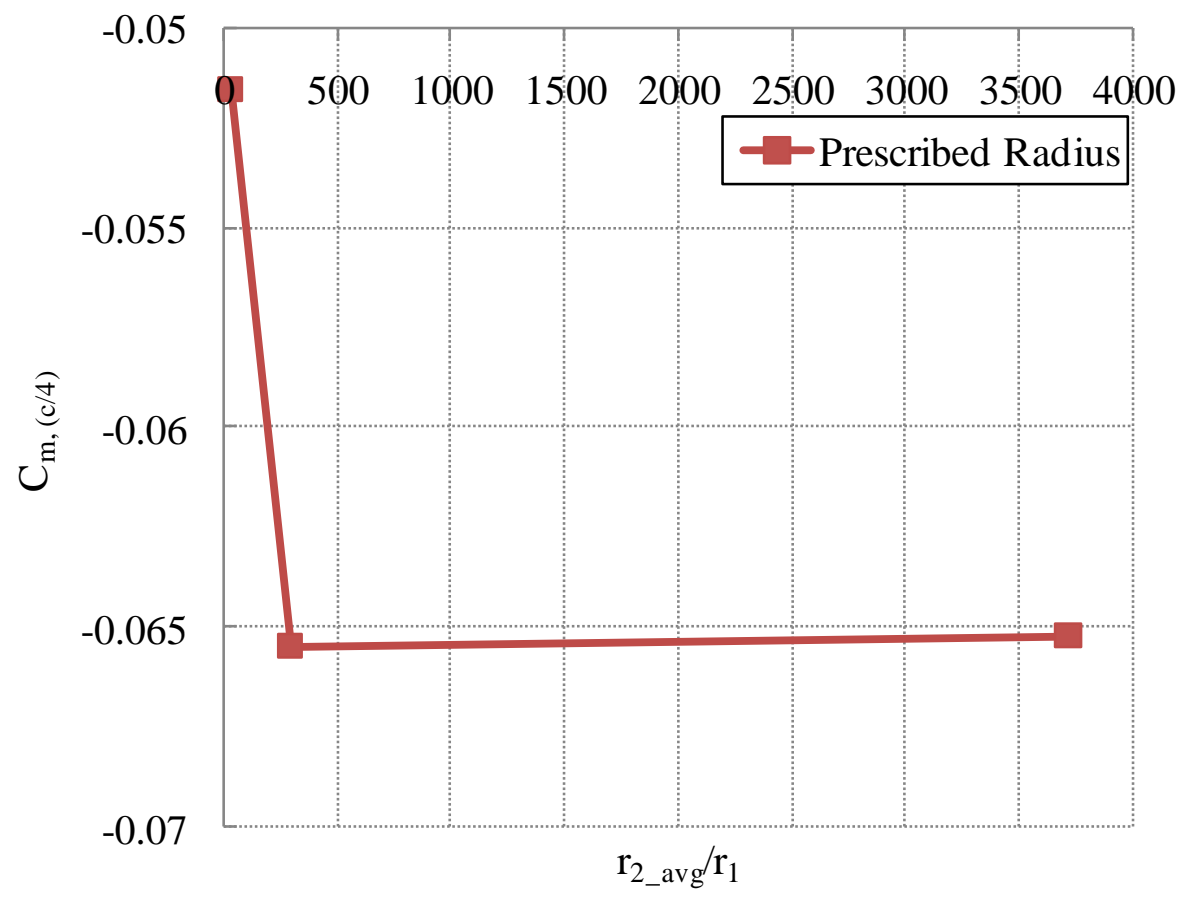

Figure 6-79: Pitching moment for prescribed radius given $\mathbf{r}_{2 \_a v g} / \mathbf{r}_{1}$

Both flap configurations exhibited similar trends for all the L/D performance parameter, with both displaying L/D data reaching a maximum at the larger radius ratios. The two configurations differentiate by the prescribed radius flap reaching a larger L/D value at much relatively lower radius ratios compared to the dual radius. This could be a result of the skewed spread of radius ratio data for the prescribed radius flaps, as seen in approach/landing and takeoff performance trends. Additional intermediate data points would further refine the L/D trend in this location.

Increase in radius ratios of both configurations show the reduction of required $\alpha$ during cruise. The larger radius ratios of both have the lowest required cruise $\alpha$ over the range of ratios examined. Similar to the L/D data, the prescribed radius configuration greatly reduced its required $\alpha$ much more quickly than the dual radius configuration, as radius ratio increased. The prescribed radius configuration also exhibited the slope 
between the last two data points to be approaching zero, suggesting a minimum required $\alpha$ within close proximity of the largest radius ratio of 3721 .

The pitching moment data again shows the drop in moment caused by low airspeed present at the trailing edge of the smallest radius ratios of the two flap configurations. With increase in the radius ratios, the negative pitching moment trends first increase as the flow starts staying at the higher velocities over the entire trailing edge of the flaps and then begins to reduce with further increase of the radius ratios. The prescribed radius flap starts to see a reduction in pitching moment at the largest radius ratio where the dual radius had yet to see a reduction, although the trend suggests a reduction with increasing the radius ratio. 


\section{Chapter 7: Conclusion}

Through the evaluation of the different flap configurations at the various flight conditions of takeoff, approach/landing, and cruise, the various flap parameters were characterized. The shorter flap configurations presented a clear advantage over the longer flap lengths in terms of force augmentation, with the dual radius flaps producing more lift than the corresponding length prescribed radius flaps. Although the larger force augmentation of the shorter flaps and dual radius configurations was accompanied by larger drag and negative pitching moments along with more severe stalling characteristics. The incremental lift generated per drag and moment was best achieved by the longer flap configurations, with the prescribed radius configurations outperforming the corresponding dual radius flaps.

The prescribed radius flap configurations displayed the most promising traits when observing their resulting pressure and skin friction distributions during the takeoff and approach/landing conditions (flap deflections $>0^{\circ}$ ). All prescribed radius flaps presented the favorable trait of reducing the skin friction fluctuation observed at the transition from the $r_{1}$ to $r_{2}$ flap surface on all of the dual radius configurations during slot operation or when the flap is deflected. The fluctuation is a direct cause of the discontinuous surface curvature of the dual radius flaps, and can be alleviated with the smooth transitioning continuous curvature of the prescribed radius configuration.

The longer flap configurations had the best influence on the airfoils cruise performance, encouraging flow attachment over the entire flap surface along with requiring less $\alpha$ to produce a specific target cruise $\mathrm{C}_{1}$. The prescribed radius flaps also 
showed benefit over corresponding dual radius flaps by producing less drag and pitching moment, maintaining less curvature over the flapped surface of the airfoil. Although a fluctuation in both $\mathrm{C}_{\mathrm{p}}$ and $\mathrm{C}_{\mathrm{f}}$ was experienced by the prescribed radius flaps at the transition between the airfoil and flap surface. This was due to the much larger curvature on the exposed portion of the prescribed radius flaps, which match the large $\mathrm{r}_{1}$ curvature when the flap is deflected, to the much smaller curvature of the exposed portion of the dual radius flaps $\left(\mathrm{r}_{2}\right)$. This fluctuation was significantly lessened on the longer prescribed radius flaps that held a more constant curvature over the entirety of the flap. The performance characterization of the various geometric parameters, presented at the end of each results section, displayed trends that are intended to be used in future design and development of CC flap systems.

The alternate flap surface of the prescribed radius displayed several beneficial characteristics for both deployed and retracted conditions, with the most interesting being its effect on the pressure and skin friction over the flap surface. Although this study only investigated specific geometry of the cubically prescribed radius, variations to this geometry are encouraged to continue the research on the effect of surface curvature on CC flap performance. 


\section{Chapter 8: Future Work}

Several different performance characteristics of the flap configurations introduced specific flow features that are of interest. The first distinct feature encountered by all flap configurations was the "dip" or fluctuation in $\mathrm{C}_{\mathrm{f}}$ that occurred at the flap location where $\mathrm{r}_{1}$ transitioned into the second flap surface. This occurrence appeared to be a direct function of the surface curvature of the surface. To investigate this issue further, one could isolate the curved surface alone and study the effects of curvature on the separation location of the flow, pressure distribution, along with skin friction readings. There has been some computational research performed by Wilde ${ }^{103}$ on the variation of curvature, although only reporting slot flow separation locations. Detailed design should be put into the generation of the surface, following guidelines set by previous work ${ }^{20}$ to ensure sufficient CC performance. Additional computational or experimental research on this topic would hold significant insight into the details of Coanda surface performance on CC flap systems.

Another point of interest is the pressure and skin friction fluctuation experienced by only the prescribed radius flaps during transonic cruise when there was no blowing from the slots. Although to the eye, the surface contours of the different flaps are of little difference, the variation of surface curvature of the different flap configurations caused abrupt changes in pressure and skin friction values. These fluctuations in pressure could easily influence separation, greatly degrading the performance of an airfoil. Further research on this subject would be of great value to the design process of supercritical airfoil modification to implement a flap system. 


\section{References}

${ }^{1}$ Englar, R. J., Smith, M. J., Kelley, S. M., and Rover III, R. C., "Application of Circulation Control to Advanced Subsonic Transport Aircraft, Part 1: Airfoil Development," Journal of Aircraft, Vol. 31, No. 5, September-October 1994.

${ }^{2}$ Englar, R. J., "Overview of Circulation Control Pneumatic Aerodynamics: Blown Force and Moment Augmentation and Modification as Applied Primarily to Fixed-Wing Aircraft," NASA/CP-2005-213509/PTI, June 2005.

${ }^{3}$ Englar, R. J., "Subsonic Wind Tunnel Investigation of the High Lift Capability of a Circulation Control Wing on a 1/5 Scale T-2C Aircraft Model," NSRDC Report ASED-299, AD A7781856, David W. Taylor Naval Ship Research and Development Center, Bethesda, MD, May 1973.

${ }^{4}$ Englar, R. J., "Investigation into Application of the High Velocity Circulation Control Wall Jet for High Lift and Drag Generation on STOL Aircraft," AIAA Paper 74502, November 1976.

${ }^{5}$ Englar, R. J., Trobaugh, L. A., and Hemmerly, R. A., "STOL Potential of the Circulation Control Wing for High-Performance Aircraft," Journal of Aircraft, Vol. 15, No. 3, March 1978.

${ }^{6}$ Harris, M. J., "Investigation of a Pneumatic Thrust Deflector Based on Circulation Control Technology," Report DTNSRDC/ASED-83/01, AD A128-950, David W. Taylor Naval Ship Research and Development Center, Bethesda, MD, February 1983. 
${ }^{7}$ California Polytechnic State University San Luis Obispo, Georgia Tech Research Institute, and DHC Engineering, "The Integrated Modeling and Verification of a Hybrid Wing-Body Low Noise CESTOL Aircraft," NASA Contract \#NNL07AA55C, October 2008.

${ }^{8}$ Wood, N., and Nielson, J. "Circulation Control Airfoils Past, Present, and Future," AIAA Paper 850204, January 1985.

${ }^{9}$ de la Montanya, J., "Circulation Control: Past to Present," NASA/Cal Poly ESTOL Project Team, September 2005.

${ }^{10}$ Loth, J. L., and Boasson, M., "Circulation Control Optimization," Paper AIAA-830082, AIAA $21^{\text {st }}$ Aerospace Sciences Meeting, Reno, NV, 10-13 January 1983.

${ }^{11}$ Davidson, I. M., "Aerofoil Boundary-Layer Control System," British Patent No. 913754, 1960.

${ }^{12}$ Englar, R. J., "Experimental Investigation of the High Velocity Coanda Wall Jet Applied to Bluff Trailing Edge Circulation Control Airfoils," M. S. Thesis, University of Maryland, Department of Aerospace Engineering, June 1973.

${ }^{13}$ Abramson, J., "Two-Dimensional Subsonic Wind Tunnel Evaluation of a 20-PercentThick Circulation Control Airfoil,” NSRDC Report ASED-331, AD A027-164, David W. Taylor Naval Ship Research and Development Center, Bethesda, MD, June 1975.

${ }^{14}$ Chang, P. A. III, Slomski, J., Marino, T., Ebert, M. P., and Abramson, J., "FullReynolds Stress Modeling of Circulation Control Airfoils," Naval Surface Warfare Center - Carderock Division, West Bethesda, MD, March 2004. 
${ }^{15}$ Englar, R. J., "Application of Advanced Aerodynamic Technology to Ground and Sport Vehicles," AAIA 2008-6731, Georgia Tech Research Institute, Georgia Institute of Technology, Atlanta, GA, August 2008.

${ }^{16}$ Englar, R. J., "Two-Dimensional Subsonic Wind Tunnel Tests of Two 15-PercentThick Circulation Control Airfoils,” NSRDC Report ASED-211, AD A900-210, David W. Taylor Naval Ship Research and Development Center, Bethesda, MD, August 1971.

${ }^{17}$ Englar, R. J., "Final Report: Summary of Research: Continued Development and Application of Circulation Control Pneumatic Technology to Advanced Transport Aircraft," NASA/CR-1998-207471, March 1998.

${ }^{18}$ Loth, J. L., "Circulation Control STOL Aircraft Design Aspects," Paper N88-17610, Mechanical and Aerospace Engineering, West Virginia University, 1988.

${ }^{19}$ Waters, M. H., and Hall, D. W., "Configuration Development Discussion - DRAFT," Empirical Systems Aerospace, Pismo Beach, CA, March 2008.

${ }^{20}$ Englar, R. J., and Williams, R. M., "Design of a Circulation Control Stern Plane for Submarine Applications," NSRDC TN AL-200, David W. Taylor Naval Ship Research and Development Center, Bethesda, MD, March 1971.

${ }^{21}$ Furey, R. J., and Whitehead, R. E., "Static Evaluation of a Circulation Control Centrifugal Fan,” DTNSRDC Report 77-0051, AD A041-463, David W. Taylor Naval Ship Research and Development Center, Bethesda, MD, June 1977.

22 Williams, R. M., "Some Research on Rotor Circulation Control," Third CAL/AVLABS Symposium on Aerodynamics of Rotary Wing and V/STOL 
Aircraft, Vol. 2 of Proceedings, Cornell University, Buffalo, NY, 18-20 June 1969.

${ }^{23}$ Williams, R. M., "Recent Developments in Circulation Control Rotor Technology," Meeting of Advisory Group for Aerodynamics R\&D (Aerodynamics of Rotary Wings), AGARD-CPP-111, Marseilles, France, 13-15 September 1972.

${ }^{24}$ Englar, R. J. and Williams, R. M., “Test Techniques for High Lift Two-Dimensional Airfoils with Boundary Layer and Circulation Control for Application to Rotary Wing Aircraft," Canadian Aeronautics and Space Journal, Vol. 19, No. 3, March 1973, pp. 93-108.

${ }^{25}$ Wilkerson, J. B. et al., "The Application of Circulation Control Aerodynamics to a Helicopter Rotor Model," Paper 28 presented at $29^{\text {th }}$ Annual Forum of American Helicopter Society, Washington, D.C., 10-11 May 1973.

${ }^{26}$ Williams, R. M., “Application of Circulation Control Rotor Technology to a Stopped Rotor Aircraft Design,” Presented at the First European Rotorcraft and Powered Lift Aircraft Forum, Southampton, England, 22-24 September 1975.

${ }^{27}$ Williams, R. M., Leitner, R. T., and Rogers, E. O., "X-Wing: A New Concept in Rotary Wing VTOL," Presented at the American Helicopter Society Symposium on Rotor Technology, Philadelphia, PA, August 1976.

${ }^{28}$ Anonymous, "X-Wing Full Scale Wind Tunnel Test Final Report," Lockheed Report SP-5190, October 1980.

29 "X-Wing Research Vehicle," NASA Dryden Flight Research Center, URL: http://www.dfrc.nasa.gov/gallery/photo/X-Wing/HTML/EC86-33555-2.html, cited June 2010. 
${ }^{30}$ Englar, R. J., "Two-Dimensional Transonic Wind Tunnel Tests of Three 15-Percent Thick Circulation Control Airfoils," Technical Note AL-182, David W. Taylor Naval Ship Research and Development Center, Bethesda, MD, December 1970.

${ }^{31}$ Shovlin, M. D., Englar, R. J., Eppel, J. C., and Nichols, J. H., "Large-Scale Static Investigation of Circulation-Control-Wing Concepts Applied to Upper-SurfaceBlowing Aircraft," NASA Technical Paper No. 2684, January 1987.

${ }^{32}$ Loth, J. L., "Some Aspects of STOL Aircraft Aerodynamics," Business Aircraft Meeting, Paper No. 730328, Wichita, KS, 3-6 April 1973.

${ }^{33}$ Nichols, J. H., Jr., and Englar, R. J., “Advanced Circulation Control Wing System for Navy STOL Aircraft," Paper AIAA-80-1825, Presented at AIAA Aircraft Systems Meeting, Anaheim, CA, 4-6 August 1980.

${ }^{34}$ Englar, R. J., et al., "Design of the Circulation Control Wing STOL Demonstrator Aircraft," AIAA-79-1842R, Journal of Aircraft, Vol. 18, No. 1, January 1981.

${ }^{35}$ Nichols, J. H., Jr., et al., "Experimental Development of an Advanced Circulation Control Wing System for Navy STOL Aircraft,” Paper AIAA-81-0151, Presented at AIAA $19^{\text {th }}$ Aerospace Sciences Meeting, St. Louis, MO, 12-19 January 1981.

${ }^{36}$ Harris, M. J., "Investigation of the Circulation Control Wing/Upper Surface Blowing High-Lift System on a Low Aspect Ratio Semi span Model," Report DTNSRDC/ASED-81/10, AD A103-090, David W. Taylor Naval Ship Research and Development Center, Bethesda, MD, May 1981.

${ }^{37}$ Englar, R. J., "Development of the CCW/Supercritical No-Moving-Parts High Lift Airfoil," Presented at AFSC/NAVMAT Science and Engineering Symposium, Wright-Patterson AFB, OH, 27-29 October 1981. 
${ }^{38}$ Wellman, L. K., and Jacobsen, C., "Wind Tunnel Investigation of the Application of Circulation Control to a Forward Swept Wing," Report DTNSRDC/ASED-82/05, AD 068-714, David W. Taylor Naval Ship Research and Development Center, Bethesda, MD, June 1982.

${ }^{39}$ Eppel, J. C. et al., and Englar, R. J., and Nichols, J. H., Jr., "Static Investigation of the Circulation-Control-Wing/Upper-Surface-Blowing Concept Applied to the Quite Short-Haul Research Aircraft,” Report NASA-TM-84232, July 1982.

${ }^{40}$ Yang, H. S. D., and Nichols, J. H., Jr., "Design Integration of CCW/USB for a SeaBased Aircraft," Paper ICAS-82-1.6.1, Presented at $13^{\text {th }}$ Congress of International Council of the Aeronautical Sciences, AIAA Aircraft Systems and Technology Conference, Seattle, WA, 22-27 August 1982.

${ }^{41}$ Salikuddin, M. et al., "Noise from a Circulation Control Wing with Upper Surface Blowing," Journal of Aircraft, Vol. 24, No. 1, 1987, pp. 55-64.

${ }^{42}$ Englar, R. J., "Development of Circulation Control Technology for Powered-Lift Aircraft," Paper No. N88-17608, Advanced Flight Sciences Department, Lockheed-Georgia Company, 1986.

${ }^{43}$ Riddle, D. W., and Eppel, J. C., "A Potential Flight Evaluation of an Upper-SurfaceBlowing/Circulation-Control-Wing Concept," NASA-Ames Research Center, Paper No. N88-17609, 1986.

${ }^{44}$ Loth, J. L., "Advantages of Combining BLC Suction with Circulation Control HighLift Generation," AIAA Applications of Circulation Control Technology Vol. 214, West Virginia University, Morgantown, WV, 2005. 
${ }^{45}$ Englar, R. J., "Subsonic Two-Dimensional Wind Tunnel Investigations of the High Lift Capability of Circulation Control Wing Sections," NSRDC Report ASED-274, AD A024-346, David W. Taylor Naval Ship Research and Development Center, Bethesda, MD, April 1975.

${ }^{46}$ Englar, R. J., "Development of the A-6/Circulation Control Wing Flight Demonstrator Configuration,” Report DTNSRDC/ASED-79/01, AD A081-241, David W. Taylor Naval Ship Research and Development Center, Bethesda, MD, January 1978.

${ }^{47}$ Mayfield, J., "Circulation Control Wing Demonstrates Greater Lift," Aviation Week \& Space Technology, 1979.

${ }^{48}$ Englar, R. J., "Low-Speed Aerodynamic Characteristics of a Small Fixed-TrailingEdge Circulation Control Wing Configuration Fitted to a Supercritical Airfoil," Report DTNSRDC/ASED-81/08, AD A101-540, David W. Taylor Naval Ship Research and Development Center, Bethesda, MD, March 1981.

${ }^{49}$ Englar, R. J., and Huson, G. G., "Development of Advanced Circulation Control Wing High-Lift Airfoils," David W. Taylor Naval Ship Research and Development Center, Bethesda, MD, January 1983.

${ }^{50}$ Jones, G. S., Lin, J. C., Allan, B. G., Milholen, W. E., Rumsey, C. L., and Swanson, R. C., "Overview of CFD Validation Experiments for Circulation Control Applications at NASA," AIAA Paper 2008-030041, NASA Langley Research Center, Hampton, VA, 2008. 
${ }^{51}$ Chang, P. A., Slomski, J., Marino, T., and Ebert, M. P., "Numerical Simulation of Two- and Three-Dimensional Circulation Control Problems," $43^{\text {rd }}$ AIAA Aerospace Sciences Meeting and Exhibit, 2005.

${ }^{52}$ Lee-Rausch, E. M., Vatsa, V. N., and Rumsey, C. L., "Computational Analysis of Dual Radius Circulation Control Airfoils," AIAA Paper 2006-3012, 2006.

${ }^{53}$ Liu, Y., "Numerical Simulation of the Aerodynamic Characteristics of Circulation Control Wing Sections," Ph. D. Dissertation, School of Aerospace Engineering, Georgia Institute of Technology, Atlanta, GA, 2003.

${ }^{54}$ Baker, W. J., and Paterson, E. G., "RANS CFD Simulation of a Circulation-Control Foil: Validation of Performance, Flow Field, and Wall Jet," Paper AIAA 20063010, Presented at The $3^{\text {rd }}$ AIAA Flow Control Conference, San Francisco, CA, 5-8 June 2006.

55 Swanson, R. C., and Rumsey, C. L., "Numerical Issues for Circulation Control Calculations," Paper AIAA 2006-3008, Presented at The $3^{\text {rd }}$ AIAA Flow Control Conference, San Francisco, CA, 5-8 June 2006.

${ }^{56}$ Swanson, R. C., Rumsey, C. L., and Anders, S. G, "Progress Towards Computational Method for Circulation Control Airfoils," AIAA Paper 2005-29, 2005.

${ }^{57}$ Pfingsten, K. C., Jensch, C., Körber, K. W., and Radespiel, R., "Numerical Simulation of the Flow Around Circulation Airfoils," First CEAS European Air and Space Conference, 2007.

${ }^{58}$ Bell, B., "Turbulent Flow Case Studies," Fluent Services Center, 2003. 
${ }^{59}$ Storm, T. M., and Marshall, D. D., "Assessing the $\mathrm{v}^{2}-f$ Turbulence Models for Circulation Control Applications," AIAA Paper 2010-1054, 48th AIAA Aerospace Sciences Meeting and Exhibit, Orlando, FL, 4-7 January 2010.

${ }^{60}$ Paterson, E. G., and Baker, W. J., "Simulation of Steady Circulation Control for Marine-Vehicle Control Surfaces," Paper AIAA 2004-748, Presented at The $42^{\text {nd }}$ AIAA Aerospace Sciences Meeting, Reno, NV, 5-8 January 2004.

${ }^{61}$ McGown, G., and Gopalarathnam, A., "CFD Analysis of a Circulation Control Airfoil Using FLUENT," Mechanical and Aerospace Engineering Department, North Carolina State University, Raleigh, North Carolina, 2004.

${ }^{62}$ Liu, Y., et al., "Computational Evaluation of the Steady and Pulsed Jet Effects on the Performance of a Circulation Control Wing Section," NASA/CP-2005-213509, NASA/ONR Circulation Control Workshop, 16-17 March 2004, pp. 295-336.

${ }^{63}$ Swanson, R. C., "Aspects of Numerical Simulation of Circulation Control Airfoils," NASA/CP-2005-213509, NASA/ONR Circulation Control Workshop, 16-17 March 2004, pp. 227-273.

${ }^{64}$ Loth, J. L., "Why Have Only Two Circulation Control STOL Aircraft Been Built and Flown in Years 1974-2004," NASA/CP-2005-213509, NASA/ONR Circulation Control Workshop, 16-17 March 2004, pp. 603-640.

${ }^{65}$ Loth, J. L., and Roberts, S. C., "Flight Performance of a Circulation Controlled STOL Aircraft," Journal of Aircraft, Vol. 18, No. 3, 1976, pp. 169-173.

${ }^{66}$ Hemmerly, R. A., "An Investigation of the Performance of a J-52-P-8A Engine Operating Under the Influence of High Bleed Flow Extraction Rates," 
DWTNSRDC Report ASED-387, AD A057-325, David W. Taylor Naval Ship Research and Development Center, Bethesda, MD, August 1977.

${ }^{67}$ Pugliese, A. J., and Englar, R. J., "Flight Testing the Circulation Control Wing," Paper AIAA-79-1791, Presented at AIAA Aircraft Systems and Technology Meeting, New York, 20-22 August 1979.

${ }^{68}$ Nichols, J. H., Jr., "Development for High Lift Devices for Application to Advanced Navy Aircraft," Report DTNSRDC-80/058, AD A084-226, David W. Taylor Naval Ship Research and Development Center, Bethesda, MD, April 1980.

${ }^{69}$ Mayfield, J., "Navy Sponsors Coanda Rotor Program," Aviation Week \& Space Technology, March 1980, pp. 69-74.

${ }^{70}$ Wilkerson, J. B., and Linck, D. W., "A Model Rotor Performance Validation for the CCR Technology Demonstrator," Preprint 902 presented at $31^{\text {st }}$ Annual National Forum of the American Helicopter Society, Washington, D.C., May 1975.

${ }^{71}$ Abramson, J., and Rogers, E. O., "High-Speed Characteristics of Circulation Control Airfoils," AIAA-83-0265, David W. Taylor Naval Ship Research and Development Center, Bethesda, MD, January 1983.

${ }^{72}$ Tai, T. C., and Kidwell, G. H., Jr., "Numerical Optimization of Circulation Control Airfoils," Paper AIAA-81-0016, Presented at AIAA $19^{\text {th }}$ Aerospace Sciences Meeting, St. Louis, MO, 12-15 January 1981.

${ }^{73}$ Collier, F., “Overview of NASA's Environmentally Responsible Aviation (ERA) Project," N+2 Advanced Vehicle Concepts \& Quick-Starts NRA Pre-Proposal Meeting, February 19, 2010. 
${ }^{74}$ Rich, P., McKinley, R. J., and Jones, G. S., "Circulation Control in NASA's Vehicle Systems," NASA CP-205-213509 (part 1), June 2005.

${ }^{75}$ Munro, S. E., Ahuja, K. K., and Englar, R. J., "Noise Reduction Through Circulation Control," AIAA-2001-0666, Georgia Institute of Technology, GTRI/ATASL, Atlanta, GA, January 2008.

${ }^{76}$ Harris, C. D., "NASA Supercritical Airfoils - A Matrix of Family-Related Airfoils," NASA TP-2969, NASA Langley Research Center, Hampton, VA, 1990.

${ }^{77}$ Englar, R. J., "Characteristics and Development of the Circulation Control Airfoil," Proceedings of Advanced Technology Airfoil Research Conference, NASA Conference, Publication 2046, Hampton, VA, March 7-9, 1978.

${ }^{78}$ Joslin, R. D., and Jones, G. S., "Application of Circulation Control Technology", Progress in Astronautics and Aeronautics, Vol. 212, May 2006.

${ }^{79}$ Jones, G. S., and Joslin, R. D., Proceedings of the NASA/ONR 2004 Circulation Control Workshop, NASA CP-2005-213509 (parts 1 and 2), June 2005.

${ }^{80}$ Jones, G. S., Yao, C. S., and Allan, B. G., "Experimental Investigation of a 2D Supercritical Circulation-Control Airfoil Using Particle Image Velocimetry," AIAA Paper 2006-3009, June 2006.

${ }^{81}$ Marcos, J. M., "Computational and Experimental Comparison of a Powered Lift, Upper Surface Blowing Configuration," AIAA-2010-502, 48th AIAA Aerospace Sciences Meeting and Exhibit, Orlando, FL, January 4-7, 2009.

${ }^{82}$ Linton, S. W., "Computation of the Post stall Behavior of a Circulation Control Airfoil," Journal of Aircraft, Vol. 31, No. 6, 1994, pp. 1273-1280. 
${ }^{83}$ Storm, T. M., "Assessing the $\mathrm{v}^{2}-f$ Turbulence Model for Circulation Control Applications," M.S. Thesis, Aerospace Engineering Department, California Polytechnic State University, San Luis Obispo, CA, April 2010.

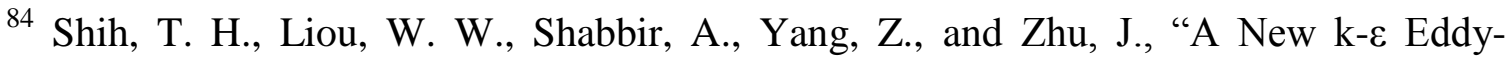
Viscosity Model for High Reynolds Number Turbulent Flows - Model Development and Validation," Computers \& Fluids, Vol. 24, No. 3, 1995, pp.227-238.

${ }^{85}$ Golden, R. M., and Marshall, D. D., "Design and Performance of Circulation Control Flap Systems," AIAA 2010-1053, $48^{\text {th }}$ AIAA Aerospace Sciences Meeting and Exhibit, January 4-7, 2010.

${ }^{86}$ Tannehill, J. C., Anderson, D. A., and Pletcher, R. H., Computational Fluid Mechanics and Heat Transfer, Second Edition, Taylor \& Francis Publishing, Philadelphia, PA, 1997.

87 von Kármán, Th., "Mechanical Similitude and Turbulence," NACA Technical Memorandum No. 611,Nachrichten von der Gesellschaft der Wissenschaften zu Göttingen, March 1930.

${ }^{88}$ Kline, S. J., Morkovin, M. V., Sovran, G., and Cockrell, D. J., "Computation of Turbulent Boundary Layers," 1968 AFOSR-IFP-Stanford Conference, Vol. 1, Thermo sciences Division, Stanford University, CA, 1969.

89 “External Flows (Boundary Layers \& Drag)," CEE 3310 Lecture Notes, School of Civil and Environmental Engineering, Cornell University, Ithaca, NY, URL: http://ceeserver.cee.cornell.edu/eac20/cee331/LectureNotes_10/CEE331_34.pdf, cited September 2010. 
${ }^{90}$ Harlow, F. H., and Nakayama, P. I., "Transport of Turbulence Energy Decay Rate," Los Alamos, Science Laboratory, LA-3854, 1968.

91 Launder, B. E., and Spalding, D. B., "Lectures in Mathematical Models of Turbulence," Academic Press, London, 1972.

92 Yakhot, V., and Orszag, S. A., "Renormalization Group Analysis of Turbulence: I. Basic Theory," Journal of Scientific Computing, Vol. 1, No. 1, 1986, pp. 1-51.

93 "Realizable k-epsilon Model," CFD Online, URL: http://www.cfdonline.com/Wiki/Realisable_k-epsilon_model, cited September 2010.

94 Spalart, P. R., and Allmaras, S. R., "A One-Equation Turbulence Model for Aerodynamic Flows," AIAA Journal, Vol. 26, 1988, pp. 1299-1310.

95 Wilcox, D. C., "Reassessment of the Scale-Determining Equation of Advanced Turbulence Models," AIAA Journal, Vol. 26, 1988, pp. 1299-1310.

96 Menter, F. R., “Two-Equation Eddy-Viscosity Turbulence Models for Engineering Applications," AIAA Journal, Vol. 32, No. 8, 1994, pp.1598-1605.

${ }^{97}$ Ansys Inc., and Lebanon, NH, FLUENT 6.3 Documentation, $6^{\text {th }}$ ed., 2008.

${ }^{98}$ Celik, I. B., Ghia, U., Roache, P. J., and Freitas, C. J., "Procedure for Estimation and Reporting of Uncertainty Due to Discretization in CFD Applications," Mechanical and Aerospace Engineering Department, West Virginia University, Morgantown, WV, 2007.

${ }^{99}$ Menter, F. R., “Zonal Two Equation k- $\omega$ Turbulence Models for Aerodynamic Flows,” AIAA-93-2906, 24 ${ }^{\text {th }}$ Fluid Dynamics Conference, July 1993.

100 Englar, R. J., "Circulation Control for High Lift and Drag Generation on STOL Aircraft,” AIAA Journal of Aircraft, Vol.12, No. 5, May 1975, pp. 457-463. 
${ }^{101}$ Lan, C. E., and Roskam, J., "Airplane Aerodynamics and Performance," Design, Analysis and Research Corporation, Lawrence, Kansas, 2003.

${ }^{102}$ Frith, S. P., and Wood, N. J., "The Use of Circulation Control for Flight Control," NASA/CP-2005-213509, NASA/ONR Circulation Control Workshop, 16-17 March 2004, pp. 657-688.

103 Wilde, D., "Analysis of Curvature Effects on Boundary Layer Separation and Turbulence Model Accuracy for Circulation Control Applications," Senior Project, Aerospace Engineering Department, California Polytechnic State University, San Luis Obispo, CA, June 2010. 


\section{Appendix A: Additional Figures}


Table A-1: $60^{\circ}$ flap streamlines and contours of Mach at $\alpha=-5^{\circ}, 0^{\circ}, 3^{\circ}$

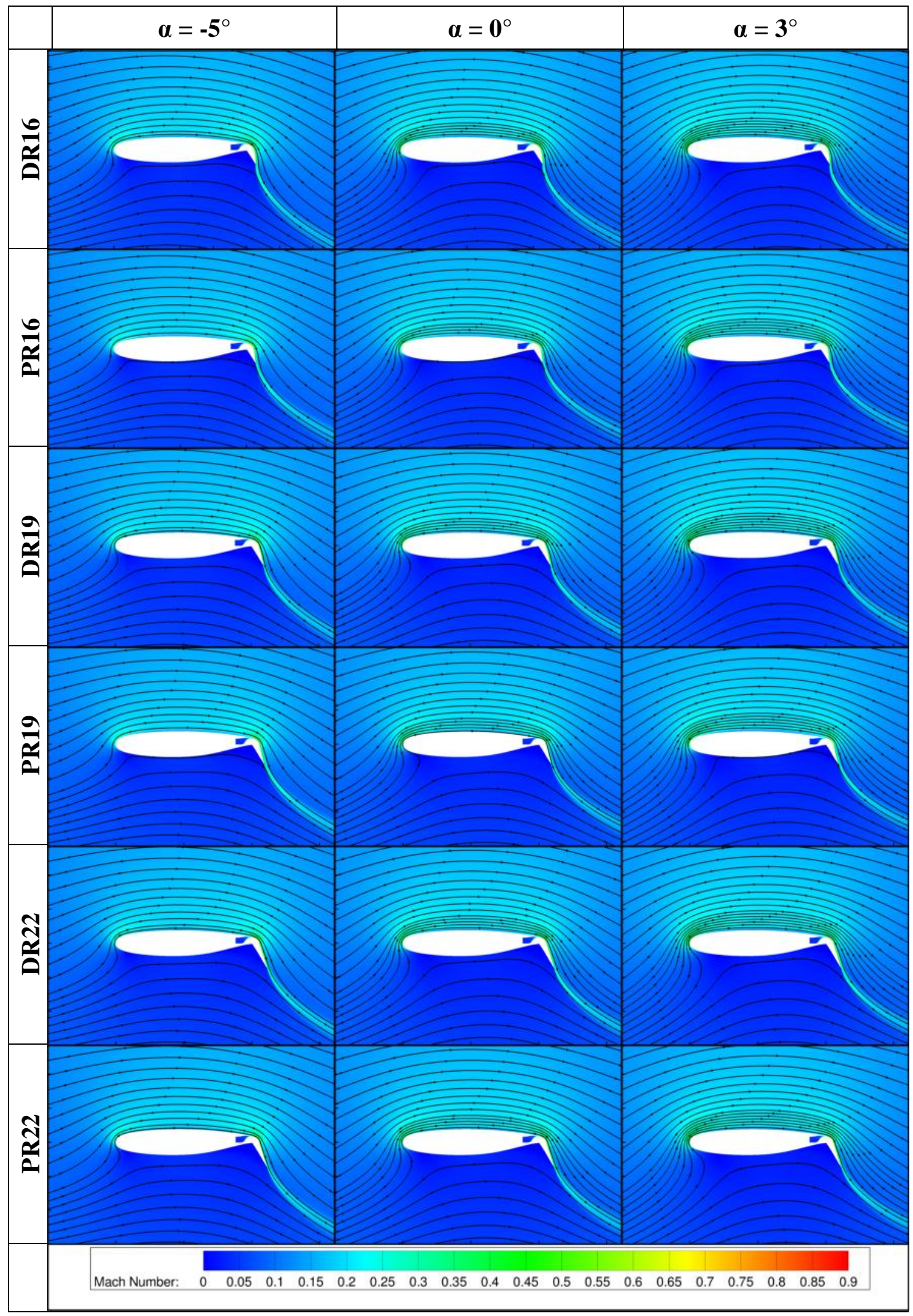




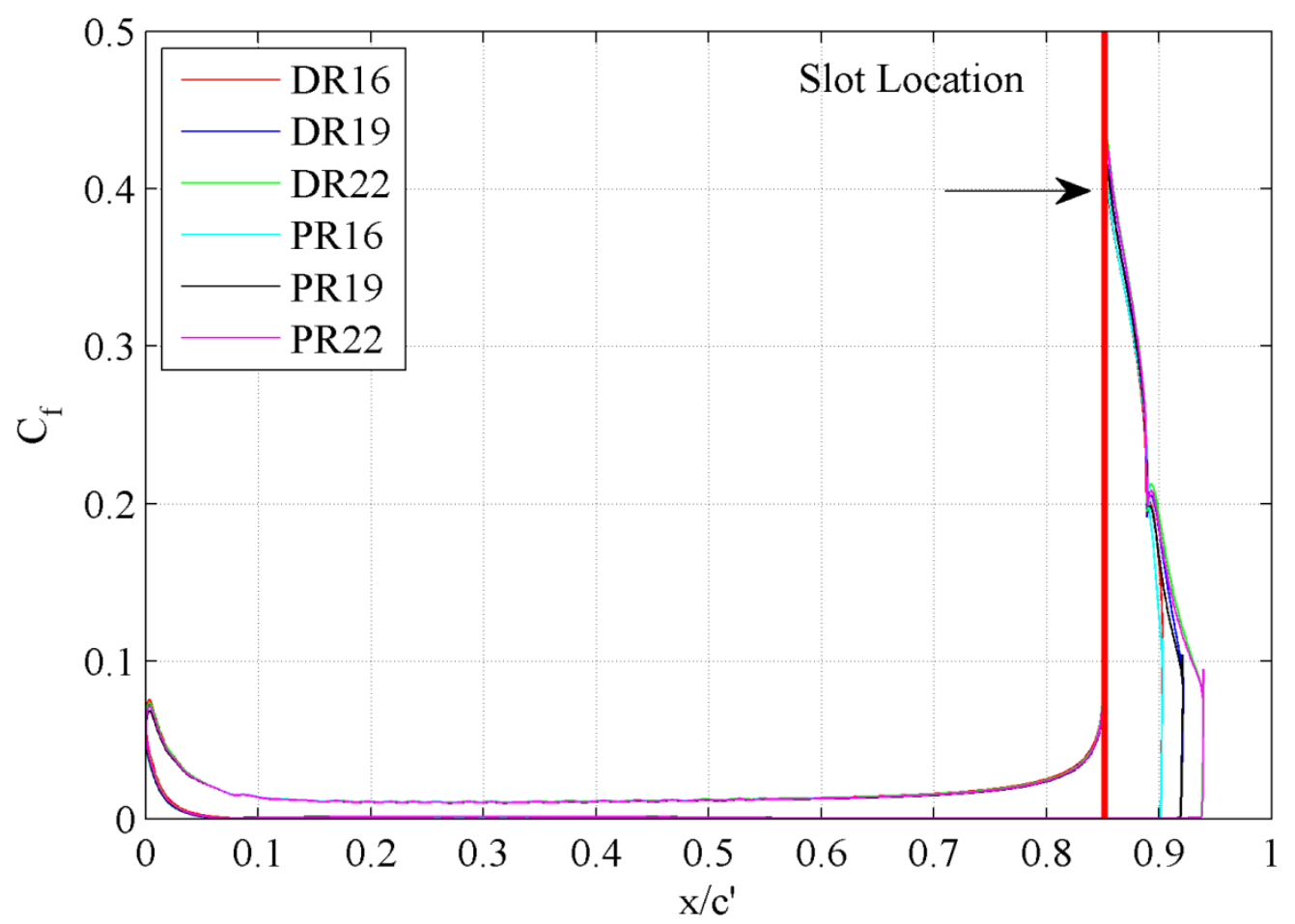

Figure A-9-1: Skin friction distributions for $\alpha=-5^{\circ}, 60^{\circ}$ flaps

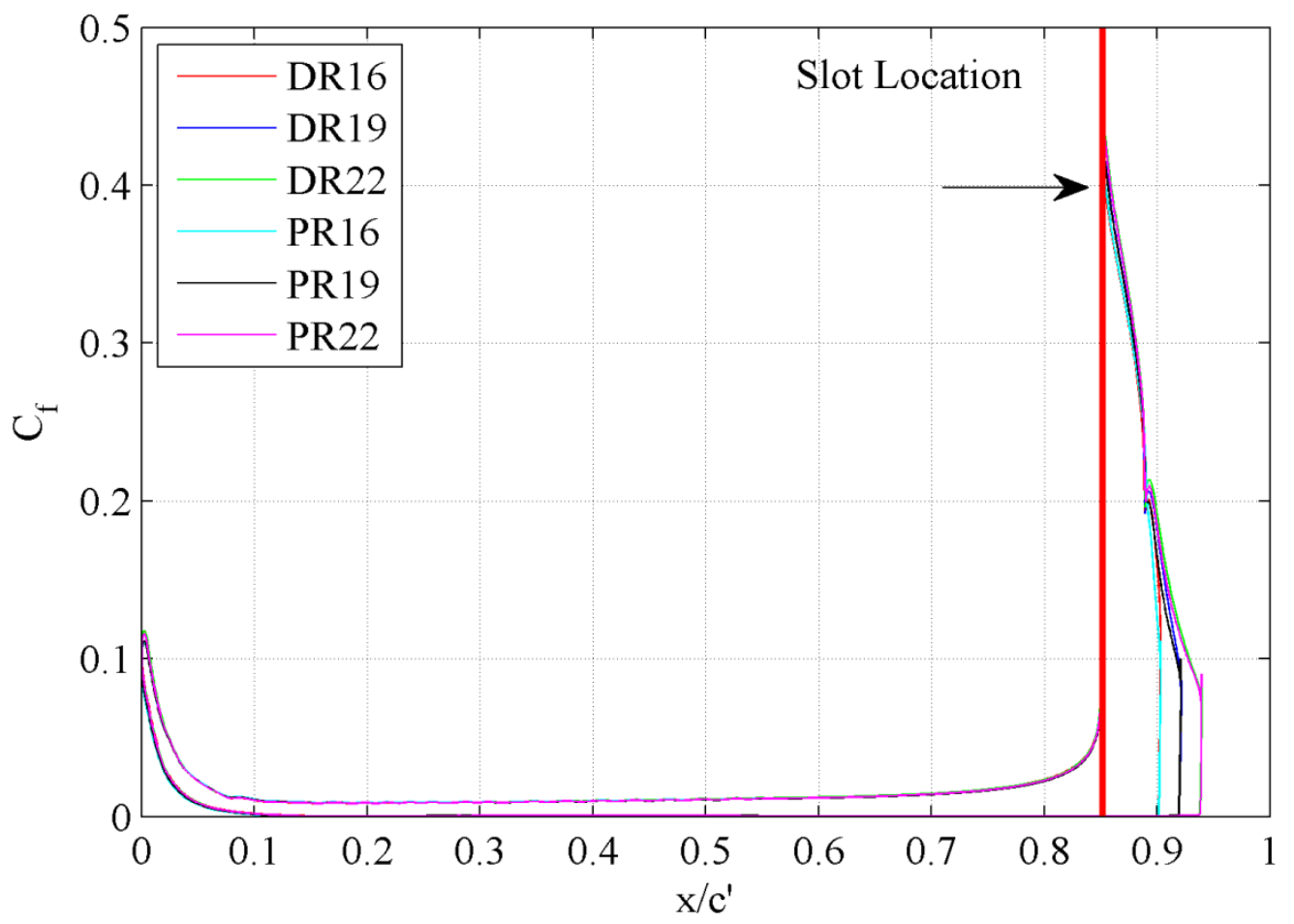

Figure A-9-2: Skin friction distributions for $\alpha=3^{\circ}, 60^{\circ}$ flaps 


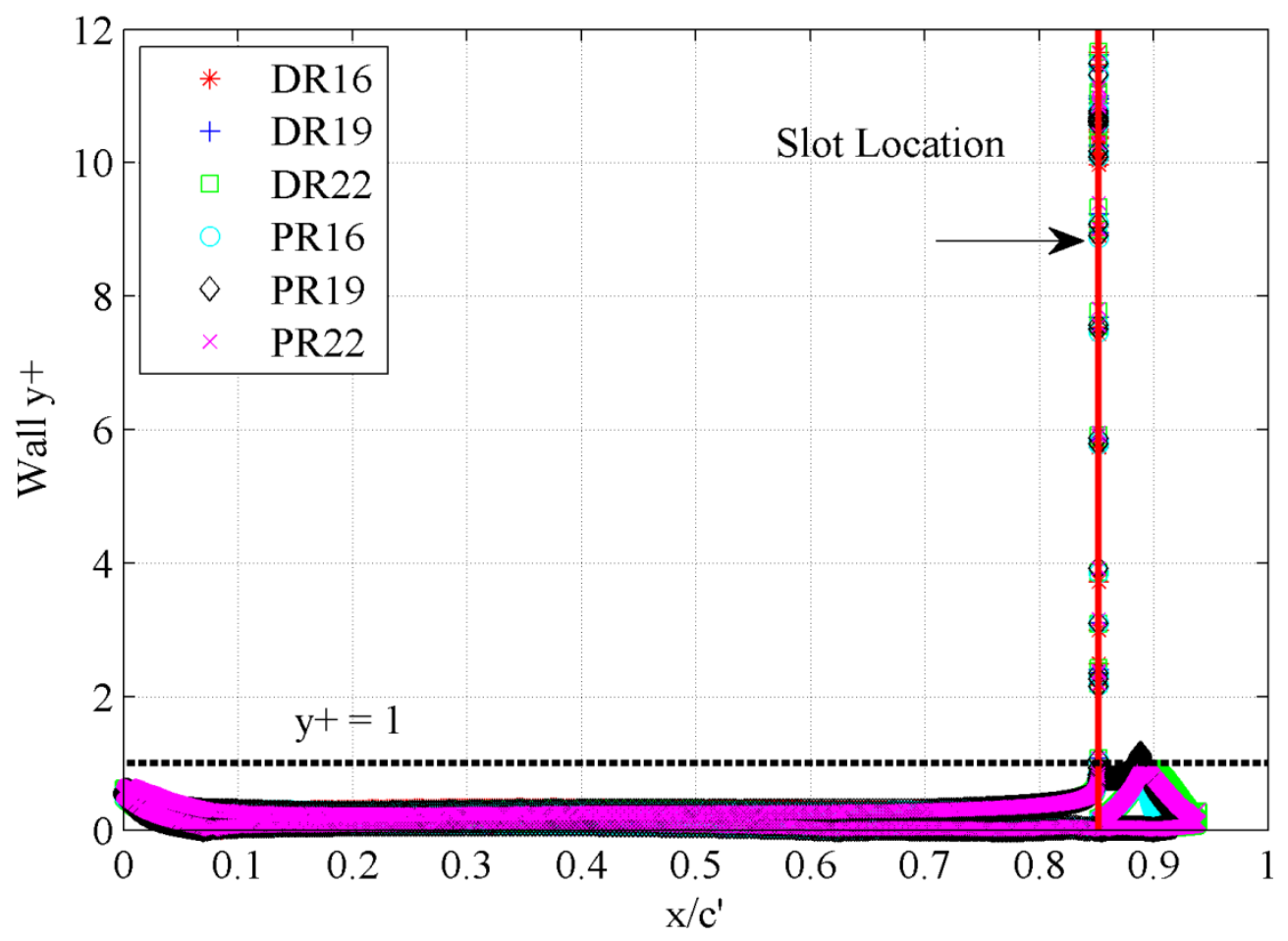

Figure A-9-3: $y^{+}$values of the airfoil/flap configurations, $\alpha=-5^{\circ}, 60^{\circ}$ flaps

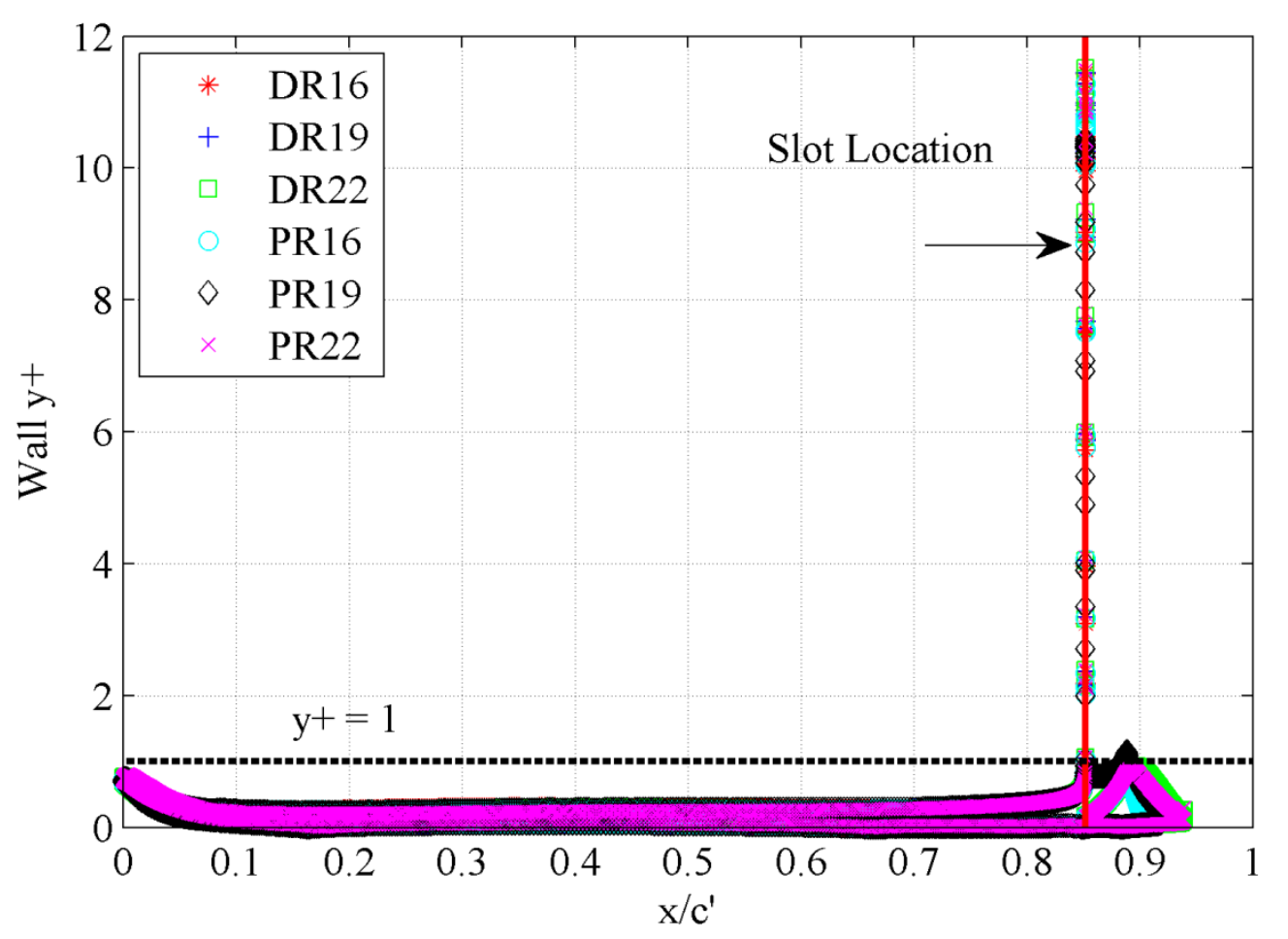

Figure A-9-4: $y^{+}$values of the airfoil/flap configurations, $\alpha=3^{\circ}, 60^{\circ}$ flaps 
Table A-2: $90^{\circ}$ flap streamlines and contours of Mach at $\alpha=-5^{\circ}, 0^{\circ}, 3^{\circ}$

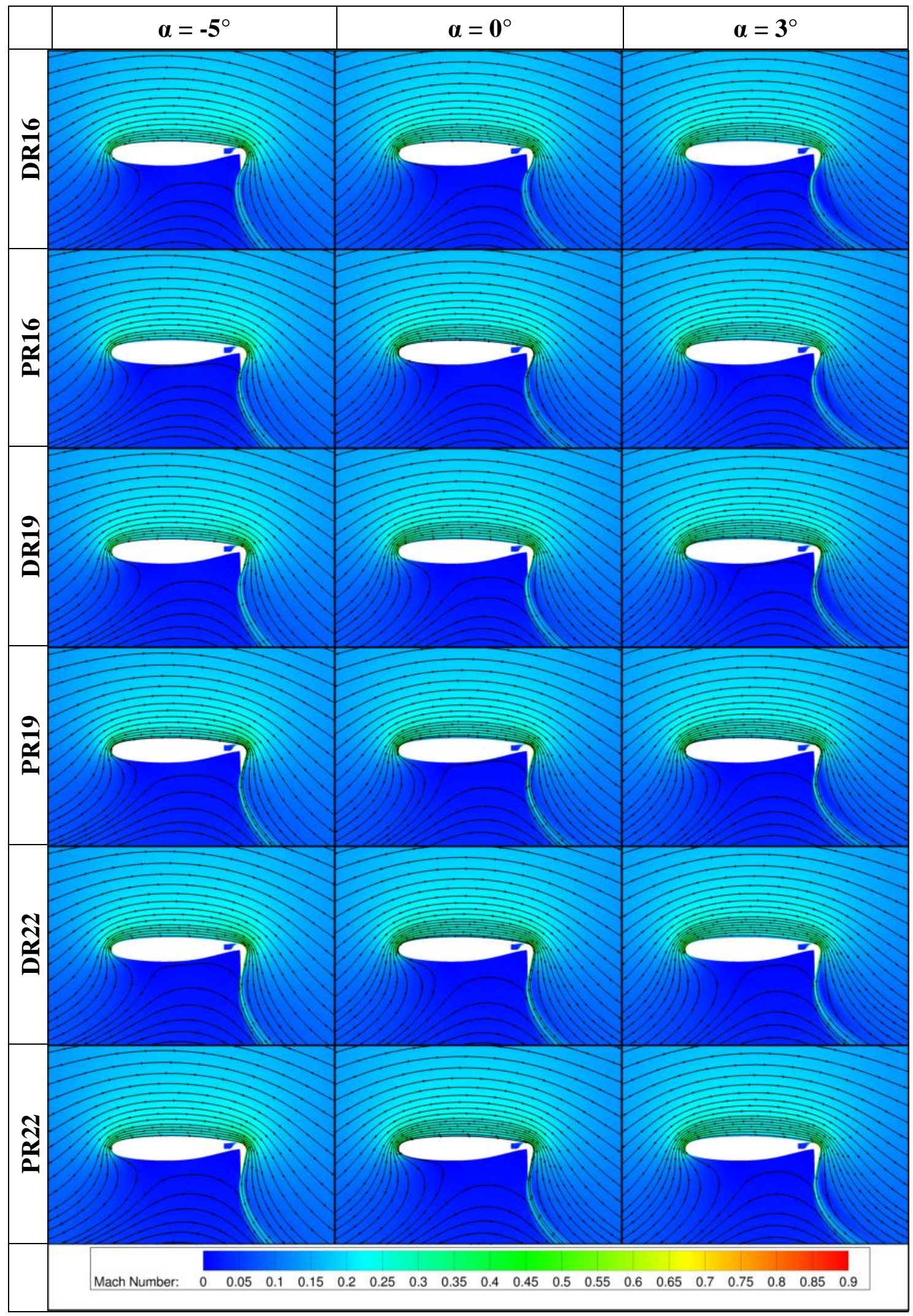




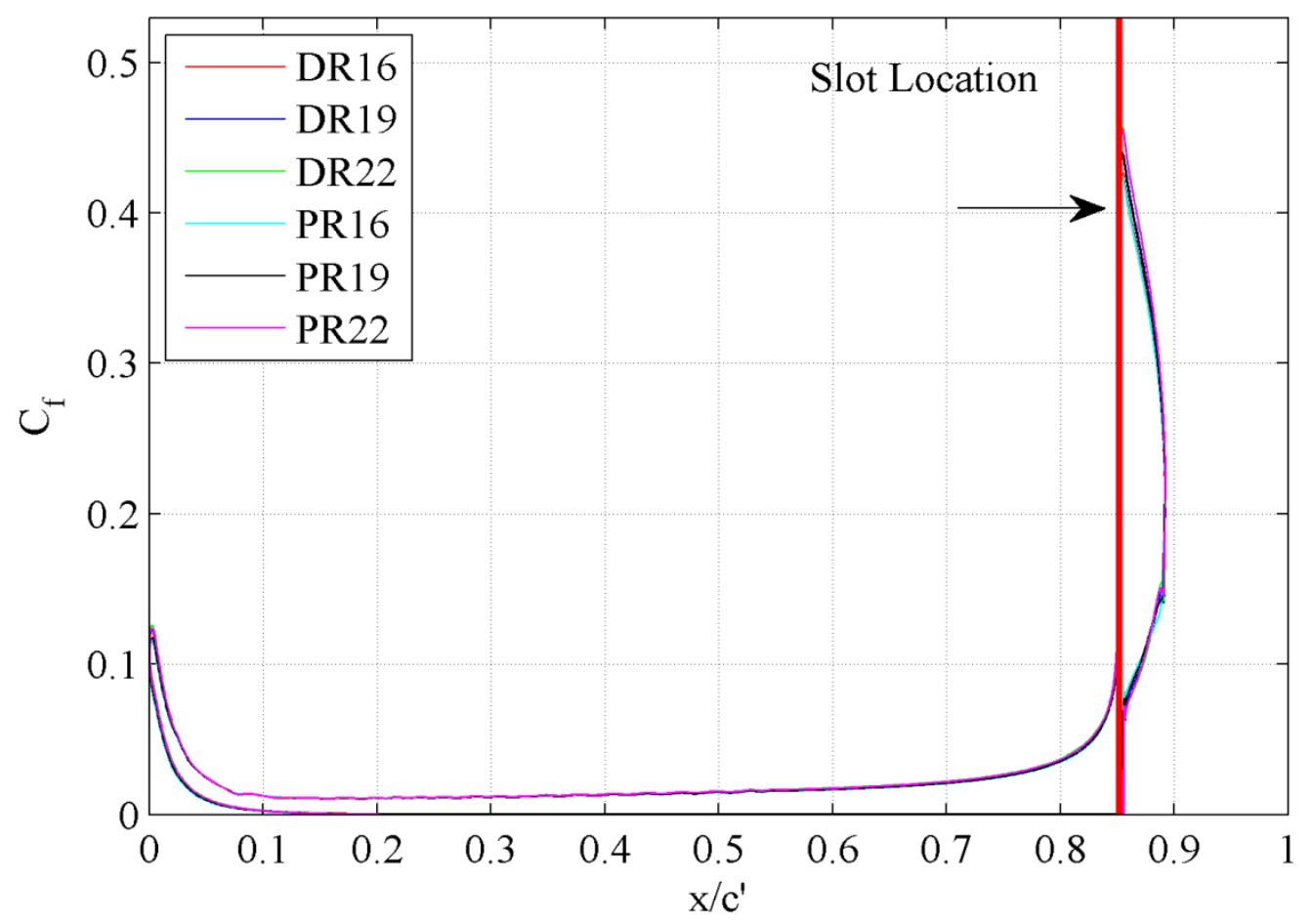

Figure A-9-5: Skin friction distributions for $\alpha=-5^{\circ}, 90^{\circ}$ flaps

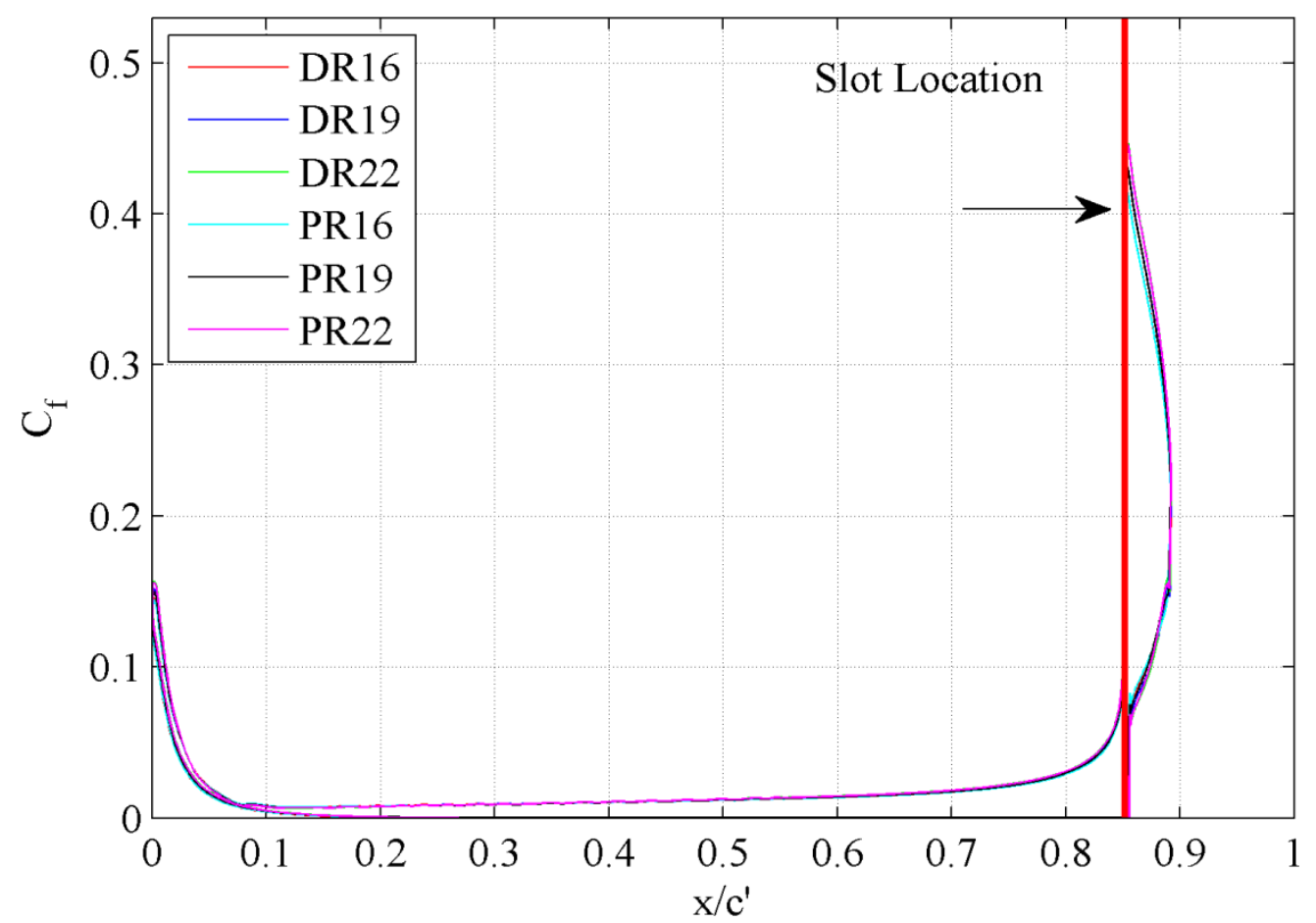

Figure A-9-6: Skin friction distributions for $\alpha=3^{\circ}, 9^{\circ}$ flaps 


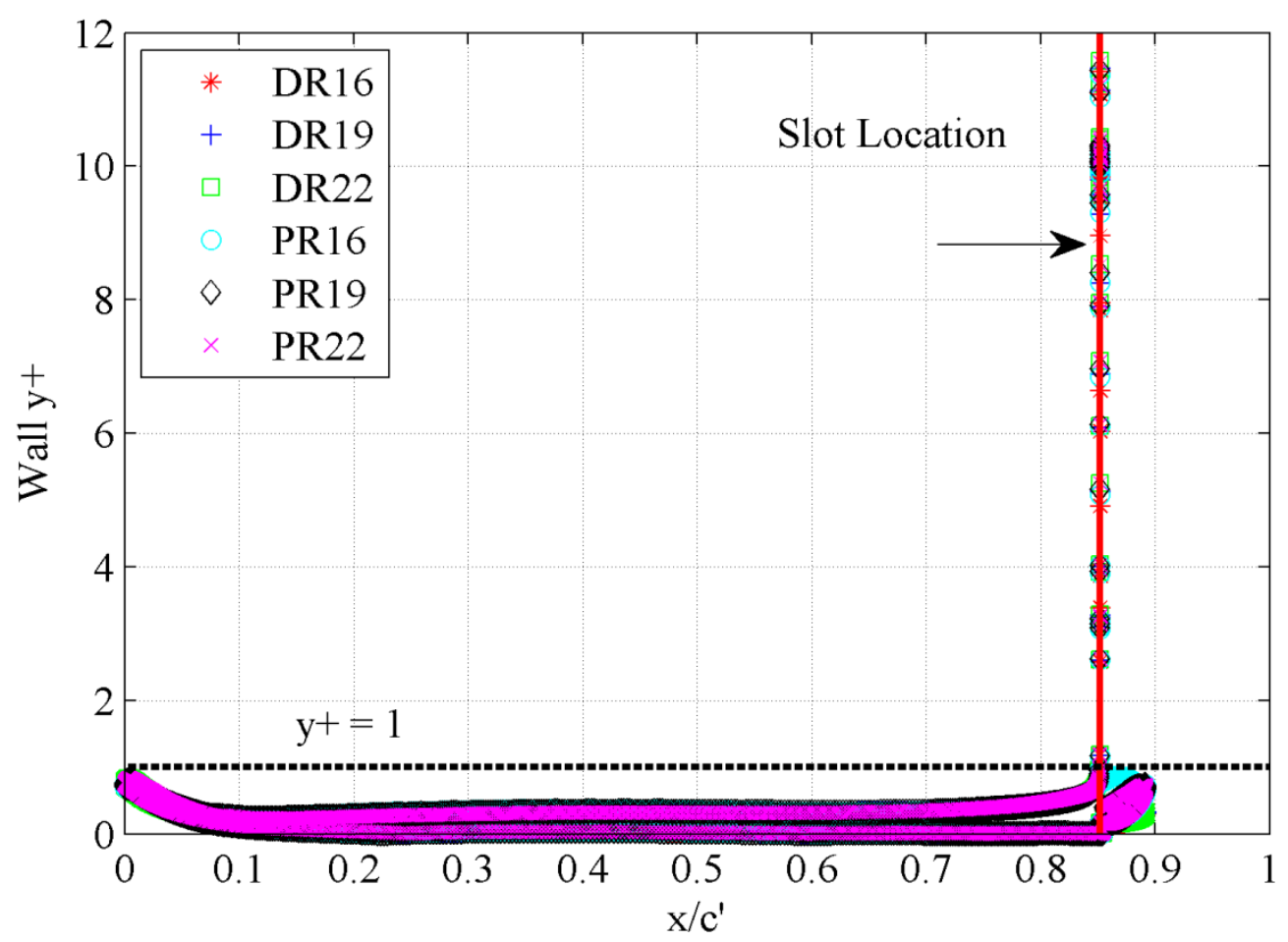

Figure A-9-7: $y^{+}$values of the airfoil/flap configurations, $\alpha=-5^{\circ}, 90^{\circ}$ flaps

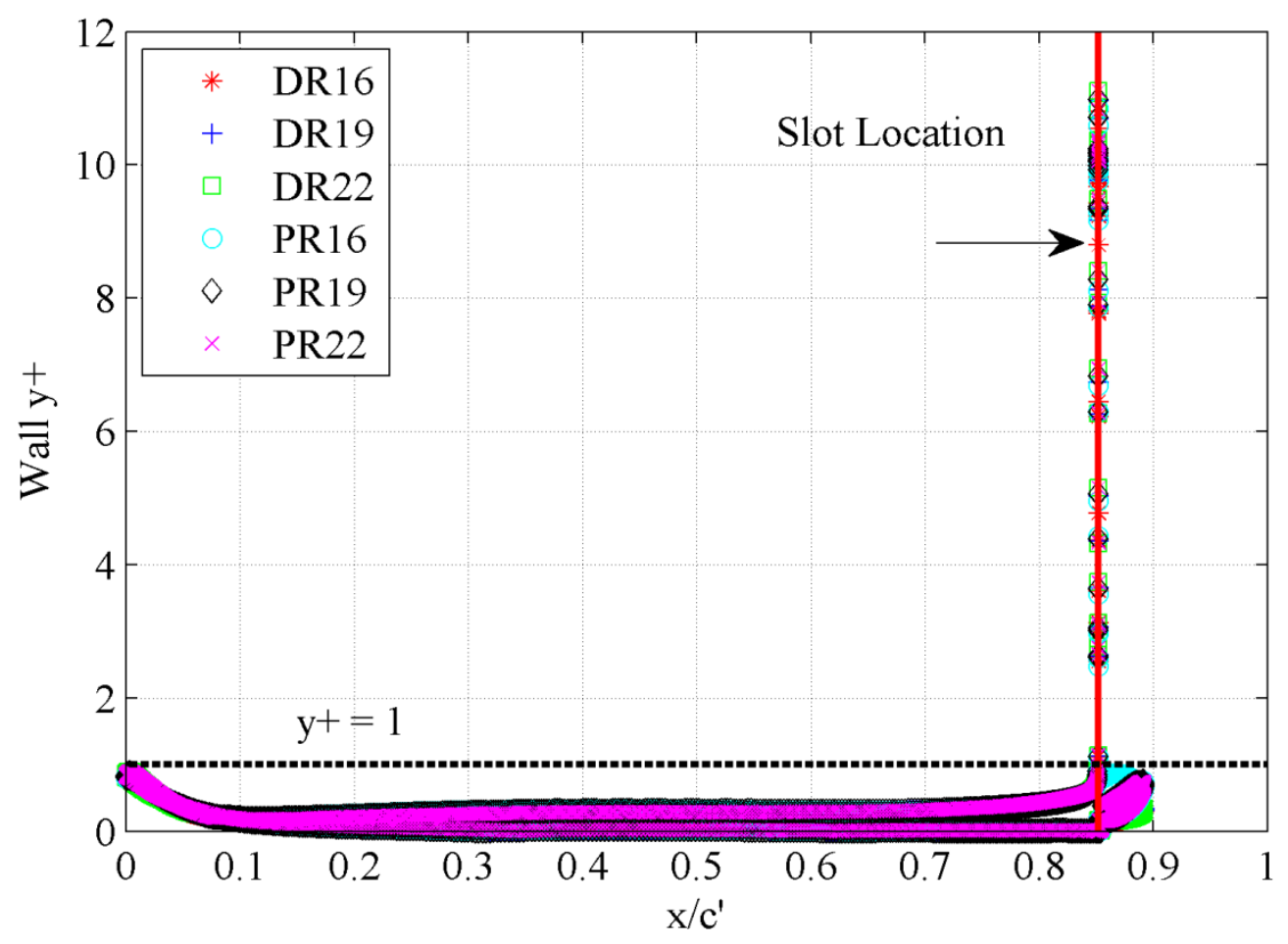

Figure A-9-8: $y^{+}$values of the airfoil/flap configurations, $\alpha=3^{\circ}, 90^{\circ}$ flaps 